\title{
Rock Cores in Monroe, Lawrence, and Surrounding Counties
}

\author{
Brian D. Keith and Todd A. Thompson \\ Indiana Geological and Water Survey, Indiana University, Bloomington, Indiana \\ E-mail: tthomps@indiana.edu \\ Received 01/01/2020 \\ Accepted for publication 01/01/2020 \\ Published 04/27/2020 \\ Suggested citation: Keith, B. D., and Thompson, T. A., 2020, Rock cores in Monroe, Lawrence, and surrounding Counties: Indiana \\ Geological and Water Survey, Indiana Journal of Earth Sciences, v. 2. DOI 10.14434/ijes.v2i1.27664
}

\begin{abstract}
Ninety new rock cores were collected to support geological mapping in Monroe and Lawrence Counties. These cores were systematically described using a coding system presented in A Corebook of Carbonate Rocks in Indiana (Thompson and Keith, 2015). Data from these cores were entered in the Indiana Geological and Water Survey Petroleum Database Management System and archived in the Survey core repository. This report summarizes the data collected and presents graphical descriptions of each core.
\end{abstract}

\section{INTRODUCTION}

As part of the federally funded STATEMAP portion of the National Cooperative Geologic Mapping Program, bedrock geological mapping was conducted in Monroe and Lawrence Counties from 2004 to 2014. This mapping resulted in a series of Indiana Geological Survey (now Indiana Geological and Water Survey [IGWS]) preliminary maps (Keith and others, 2008, 2009b, 2010, 2011, 2012, 2013; Thompson and others, 2008, 2009, 2010, 2011, 2012, 2013), several miscellaneous maps (Thompson and others, 2007; Hasenmueller and others, 2008, 2011; Keith and others, 2009a, 2014a), and a digital compilation (Keith and others, 2014b). Besides measuring hundreds of outcrops, we collected 90 rock cores. This brief report summarizes the collected data and presents graphical descriptions of those cores.

\section{METHODS}

Rock cores were drilled to a depth of about $200 \mathrm{ft}$ or less throughout Monroe and Lawrence Counties with additional cores located in surrounding counties (fig. 1). Cores were typically collected at high topographic areas and placed to retrieve important stratigraphic horizons not present in nearby outcrops. In Lawrence County, we strategically placed the cores to produce down-dip cross sections. All cores were sawed in half and described in detail at the Indiana Geological and Water Survey's Material Testing Facility.

After wetting, the cores were described using the coding system in Thompson and Keith (2015), the descriptive data stored, and stratigraphic columns created using the Windows ${ }^{\text {TII }}$.NET program Column (v. 1.02). To rapidly describe the thousands of units from the cores, we did 


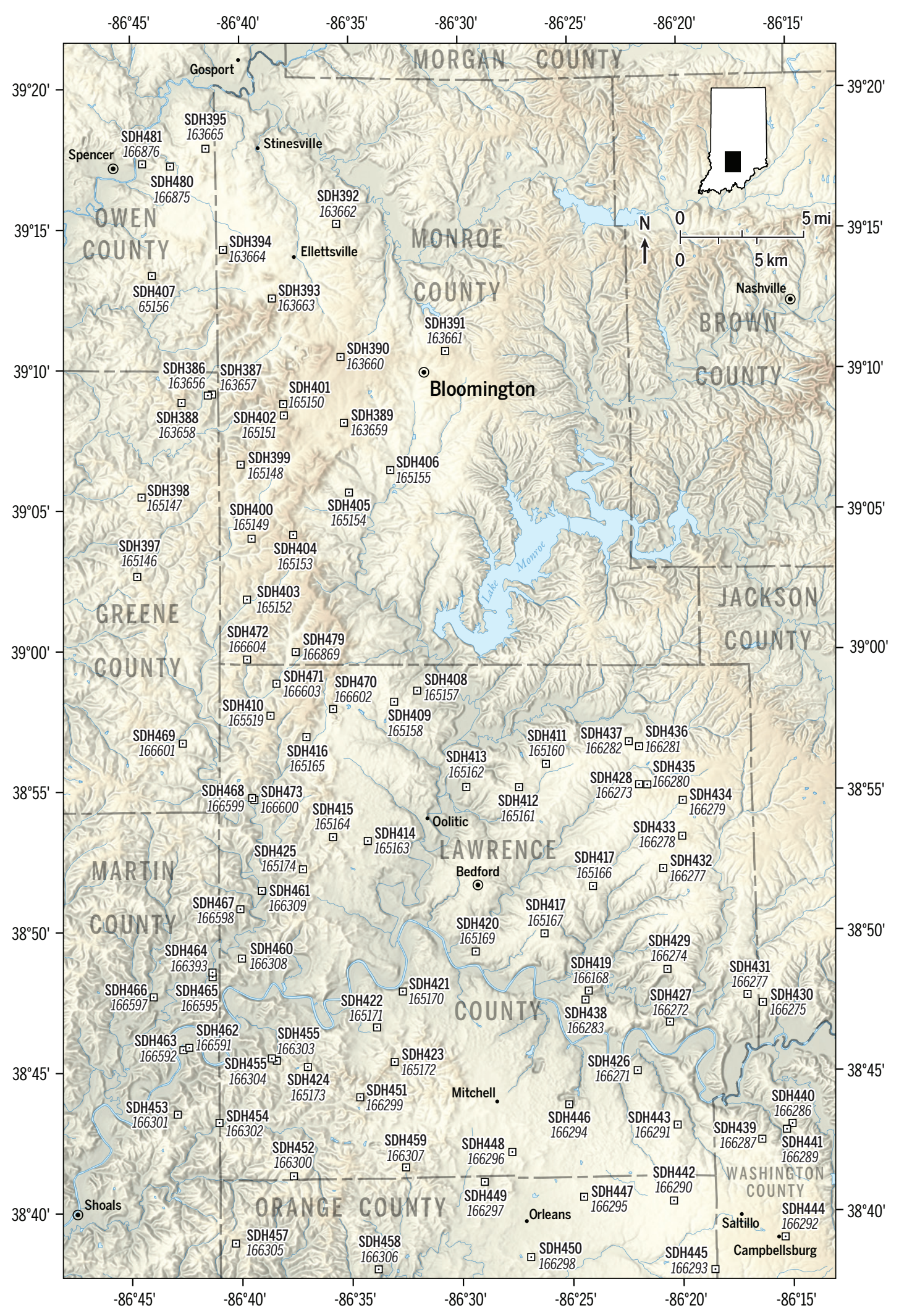

Figure 1. Hillshade map of south-central Indiana showing the locations of cores. Note that cores are labeled on map below, in Table 1, and in Appendix A as SDHxxx rather than SDH xxx (with a space). The the second number is used in the Petroleum Database Management System. 
not use Munsell color charts to identify colors. We based the contacts between stratigraphic horizons on a review of the literature. We then refined these as we described the cores in greater detail than is possible in outcrop. Locations and stratigraphic data were entered into the IGWS Petroleum Data Management System (PDMS) (https://igws.indiana.edu/pdms/). The cores were accessioned into the IGWS core repository.

\section{RESULTS}

Ninety cores having a total length of 2.61 miles were collected and described. Individual core lengths ranged from $20.3 \mathrm{ft}$ to $214.6 \mathrm{ft}$, with an average length of $153.3 \mathrm{ft}$ (Table 1) (Appendix A). Recovery of core was variable and influenced by the properties of the stratigraphic units. For instance, some sandstones were

Table 1. Core location and stratigraphic data.

\begin{tabular}{|c|c|c|c|c|c|c|c|}
\hline $\begin{array}{c}\text { Name } \\
\text { SDH* - site }^{*}\end{array}$ & $\begin{array}{l}\text { PDMS } \\
\text { Number }\end{array}$ & $\begin{array}{c}\text { UTM } \\
\text { Northing }\end{array}$ & $\begin{array}{l}\text { UTM } \\
\text { Easting }\end{array}$ & $\begin{array}{l}\text { Core Top } \\
\text { (ft) }\end{array}$ & \begin{tabular}{|c|} 
Core \\
Bottom $(\mathrm{ft})$
\end{tabular} & $\begin{array}{c}\text { Stratigraphic Unit } \\
\text { Top }\end{array}$ & $\begin{array}{c}\text { Stratigraphic Unit } \\
\text { Base }\end{array}$ \\
\hline $\begin{array}{c}\text { SDH386 } \\
\text { Liberty Church }\end{array}$ & 163656 & 4333199.5 & 524974.9 & 31.80 & 199.40 & $\begin{array}{c}\text { Stephensport } \\
\text { Group, Beech } \\
\text { Creek Limestone }\end{array}$ & $\begin{array}{l}\text { Blue River Group, } \\
\text { Ste. Genevieve } \\
\text { Limestone }\end{array}$ \\
\hline $\begin{array}{l}\text { SDH387 } \\
\text { Junction } \\
\text { Limestone 1 }\end{array}$ & 163657 & 4333754.0 & 526980.5 & 20.00 & 200.00 & $\begin{array}{l}\text { West Baden Group, } \\
\text { Elwren Formation }\end{array}$ & $\begin{array}{l}\text { Blue River Group, } \\
\text { Ste. Genevieve } \\
\text { Limestone }\end{array}$ \\
\hline $\begin{array}{l}\text { SDH388 } \\
\text { Junction } \\
\text { Limestone 2 }\end{array}$ & 163658 & 4333684.0 & 526699.0 & 2.70 & 200.00 & $\begin{array}{l}\text { Blue River Group, } \\
\text { Ste. Genevieve } \\
\text { Limestone }\end{array}$ & $\begin{array}{l}\text { Sanders Group, } \\
\text { Salem Limestone }\end{array}$ \\
\hline $\begin{array}{c}\text { SDH389 } \\
\text { Van Buren } \\
\text { Water Tower }\end{array}$ & 163659 & 4331891.7 & 535652.7 & 12.20 & 150.00 & $\begin{array}{l}\text { Blue River Group, } \\
\text { Paoli Limestone }\end{array}$ & $\begin{array}{l}\text { Blue River Group, } \\
\text { St. Louis Limestone }\end{array}$ \\
\hline $\begin{array}{c}\text { SDH390 } \\
\text { Loesch Road } \\
\text { Water Tower }\end{array}$ & 163660 & 4336223.9 & 535428.8 & 20.30 & 150.00 & $\begin{array}{l}\text { West Baden Group, } \\
\text { Bethel Formation }\end{array}$ & $\begin{array}{l}\text { Blue River Group, } \\
\text { St. Louis Limestone }\end{array}$ \\
\hline $\begin{array}{c}\text { SDH391 } \\
\text { IU Bell Tower }\end{array}$ & 163661 & 4336616.6 & 542306.6 & 20.80 & 200.00 & $\begin{array}{l}\text { Blue River Group, } \\
\text { St. Louis Limestone }\end{array}$ & $\begin{array}{l}\text { Borden Group, } \\
\text { Edwardsville } \\
\text { Formation }\end{array}$ \\
\hline $\begin{array}{c}\text { SDH392 } \\
\text { Peden Farm }\end{array}$ & 163662 & 4344988.4 & 535139.5 & 29.70 & 199.80 & $\begin{array}{l}\text { Blue River Group, } \\
\text { St. Louis Limestone }\end{array}$ & $\begin{array}{l}\text { Sanders Group, } \\
\text { Ramp Creek } \\
\text { Formation }\end{array}$ \\
\hline $\begin{array}{c}\text { SDH393 } \\
\text { Hoosier Energy } \\
\text { Microwave Tower }\end{array}$ & 163663 & 4340074.6 & 530914.4 & 22.50 & 200.00 & $\begin{array}{l}\text { West Baden Group, } \\
\text { Sample Formation }\end{array}$ & $\begin{array}{l}\text { Blue River Group, } \\
\text { St. Louis Limestone }\end{array}$ \\
\hline $\begin{array}{c}\text { SDH394 } \\
\text { Reeves Road } \\
\text { Water Tower }\end{array}$ & 163664 & 4343275.3 & 527696.7 & 24.00 & 200.00 & $\begin{array}{l}\text { West Baden Group, } \\
\text { Sample Formation }\end{array}$ & $\begin{array}{l}\text { Blue River Group, } \\
\text { St. Louis Limestone }\end{array}$ \\
\hline $\begin{array}{c}\text { SDH395 } \\
\text { Rolling Meadows } \\
\text { Golf Course }\end{array}$ & 163665 & 4349926.0 & 526544.0 & 10.00 & 210.05 & $\begin{array}{l}\text { Blue River Group, } \\
\text { Ste. Genevieve } \\
\text { Limestone }\end{array}$ & $\begin{array}{l}\text { Sanders Group, } \\
\text { Salem Limestone }\end{array}$ \\
\hline $\begin{array}{c}\text { SDH397 } \\
\text { Greene County } \\
\text { Middle School }\end{array}$ & 165146 & 4321746.6 & 522060.7 & 32.50 & 199.50 & $\begin{array}{l}\text { Stephensport } \\
\text { Group, Haney } \\
\text { Limestone }\end{array}$ & $\begin{array}{c}\text { West Baden Group, } \\
\text { Beaver Bend } \\
\text { Limestone }\end{array}$ \\
\hline $\begin{array}{c}\text { SDH398 } \\
\text { Solsberry } \\
\text { Water Tower }\end{array}$ & 165147 & 4326968.9 & 522343.1 & 10.00 & 60.00 & $\begin{array}{l}\text { Stephensport } \\
\text { Group, Big Clifty } \\
\text { Formation }\end{array}$ & $\begin{array}{c}\text { Stephensport } \\
\text { Group, Beech } \\
\text { Creek Limestone }\end{array}$ \\
\hline
\end{tabular}

*Survey drill hole 
Table 1 (continued). Core location and stratigraphic data

\begin{tabular}{|c|c|c|c|c|c|c|c|}
\hline $\begin{array}{c}\text { Name } \\
\text { SDH* - site }^{2}\end{array}$ & $\begin{array}{l}\text { PDMS } \\
\text { Number }\end{array}$ & $\begin{array}{c}\text { UTM } \\
\text { Northing } \\
\end{array}$ & $\begin{array}{c}\text { UTM } \\
\text { Easting }\end{array}$ & $\begin{array}{c}\text { Core Top } \\
\text { (ft) }\end{array}$ & \begin{tabular}{|c|} 
Core \\
Bottom $(\mathrm{ft})$ \\
\end{tabular} & $\begin{array}{c}\text { Stratigraphic Unit } \\
\text { Top }\end{array}$ & $\begin{array}{c}\text { Stratigraphic Unit } \\
\text { Base }\end{array}$ \\
\hline $\begin{array}{c}\text { SDH399 } \\
\text { Caldwell - Elwren }\end{array}$ & 165148 & 4329139.8 & 528858.3 & 14.00 & 200.00 & $\begin{array}{l}\text { West Baden Group, } \\
\text { Sample Formation }\end{array}$ & $\begin{array}{l}\text { Blue River Group, } \\
\text { Ste. Genevieve } \\
\text { Limestone }\end{array}$ \\
\hline $\begin{array}{l}\text { SDH400 } \\
\text { Doug Dixon }\end{array}$ & 165149 & 4324266.9 & 529578.8 & 12.00 & 170.00 & $\begin{array}{l}\text { Raccoon Creek } \\
\text { Group, Mansfield } \\
\text { Formation }\end{array}$ & $\begin{array}{l}\text { Blue River Group, } \\
\text { Ste. Genevieve } \\
\text { Limestone }\end{array}$ \\
\hline $\begin{array}{c}\text { SDH401 } \\
\text { Funkhouser } 1\end{array}$ & 165150 & 4333121.8 & 531661.7 & 10.00 & 200.00 & $\begin{array}{l}\text { West Baden Group, } \\
\text { Sample Formation }\end{array}$ & $\begin{array}{l}\text { Blue River Group, } \\
\text { St. Louis Limestone } \\
\end{array}$ \\
\hline $\begin{array}{c}\text { SDH402 } \\
\text { Funkhouser } 2\end{array}$ & 165151 & 4332374.9 & 531700.9 & 17.50 & 230.00 & $\begin{array}{l}\text { Blue River Group, } \\
\text { Paoli Limestone }\end{array}$ & $\begin{array}{l}\text { Blue River Group, } \\
\text { St. Louis Limestone }\end{array}$ \\
\hline $\begin{array}{c}\text { SDH403 } \\
\text { Hoosier Energy } \\
\text { Substation }\end{array}$ & 165152 & 4320255.3 & 529279.3 & 20.00 & 200.00 & $\begin{array}{l}\text { Blue River Group, } \\
\text { Ste. Genevieve } \\
\text { Limestone }\end{array}$ & $\begin{array}{l}\text { Blue River Group, } \\
\text { St. Louis Limestone }\end{array}$ \\
\hline $\begin{array}{c}\text { SDH404 } \\
\text { Van Buren Water } \\
\text { Tower - Mt. Zion }\end{array}$ & 165153 & 4324511.4 & 532318.8 & 18.50 & 200.00 & $\begin{array}{l}\text { Raccoon Creek } \\
\text { Group, Mansfield } \\
\text { Formation }\end{array}$ & $\begin{array}{l}\text { Blue River Group, } \\
\text { Ste. Genevieve } \\
\text { Limestone }\end{array}$ \\
\hline $\begin{array}{c}\text { SDH405 } \\
\text { Independent } \\
\text { Limestone Quarry }\end{array}$ & 165154 & 4327301.8 & 535986.1 & 0.00 & 170.00 & $\begin{array}{l}\text { Sanders Group, } \\
\text { Salem Limestone }\end{array}$ & $\begin{array}{l}\text { Borden Group, } \\
\text { Edwardsville } \\
\text { Formation }\end{array}$ \\
\hline $\begin{array}{l}\text { SDH406 } \\
\text { Sunoco Gas } \\
\text { Station }\end{array}$ & 165155 & 4328777.6 & 538704.5 & 4.50 & 150.00 & $\begin{array}{l}\text { Sanders Group, } \\
\text { Salem Limestone }\end{array}$ & $\begin{array}{l}\text { Borden Group, } \\
\text { Edwardsville } \\
\text { Formation }\end{array}$ \\
\hline $\begin{array}{l}\text { SDH407 } \\
\text { McCraken }\end{array}$ & 165156 & 4341550.7 & 523020.2 & 23.90 & 200.00 & $\begin{array}{l}\text { West Baden Group, } \\
\text { Sample Formation }\end{array}$ & $\begin{array}{c}\text { Blue River Group, } \\
\text { Ste. Genevieve } \\
\text { Limestone } \\
\end{array}$ \\
\hline $\begin{array}{c}\text { SDH408 } \\
\text { Guthrie Quarry }\end{array}$ & 165157 & 4314270.0 & 540472.7 & 6.80 & 191.00 & $\begin{array}{l}\text { Blue River Group, } \\
\text { St. Louis Limestone }\end{array}$ & $\begin{array}{l}\text { Borden Group, } \\
\text { Edwardsville } \\
\text { Formation }\end{array}$ \\
\hline $\begin{array}{c}\text { SDH409 } \\
\text { MOCO Gas Station }\end{array}$ & 165158 & 4313536.8 & 538957.1 & 24.20 & 201.40 & $\begin{array}{c}\text { Blue River Group, } \\
\text { St. Louis Limestone }\end{array}$ & $\begin{array}{l}\text { Sanders Group, } \\
\text { Salem Limestone }\end{array}$ \\
\hline $\begin{array}{c}\text { SDH410 } \\
\text { Siebolt Quarry } \\
\text { Floor }\end{array}$ & 165519 & 4313057.4 & 534943.2 & 5.90 & 201.00 & $\begin{array}{l}\text { Blue River Group, } \\
\text { Ste. Genevieve } \\
\text { Limestone }\end{array}$ & $\begin{array}{l}\text { Sanders Group, } \\
\text { Salem Limestone }\end{array}$ \\
\hline $\begin{array}{l}\text { SDH411 } \\
\text { Lazy Diamond } \\
\text { Farm Supply }\end{array}$ & 165160 & 4309450.5 & 548938.6 & 7.00 & 151.00 & $\begin{array}{l}\text { Sanders Group, } \\
\text { Salem Limestone }\end{array}$ & $\begin{array}{l}\text { Borden Group, } \\
\text { Edwardsville } \\
\text { Formation }\end{array}$ \\
\hline $\begin{array}{c}\text { SDH412 } \\
\text { Stevens Farm }\end{array}$ & 165161 & 4307917.4 & 547172.9 & 5.60 & 171.00 & $\begin{array}{l}\text { Sanders Group, } \\
\text { Salem Limestone }\end{array}$ & $\begin{array}{l}\text { Borden Group, } \\
\text { Edwardsville } \\
\text { Formation }\end{array}$ \\
\hline $\begin{array}{c}\text { SDH413 } \\
\text { Victor Oolitic } \\
\text { Quarry, abandoned }\end{array}$ & 165162 & 4307927.3 & 543705.9 & 3.00 & 161.00 & $\begin{array}{l}\text { Sanders Group, } \\
\text { Salem Limestone }\end{array}$ & $\begin{array}{l}\text { Borden Group, } \\
\text { Edwardsville } \\
\text { Formation }\end{array}$ \\
\hline $\begin{array}{c}\text { SDH414 } \\
\text { Feldon-Purdue } \\
\text { Agriculture Center }\end{array}$ & 165163 & 4304372.5 & 537222.4 & 5.40 & 131.00 & $\begin{array}{c}\text { Blue River Group, } \\
\text { Ste. Genevieve } \\
\text { Limestone }\end{array}$ & $\begin{array}{l}\text { Blue River Group, } \\
\text { St. Louis Limestone }\end{array}$ \\
\hline
\end{tabular}

*Survey drill hole 
Table 1 (continued). Core location and stratigraphic data

\begin{tabular}{|c|c|c|c|c|c|c|c|}
\hline $\begin{array}{c}\text { Name } \\
\text { SDH* - site }\end{array}$ & $\begin{array}{c}\text { PDMS } \\
\text { Number }\end{array}$ & $\begin{array}{c}\text { UTM } \\
\text { Northing }\end{array}$ & $\begin{array}{c}\text { UTM } \\
\text { Easting }\end{array}$ & $\begin{array}{l}\text { Core Top } \\
\text { (ft) }\end{array}$ & $\begin{array}{c}\text { Core } \\
\text { Bottom (ft) }\end{array}$ & $\begin{array}{c}\text { Stratigraphic Unit } \\
\text { Top }\end{array}$ & $\begin{array}{c}\text { Stratigraphic Unit } \\
\text { Base }\end{array}$ \\
\hline $\begin{array}{c}\text { SDH415 } \\
\text { Pritchett-Maul } \\
\text { Ridge Road }\end{array}$ & 165164 & 4304627.0 & 534937.7 & 29.00 & 165.10 & $\begin{array}{l}\text { West Baden Group, } \\
\text { Sample Formation }\end{array}$ & $\begin{array}{l}\text { Blue River Group, } \\
\text { Ste. Genevieve } \\
\text { Limestone }\end{array}$ \\
\hline $\begin{array}{c}\text { SDH416 } \\
\text { Oak Grove Church }\end{array}$ & 165165 & 4311209.9 & 533188.8 & 21.50 & 188.65 & $\begin{array}{l}\text { Blue River Group, } \\
\text { Paoli Limestone }\end{array}$ & $\begin{array}{l}\text { Blue River Group, } \\
\text { St. Louis Limestone }\end{array}$ \\
\hline $\begin{array}{c}\text { SDH417 } \\
\text { East Lawrence } \\
\text { Water Tower }\end{array}$ & 165166 & 4301410.5 & 552038.7 & 21.00 & 190.00 & $\begin{array}{l}\text { Blue River Group, } \\
\text { St. Louis Limestone }\end{array}$ & $\begin{array}{l}\text { Borden Group, } \\
\text { Edwardsville } \\
\text { Formation }\end{array}$ \\
\hline $\begin{array}{c}\text { SDH418 } \\
\text { Unity Missionary } \\
\text { Baptist Church }\end{array}$ & 165167 & 4298293.1 & 548848.7 & 37.90 & 208.55 & $\begin{array}{l}\text { Sanders Group, } \\
\text { Salem Limestone }\end{array}$ & $\begin{array}{l}\text { Borden Group, } \\
\text { Edwardsville } \\
\text { Formation }\end{array}$ \\
\hline $\begin{array}{c}\text { SDH419 } \\
\text { White River } \\
\text { Baptist Church }\end{array}$ & 165168 & 4293937.2 & 551543.4 & 33.00 & 200.00 & $\begin{array}{l}\text { Sanders Group, } \\
\text { Salem Limestone }\end{array}$ & $\begin{array}{l}\text { Borden Group, } \\
\text { Edwardsville } \\
\text { Formation }\end{array}$ \\
\hline $\begin{array}{c}\text { SDH420 } \\
\text { Ash Lake } \\
\text { Log Homes }\end{array}$ & 165169 & 4297097.2 & 544323.6 & 30.60 & 200.00 & $\begin{array}{l}\text { Blue River Group, } \\
\text { St. Louis Limestone }\end{array}$ & $\begin{array}{c}\text { Sanders Group, } \\
\text { Ramp Creek } \\
\text { Formation }\end{array}$ \\
\hline $\begin{array}{c}\text { SDH421 } \\
\text { Bluesprings } \\
\text { Caverns }\end{array}$ & 165170 & 4294465.4 & 539527.4 & 12.70 & 200.00 & $\begin{array}{l}\text { Blue River Group, } \\
\text { St. Louis Limestone }\end{array}$ & $\begin{array}{l}\text { Sanders Group, } \\
\text { Ramp Creek } \\
\text { Formation }\end{array}$ \\
\hline $\begin{array}{l}\text { SDH422 } \\
\text { Appleacres } \\
\text { Orchard } \\
\end{array}$ & 165171 & 4292100.4 & 537832.6 & 5.50 & 150.00 & $\begin{array}{c}\text { Blue River Group, } \\
\text { Ste. Genevieve } \\
\text { Limestone }\end{array}$ & $\begin{array}{l}\text { Blue River Group, } \\
\text { St. Louis Limestone }\end{array}$ \\
\hline $\begin{array}{l}\text { SDH423 } \\
\text { Bob Way }\end{array}$ & 165172 & 4289832.2 & 538995.2 & 30.00 & 200.00 & $\begin{array}{l}\text { Raccoon Creek } \\
\text { Group, Mansfield } \\
\text { Formation }\end{array}$ & $\begin{array}{l}\text { Blue River Group, } \\
\text { Ste. Genevieve } \\
\text { Limestone }\end{array}$ \\
\hline $\begin{array}{c}\text { SDH424 } \\
\text { Hoosier } \\
\text { National Forest }\end{array}$ & 165173 & 4289504.5 & 533280.8 & 15.50 & 190.00 & $\begin{array}{l}\text { Raccoon Creek } \\
\text { Group, Mansfield } \\
\text { Formation }\end{array}$ & $\begin{array}{l}\text { West Baden Group, } \\
\text { Bethel Formation }\end{array}$ \\
\hline $\begin{array}{l}\text { SDH425 } \\
\text { Ratkovich }\end{array}$ & 165174 & 4302508.0 & 532956.2 & 20.00 & 200.00 & $\begin{array}{l}\text { West Baden Group, } \\
\text { Elwren Formation }\end{array}$ & $\begin{array}{l}\text { Blue River Group, } \\
\text { St. Louis Limestone }\end{array}$ \\
\hline $\begin{array}{l}\text { SDH426 } \\
\text { Brumfield }\end{array}$ & 166271 & 4289293.2 & 554978.4 & 10.40 & 180.00 & $\begin{array}{l}\text { Blue River Group, } \\
\text { St. Louis Limestone }\end{array}$ & $\begin{array}{l}\text { Borden Group, } \\
\text { Edwardsville } \\
\text { Formation }\end{array}$ \\
\hline $\begin{array}{c}\text { SDH427 } \\
\text { Rosenbaum }\end{array}$ & 166272 & 4292488.0 & 557098.1 & 5.70 & 130.00 & $\begin{array}{l}\text { Sanders Group, } \\
\text { Salem Limestone }\end{array}$ & $\begin{array}{c}\text { Borden Group, } \\
\text { Edwardsville } \\
\text { Formation }\end{array}$ \\
\hline $\begin{array}{l}\text { SDH428 } \\
\text { Bill Todd } 1\end{array}$ & 166273 & 4308112.1 & 555068.1 & 8.10 & 80.00 & $\begin{array}{l}\text { Sanders Group, } \\
\text { Harrodsburg } \\
\text { Limestone }\end{array}$ & $\begin{array}{l}\text { Borden Group, } \\
\text { Edwardsville } \\
\text { Formation }\end{array}$ \\
\hline $\begin{array}{c}\text { SDH429 } \\
\text { Hidden Falls Camp }\end{array}$ & 166274 & 4295950.0 & 556935.4 & 14.50 & 120.00 & $\begin{array}{l}\text { Sanders Group, } \\
\text { Salem Limestone }\end{array}$ & $\begin{array}{l}\text { Borden Group, } \\
\text { Edwardsville } \\
\text { Formation }\end{array}$ \\
\hline $\begin{array}{l}\text { SDH430 } \\
\text { Larry McKeaigg }\end{array}$ & 166275 & 4293785.8 & 563211.4 & 4.00 & 150.00 & $\begin{array}{l}\text { Blue River Group, } \\
\text { St. Louis Limestone }\end{array}$ & $\begin{array}{l}\text { Sanders Group, } \\
\text { Ramp Creek } \\
\text { Formation }\end{array}$ \\
\hline
\end{tabular}

*Survey drill hole 
Table 1 (continued). Core location and stratigraphic data

\begin{tabular}{|c|c|c|c|c|c|c|c|}
\hline $\begin{array}{c}\text { Name } \\
\text { SDH* - site }^{*}\end{array}$ & $\begin{array}{l}\text { PDMS } \\
\text { Number }\end{array}$ & $\begin{array}{c}\text { UTM } \\
\text { Northing }\end{array}$ & $\begin{array}{c}\text { UTM } \\
\text { Easting }\end{array}$ & $\begin{array}{l}\text { Core Top } \\
\text { (ft) }\end{array}$ & \begin{tabular}{|c|} 
Core \\
Bottom (ft)
\end{tabular} & $\begin{array}{c}\text { Stratigraphic Unit } \\
\text { Top }\end{array}$ & $\begin{array}{c}\text { Stratigraphic Unit } \\
\text { Base }\end{array}$ \\
\hline $\begin{array}{l}\text { SDH431 } \\
\text { Greg Pickett }\end{array}$ & 166276 & 4294305.5 & 562202.8 & 14.70 & 110.00 & $\begin{array}{l}\text { Sanders Group, } \\
\text { Salem Limestone }\end{array}$ & $\begin{array}{l}\text { Borden Group, } \\
\text { Edwardsville } \\
\text { Formation }\end{array}$ \\
\hline $\begin{array}{c}\text { SDH432 } \\
\text { Wilma Jansing }\end{array}$ & 166277 & 4302593.1 & 556657.6 & 23.20 & 60.00 & $\begin{array}{l}\text { Sanders Group, } \\
\text { Ramp Creek } \\
\text { Formation }\end{array}$ & $\begin{array}{l}\text { Borden Group, } \\
\text { Edwardsville } \\
\text { Formation }\end{array}$ \\
\hline $\begin{array}{c}\text { SDH433 } \\
\text { Belcher - Erie } \\
\text { Church Road }\end{array}$ & 166278 & 4304724.3 & 557911.2 & 13.10 & 190.00 & $\begin{array}{l}\text { Blue River Group, } \\
\text { St. Louis Limestone }\end{array}$ & $\begin{array}{l}\text { Borden Group, } \\
\text { Edwardsville } \\
\text { Formation }\end{array}$ \\
\hline $\begin{array}{l}\text { SDH434 } \\
\text { Ratliff }\end{array}$ & 166279 & 4307082.7 & 557939.0 & 19.50 & 39.80 & $\begin{array}{l}\text { Sanders Group, } \\
\text { Ramp Creek } \\
\text { Formation }\end{array}$ & $\begin{array}{l}\text { Borden Group, } \\
\text { Edwardsville } \\
\text { Formation }\end{array}$ \\
\hline $\begin{array}{l}\text { SDH435 } \\
\text { Bill Todd } 2\end{array}$ & 166280 & 4308100.0 & 555609.4 & 24.70 & 200.00 & $\begin{array}{l}\text { Blue River Group, } \\
\text { St. Louis Limestone }\end{array}$ & $\begin{array}{l}\text { Sanders Group, } \\
\text { Ramp Creek } \\
\text { Formation }\end{array}$ \\
\hline $\begin{array}{c}\text { SDH436 } \\
\text { Blackwell } \\
\text { Limestone Co. } \\
\text { Quarry }\end{array}$ & 166281 & 4310607.8 & 555064.3 & 3.50 & 160.00 & $\begin{array}{l}\text { Sanders Group, } \\
\text { Salem Limestone }\end{array}$ & $\begin{array}{l}\text { Borden Group, } \\
\text { Edwardsville } \\
\text { Formation }\end{array}$ \\
\hline $\begin{array}{c}\text { SDH437 } \\
\text { Smith Sawmill }\end{array}$ & 166282 & 4310940.2 & 554388.7 & 17.50 & 80.00 & $\begin{array}{l}\text { Sanders Group, } \\
\text { Harrodsburg } \\
\text { Limestone }\end{array}$ & $\begin{array}{l}\text { Borden Group, } \\
\text { Edwardsville } \\
\text { Formation }\end{array}$ \\
\hline $\begin{array}{l}\text { SDH438 } \\
\text { Dalton }\end{array}$ & 166283 & 4294529.8 & 551755.8 & 4.50 & 40.00 & $\begin{array}{c}\text { Chert pebble } \\
\text { conglomerate, } \\
\text { unknown age }\end{array}$ & \\
\hline $\begin{array}{c}\text { SDH439 } \\
\text { Denny Brown }\end{array}$ & 166287 & 4285816.0 & 565165.0 & 6.70 & 80.00 & $\begin{array}{c}\text { Sanders Group, } \\
\text { Harrodsburg } \\
\text { Limestone }\end{array}$ & $\begin{array}{l}\text { Borden Group, } \\
\text { Edwardsville } \\
\text { Formation }\end{array}$ \\
\hline $\begin{array}{c}\text { SDH440 } \\
\text { Kevin Northway }\end{array}$ & 166286 & 4285435.1 & 564800.5 & 0.00 & 50.00 & $\begin{array}{l}\text { Sanders Group, } \\
\text { Ramp Creek } \\
\text { Formation }\end{array}$ & $\begin{array}{l}\text { Borden Group, } \\
\text { Edwardsville } \\
\text { Formation }\end{array}$ \\
\hline $\begin{array}{c}\text { SDH441 } \\
\text { Jennifer Cardwell }\end{array}$ & 166289 & 4284778.8 & 563175.5 & 12.20 & 180.00 & $\begin{array}{l}\text { Sanders Group, } \\
\text { Salem Limestone }\end{array}$ & $\begin{array}{l}\text { Borden Group, } \\
\text { Edwardsville } \\
\text { Formation }\end{array}$ \\
\hline $\begin{array}{c}\text { SDH442 } \\
\text { George Chastain }\end{array}$ & 166290 & 4280712.3 & 557364.9 & 28.80 & 201.10 & $\begin{array}{l}\text { Blue River Group, } \\
\text { St. Louis Limestone }\end{array}$ & $\begin{array}{l}\text { Sanders Group, } \\
\text { Harrodsburg } \\
\text { Limestone }\end{array}$ \\
\hline $\begin{array}{c}\text { SDH443 } \\
\text { Sugar Creek } \\
\text { Hunting Preserve }\end{array}$ & 166291 & 4285713.1 & 557607.3 & 35.60 & 210.00 & $\begin{array}{l}\text { Blue River Group, } \\
\text { St. Louis Limestone }\end{array}$ & $\begin{array}{l}\text { Sanders Group, } \\
\text { Ramp Creek } \\
\text { Formation }\end{array}$ \\
\hline $\begin{array}{c}\text { SDH444 } \\
\text { Town of } \\
\text { Campbellsburg }\end{array}$ & 166292 & 4278375.0 & 564254.7 & 56.00 & 80.00 & $\begin{array}{l}\text { Blue River Group, } \\
\text { St. Louis Limestone }\end{array}$ & $\begin{array}{l}\text { Blue River Group, } \\
\text { St. Louis Limestone }\end{array}$ \\
\hline $\begin{array}{c}\text { SDH445 } \\
\text { Steve Brown }\end{array}$ & 166293 & 4276202.9 & 560092.4 & 30.00 & 200.00 & $\begin{array}{l}\text { Blue River Group, } \\
\text { St. Louis Limestone }\end{array}$ & $\begin{array}{l}\text { Sanders Group, } \\
\text { Harrodsburg } \\
\text { Limestone }\end{array}$ \\
\hline
\end{tabular}

*Survey drill hole 
Table 1 (continued). Core location and stratigraphic data

\begin{tabular}{|c|c|c|c|c|c|c|c|}
\hline $\begin{array}{c}\text { Name } \\
\text { SDH* - site }^{*}\end{array}$ & $\begin{array}{l}\text { PDMS } \\
\text { Number }\end{array}$ & $\begin{array}{l}\text { UTM } \\
\text { Northing }\end{array}$ & $\begin{array}{c}\text { UTM } \\
\text { Easting }\end{array}$ & $\begin{array}{l}\text { Core Top } \\
\text { (ft) }\end{array}$ & $\begin{array}{c}\text { Core } \\
\text { Bottom (ft) }\end{array}$ & $\begin{array}{c}\text { Stratigraphic Unit } \\
\text { Top }\end{array}$ & $\begin{array}{c}\text { Stratigraphic Unit } \\
\text { Base }\end{array}$ \\
\hline $\begin{array}{l}\text { SDH446 } \\
\text { Spring Mill } \\
\text { State Park }\end{array}$ & 166294 & 4287046.5 & 550476.4 & 6.50 & 220.00 & $\begin{array}{l}\text { Blue River Group, } \\
\text { St. Louis Limestone }\end{array}$ & $\begin{array}{l}\text { Sanders Group, } \\
\text { Ramp Creek } \\
\text { Formation }\end{array}$ \\
\hline $\begin{array}{c}\text { SDH447 } \\
\text { Steve Ralston }\end{array}$ & 166295 & 4280957.5 & 551455.5 & 6.00 & 200.00 & $\begin{array}{l}\text { Blue River Group, } \\
\text { St. Louis Limestone }\end{array}$ & $\begin{array}{l}\text { Blue River Group, } \\
\text { St. Louis Limestone }\end{array}$ \\
\hline $\begin{array}{c}\text { SDH448 } \\
\text { Holiday Drive-In } \\
\text { Theater } \\
\end{array}$ & 166296 & 4283909.2 & 546732.8 & 3.50 & 200.00 & $\begin{array}{c}\text { Blue River Group, } \\
\text { Ste. Genevieve } \\
\text { Limestone }\end{array}$ & $\begin{array}{l}\text { Sanders Group, } \\
\text { Salem Limestone }\end{array}$ \\
\hline $\begin{array}{c}\text { SDH449 } \\
\text { Mark Taylor }\end{array}$ & 166297 & 4281941.1 & 544918.8 & 12.80 & 181.00 & $\begin{array}{l}\text { Blue River Group, } \\
\text { Paoli Limestone }\end{array}$ & $\begin{array}{l}\text { Blue River Group, } \\
\text { St. Louis Limestone }\end{array}$ \\
\hline $\begin{array}{c}\text { SDH450 } \\
\text { John Noblitt }\end{array}$ & 166298 & 4276990.4 & 547973.3 & 0.00 & 180.00 & $\begin{array}{l}\text { West Baden Group, } \\
\text { Bethel Formation }\end{array}$ & $\begin{array}{l}\text { Blue River Group, } \\
\text { St. Louis Limestone }\end{array}$ \\
\hline $\begin{array}{c}\text { SDH451 } \\
\text { Hoosier National } \\
\text { Forest - Georgia } \\
\text { Fire Tower }\end{array}$ & 166299 & 4287505.6 & 536730.4 & 8.10 & 200.00 & $\begin{array}{l}\text { Raccoon Creek } \\
\text { Group, Mansfield } \\
\text { Formation }\end{array}$ & $\begin{array}{c}\text { West Baden Group, } \\
\text { Beaver Bend } \\
\text { Limestone }\end{array}$ \\
\hline $\begin{array}{c}\text { SDH452 } \\
\text { Hoosier National } \\
\text { Forest - Brunner } \\
\text { Cemetery }\end{array}$ & 166300 & 4282306.1 & 532368.2 & 8.10 & 200.00 & $\begin{array}{l}\text { Raccoon Creek } \\
\text { Group, Mansfield } \\
\text { Formation }\end{array}$ & $\begin{array}{l}\text { West Baden Group, } \\
\text { Sample Formation }\end{array}$ \\
\hline $\begin{array}{c}\text { SDH453 } \\
\text { Robert White }\end{array}$ & 166301 & 4286361.0 & 524728.3 & 8.90 & 150.00 & $\begin{array}{c}\text { Raccoon Creek } \\
\text { Group, Mansfield } \\
\text { Formation }\end{array}$ & $\begin{array}{l}\text { West Baden Group, } \\
\text { Elwren Formation }\end{array}$ \\
\hline $\begin{array}{c}\text { SDH454 } \\
\text { Indian Creek Stone } \\
\text { Products Yard }\end{array}$ & 166302 & 4285815.0 & 527470.6 & 10.00 & 200.00 & $\begin{array}{c}\text { West Baden Group, } \\
\text { Beaver Bend } \\
\text { Limestone }\end{array}$ & $\begin{array}{l}\text { Blue River Group, } \\
\text { St. Louis Limestone }\end{array}$ \\
\hline $\begin{array}{c}\text { SDH455 } \\
\text { Indian Creek Stone } \\
\text { Products Farm }\end{array}$ & 166303 & 4289909.3 & 531241.0 & 6.00 & 120.00 & $\begin{array}{c}\text { Raccoon Creek } \\
\text { Group, Mansfield } \\
\text { Formation }\end{array}$ & $\begin{array}{c}\text { Stephensport } \\
\text { Group, Big Clifty } \\
\text { Formation }\end{array}$ \\
\hline $\begin{array}{c}\text { SDH456 } \\
\text { Indian Creek Stone } \\
\text { Products Quarry }\end{array}$ & 166304 & 4290062.8 & 530916.5 & 5.40 & 200.00 & $\begin{array}{c}\text { Stephensport } \\
\text { Group, Big Clifty } \\
\text { Formation }\end{array}$ & $\begin{array}{l}\text { Blue River Group, } \\
\text { Ste. Genevieve } \\
\text { Limestone }\end{array}$ \\
\hline $\begin{array}{c}\text { SDH457 } \\
\text { Hoosier National } \\
\text { Forest Turnaround } \\
\text { at Scarlet }\end{array}$ & 166305 & 4277877.3 & 528555.5 & 31.50 & 200.00 & $\begin{array}{l}\text { Raccoon Creek } \\
\text { Group, Mansfield } \\
\text { Formation }\end{array}$ & $\begin{array}{l}\text { Stephensport } \\
\text { Group, Big Clifty } \\
\text { Formation }\end{array}$ \\
\hline $\begin{array}{c}\text { SDH458 } \\
\text { Charles Johnson }\end{array}$ & 166306 & 4276181.4 & 537944.8 & 7.80 & 200.00 & $\begin{array}{l}\text { West Baden Group, } \\
\text { Elwren Formation }\end{array}$ & $\begin{array}{c}\text { Blue River Group, } \\
\text { Ste. Genevieve } \\
\text { Limestone }\end{array}$ \\
\hline $\begin{array}{l}\text { SDH459 } \\
\text { Watterson }\end{array}$ & 166307 & 4282893.6 & 539744.9 & 15.30 & 200.00 & $\begin{array}{c}\text { Raccoon Creek } \\
\text { Group, Mansfield } \\
\text { Formation }\end{array}$ & $\begin{array}{c}\text { Blue River Group, } \\
\text { Ste. Genevieve } \\
\text { Limestone }\end{array}$ \\
\hline $\begin{array}{l}\text { SDH460 } \\
\text { Dillman }\end{array}$ & 166308 & 4296635.1 & 528954.4 & 10.60 & 200.00 & $\begin{array}{l}\text { Raccoon Creek } \\
\text { Group, Mansfield } \\
\text { Formation }\end{array}$ & $\begin{array}{l}\text { Blue River Group, } \\
\text { Ste. Genevieve } \\
\text { Limestone }\end{array}$ \\
\hline $\begin{array}{c}\text { SDH461 } \\
\text { Bledsoe Quarry }\end{array}$ & 166309 & 4301093.6 & 530258.4 & 5.80 & 126.00 & $\begin{array}{c}\text { Blue River Group, } \\
\text { Ste. Genevieve } \\
\text { Limestone }\end{array}$ & $\begin{array}{l}\text { Blue River Group, } \\
\text { St. Louis Limestone }\end{array}$ \\
\hline
\end{tabular}

*Survey drill hole 
Table 1 (continued). Core location and stratigraphic data

\begin{tabular}{|c|c|c|c|c|c|c|c|}
\hline $\begin{array}{c}\text { Name } \\
\text { SDH }^{*} \text { - site }\end{array}$ & $\begin{array}{l}\text { PDMS } \\
\text { Number }\end{array}$ & $\begin{array}{c}\text { UTM } \\
\text { Northing }\end{array}$ & $\begin{array}{c}\text { UTM } \\
\text { Easting }\end{array}$ & $\begin{array}{c}\text { Core Top } \\
\text { (ft) }\end{array}$ & $\begin{array}{c}\text { Core } \\
\text { Bottom (ft) }\end{array}$ & $\begin{array}{c}\text { Stratigraphic Unit } \\
\text { Top }\end{array}$ & $\begin{array}{c}\text { Stratigraphic Unit } \\
\text { Base }\end{array}$ \\
\hline $\begin{array}{c}\text { SDH462 } \\
\text { Jim Williams } 2\end{array}$ & 166591 & 4290602.6 & 525098.8 & 20.10 & 120.00 & $\begin{array}{l}\text { Blue River Group, } \\
\text { Paoli Limestone }\end{array}$ & $\begin{array}{l}\text { Blue River Group, } \\
\text { Ste. Genevieve } \\
\text { Limestone }\end{array}$ \\
\hline $\begin{array}{c}\text { SDH463 } \\
\text { Jim Williams } 1\end{array}$ & 166592 & 4290762.7 & 525488.4 & 7.00 & 150.00 & $\begin{array}{l}\text { West Baden Group, } \\
\text { Elwren Formation }\end{array}$ & $\begin{array}{c}\text { Blue River Group, } \\
\text { Ste. Genevieve } \\
\text { Limestone }\end{array}$ \\
\hline $\begin{array}{c}\text { SDH464 } \\
\text { Indian Creek } \\
\text { Quarries } 1\end{array}$ & 166593 & 4295405.1 & 527018.0 & 0.00 & 180.00 & $\begin{array}{c}\text { Blue River Group, } \\
\text { Ste. Genevieve } \\
\text { Limestone }\end{array}$ & $\begin{array}{l}\text { Blue River Group, } \\
\text { St. Louis Limestone }\end{array}$ \\
\hline $\begin{array}{c}\text { SDH465 } \\
\text { Indian Creek } \\
\text { Quarries } 2\end{array}$ & 166595 & 4295711.3 & 527010.4 & 13.00 & 80.00 & $\begin{array}{l}\text { West Baden Group, } \\
\text { Bethel Formation }\end{array}$ & $\begin{array}{l}\text { Blue River Group, } \\
\text { Ste. Genevieve } \\
\text { Limestone }\end{array}$ \\
\hline $\begin{array}{c}\text { SDH466 } \\
\text { Mary Baker } 2\end{array}$ & 166597 & 4294081.9 & 523141.6 & 0.00 & 170.00 & $\begin{array}{l}\text { Raccoon Creek } \\
\text { Group, Mansfield } \\
\text { Formation }\end{array}$ & $\begin{array}{l}\text { Blue River Group, } \\
\text { Ste. Genevieve } \\
\text { Limestone }\end{array}$ \\
\hline $\begin{array}{l}\text { SDH467 } \\
\text { Brock }\end{array}$ & 166598 & 4299878.5 & 528845.2 & 10.00 & 200.00 & $\begin{array}{l}\text { West Baden Group, } \\
\text { Sample Formation }\end{array}$ & $\begin{array}{c}\text { Blue River Group, } \\
\text { Ste. Genevieve } \\
\text { Limestone }\end{array}$ \\
\hline $\begin{array}{l}\text { SDH468 } \\
\text { Heritage Quarry } 1\end{array}$ & 166599 & 4307079.0 & 529766.5 & 5.50 & 130.00 & $\begin{array}{c}\text { Blue River Group, } \\
\text { Ste. Genevieve } \\
\text { Limestone }\end{array}$ & $\begin{array}{l}\text { Blue River Group, } \\
\text { St. Louis Limestone }\end{array}$ \\
\hline $\begin{array}{l}\text { SDH469 } \\
\text { Padgett }\end{array}$ & 166601 & 4310779.0 & 525053.0 & 5.00 & 200.00 & $\begin{array}{l}\text { Raccoon Creek } \\
\text { Group, Mansfield } \\
\text { Formation }\end{array}$ & $\begin{array}{l}\text { Blue River Group, } \\
\text { Ste. Genevieve } \\
\text { Limestone }\end{array}$ \\
\hline $\begin{array}{c}\text { SDH470 } \\
\text { Lowder Cemetery }\end{array}$ & 166602 & 4312614.3 & 530825.7 & 5.00 & 200.00 & $\begin{array}{l}\text { Raccoon Creek } \\
\text { Group, Mansfield } \\
\text { Formation }\end{array}$ & $\begin{array}{l}\text { Blue River Group, } \\
\text { Ste. Genevieve } \\
\text { Limestone }\end{array}$ \\
\hline $\begin{array}{c}\text { SDH471 } \\
\text { Armstrong Quarry } \\
\text { Floor }\end{array}$ & 166603 & 4314722.9 & 531218.7 & 3.50 & 80.00 & $\begin{array}{l}\text { Blue River Group, } \\
\text { Ste. Genevieve } \\
\text { Limestone }\end{array}$ & $\begin{array}{l}\text { Blue River Group, } \\
\text { St. Louis Limestone }\end{array}$ \\
\hline $\begin{array}{c}\text { SDH472 } \\
\text { Roger Fancher }\end{array}$ & 166604 & 4316310.3 & 529287.0 & 0.00 & 200.00 & $\begin{array}{c}\text { Raccoon Creek } \\
\text { Group, Mansfield } \\
\text { Formation }\end{array}$ & $\begin{array}{l}\text { Blue River Group, } \\
\text { Ste. Genevieve } \\
\text { Limestone }\end{array}$ \\
\hline $\begin{array}{l}\text { SDH473 } \\
\text { Heritage Quarry } 2\end{array}$ & 166600 & 4307196.2 & 529621.4 & 9.10 & 60.00 & $\begin{array}{c}\text { Blue River Group, } \\
\text { Ste. Genevieve } \\
\text { Limestone }\end{array}$ & $\begin{array}{c}\text { Blue River Group, } \\
\text { Ste. Genevieve } \\
\text { Limestone }\end{array}$ \\
\hline $\begin{array}{c}\text { SDH479 } \\
\text { Linthicum United } \\
\text { Methodist } \\
\text { Cemetery }\end{array}$ & 166869 & 4316806.9 & 532479.5 & 15.00 & 170.00 & $\begin{array}{l}\text { West Baden Group, } \\
\text { Elwren Formation }\end{array}$ & $\begin{array}{l}\text { Blue River Group, } \\
\text { Ste. Genevieve } \\
\text { Limestone }\end{array}$ \\
\hline $\begin{array}{c}\text { SDH480 } \\
\text { McCormick's } \\
\text { Creek State Park }\end{array}$ & 166875 & 4348753.8 & 524212.3 & 27.20 & 210.00 & $\begin{array}{l}\text { Blue River Group, } \\
\text { Ste. Genevieve } \\
\text { Limestone }\end{array}$ & $\begin{array}{l}\text { Sanders Group, } \\
\text { Salem Limestone }\end{array}$ \\
\hline $\begin{array}{l}\text { SDH481 } \\
\text { Platt }\end{array}$ & 166876 & 4348903.1 & 522377.4 & 5.40 & 220.00 & $\begin{array}{l}\text { Blue River Group, } \\
\text { St. Louis Limestone }\end{array}$ & $\begin{array}{l}\text { Borden Group, } \\
\text { Edwardsville } \\
\text { Formation }\end{array}$ \\
\hline
\end{tabular}

*Survey drill hole 
weathered and highly friable, and some limestones had void spaces owing to dissolution. Despite these issues, total recovery for all cores was 97 percent, with the poorest recovery in SDH-444 at 59 percent. Thirty-nine cores had no losses whatsoever.

Rocks exposed in Monroe and Lawrence Counties span six stratigraphic groups (fig. 2), ranging from the Middle Mississippian Borden Group along the eastern edge of the mapping area to the Lower Pennsylvanian Raccoon Creek Group in the western part. This distribution of oldest to youngest rocks is related to the regional dip of approximately 30 to $35 \mathrm{ft}$ per mile to

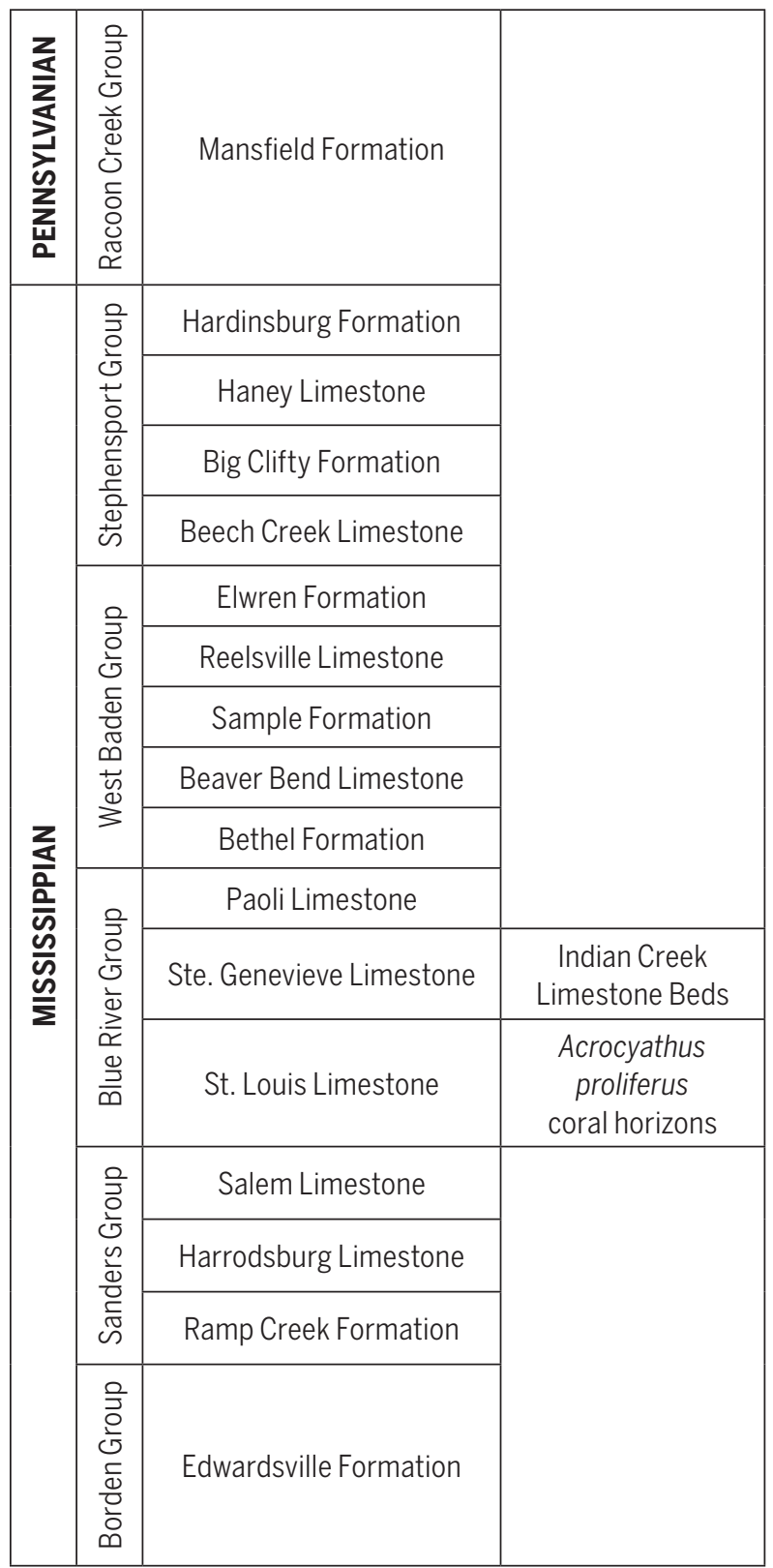

Figure 2. Stratigraphic column of the units and horizons highlighted in the cores. the southwest. This same stratigraphic range is present in the cores, encompassing 17 formations (fig. 3). The Blue River Group Ste. Genevieve Limestone and St. Louis Limestone were the most commonly cored units, primarily because they are thick formations encountered when drilling from higher stratigraphic units and are common hill formers in the mapped area. Also, we located core sites to retrieve rock from the middle of the St. Louis Limestone, where the coral Acrocyathus proliferus occurs in one or two beds throughout the two-county area. We found the coral horizons to be useful in correlating rock units within the St. Louis Limestone. When the two coral horizons are present in core, they are typically separated by 10 to $14 \mathrm{ft}$. Similarly, the Indian Creek Limestone Beds, defined by Connor (1986) but not formally adopted by the Indiana Geological and Water Survey, are useful for correlations within the Ste. Genevieve Limestone. Other formal and informal members and beds are not shown in the stratigraphic columns. The authors intend to define these units more clearly in a future publication.

Our intent in drilling the cores was to provide more stratigraphic contact data between formations than could be found in the relatively limited outcrops and quarry exposures in the map area. We described the cores in relative detail as efficiently as possible, given the volume of core material, the need for stratigraphic data for mapping, and the contractual deadlines for

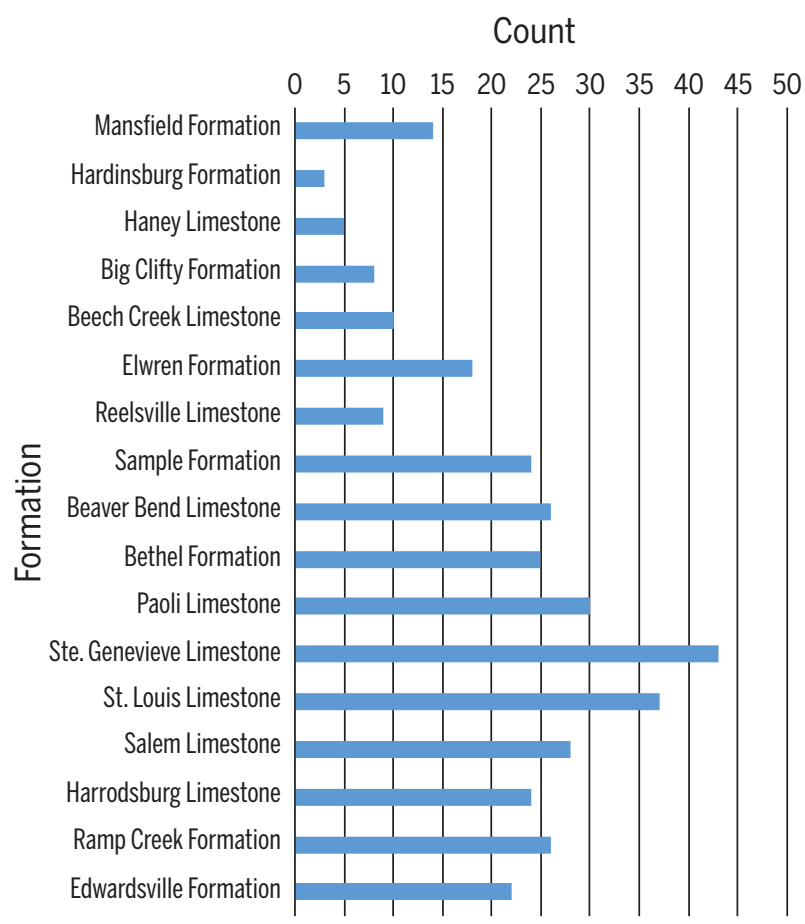

Figure 3. Histogram illustrating the number of cores that contain each formation. 
producing the maps. The cores do provide a wealth of information about the units that can be used for further research and interpretation beyond the mapping for which they were acquired. For example, the Acrocyathus coral and Indian Creek Limestone Beds may represent time horizons related to sea level change across the Illinois Basin.

\section{ACKNOWLEDGMENTS}

The authors thank the water companies, cell tower operators, quarries, mills, and landowners that permitted us to retrieve cores. Their enthusiastic support made the months of drilling enjoyable. All cores were collected by Geo-Drill of Lexington, Kentucky. The coring would not have been accomplished without the support of the U.S. Geological Survey through the STATEMAP portion of the National Cooperative Mapping Program under numerous agreements (04HQAG0033, 05HQAG0 031, 06HQAG0013, 07HQAG0069, 08HQAG0089, G09 AC00164, G10AC00221, G11AC20237, G12AC20119, G 13AC00176, and G14AC00141). The agreements require the following statement: "The views and conclusions contained in this document are those of the authors and should not be interpreted as necessarily representing the official policies, either expressed or implied, of the U.S. Government."

\section{REFERENCES}

Conner, G. A., 1986, Type section for Indian Creek Limestone Beds in the Ste. Genevieve Formation of south central Indiana: Proceedings of the Indiana Academy of Science, v. 95 , p. 307-311.

Hasenmueller, W. A., Estell, C. M., Keith, B. D., and Thompson T. A., 2008, Bedrock geologic map of Monroe County, Indiana: Indiana Geological Survey Miscellaneous Map 73, scale 1:48,000.

Hasenmueller, W. A., Estell, C. M., Thompson, T. A., and Keith, B. D., 2011. Geologic map of the Allens Creek 7.5-minute quadrangle, Indiana: Indiana Geological Survey Miscellaneous Map 83, scale 1:24,000.

Keith, B. D., Hasenmueller, W. A., Estell, C. M., and Thompson, T. A., 2009a, Geologic map of the Clear Creek 7.5-minute quadrangle, Indiana: Indiana Geological Survey Miscellaneous Map 76, scale 1:24,000.

Keith, B. D., Thompson, T. A., Estell, C. M., and Hasenmueller, W. A., 2009b, Preliminary bedrock geologic map of the Bedford West 7.5-minute quadrangle, Indiana: Indiana Geological Survey Open-File Study 09-11, scale 1:24,000.

Keith, B. D., Thompson, T. A., Estell, C. M., and Hasenmueller, W. A., 2010, Preliminary bedrock geologic map of the Norman 7.5-minute quadrangle, Indiana: Indiana Geological Survey Open-File Study 10-03, scale 1:24,000.
Keith, B. D., Thompson T. A., and Hasenmueller, W. A., 2011, Preliminary bedrock geologic map of the Mitchell 7.5-minute quadrangle, Indiana: Indiana Geological Survey Open-File Study 11-03, scale 1:24,000.

Keith, B. D., Thompson, T. A., Hasenmueller, W. A., and Estell, C. M., 2008, Preliminary bedrock geologic map of the Oolitic 7.5-minute quadrangle, Indiana: Indiana Geological Survey Open-File Study 08-01, scale 1:24,000.

Keith, B. D., Thompson, T. A., Rupp, R. F., and Hasenmueller, W. A., 2012, Preliminary bedrock geologic map of the Huron 7.5-minute quadrangle, Indiana: Indiana Geological Survey Open-File Study 12-05, scale 1:24,000.

Keith, B. D., Thompson, T. A., and Rupp, R. F., 2013, Preliminary bedrock geologic map of the Owensburg 7.5-minute quadrangle, Indiana: Indiana Geological Survey Open-File Study 13-02, scale 1:24,000.

Keith, B. D., Thompson, T. A., Herrmann, E. W., Rupp, R. F., and Hasenmueller, W. A., 2014a, Geologic map of Lawrence County, Indiana: Indiana Geological Survey Miscellaneous Map 90, scale 1:48,000.

Keith, B. D., Thompson, T. A., Herrmann, E. W., Rupp, R. F., Hasenmueller, W. A., and Johnson, M. R., 2014b, Geology of Lawrence County, Indiana (digital compilation) [ESRI geodatabase based on the NCGMP09 geodatabase model]: Indiana Geological Survey, unpublished IGWS digital data, scale $1: 48,000$.

Thompson, T. A., and Keith, B. D., 2015, Corebook of carbonate and associated rocks in Indiana: Indiana Geological Survey Occasional Paper 74, 149 p.

Thompson, T. A., Keith, B. D., Hasenmueller, W. A., and Estell, C. M., 2007, Geologic map of the Bloomington 7.5-minute quadrangle, Indiana: Indiana Geological Survey Miscellaneous Map 75, scale 1:24,000.

Thompson, T. A., Keith, B. D., Hasenmueller, W. A., and Estell, C. M., 2008, Preliminary bedrock geologic map of the Bartlettsville 7.5-minute quadrangle, Indiana: Indiana Geological Survey Open-File Study 08-02, scale 1:24,000.

Thompson, T. A., Keith, B. D., Estell, C. M., and Hasenmueller, W. A., 2009, Preliminary bedrock geologic map of the Bedford East 7.5-minute quadrangle, Indiana: Indiana Geological Survey Open-File Study 09-10, scale 1:24,000.

Thompson, T. A., Keith, B. D., Estell, C. M., and Hasenmueller, W. A., 2010, Preliminary bedrock geologic map of the Tunnelton 7.5-minute quadrangle, Indiana: Indiana Geological Survey Open-File Study 10-02, scale 1:24,000.

Thompson, T. A., Keith, B. D., and Hasenmueller, W. A., 2011, Preliminary bedrock geologic map of the Campbellsburg 7.5-minute quadrangle, Indiana: Indiana Geological Survey Open-File Study 11-02, scale 1:24,000.

Thompson, T. A., Keith, B. D., Rupp, R. F., and Hasenmueller, W. A., 2012, Preliminary bedrock geologic map of the Georgia 7.5-minute quadrangle, Indiana: Indiana Geological Survey Open-File Study 12-04, scale 1:24,000.

Thompson, T. A., Keith, B. D., and Rupp, R. F., 2013, Preliminary bedrock geologic map of the Williams 7.5-minute quadrangle, Indiana: Indiana Geological Survey Open-File Study 13-03, scale 1:24,000. 


\section{APPENDIX A}

\section{Stratigraphic columns}

\section{KEY}

IGWS Petroleum

Database Mangement

System Number

Date

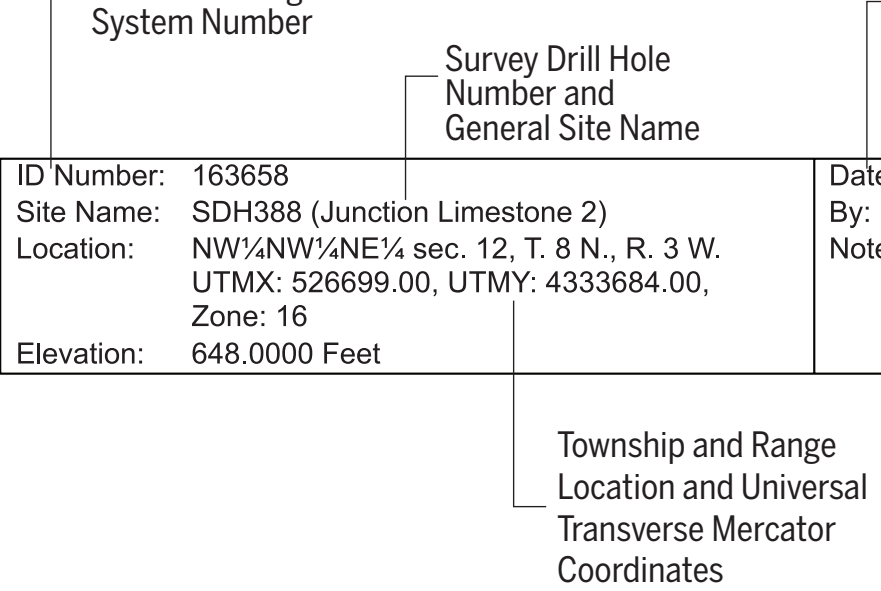

Described

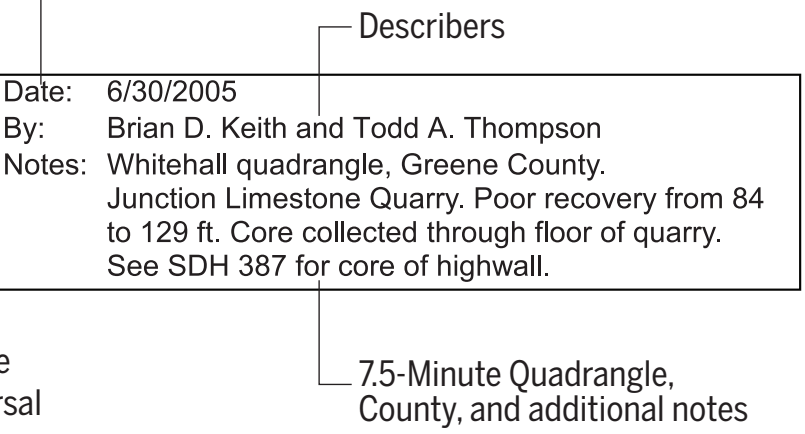

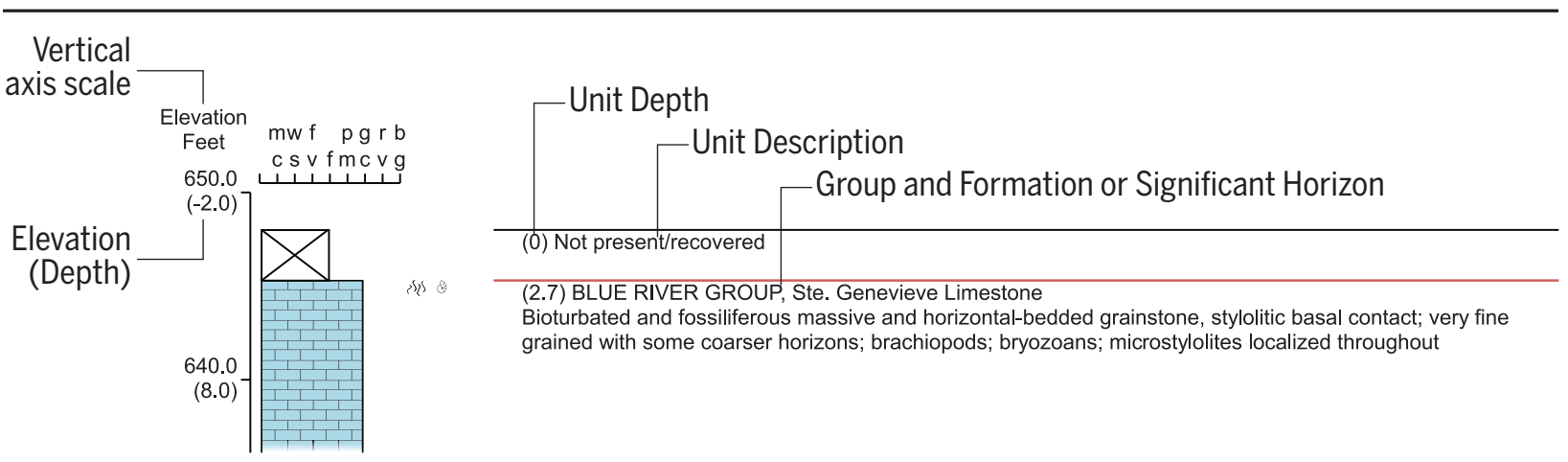




\section{KEY}

\section{DUNHAM CLASSIFICATION \\ (left to right) \\ m - mudstone \\ w - wackestone \\ $f$ - floatstone \\ p - packstone \\ g - grainstone \\ $r$ - rudstone \\ b - boundstone}

WENTWORTH

GRAIN SIZES

(left to right)

c - clay

s - silt

$v$ - very fine

$f$ - fine

$\mathrm{m}$ - medium

c - coarse

v - very coarse

g - gravel
LITHOLOGIES

$\because \because 9$ conglomerate

$\because:$ sandstone

$\because-\div$ siltstone

- shale

$\because \cdots$ mudstone

I1 limestone

7 dolostone

Co 5 limestone-clast breccia

B\&4 dolostone-clast breccia

- clay

$\Delta \Delta$ chert

스소 gypsum/anhydrite

coal
PRIMARY SEDIMENTARY STRUCTURE

cross bedding

产目 horizontal bedding

mipple bedding

드 flaser bedding

$\approx$ wavy bedding

as lenticular bedding

产学 rhythmic bedding

$\because \cdots$ interlaminated/interbedded

\section{GRAIN TYPE OR SECONDARY SEDIMENTARY FEATURE CONTACT}
(2) fossiliferous
$\odot \odot$ oolitic
- peloidal
$\oplus_{8}$ intraclast
๑) oncolitic
** birdseyes
¿3 mottled
$\mathrm{vvr}$ mudcracks
s)y bioturbated
disturbed bedding
사. rooted
§ slickensided

- sharp

mn irregular

--- gradational

凹

$\pi$ hardground 


\begin{tabular}{|c|c|c|}
\hline $\begin{array}{l}\text { I N Number: } \\
\text { Site Name: } \\
\text { Location: }\end{array}$ & 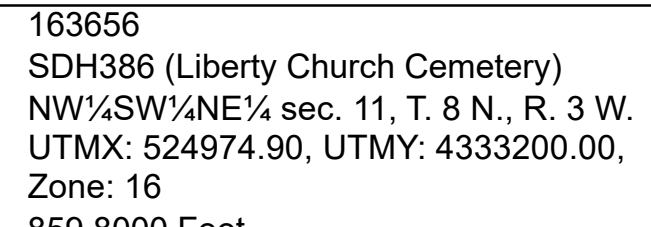 & $\begin{array}{ll}\text { Date: } & 7118 / 2005 \\
\text { By: } \\
\text { Notes: } \\
\text { Nodd A. Thompson and Brian D. Keith quadrangle, Greene County }\end{array}$ \\
\hline
\end{tabular}

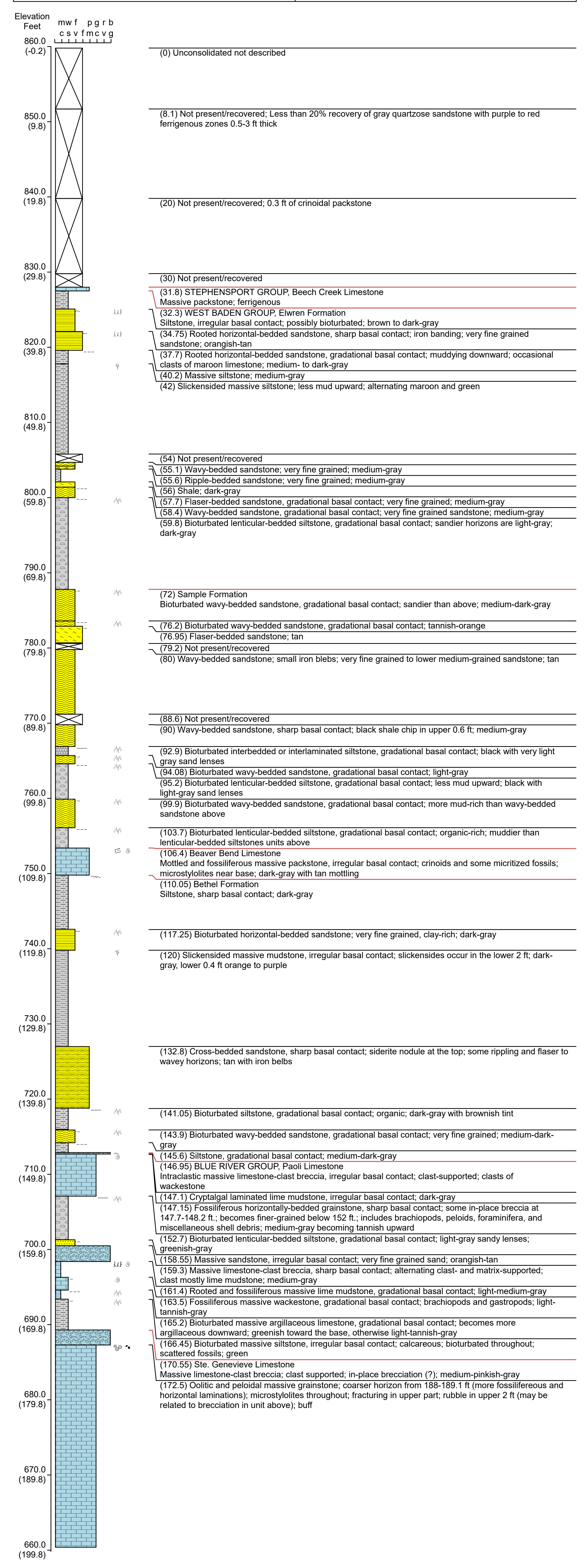

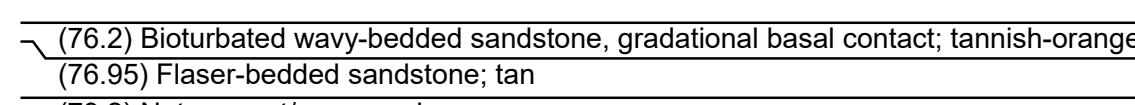

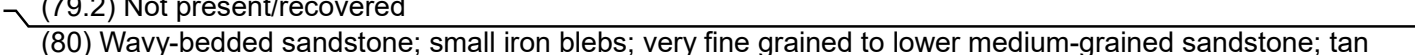

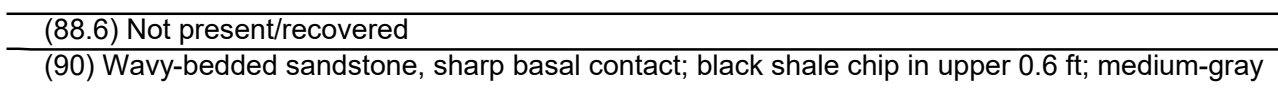

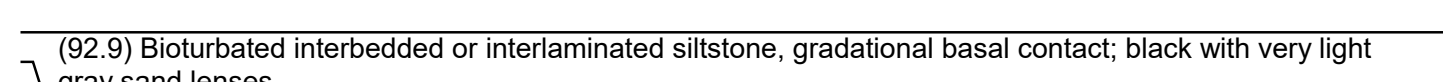

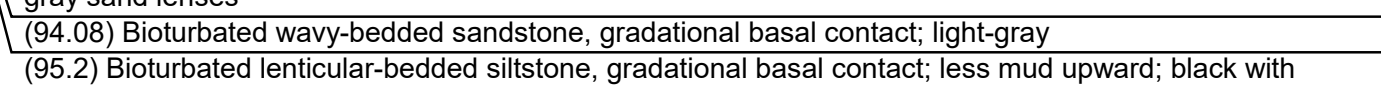

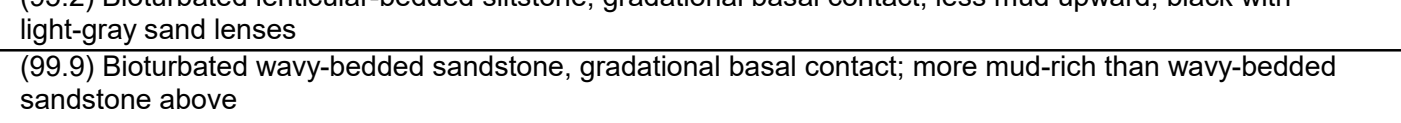

(117.25) Bioturbated horizontal-bedded sandstone; very fine grained, clay-rich; dark-gray

(132.8) Cross-bedded sandstone, sharp basal contact, siderite nodule at the top; some rippling and flaser to
wavey horizons; tan with iron helbs

(190.:

(141.05) Bioturbated siltstone, gradational basal contact; organic; dark-gray with brownish tint (143.9) Bioturbated wavy-bedded sandstone, gradational basal contact, very fine grained; medium-dark-

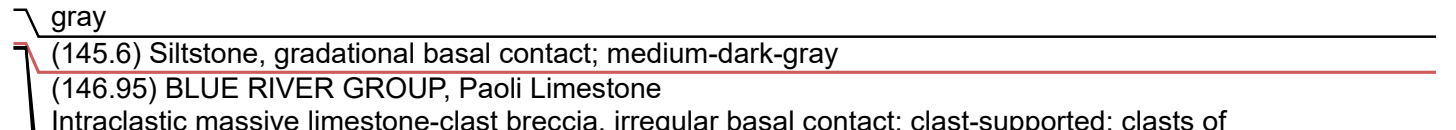

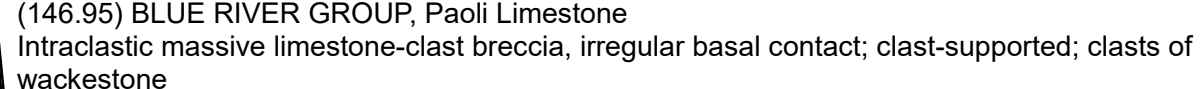

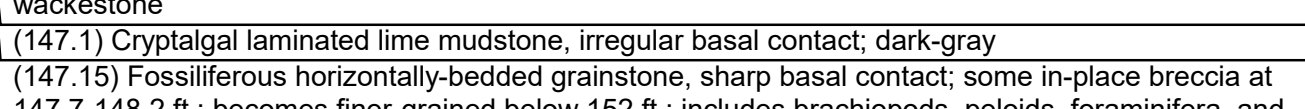

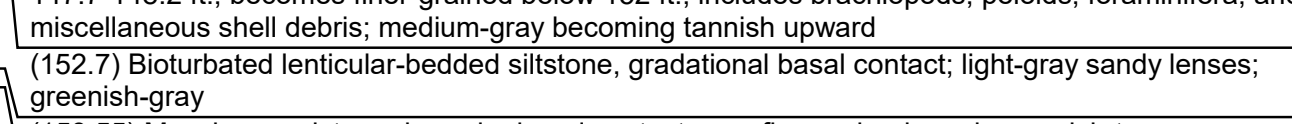
greenish-gray
(155.55) Massivive sandstone, irregular basal contact, very fine erained sand; ;rangish-tan
(159.3) Massive limestone-clast breccia, sharp basal contact; alternating clast- and matrix-supported; 每last mostly lime mudstone, medium-gray

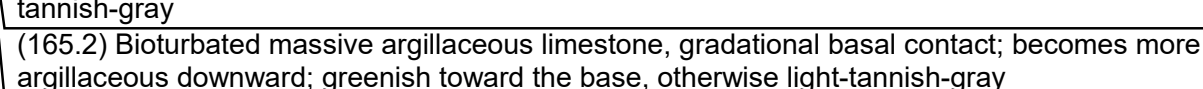

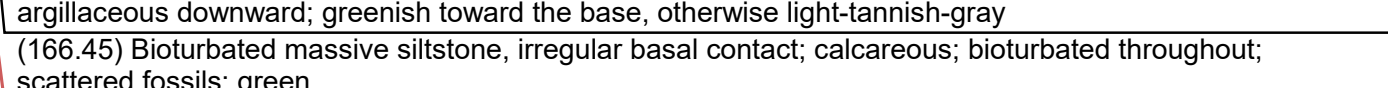
scattered fossilis; green

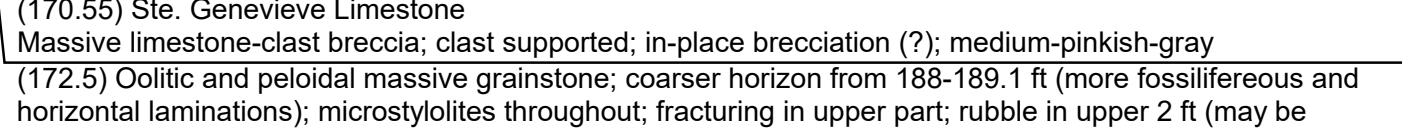

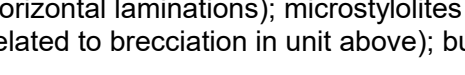




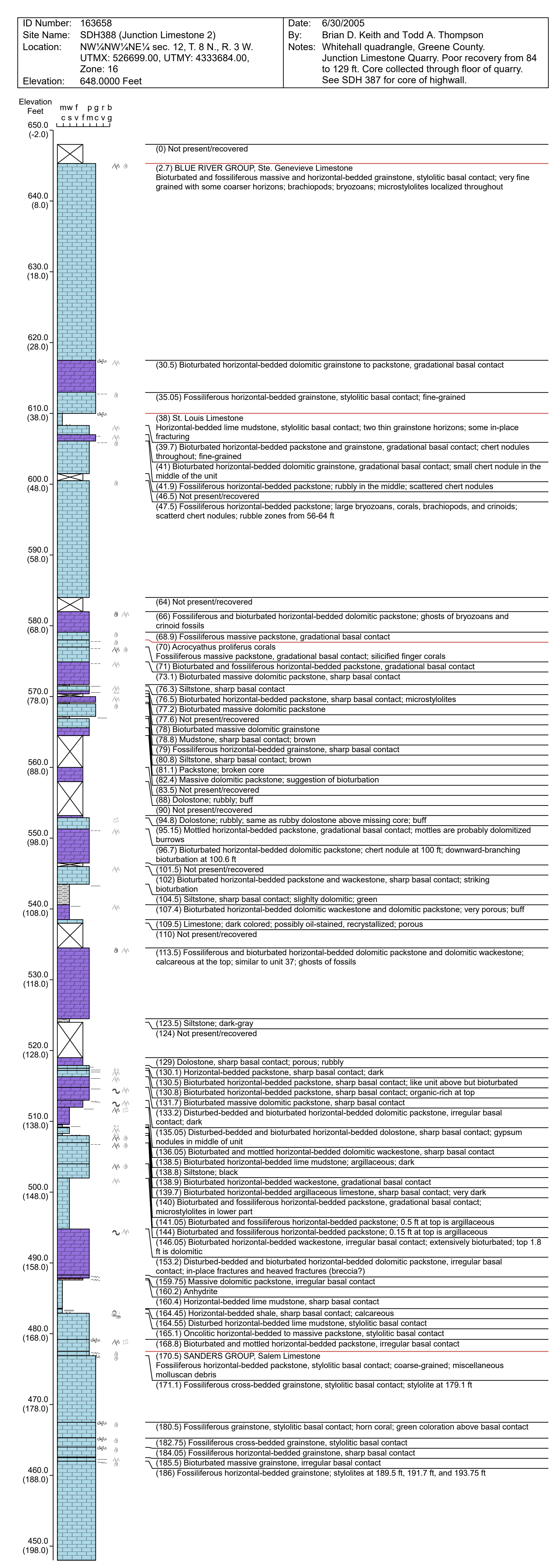




\begin{tabular}{|c|c|c|}
\hline \begin{tabular}{|l} 
IN N Number: \\
Site Name: \\
Location: \\
Elevation:
\end{tabular} & 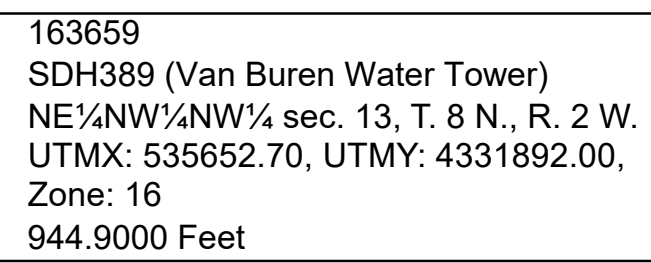 & $\begin{array}{l}\text { Date: } 711322005 \\
\text { By: } \text { Todd A. Thompson and Brian D. Keith } \\
\text { Notes: Bloomingon quadrangle, Monroe County }\end{array}$ \\
\hline
\end{tabular}

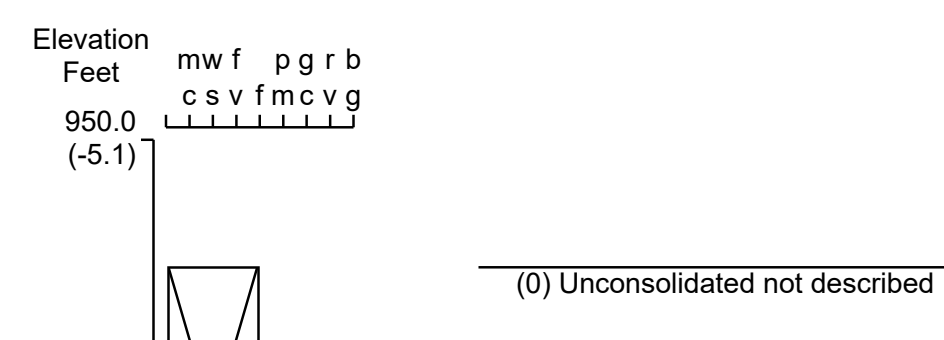

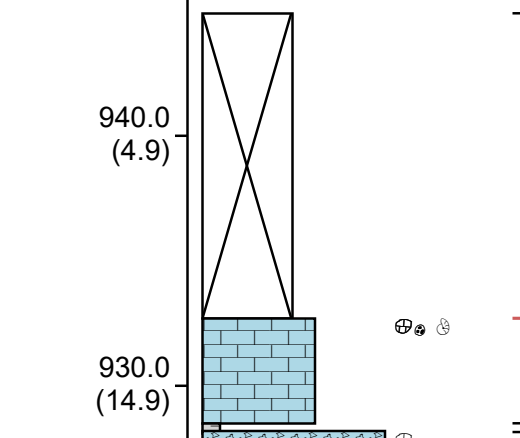
(12.2) BLUE RIVER GROUP, Paoli Limestone
Intraclastic and fossiliferous flaser-bedded packstone; coated grains, molluss shell debris; light-gray
\begin{tabular}{c}
920.0 \\
$(24.9)$ \\
\hline
\end{tabular}

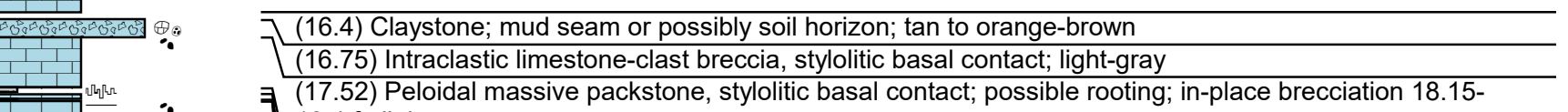

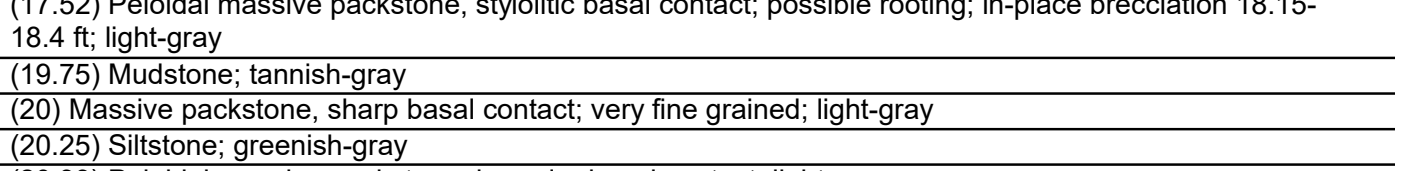

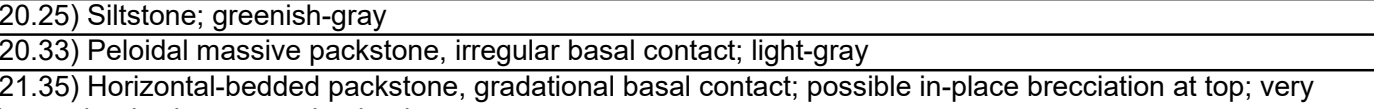

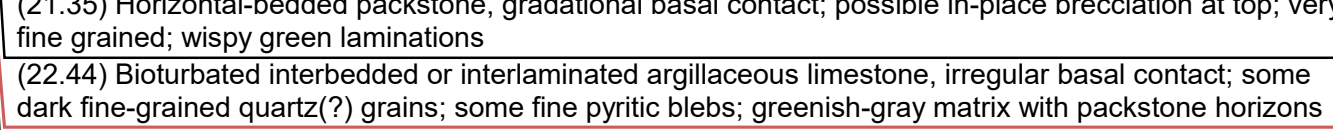

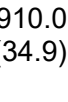

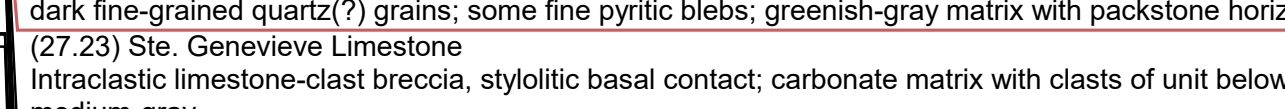

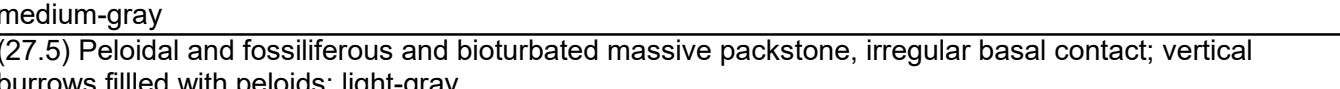
$(44.9)$

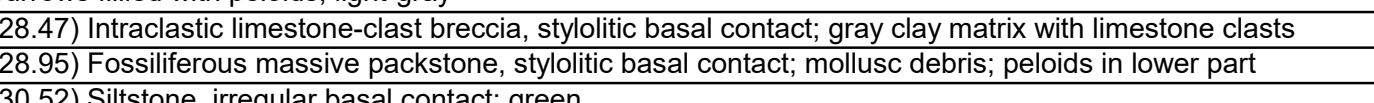
30.57) Fossiliferous massiviv packstone, sharp basal contact, green argillaceous wisps

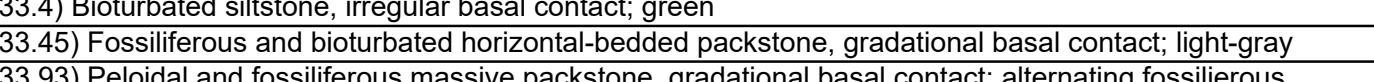

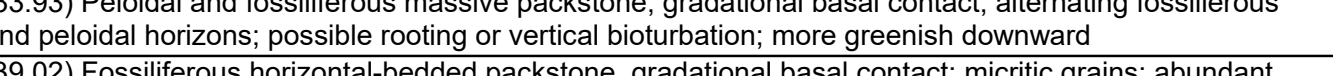
green argillaceous wisps
(39.6) Fossiliterous horizontal-bedded packstone, gradational basal contact; less argillaceous than

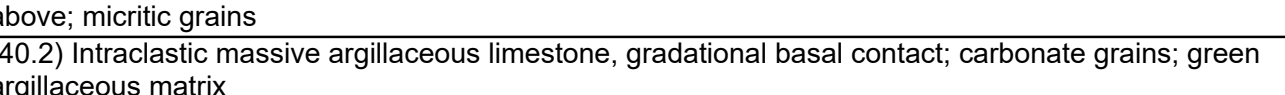 argillaceous matrix
(40.9) Horizontally-bedded packstone, irregular basal contact, micritized grains; argillaceous wisps; ilight-
greenish-gray
$(42.25)$-Fosiliferous massive argillaceous limestone, irregular basal contact; crinoids; greenish
$(42.6)$ Indian Creek Limestone Beds

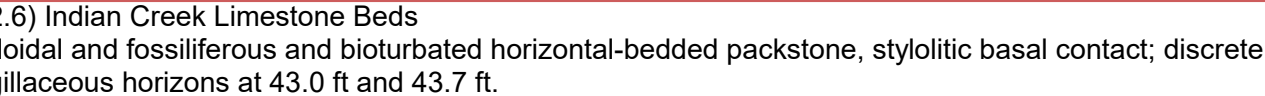

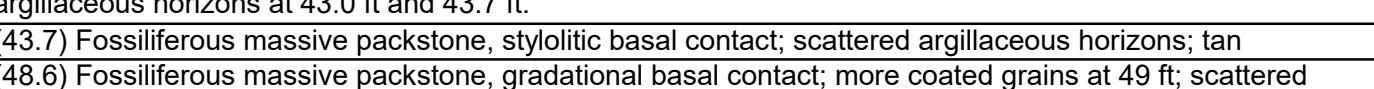

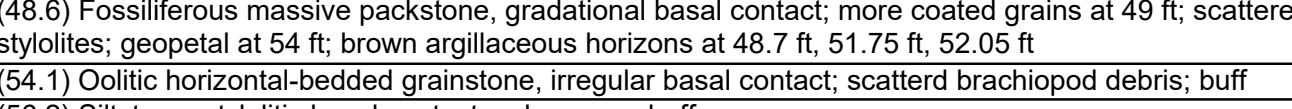

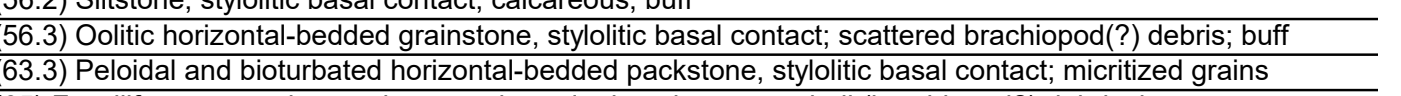 (65.8) Oolitic cross-bedded grainstone, sharp basal contact; quartz grains scattered throughout and
along lamina
$(77)$ Fossilifier rous massive grainstone, gradational basal contact; ; some horizontal bedding at the base;

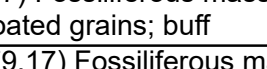 作

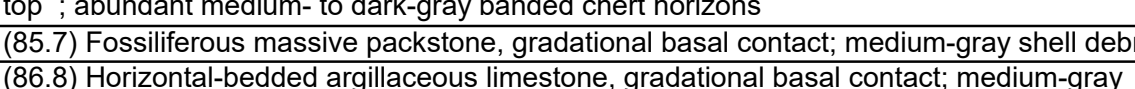 \begin{tabular}{l} 
(88.25) Massive packstone, gradational basal contact, microstylolites; medium-light-gray \\
\hline 89.95$)$ Bioturbated horizontal-bedded argillaceous limestone, gradational basal contact; medium-gray
\end{tabular}

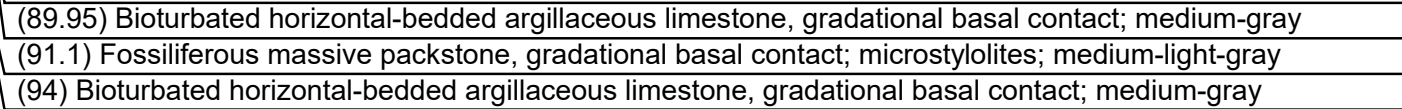

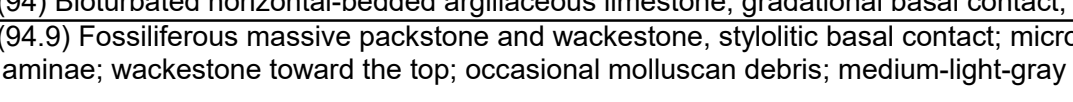

coarse skeletatal debris at at base to to horizontal finse-granined skeletelal deburis at the top top

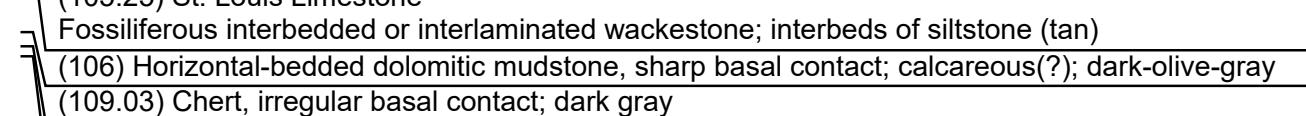

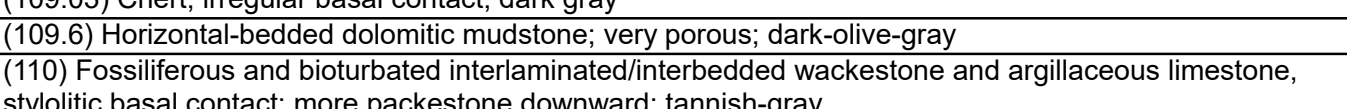

(118.9) Massive packstone, styllitic basal contact; chert nodule at 119.1 1t; medium-tannish-gray

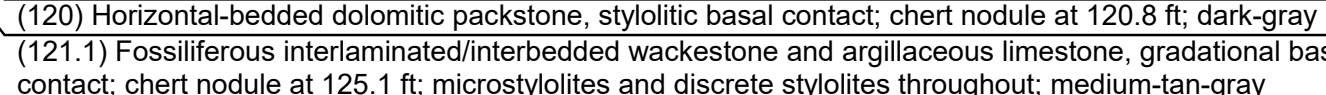

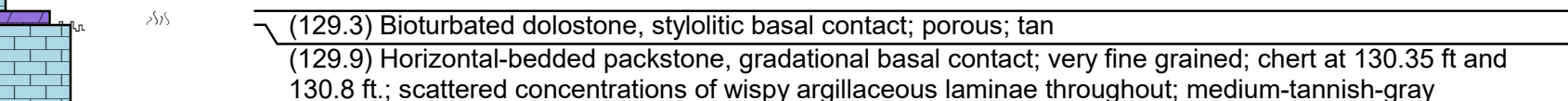

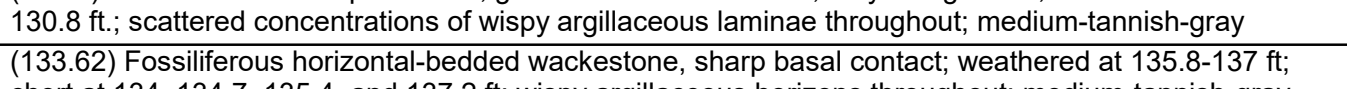

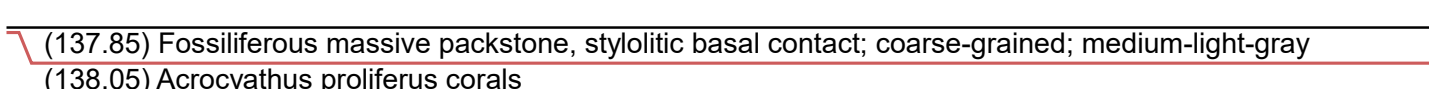
Fossiliferous massiviv packstone, irregular basal contact, very fine grained; chert at $138.5 \mathrm{ft}$; silicified
finger corals at $139.5 \mathrm{ft}$, medium-light-gray, weathered from $142-145.2 \mathrm{ft}$.

(146.15) Fossiliferous horizontal-bedded argillaceous limestone, gradational basal contact, grainier at (146.6-146.8 8 ittit scattered greee wisps; medium-dark-gray
medium-gray 


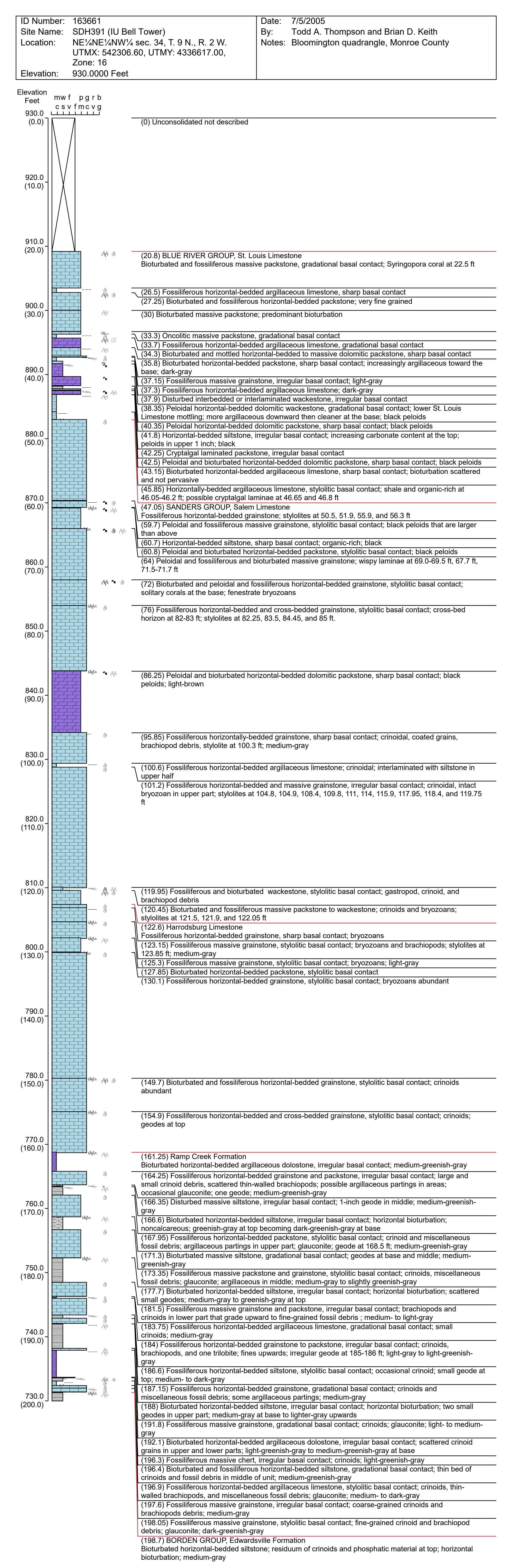




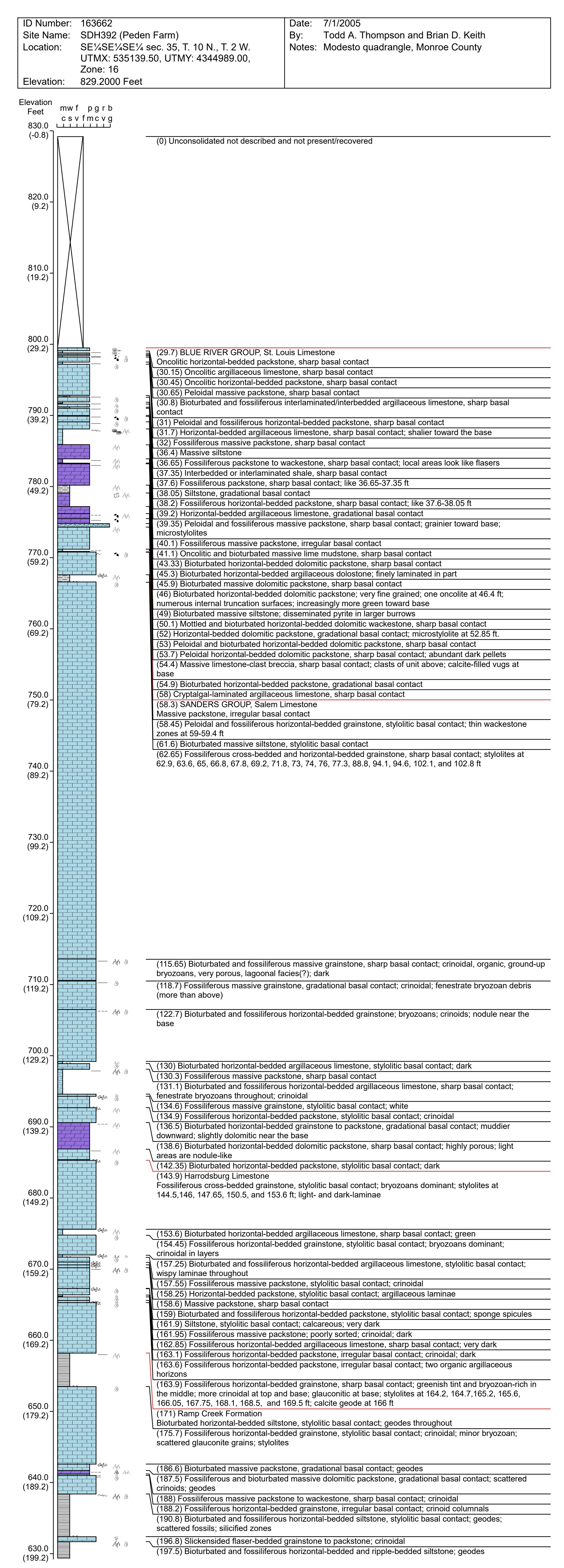




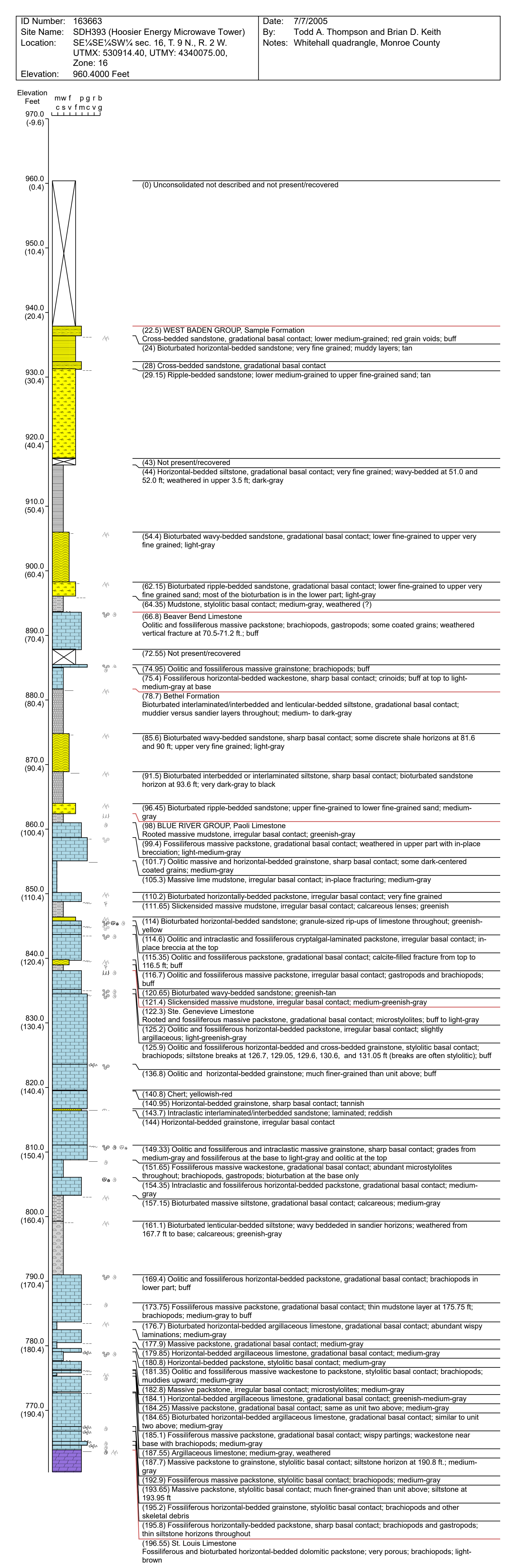




\begin{tabular}{|c|c|c|}
\hline $\begin{array}{l}\begin{array}{l}\text { ID Number: } \\
\text { Site enam: } \\
\text { Location: }\end{array} \\
\end{array}$ & 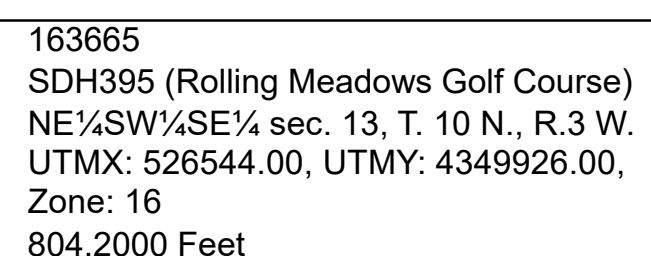 & $\begin{array}{l}\text { Date: } 6 / 28212005 \\
\text { By: } \quad \text { Brian } \mathrm{D} \text { Keith and Todd A. Thompson } \\
\text { Notes: Gosport quadrangle, Owen County }\end{array}$ \\
\hline
\end{tabular}

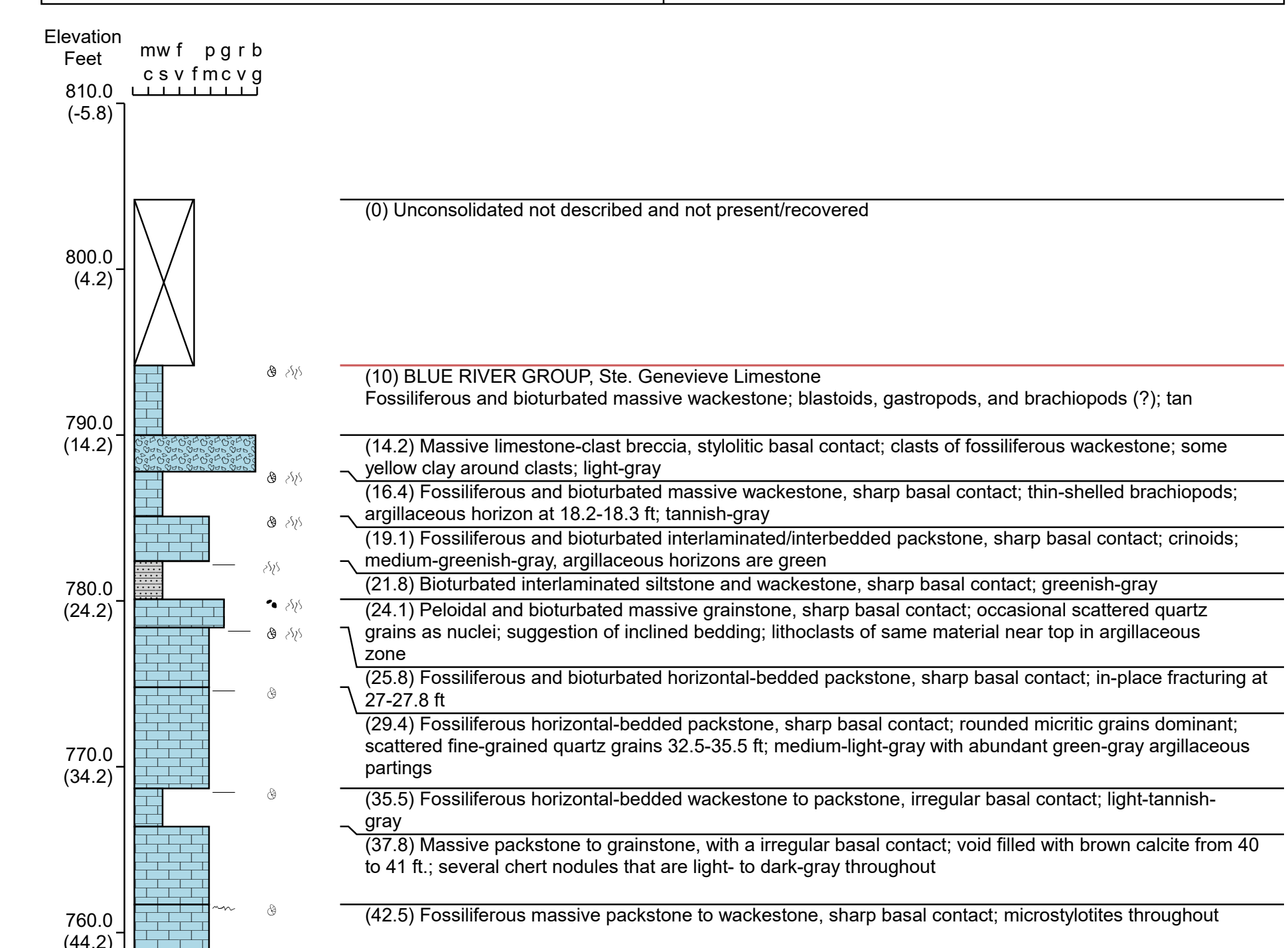

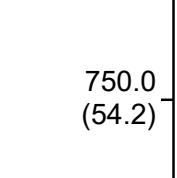

$\begin{array}{r}740.0 \\ (64.2) \\ \hline\end{array}$

wis o (62) Bioturbated and fossiliferous horizontal-bedded dolomitic packstone, with a sharp basal contact:

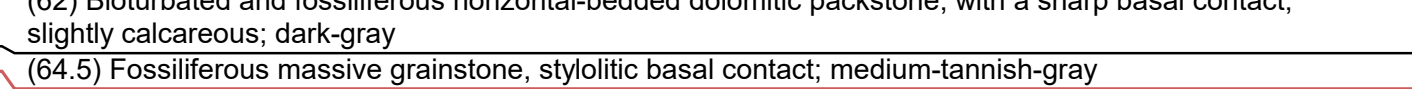
$\frac{(64.5) \text { Fossiliferous massive grainstone, styolitic basal contact; medium-tannish-gray }}{65.5) \text { St. Louis Limestone }}$ Massive lime mudstone, gradational basal contact; chert nodules from 67.8-68.3 ft; grainstone from 67.567.6 ti somer Bioturbated and fossiliferous horizontal-bedded dolomitic packstone; nodules have white chalky rind (devitrified chert); chert nodules trom 68.3-69 n throughout
(71.2) Fossiliferous interbeddded packstone and argillaceous limestone, irregular basal contact; ; rinoids
and brachiopods; green tint to fine-grained horizons (798) Fossilferous

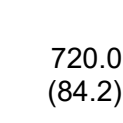

(87.3) Horizontal-bedded dolomitic mudstone, sharp basal contact; some green mud/glauconite/chlorite
blebs; ;reenish tint blebs; greenish titht

(90.7) Acrocyathus proliferus corals
Fossiliferous massive wackestone to packstone, sharp basal contact; microstyolitits; finger corals form

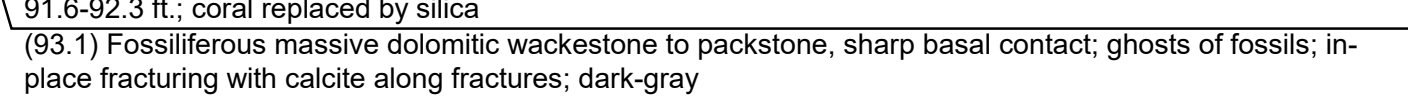

(98.8) Bioturbated massive dolomitic mudstone, sharp basal contact; some in-llace fracturing in the lower part
(101.9) Massive dolostone-clast breccia, sharp basal contact; clasts of dolomitic packstone to packstone; to green to maroon

(107.2) Horizontal-bedded dolomitic mudstone, sharp basal contact

(109.8) Bioturbated massive dolomitic packstone, sharp basal contact; scattered green blebs

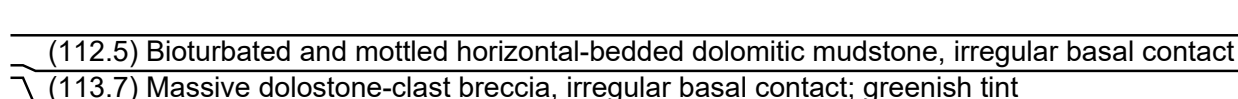

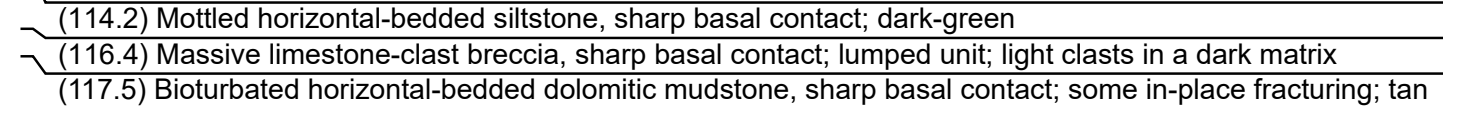

(123.2) Bioturbated horizontal-bedded lime mudstone, sharp basal contact; some in-place fracturing; dark (125.).3 Bitoturbated horizontal-bedded lime mudstone, sharp basal contact; darker and more argillaceous
near the base; chert nodules at the hase: (128.7) Bioturbated horizontal-bedded dolomitic mudstone, irregular basal contact (131.7) Massivive to horizontal-bedded dolostone-clast breccia, irregular basal contactiv vugs with brown -

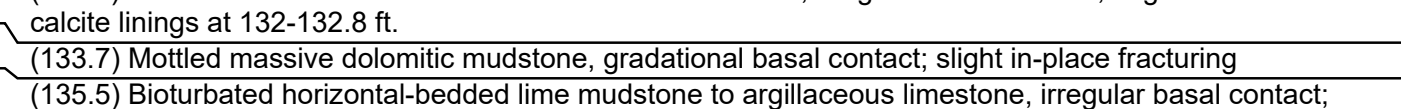
more ergililacoous toward the base
(137.2. Mottled horizontal-bedded lime mudstone, sharp basal contact

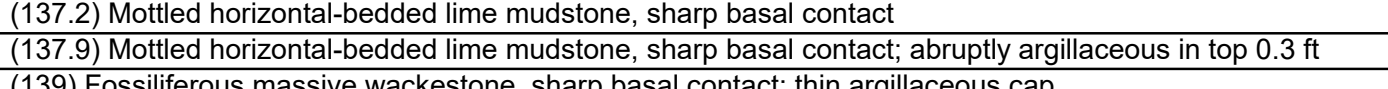
(139) Fossiliferous massive wackestone, sharp basal contact, thin argillaceous cap (142.7) Bioturbated massive dolomitic mudstone, sharp basal contact; calcareous in lower 0.7 ft; thin 0.1-1ft(146.1) Bioturbated horizontal-bedded lime mudstone, gradational basal contact; thin 0.1-1-t-thick (147) Fossiliferous and bioturbated horizontal-bedded dolomitic mudstone, gradational basal contact;

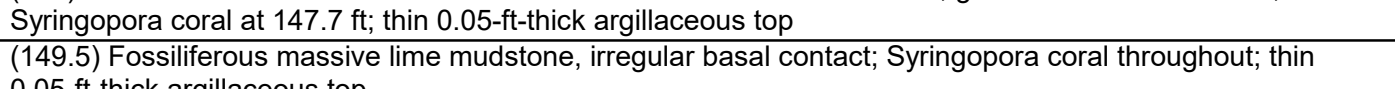
$.05-$-t-thick argillaceous top
194.9. Bioturbatated and mottled massive dolomitic mudstone, sharp basal contact, thin argillaceous (153.1) Bioturbated and mottled massive dolomitic mudstone, irregular basal contact; green

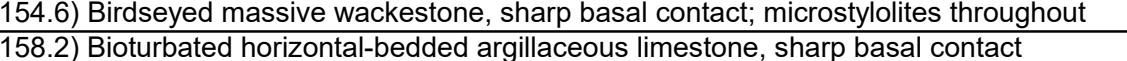

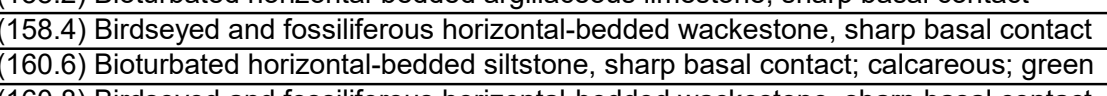
pward

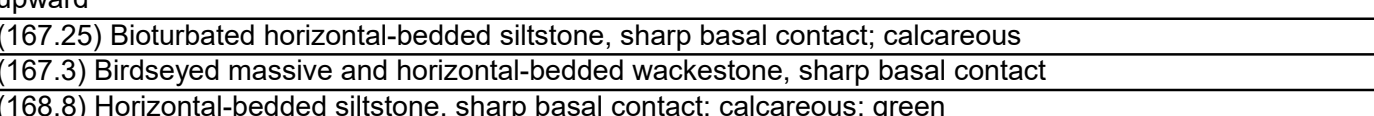

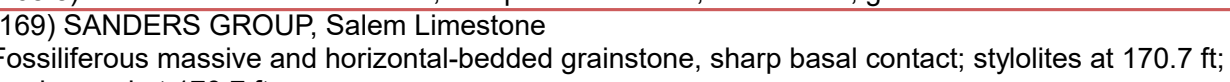

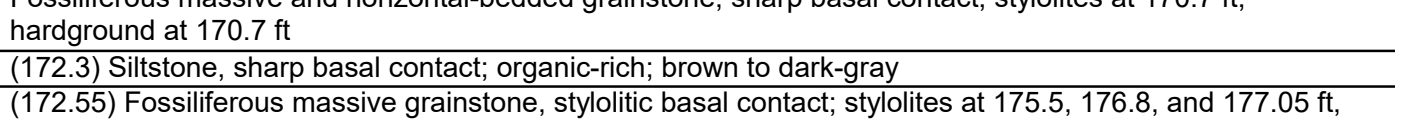
\begin{tabular}{l} 
hardground at $174.6 \mathrm{ft}$ \\
\hline 177.55$)$ Fossiliferous and bioturbated horizontal-bedded grainstone, sharp basal contact; stylolite at
\end{tabular}

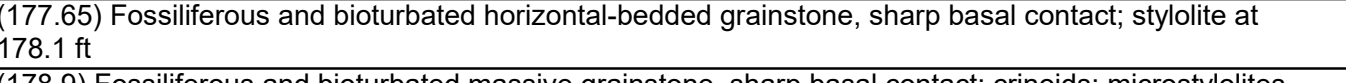

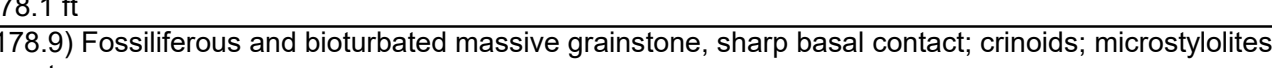

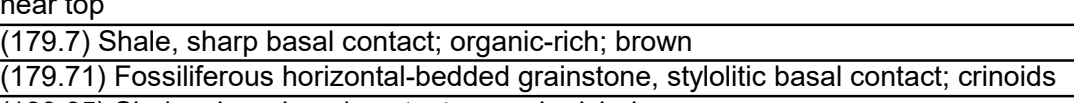

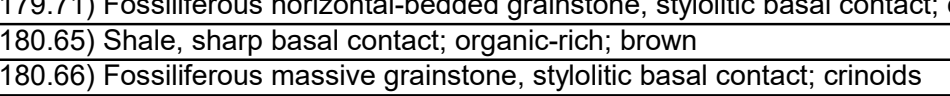
20.20.

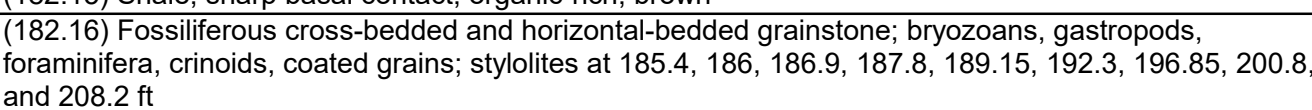




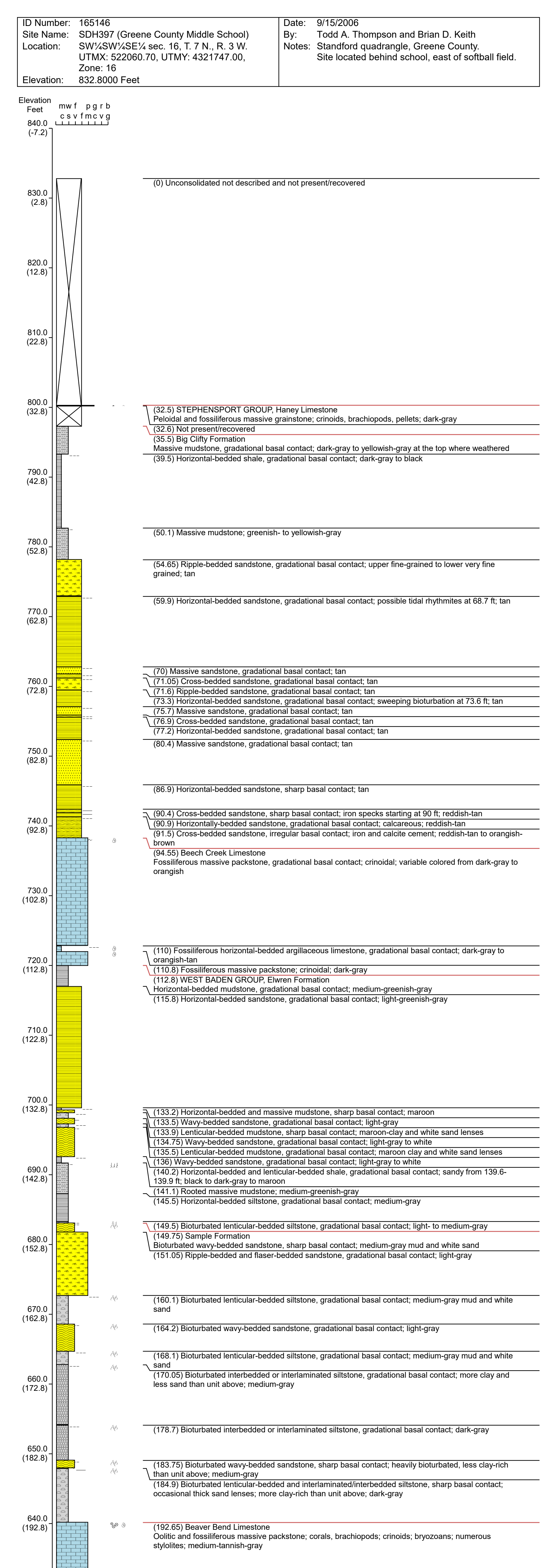


Date: 11/13/2006

SW'/ANE'/NNE'/4 sec. 33, T. 8 N., R. $3 \mathrm{~W}$

UTMX: 522343.10 UTMY: 4326969.00, Zone: 16
837.2000 Feet

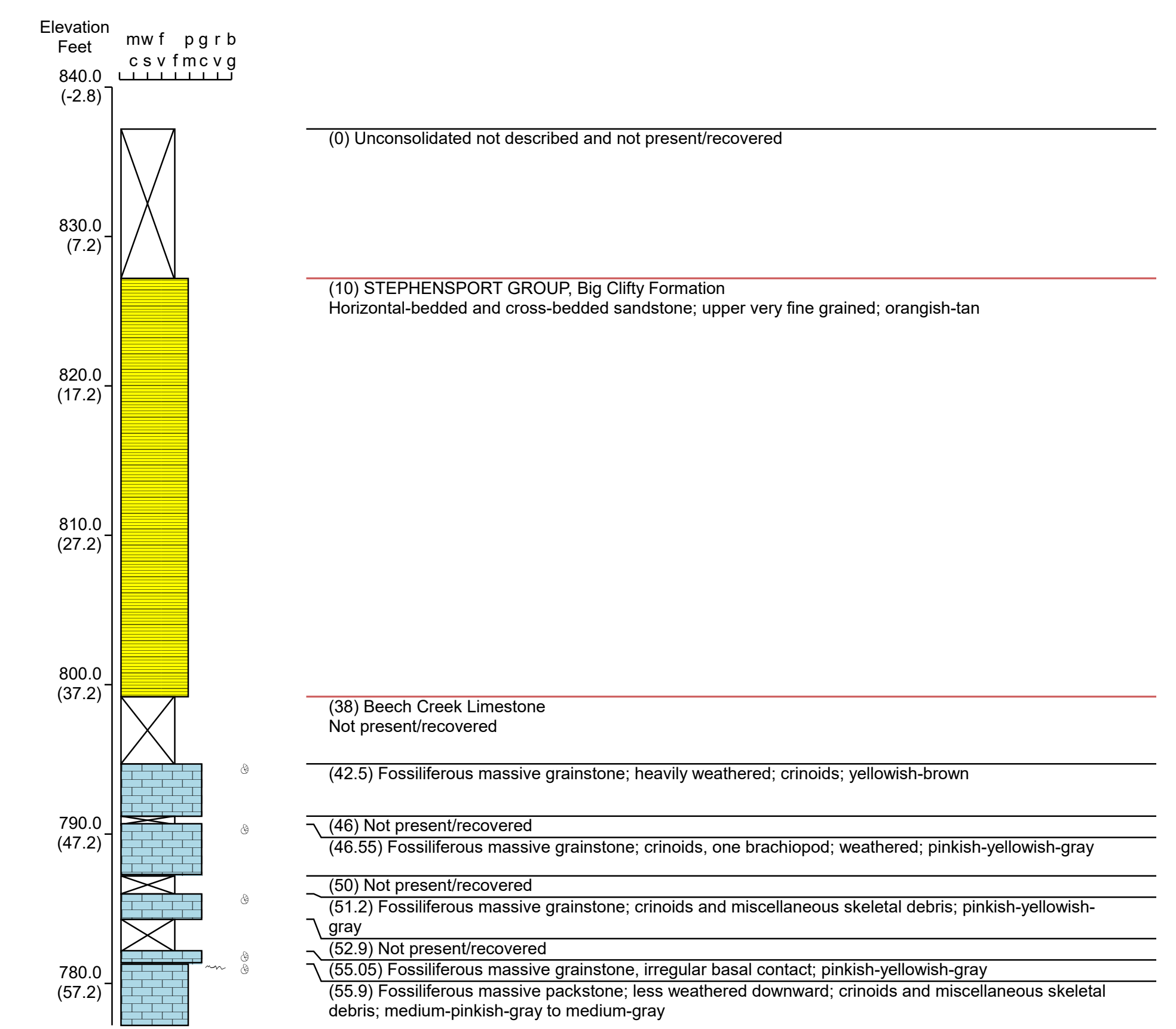

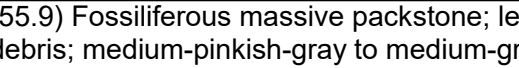




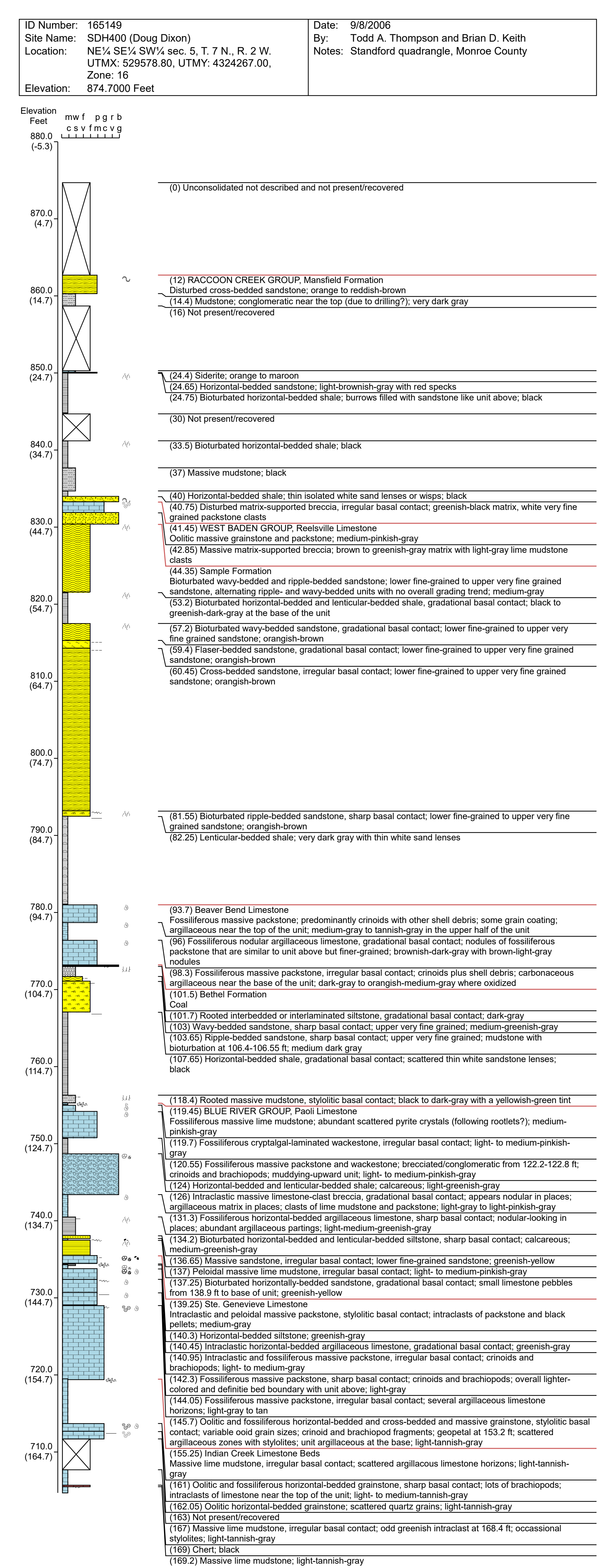




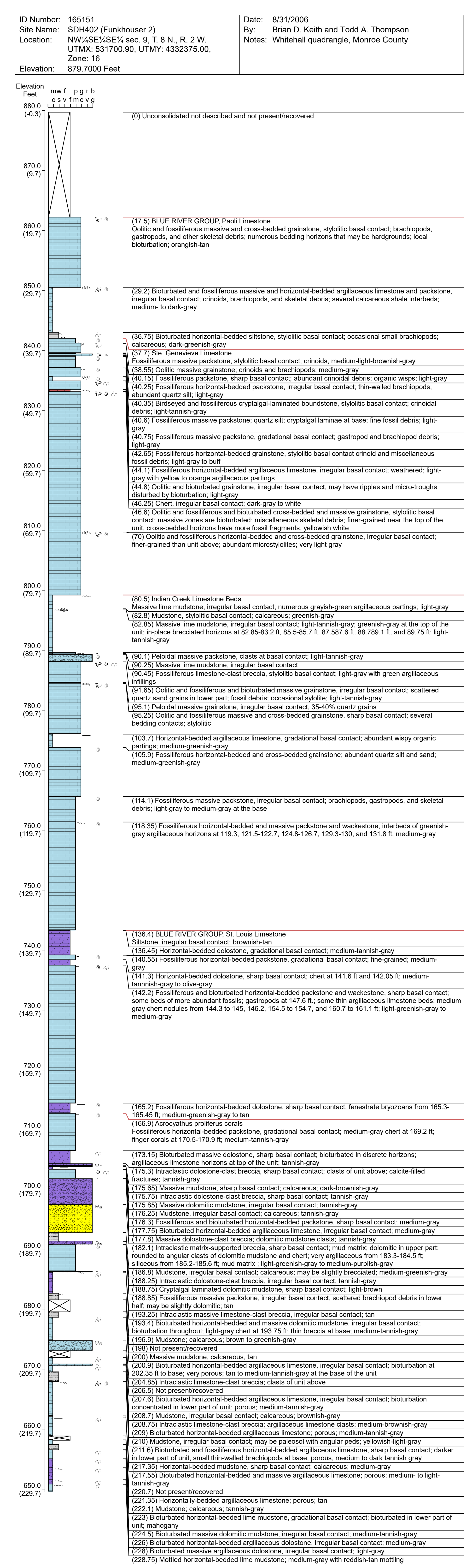




\begin{tabular}{|c|c|c|}
\hline \begin{tabular}{|l} 
ID Number: \\
Sitie Name: \\
Location:
\end{tabular} & 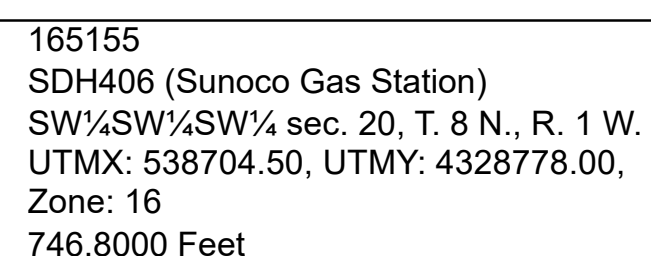 & $\begin{array}{l}\text { Date: } \\
\text { By: } 10 / 3012006 \\
\text { Brian D. Keith and Todd A. Thompson } \\
\text { Notes: Clear Creek quadrangle, Monroe County }\end{array}$ \\
\hline
\end{tabular}

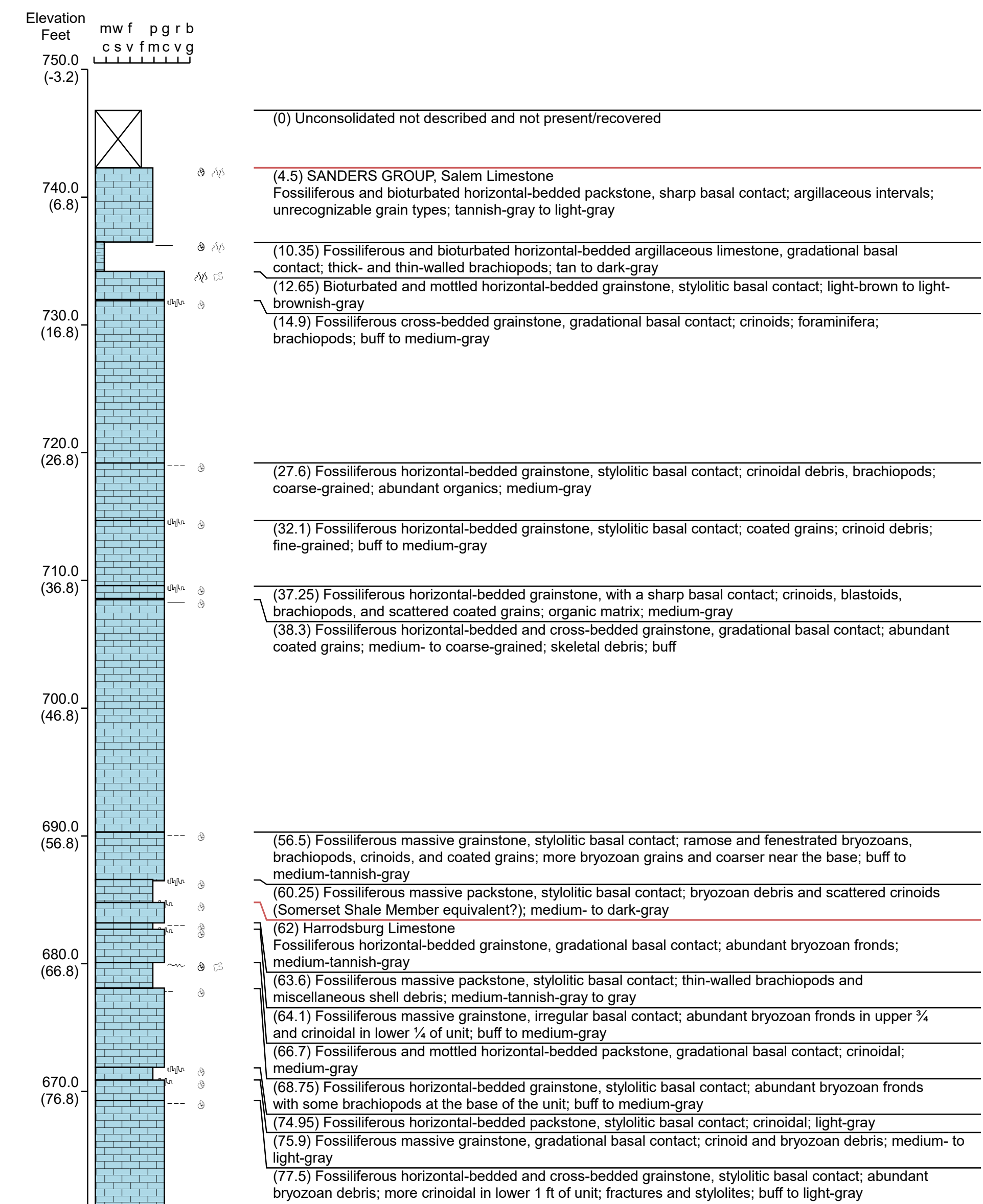

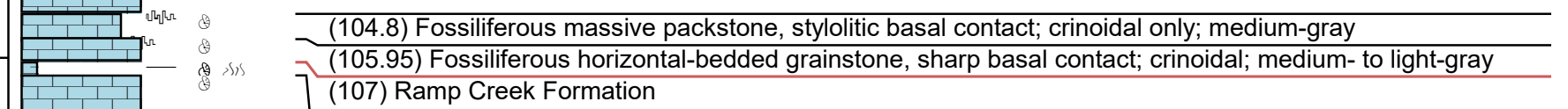

(98.75) Fossiliferous massive grainstone, stylolitic basal contact; crinoidal from top to $99.4 \mathrm{ft}$, then

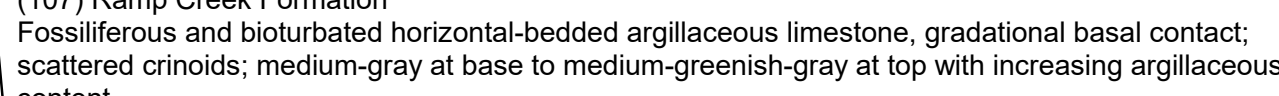

(107.6) Fossiliferous horizontal-bedded grainstone, irregular basal contact; abundant crinoids and
occasional bryozoans; increasing argillaceous partings toward base; medium-gray

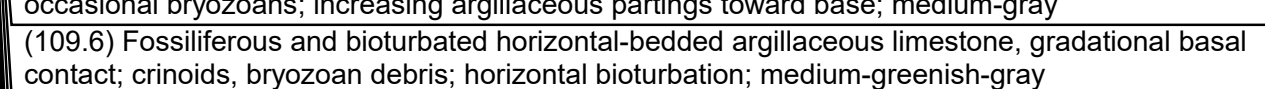

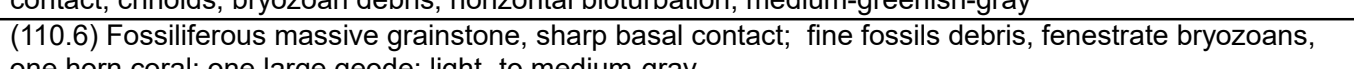

112.15) Fossilifererous horizontal-bedded argillaceous limestone, styolitic basal contact, scattered

crinoids; large geode at base; medium-gray
(114.5) Fossiliferous horizontal-bedded grainstone, sharp basal contact: fenestrate bryozoans, crinoids;

medium-gray
(114.9) Bioturbated and fossiliferous horizontal-bedded siltstone, irregular basal contact, fine fossil debris;

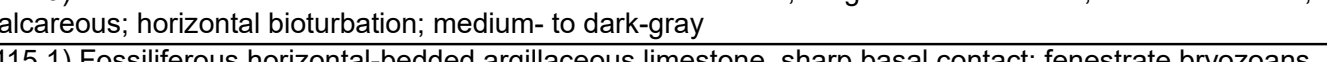

with argillaceous layers and occasional crinoids; one small geode; medium-gray
(117.2) Bioturbated and fossiliferous horizontal-beddded siltstone, gradational basal contact; slightly

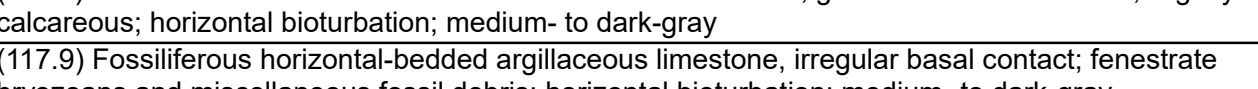

bryozoans and miscellaneous fossil debris, horizontal biturbation; medium-to dark-gray
$(118.8)$ Fossilifierous massive grainstone, irregular basal contact, fenestrate bryozzoans and

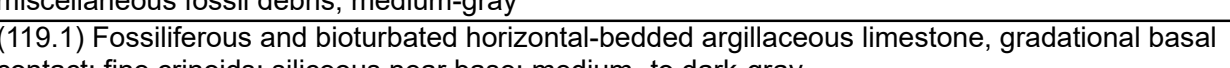

\begin{tabular}{l} 
contact, fine crinoids; siliceous near base; $m$ medium- to dark-gray \\
\hline 122.7$)$ Fossiliferous horizontal-bedded grainstone; very fine grained with argillaceous wisps; siliceous at
\end{tabular}

base; scatered manll logodes; medium-gray
122.2) Bioturbated horizontal-bedded argillaceous dolostone, irregular basal-contact; flattened horizontal

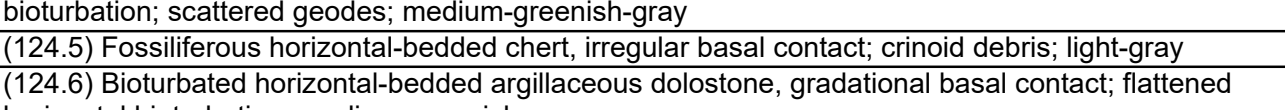

lorizontal bioturbation; medium-greenish-gray
(125.9) Fossiliferous horizontala-bedded argillaceous limestone, stylolitic basal contact, abundant crinoid

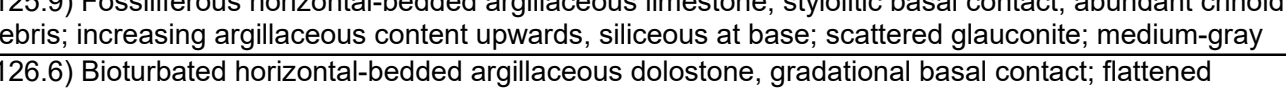

orizontal bioturbatition, occasional vertical burrow; scattered geodes; ighth to medium-greenish-gray

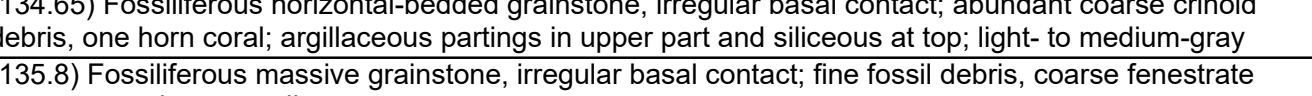

(1)yozoans at base; medium-gray
(137.05) Fossiliferous and bioturbated horizontal-bedded siltstone, styllititic basal contact; coarse fossill

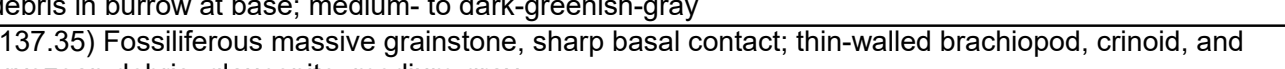

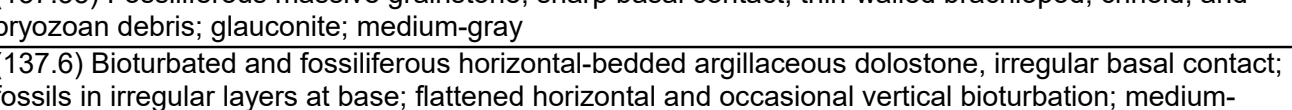

brownish-gray at base to greenish-gray at top
(139.3) Bioturbated and fossilfifrous horizontal-bedded argillaceous dolostone, irregular basal contact;

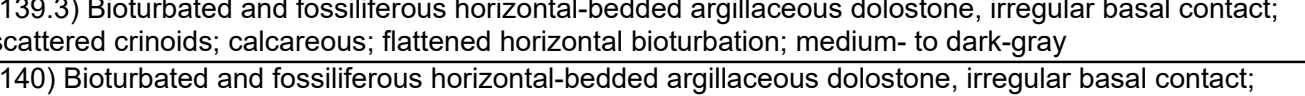

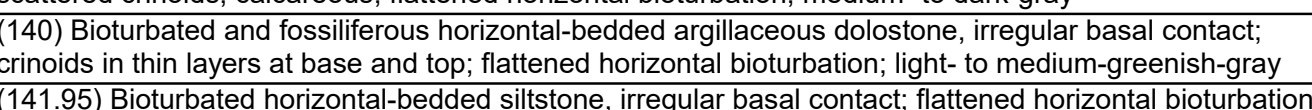

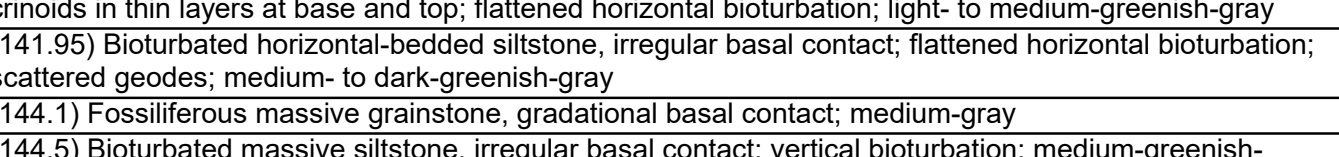

(144.1) Fossiliferous massive grainstone, gradational basal contact; medium-gray
(144.5)
grioturbated massive siltstone, irregular basal contact; vertical biturbation; medium-greeniish-

gray
(144.9) Fossiliferous massive grainstone, irregular basal contact, medium to fine crinoid and brachiopod

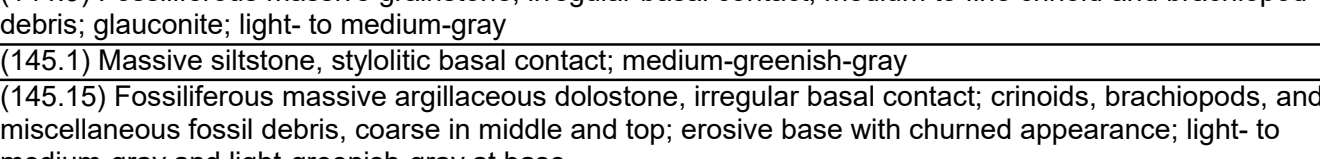

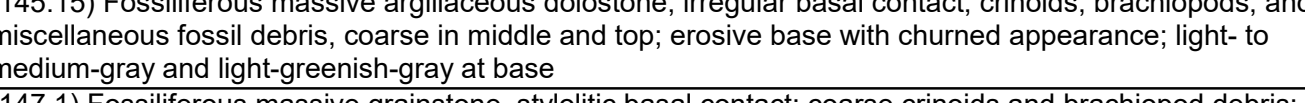

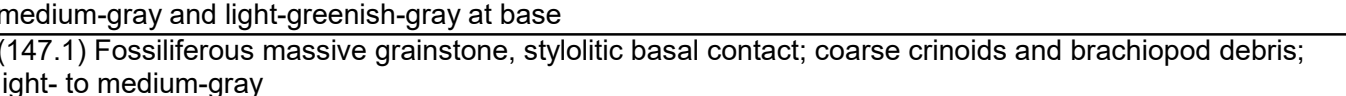
ght- to medium-gray
147 . 45) Fossififerous horizontal-bedded grainstone, styllititic basal contact; abundant coarse crinoids, $\frac{\text { brachiopod and bryozoan debris; siliceous at top; medium-gray }}{(148.55) \text { Bioturbated horizontal-bedded argillaceous dolos stone; flattened horizontal bioturbation; }}$ 


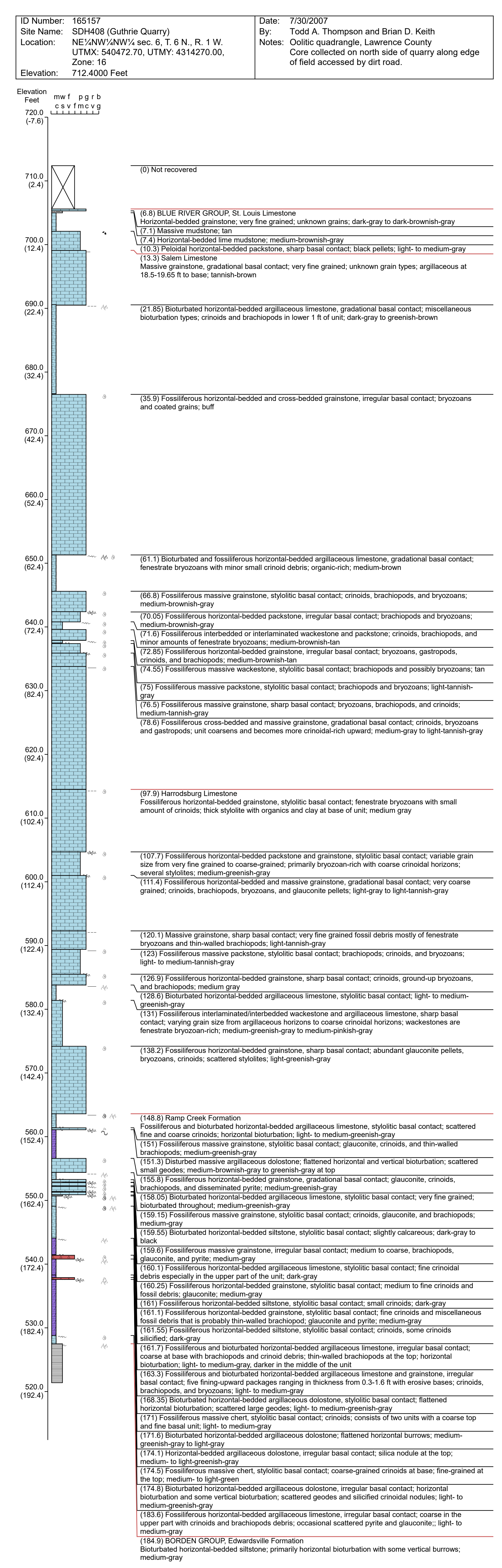




\begin{tabular}{|c|c|c|}
\hline \begin{tabular}{|l} 
IN N Number: \\
Site Name: \\
Location:
\end{tabular} & 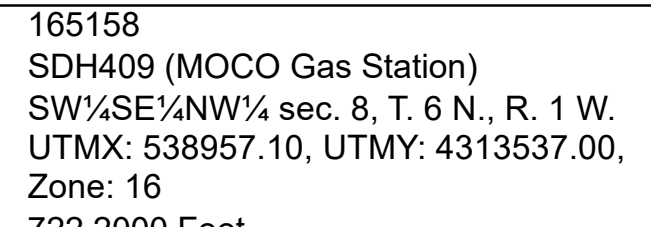 & $\begin{array}{l}\text { Date: } 81 / 122007 \\
\text { By: } \\
\text { Brian D. Keith and Todd A. Thompson } \\
\text { Notes: Oolitic quadrangle, Lawrence County }\end{array}$ \\
\hline
\end{tabular}
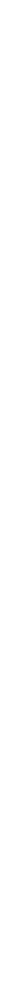

(24.2) BLUE RIVER GROUP, St. Louis Limestone
Bitoutubated horizontat-bedded packstone; very fine grained; medium-to dark-gray

$\frac{(24.85) \text { Mud; yellowish-ed }}{(26) \text { Bioturbated horizontal-bedded packstone; medium-gray }}$
(26.1) Not presentrotrecovorered

$\underset{\substack{6000 \\(322}}{322}$

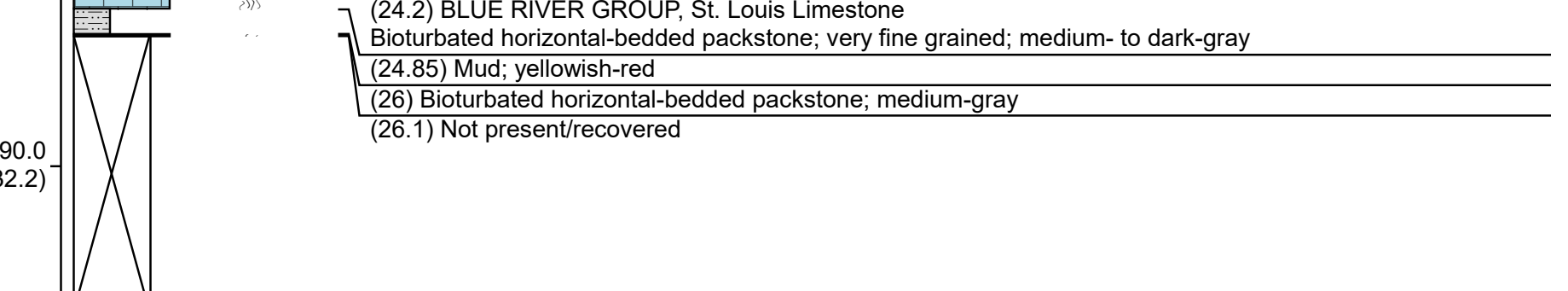

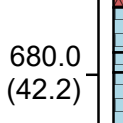

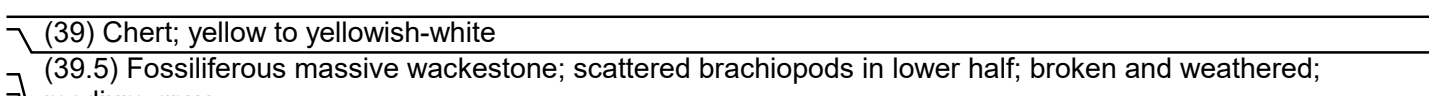

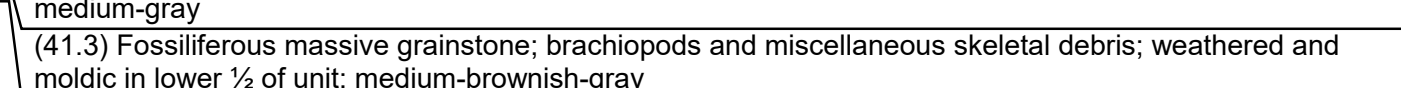

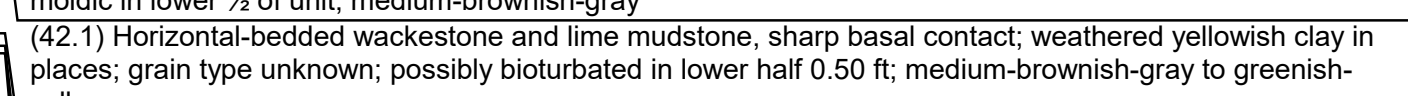

\begin{tabular}{l} 
yellow \\
\hline$(46.5)$ Bioturbated horizontal-bedded packstone; medium-grayish-brown \\
\hline 47 Mudi brown
\end{tabular}

\begin{tabular}{l}
$(47.3)$ Not presentrrecovered \\
\hline 51 ) Horizontal-bedded argillaceous dolostone, sharp basal contact; finely laminated; slightly calcareous;
\end{tabular}

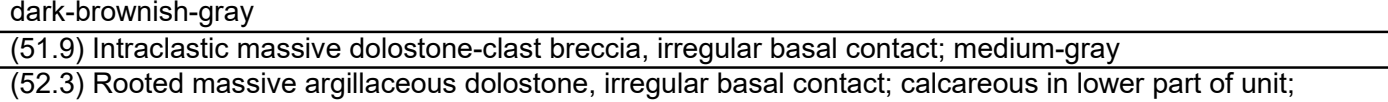

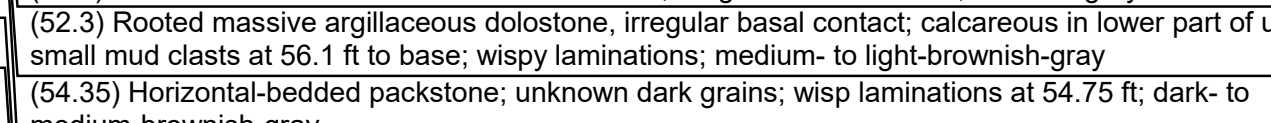

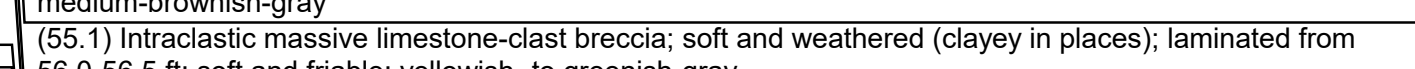

56.0-5.5.5tt: soff and friable; yellowish-to greenish-gray

(61) Bioturbated massive and horizontal-bedded dolomitic mudstone, gradational basal contact. fine-

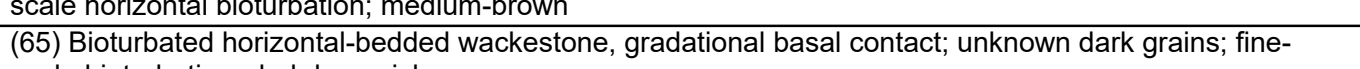

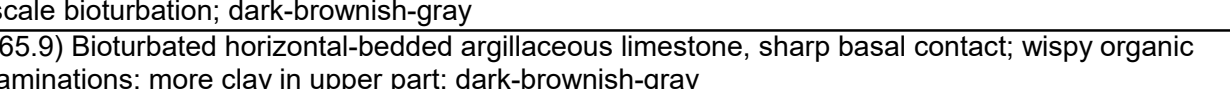

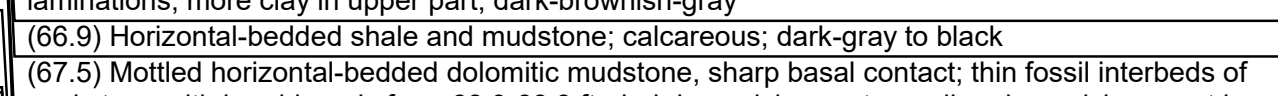

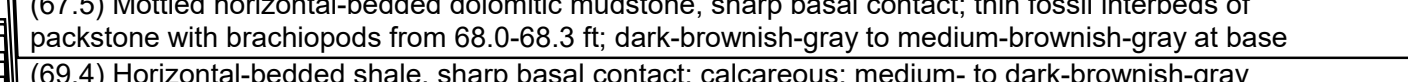

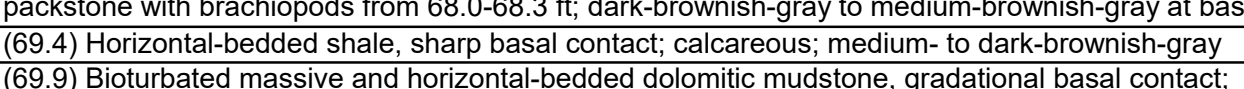

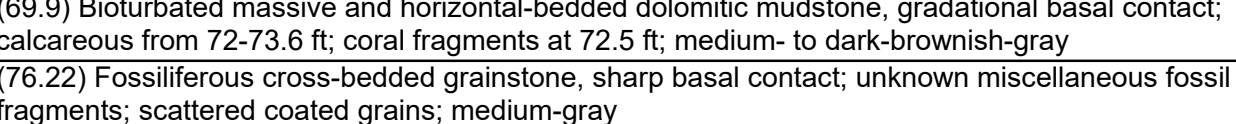

(79.3) Mudstone; dark-gray
$(79.5)$ Motled massive lime mudstone, irregular basal contact; bitutrbated; medium-brownish-gray

79.95) Rooted massive dolomitic mudstone, gradational basal contact, prominent rootlets; some clay
content, lilhht to medium gray

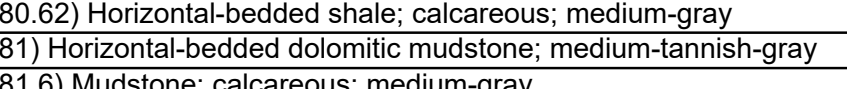

81.6) Mudstone; calcareous; medium-gray

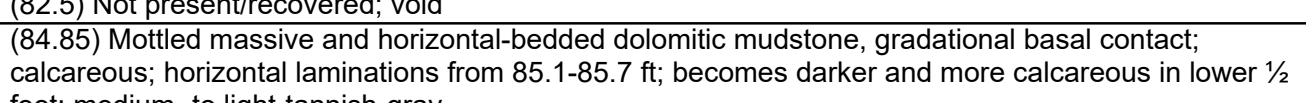

\begin{tabular}{l} 
(oot; medium- tolight-annish-gray \\
\hline 88 .22) Fossiliferous horizontal-bedded argillaceous limestone, gradational basal contact; skeletal debris;
\end{tabular}

\begin{tabular}{l} 
very fine grained; medium-to dark-brownish-gray \\
\hline 88.85 F Fossiliferous horizontal-bedded shale, gradational basal contact; calcareous; scattered fine
\end{tabular}

skeletal debris; dark-brownish-gray
(89.7) Motled massive and horizontal-bedded lime mudstone; broken in lower part; dark-brownish-gray

90.955 Mudstone; medium-gray
91) Bioturbated horizontala-bedded dolomitic mudstone, sharp basal contact, laminated with occasional

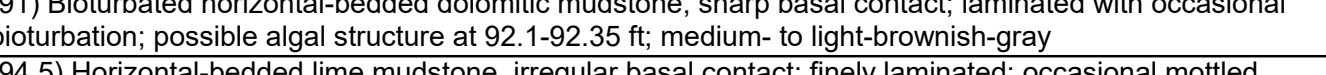

intervals, medium-tod dark-brownish-oray
(99.85) Motled and fossiliferous horzantal-bedded grainstone, styllitici basal contact, very fine grained;

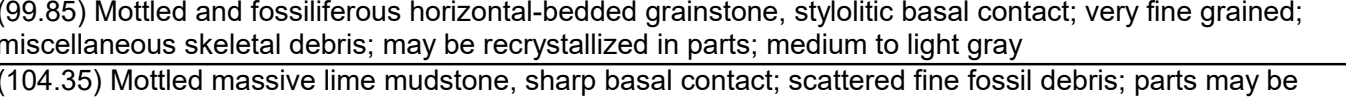

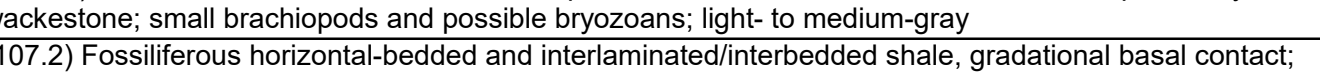

horeasing inclusions of packstone toward the base; black-to medium-gray
107.32) SANDERS GROUP Salem Limestone

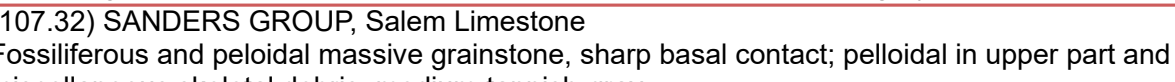

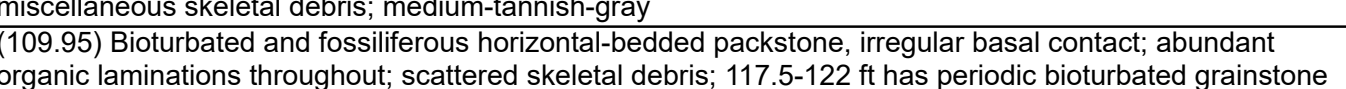

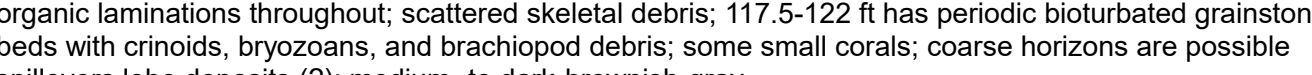

spillovers lobe deposits (?); medium-to dark-brownish-gray
(134.2) Fossilfifrous horizontala-bedded and cross-bedded grainstone, sharp basal contact; coated

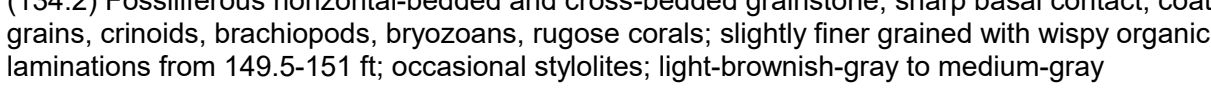

580.0
$(142.2)$

570.0
$(152.2)$

590.0
$(132.2)$
$(2)$

(155.7) Fossiliferous and bioturbated massive grainstone, sharp basal contact; bryozoan and crinoid . gradational basal contact; ghosts of scattered fossil debris; finely porouss; medium-brown
(1160.45) Bioturbated and fossiliferous massive packstone, sharp basal contact, brachiopods, bryozoans,

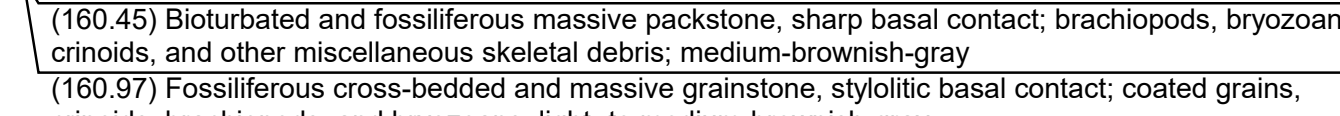
crinoids, brachiopods, and bryozoans; light-to medium-brownish-gray

(170.75) Fossiliferous massive and horizontal-bedded grainstone, gradational basal contact; coarsents
upward; brachiopods, bryozoans, corals, crinoids, gastropods; medium-brownish-gray to dark-gray 


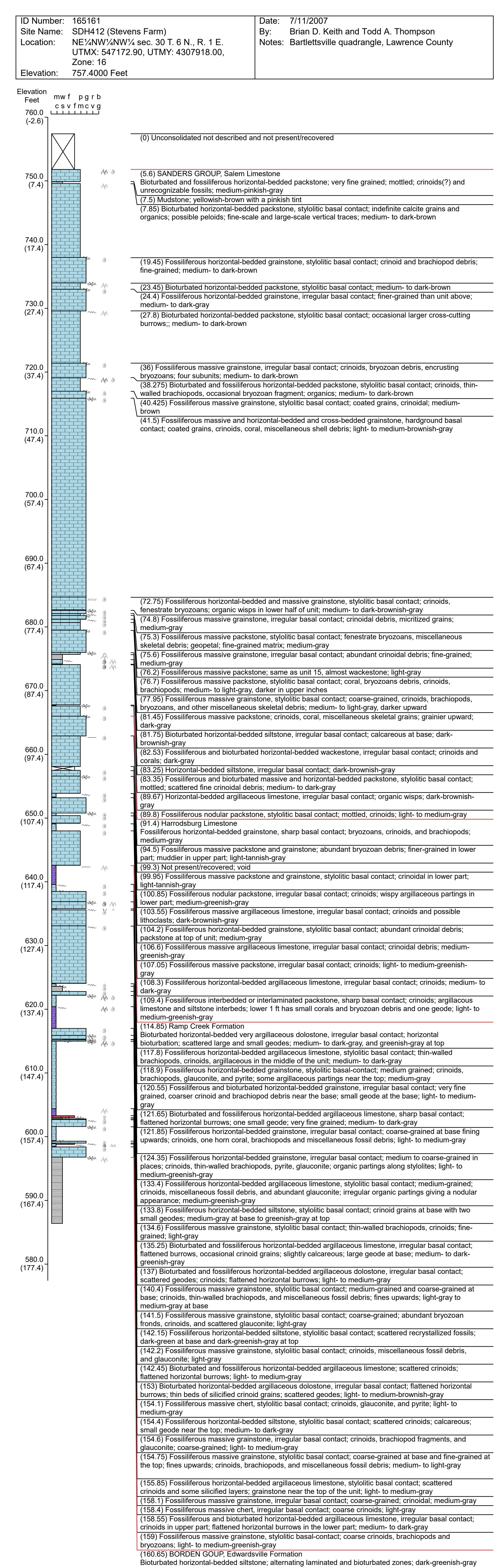




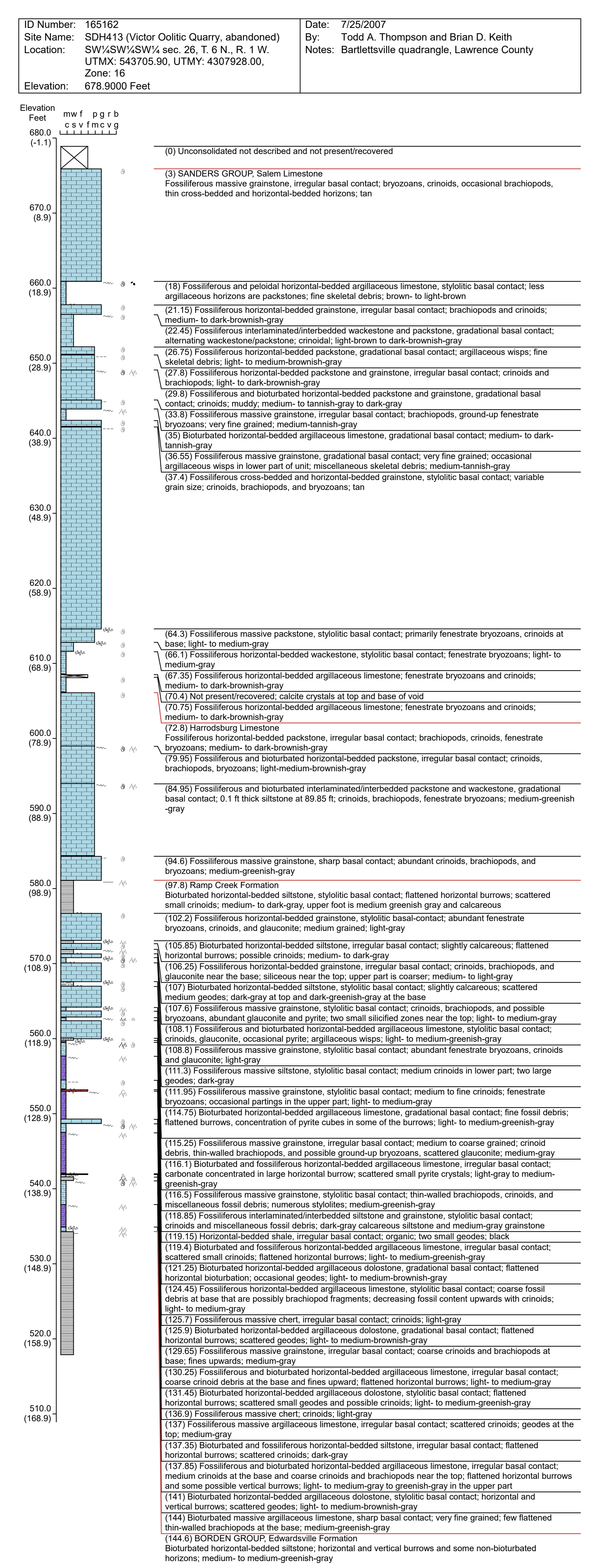




\begin{tabular}{|c|c|c|}
\hline \begin{tabular}{|l} 
IN N Number: \\
Site Name: \\
Location:
\end{tabular} & 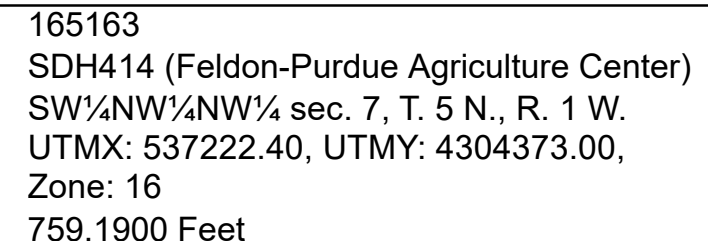 & 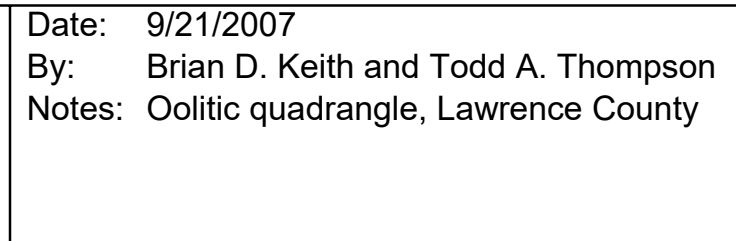 \\
\hline
\end{tabular}

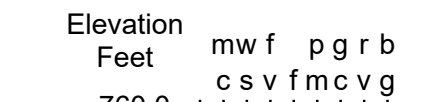

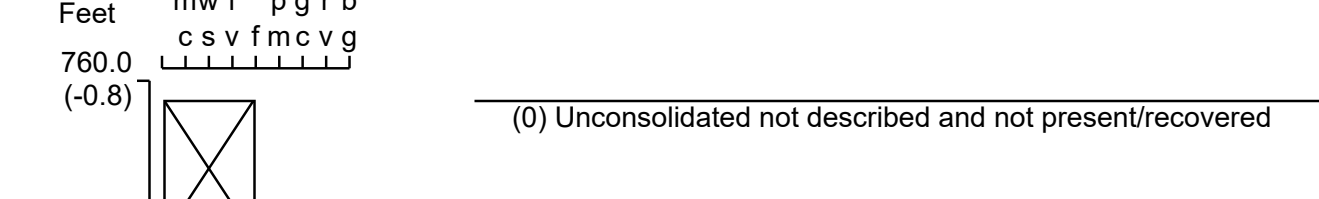
$\underset{750.0}{79.2)}$

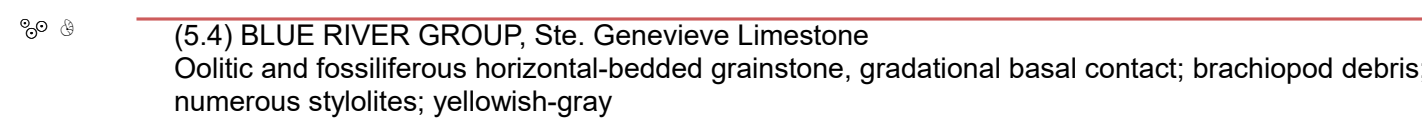

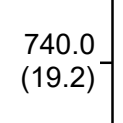
(19.7) Fossiliferous wackestone and lime mudstone, sharp basal contact; thin-walled brachiopods; light-
yellowish-gray
$\begin{array}{r}730.0 \\ (29.2) \\ \hline\end{array}$
720.0
$(39.2)$

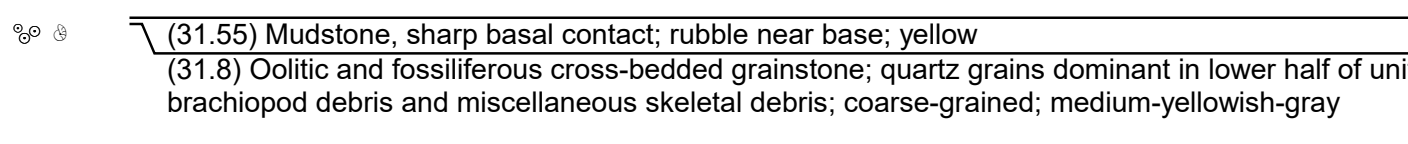
(39.9) Horizontal-bedded and massive lime mudstone, irregular basal contact; ligh-yellowish-gray
(43.1) Horizontal-bedded argillaceous limestone, sharp basal contact, wispy clay lamination in upper part of

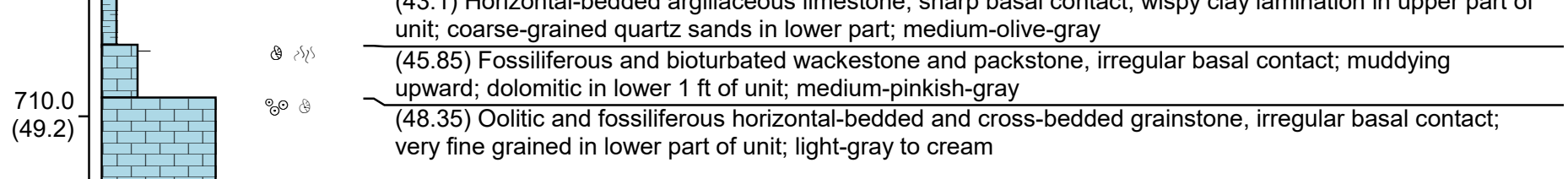
$\begin{array}{r}700.0 \\ (59.2) \\ \hline\end{array}$
$\underset{690.2)}{690}$
$\begin{array}{r}680.0 \\ (79.2) \\ \hline\end{array}$
670.0
660.0
$(99.2)$
$\begin{array}{r}650.0 \\ (109.2) \\ \hline\end{array}$
640.0
$(119.2)$
$\underset{(129.2)}{630.0}$
620.0
$(139.2)$
(59.8) St. Louis Limestone

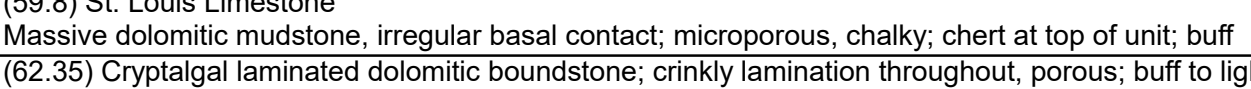

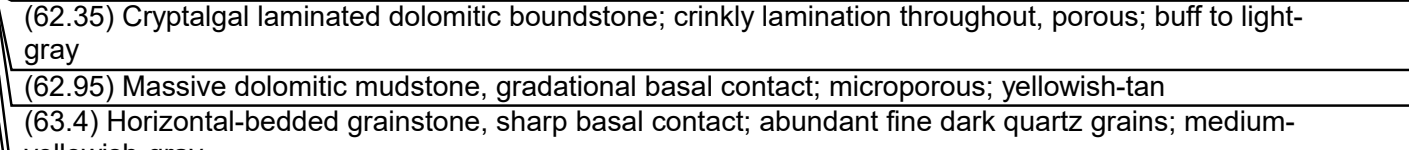 yellowish-gray
(65.6) Massive dolomitit mudstone, sharp basal contact; yellowish-tan
(67.5 Oolitic and fosilififrous massive and cross-bedded grainstone, sharp basal contact: unknown fort por units above and below; light to (696.9) Oolitic and fossiliferous massive and horizontal-bedded grainstone, irregular basal contact, finer-
grained in upper part with horizontal bedding; massive and coarser-grained in lower part very coarse

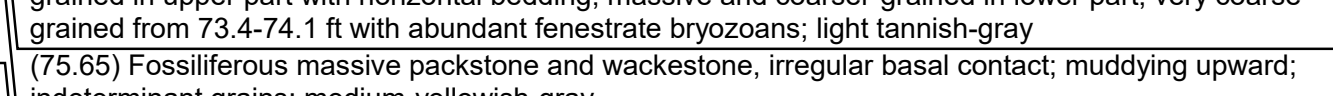 ind
indeterminant grains; medium-yellowish-gray

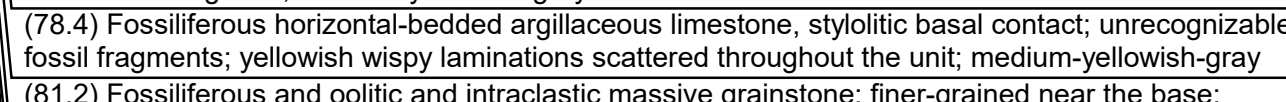 \begin{tabular}{l} 
brachiopod and miscellaneous fossil debris; medium-yellowish-gray \\
\hline 836 . Not presentrecovered
\end{tabular}
$\frac{1}{(85.7)}$ Fossilifierous and oolitic and intraclastic massive grainstone, styllitic basal contact; medium- (6).5) Massive lime mudstone; medium-brownish-gray
B7) Fossiliferous massive grainstone, stylolitic basal contact; brachiopods, gastropods, and fine skeletal

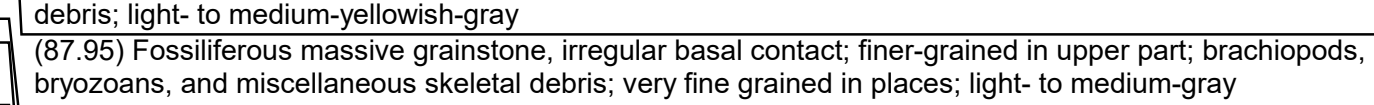 bryozoans, and miscellaneous skeletal debris; very fine grained in places; light- to medium-gray (96.75) Bioturbated and fossilifer rous massus (97.7) Massive dolomitic mudstone, irregular basal contact: ghosts of posible bioturbation: medium-

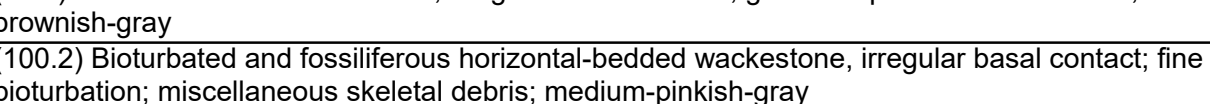
作

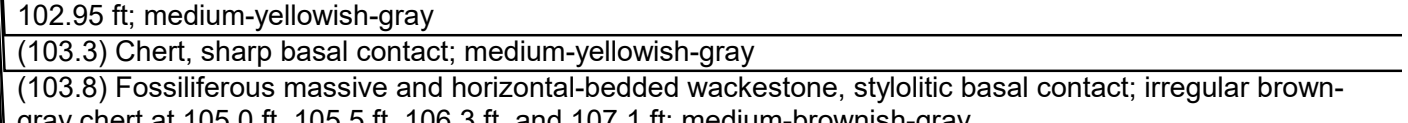 gray chert at $105.0 \mathrm{ft}, 105.5 \mathrm{ft}$, $106.3 \mathrm{ft}$, and $107.1 \mathrm{ft}$; medium-brownish-gray
(107.8) Fossilifferous massive lime mudstone and packstone, sharp basal contact; packstone at several

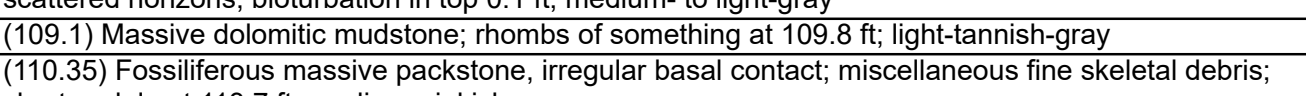

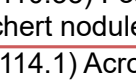

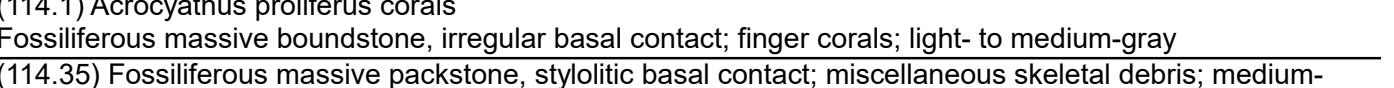

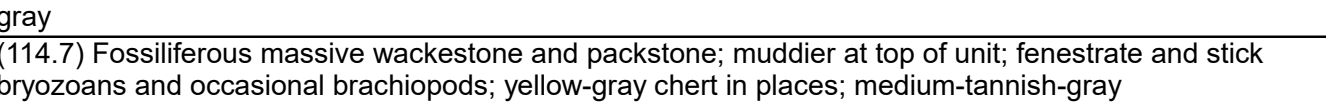
(21.7) Acrocoyathus proliferus corals
$122.6 \mathrm{ft}$; medium-tannish-gray
$(1237)$ Fosilfiferous
(123.7) Fossiliferous horizontalll-bedded argillaceous limestone, irregular basal contact; more
argillaceous near top miscellaneous skeletal d debris; medium-gray to vellowish-oray

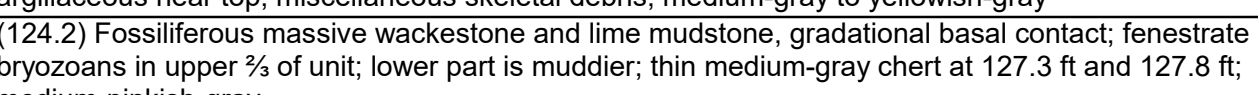

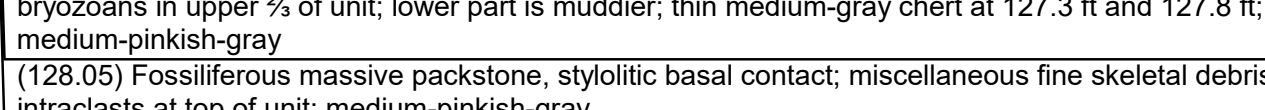

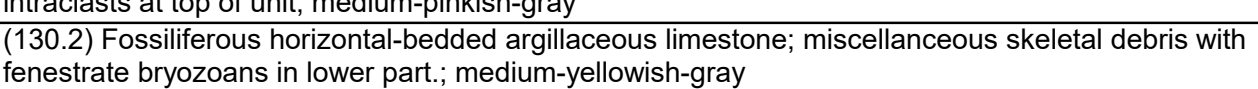




\begin{tabular}{|c|c|c|}
\hline $\begin{array}{l}\text { I N Number: } \\
\text { Site Name: } \\
\text { Location: }\end{array}$ & 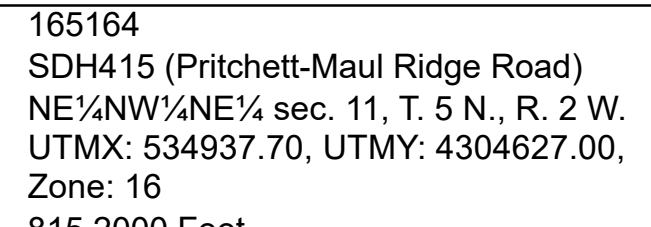 & 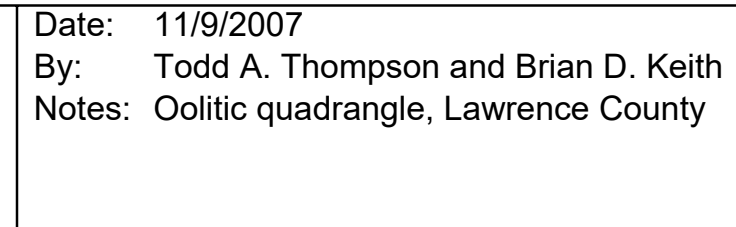 \\
\hline
\end{tabular}

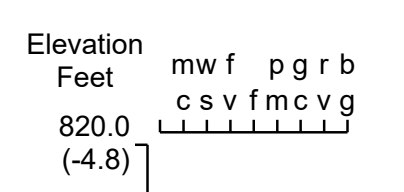
$\left.{ }^{(-4.8)}\right] \quad$ (0) Unconsolidated not described and not presentreceovered
${ }_{(5.2)}^{810.0}$
(1)
800.0
$(15.2)$
(25.2)
(35.2)
770.0
(29) WEST BADEN GROUP, Sample Formation
Horizontal-bedded shale; black
(31) Not presentrecevered
(38.5) Massive mudstone; black
$7(40.7)$ Sandstone; lower fine-grained to upper very fine-grained; dark-gray
(43) Beaver Bendd Limstonen
Gypsum, iregular far basal contact; fibrous crystalls; clear

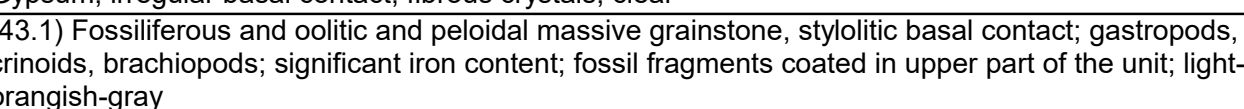

(53.4) Fossiliferous and oolitic and peloidal massive grainstone, irregular basal contact, argillaceous in

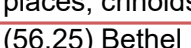

Horizontal-bedded shale, gradational basal contact; black

750.0
$(65.2)$
$(5)$

40.0
$(55.2)$
$(4)$

$\begin{array}{r}730.0 \\ (85.2) \\ \hline\end{array}$

\begin{tabular}{l}
720.0 \\
(95.2) \\
\hline
\end{tabular}

$\begin{array}{r}710.0 \\ (105.2) \\ \hline\end{array}$

700.0
$(115.2)$
-

690.0
1125.2
-10

680.0
$(135.2)$

670.0
(145.2)

650.0
$(165.2)$

(1) (66.9.9) Massive mudstone, gradational basal contact, dark-greenish-gray
$68.6)$ Massive and lenticular-bedded sandstone, irregular basal contact; very fine grained to upper very

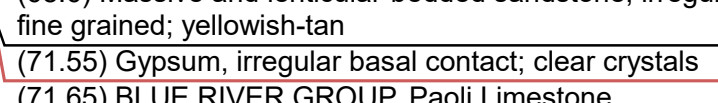

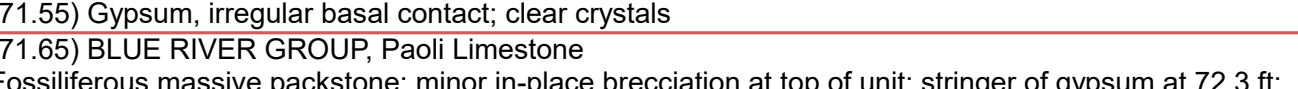

$\frac{\text { miscellaneous fossi debris; pinkish-gray }}{(75.9) \text { Oolitic and fossiliferous massive grainstone, irregular basal contact; crinoidal debris; medium-gray }}$

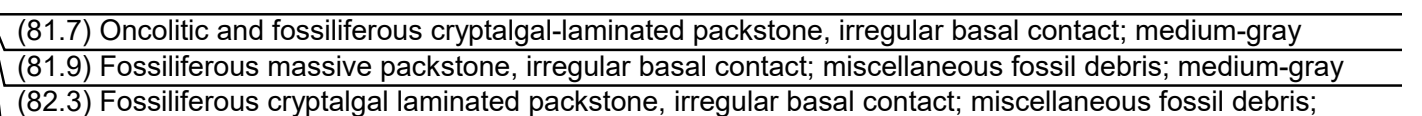

medium-gray
(82.9) Fossiliferous horizontal-bedded wackestone, gradational basal contact; miscellaneous fossil

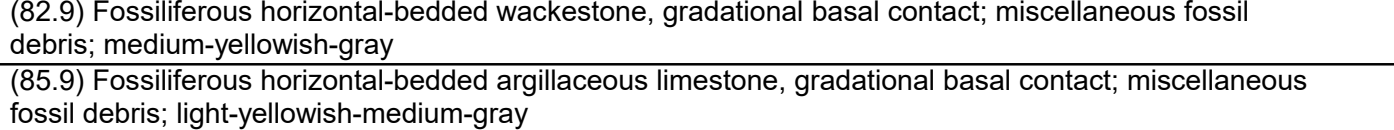

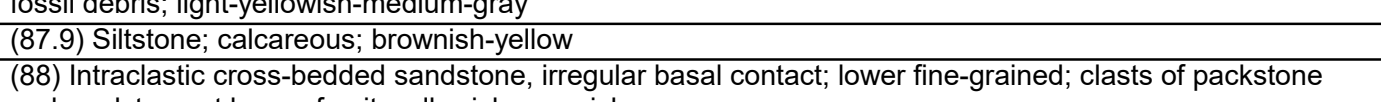

and mudstone at base of uniti yellowish-gre

\begin{tabular}{l} 
Fossiliferous cryptalagal-laminateded grainstone; crinoidal; medium-tannish-gray \\
\hline 90.3$)$ Fossiliferous massive packstone, gradadtional basal contact; in-place brecciation at top;
\end{tabular}

miscellaneous fossil debrisi, ight-yellowish-tan
(96.9) Fossilfifrous massive grainstone, styllitic basal contact; miscellaneous shell and crinoid debris;

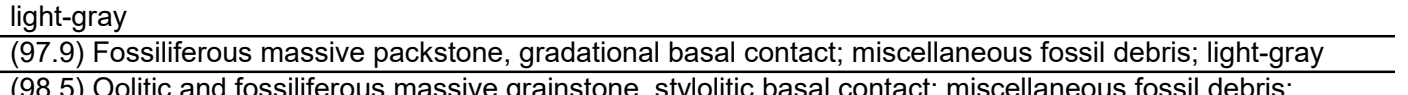

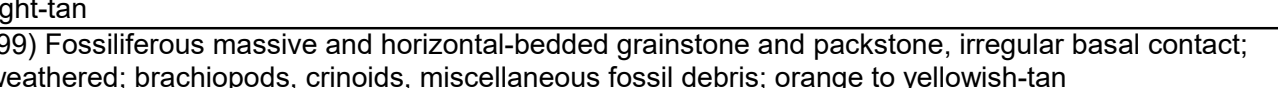

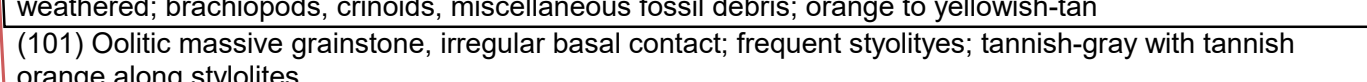

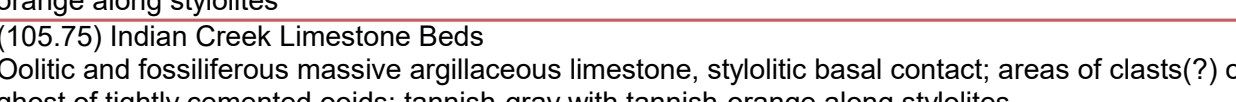

(109.8) Oolitic and fossiliferous massive grainstone, styllolitic basal contact, tightly cemented; tannish-

(11004) Fossiliferous massive and horizontal-hed

a
emented ooids concentrateded in layers: light- to medium-gray

124.2) Fossiliferous and oolitic massive grainstone, gradational basal contact; thin-walled brachiopods

and scattered zones of oolites; two thin lime mudstone layers; light-gray
$(129.65)$ Ooliticic and fossiliferous cross-beddded grainstone, sharp basal contact; coated miscellaneous

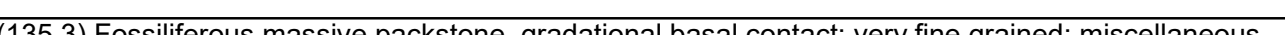

$\frac{1 \text { fossil debris, medium-gray }}{\text { (137.7) Fossilferious horizontal-bedded argillaceous limestone, gradational basal contact; crinoid debris; }}$

- medium-yellowish-gray $(140.5)$ (olitic massive grainstone; some scattered fossil debris; ight-gray

(144.3) Oolitic massive grainstone; scattered well-rounded upper to lower medium-grained quartz grains

(147.2) Intraclastic massive dolomitic grainstone, irregulary basal contact; scattered quartz grains; dark-

(147.95) Oolitic and fossiliferous massive grainstone colnd

olites near the

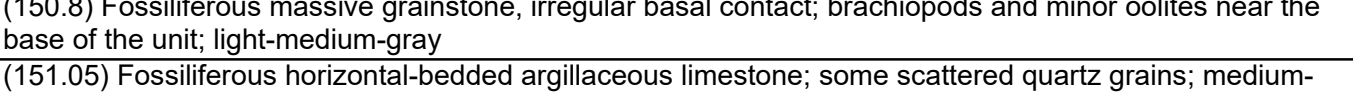

gray

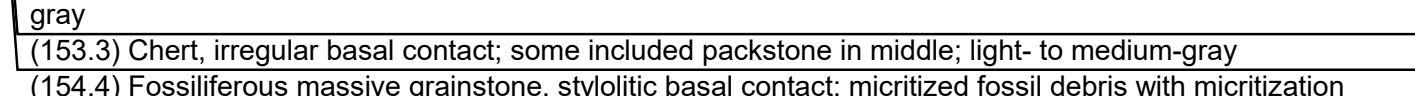

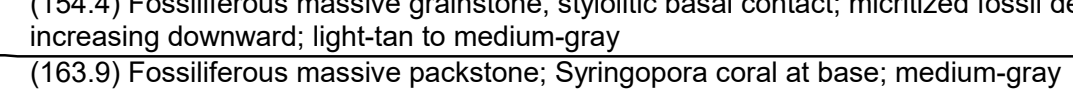




\begin{tabular}{|c|c|c|}
\hline 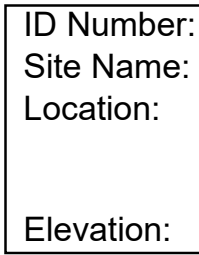 & 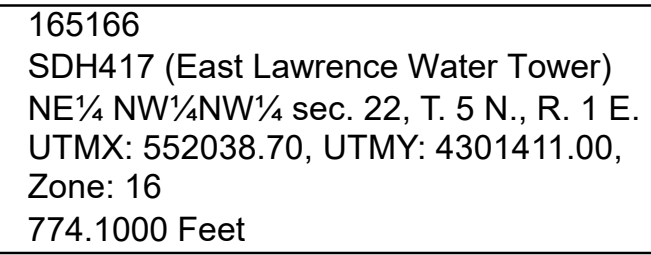 & 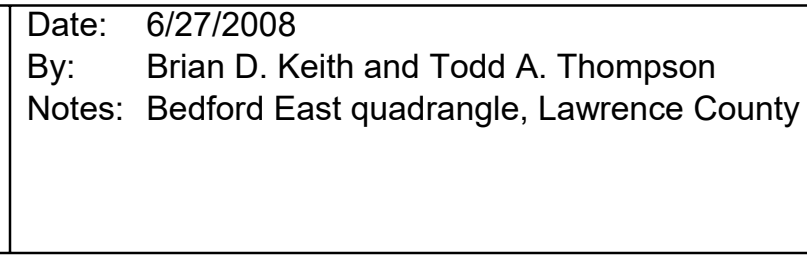 \\
\hline
\end{tabular}

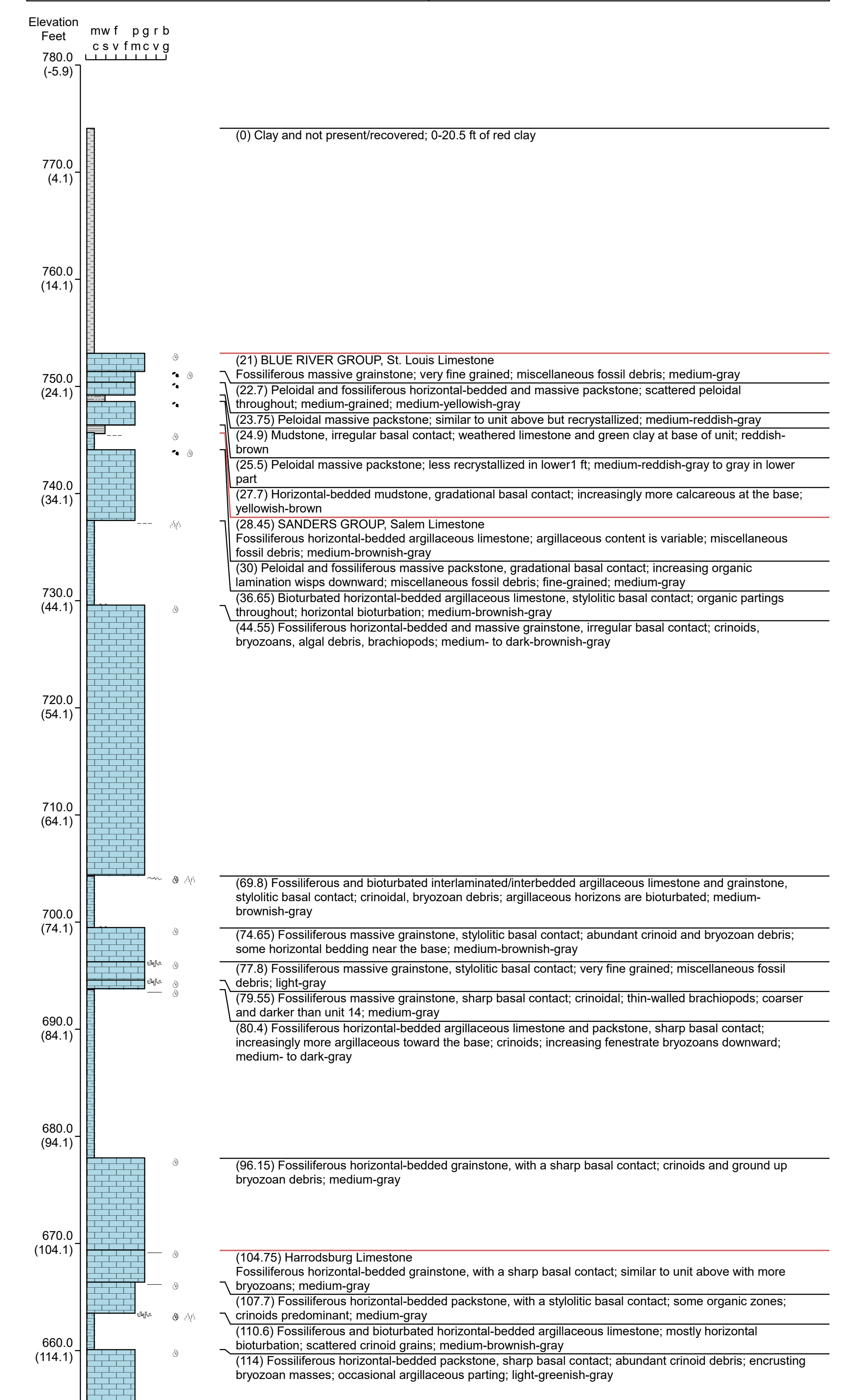

$\underset{(124.1)}{650.0}$

cha.

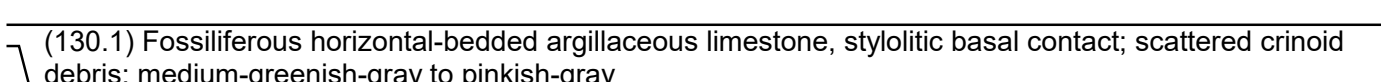

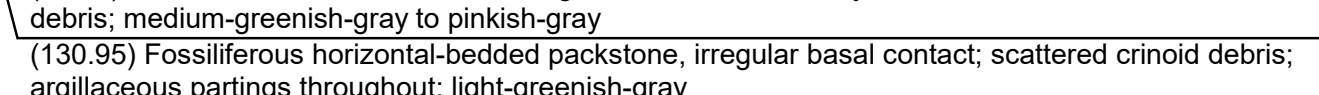

$\begin{array}{r}630.0 \\ (144.1) \\ \hline\end{array}$

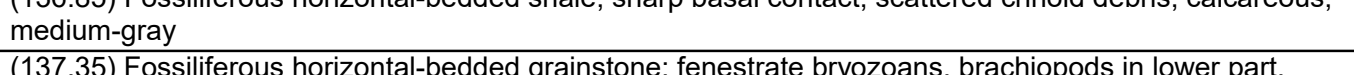

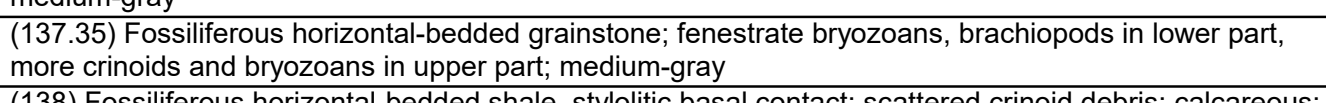

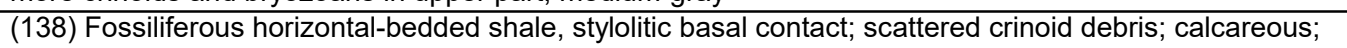

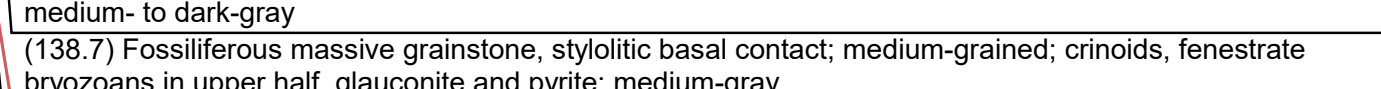

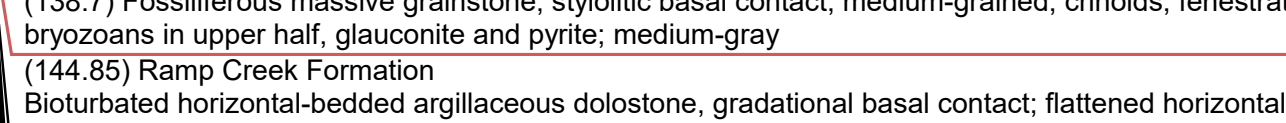

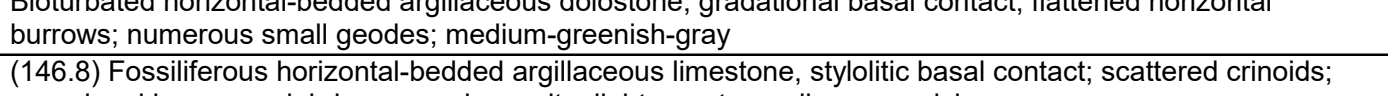

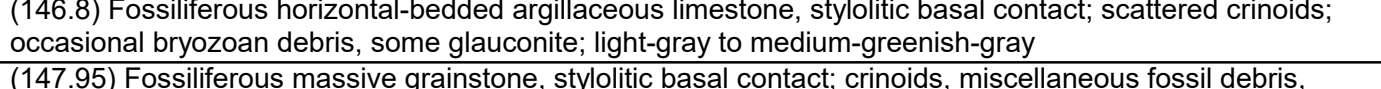

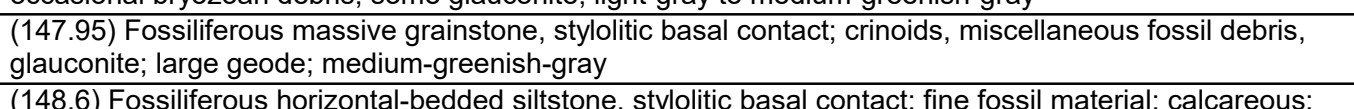

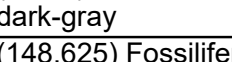

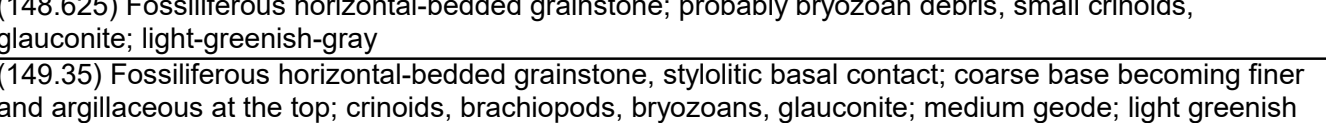

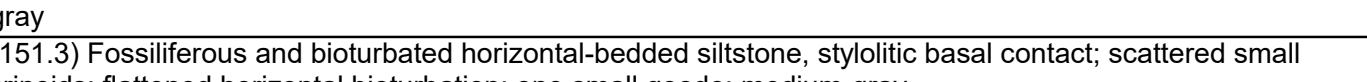

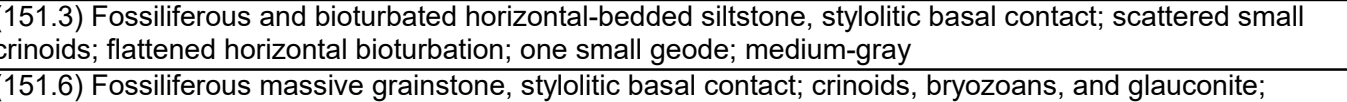
Medium-greenish-gray
(152:35)

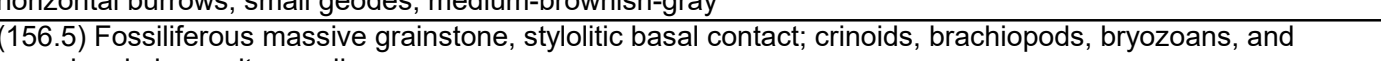

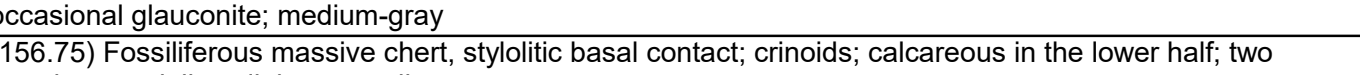

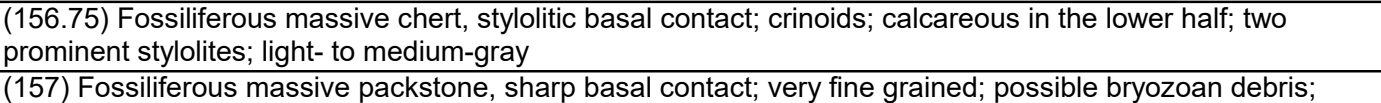

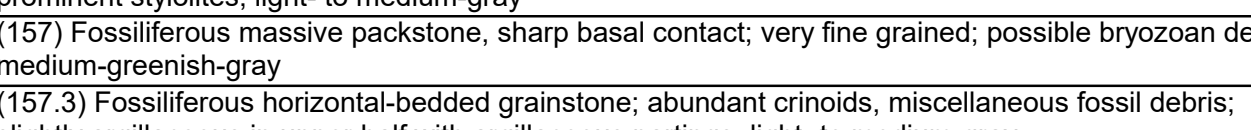

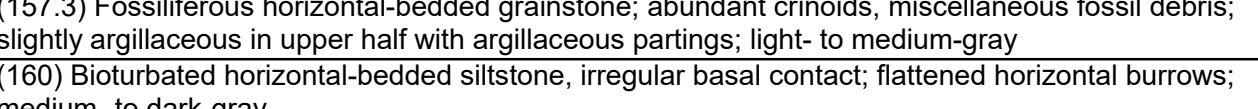

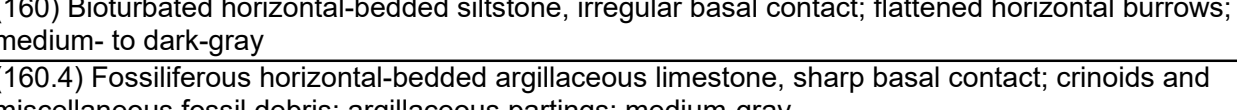

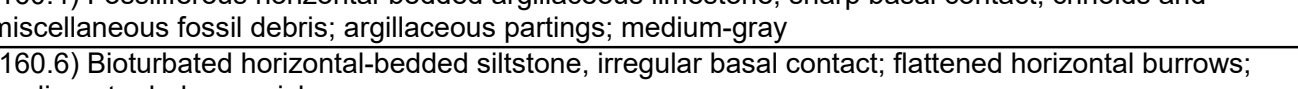

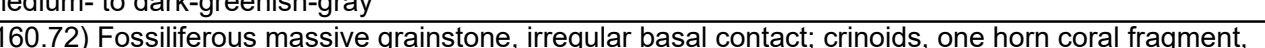

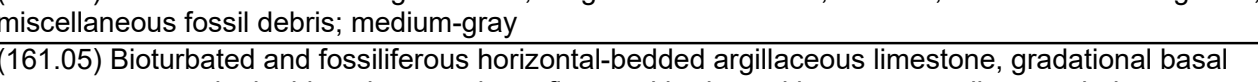

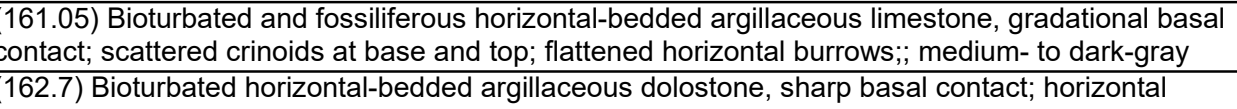

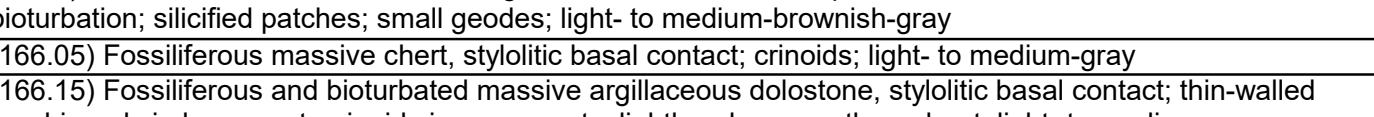

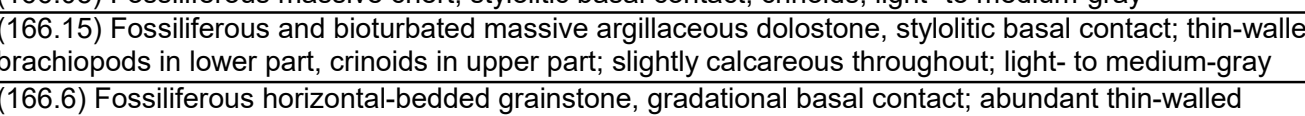

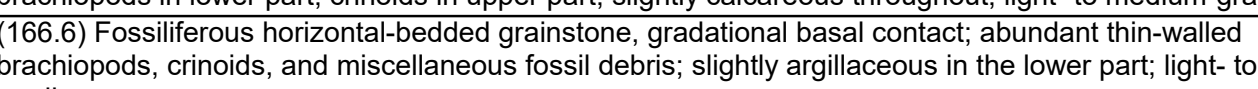

medium-gray
166.85) Bioturbated horizontal-bedded argillaceous dolostone, sharp basal contact; flattened horizontal

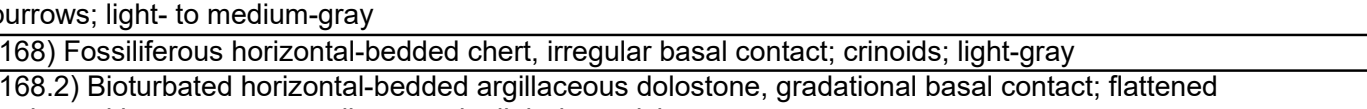

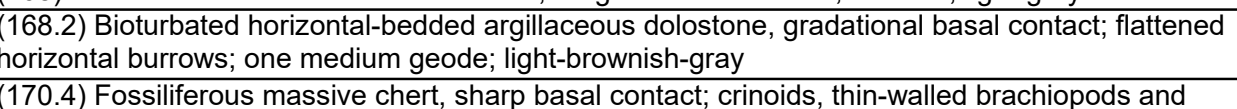

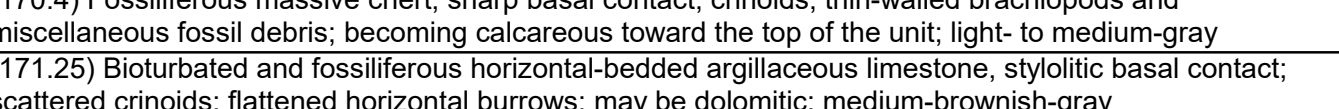

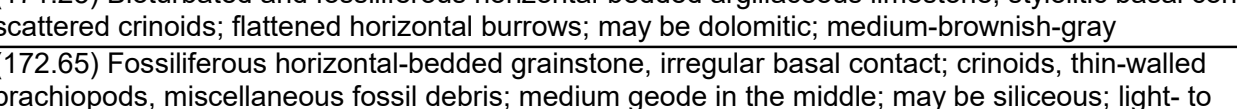

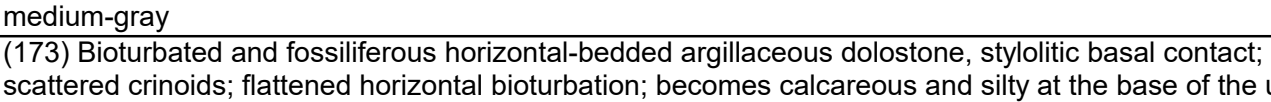

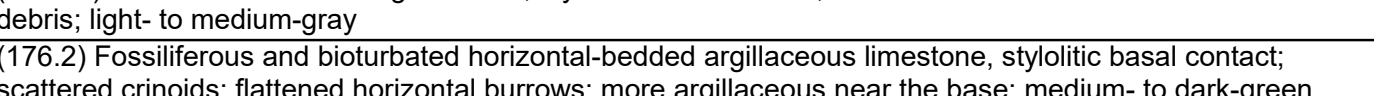
176.65) Bioturbated horizontal-bedded argillaceours dolostone; flattened horizontal burrous with vertical

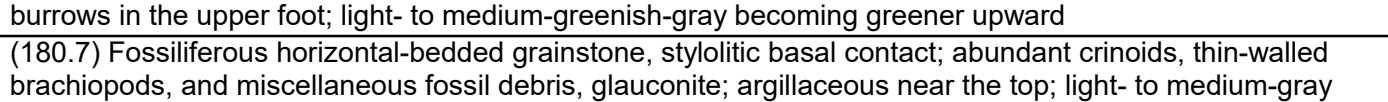

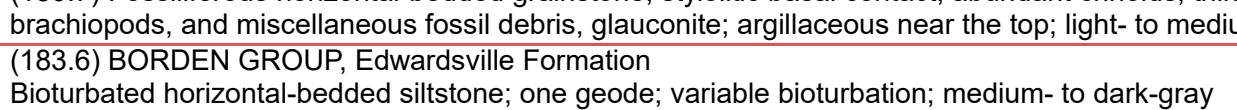




\begin{tabular}{|c|c|c|}
\hline $\begin{array}{l}\text { ID Number: } \\
\text { Site vam: } \\
\text { Location: }\end{array}$ & 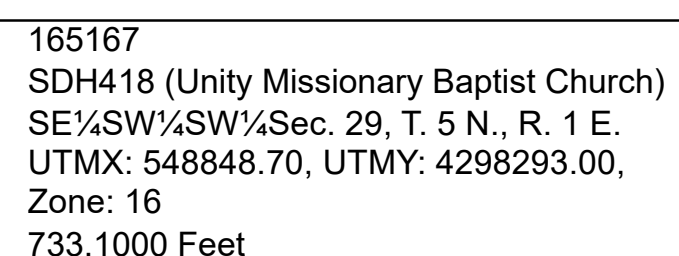 & $\begin{array}{l}\text { Date: } 6130120008 \\
\text { By: Todd A. Thompson and Brian D. Keith } \\
\text { Notes: Bedtord East quadrangle, Lawrence County }\end{array}$ \\
\hline
\end{tabular}

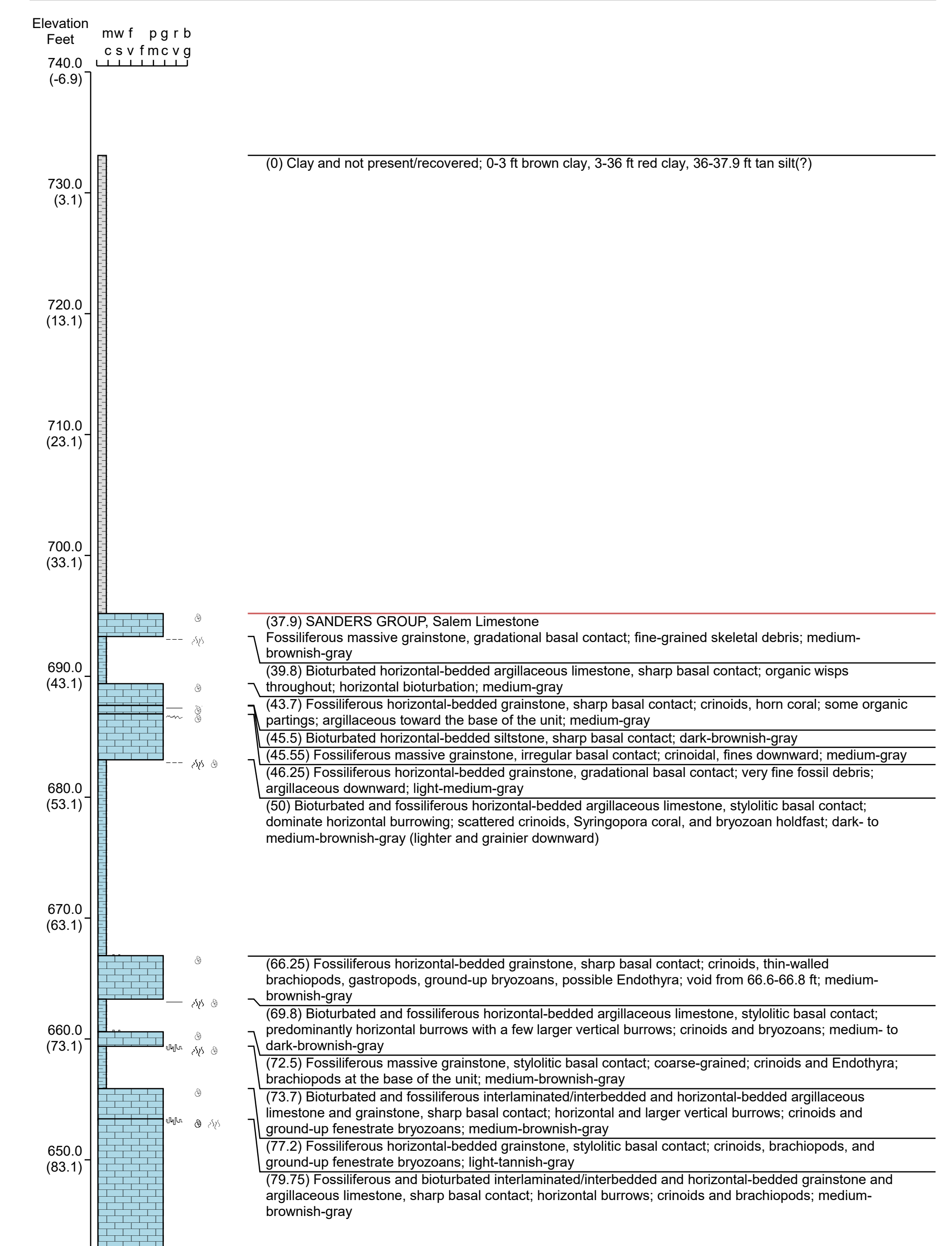

640.0
$(93.1)$
$(19$

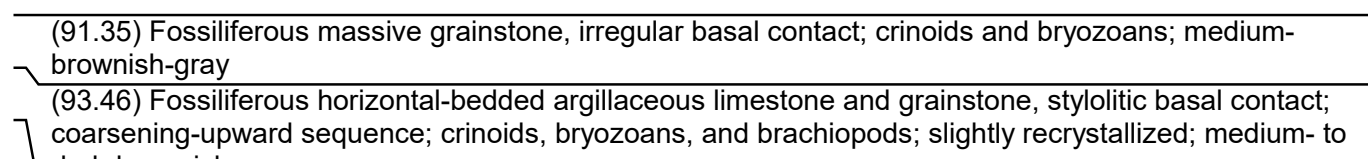

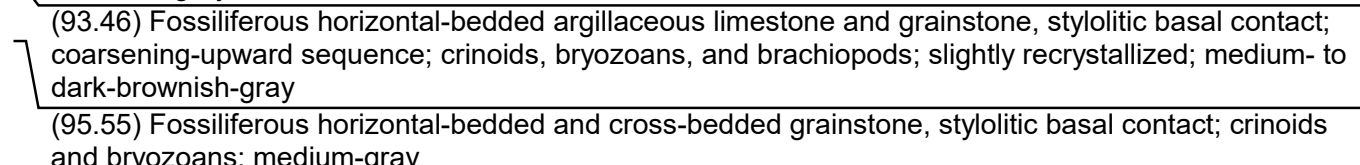

\begin{tabular}{c}
630.0 \\
$(103.1)$ \\
\hline
\end{tabular}

and broczans; medumm-gray

620.0
$(113.1)$

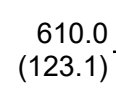

- =

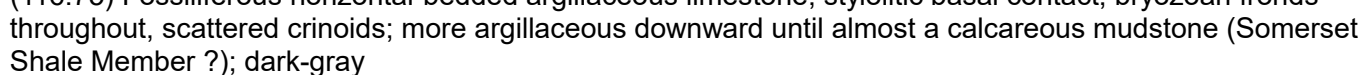
Shale Member ?; dark-gray

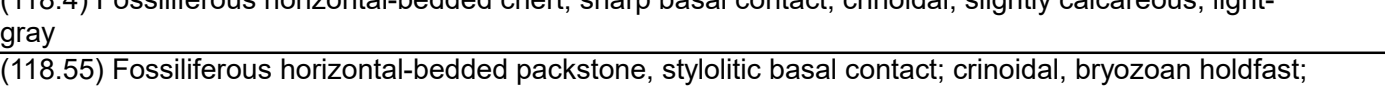

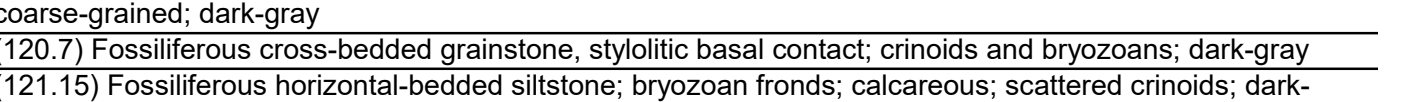

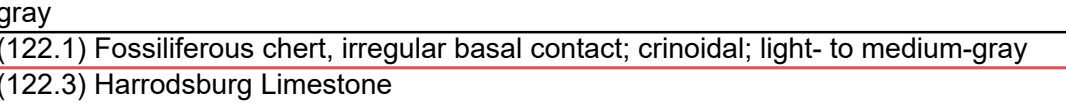
Fositiferous cross-bedded and horizontal-bedded grainstone, Styllitic basal contact, crinoids,

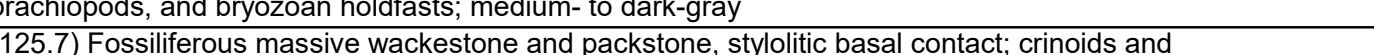

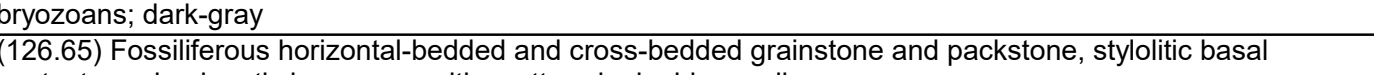

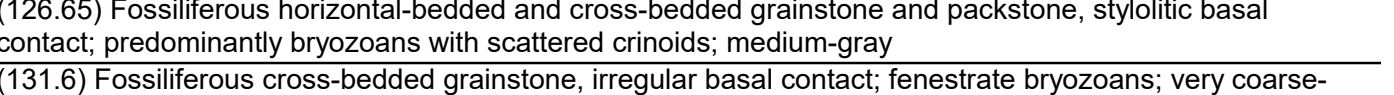

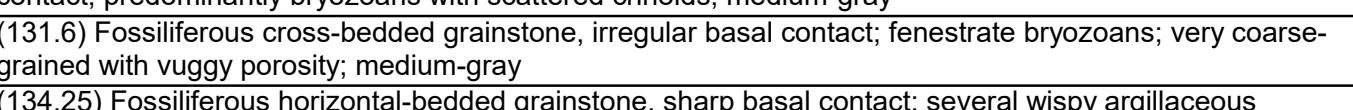

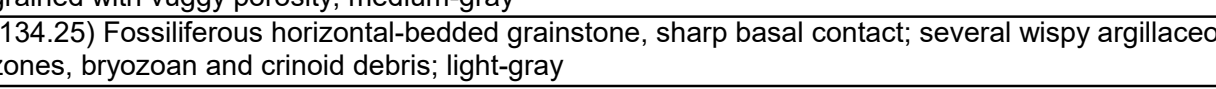

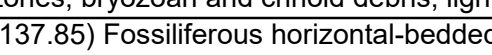

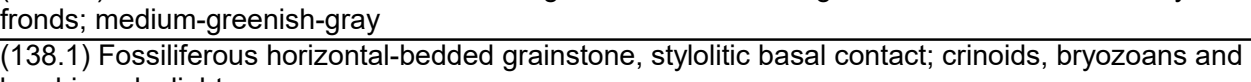

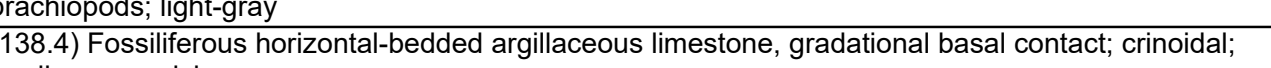

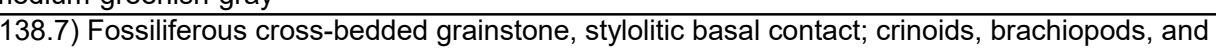

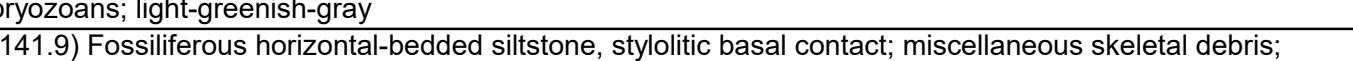

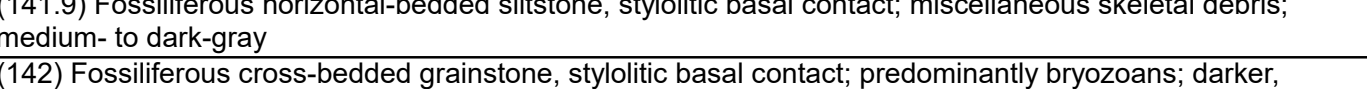

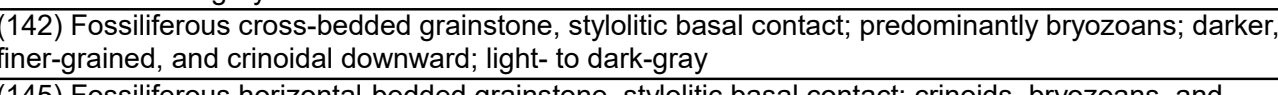

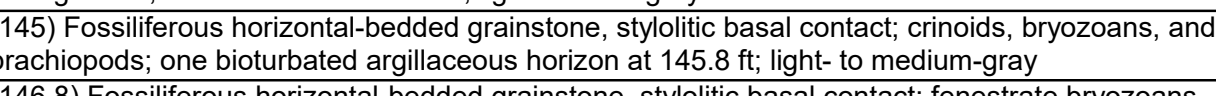

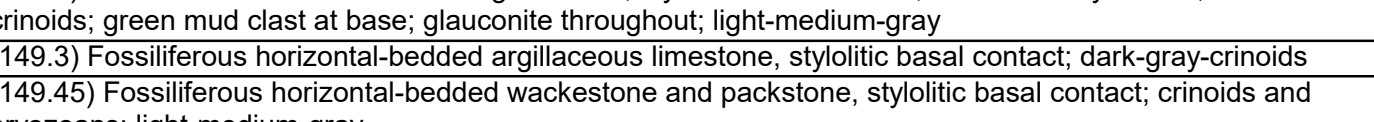

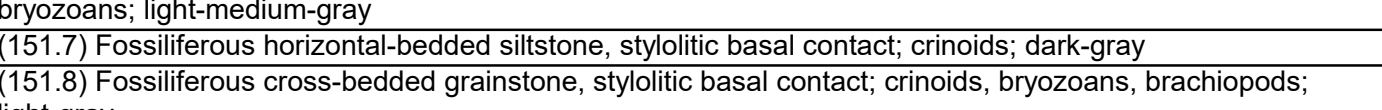

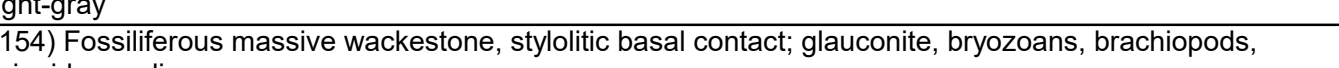

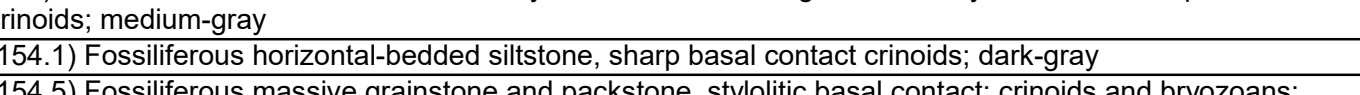

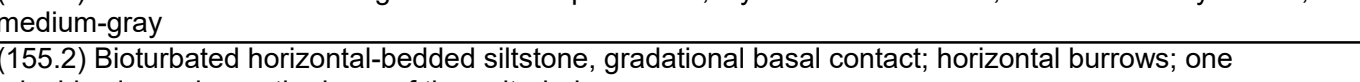

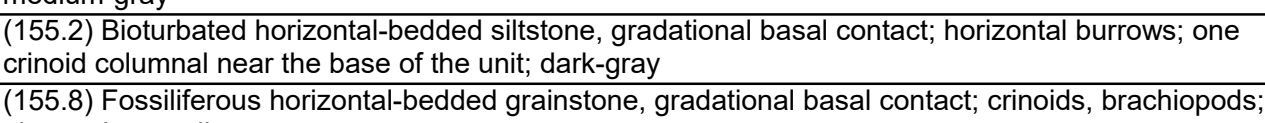

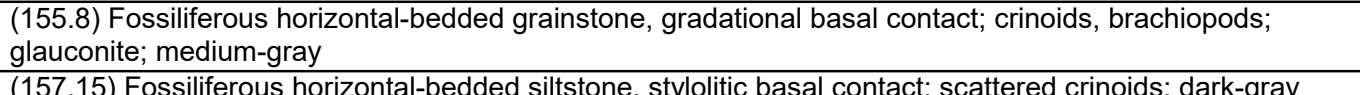

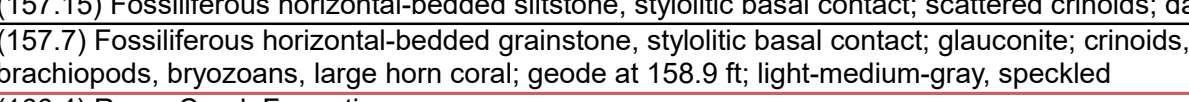

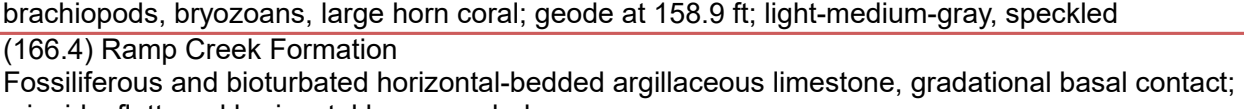

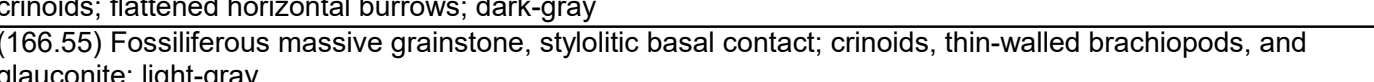

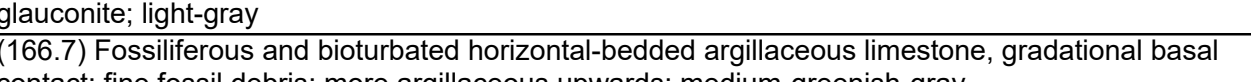

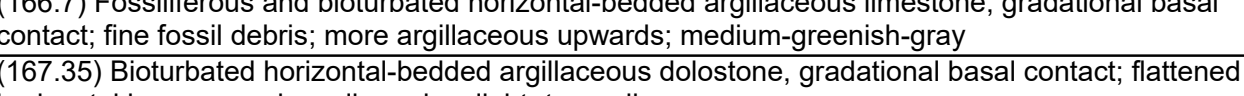

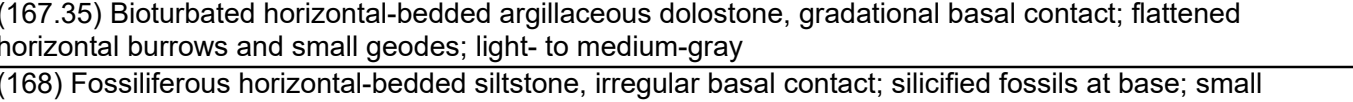

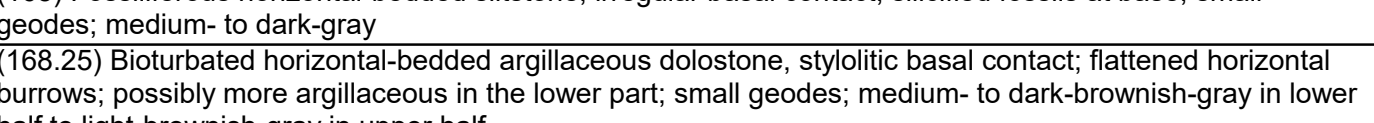

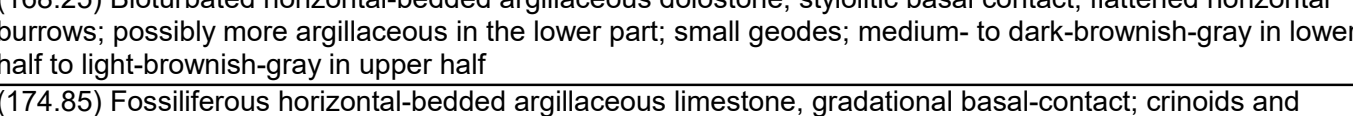

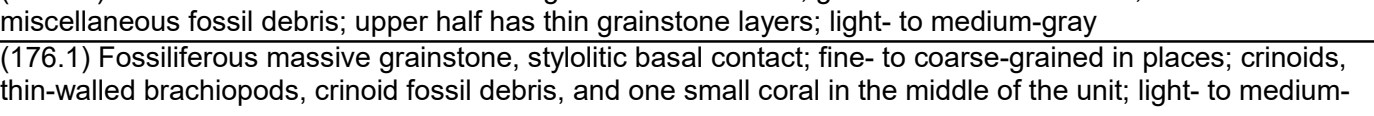

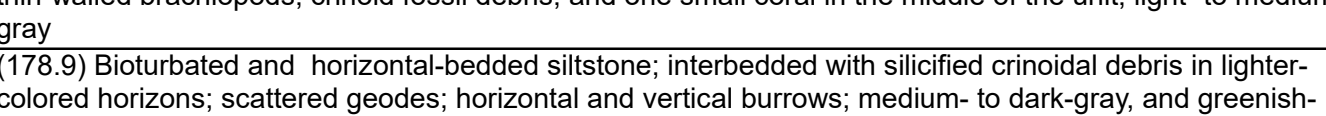

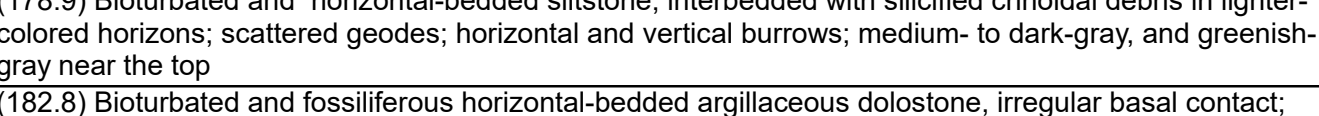

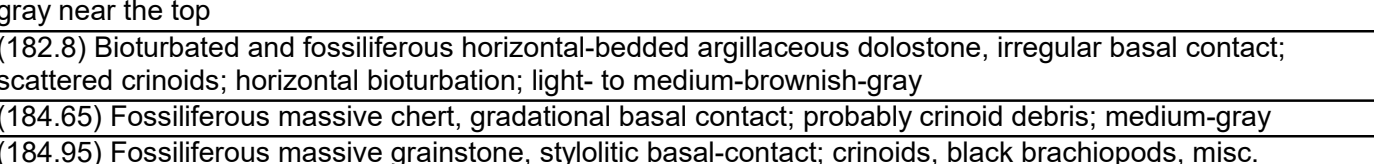

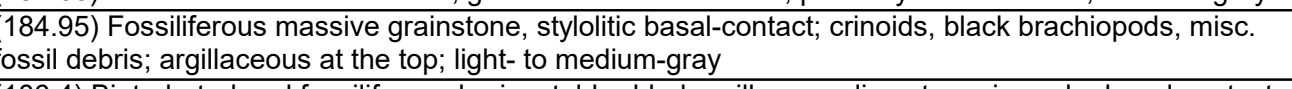

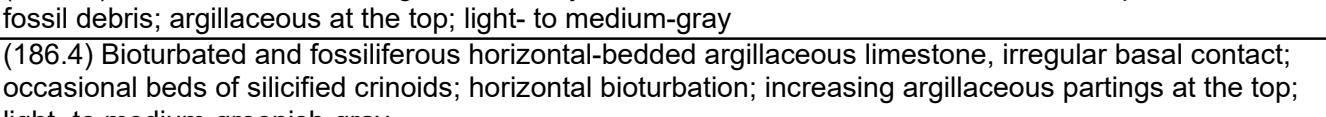

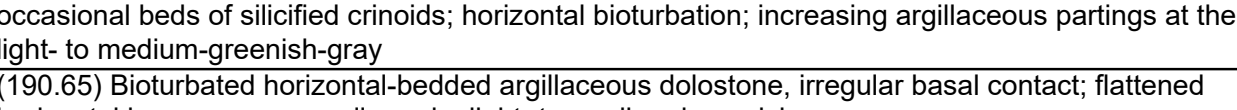

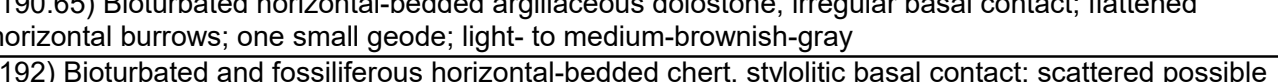

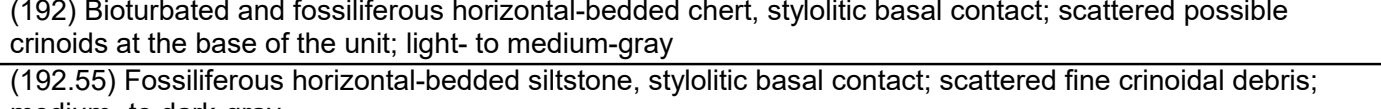

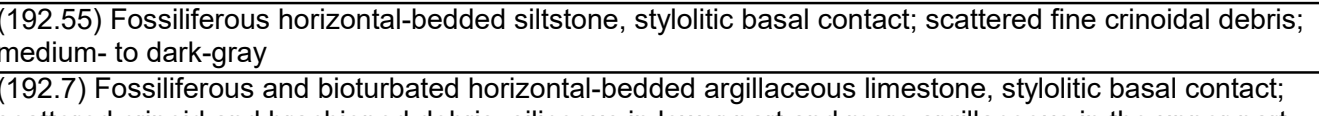




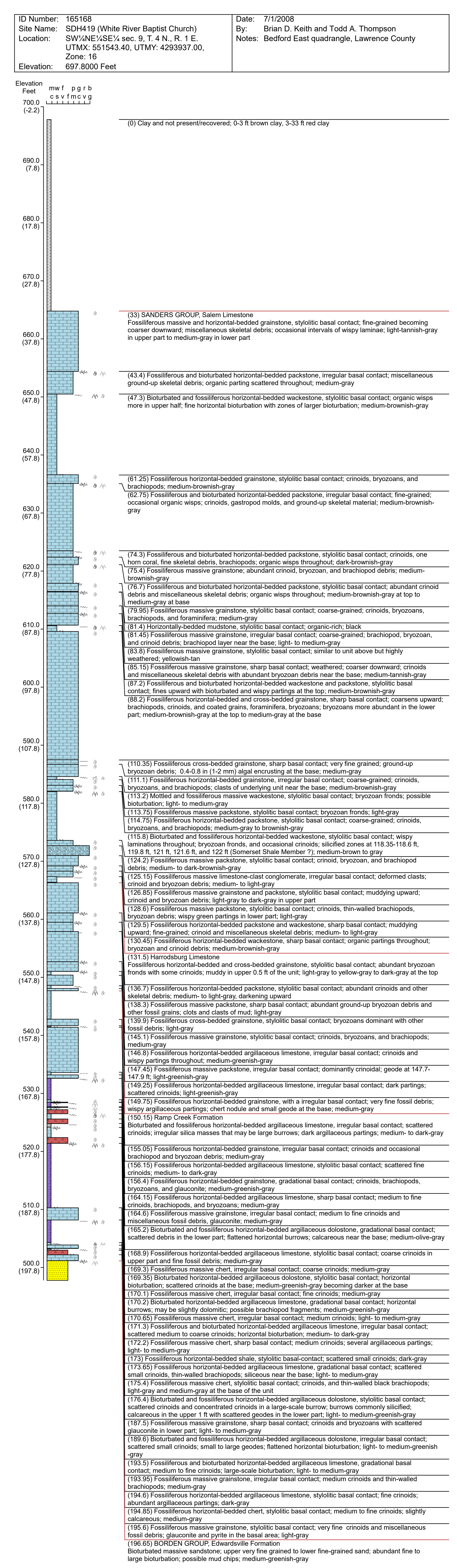




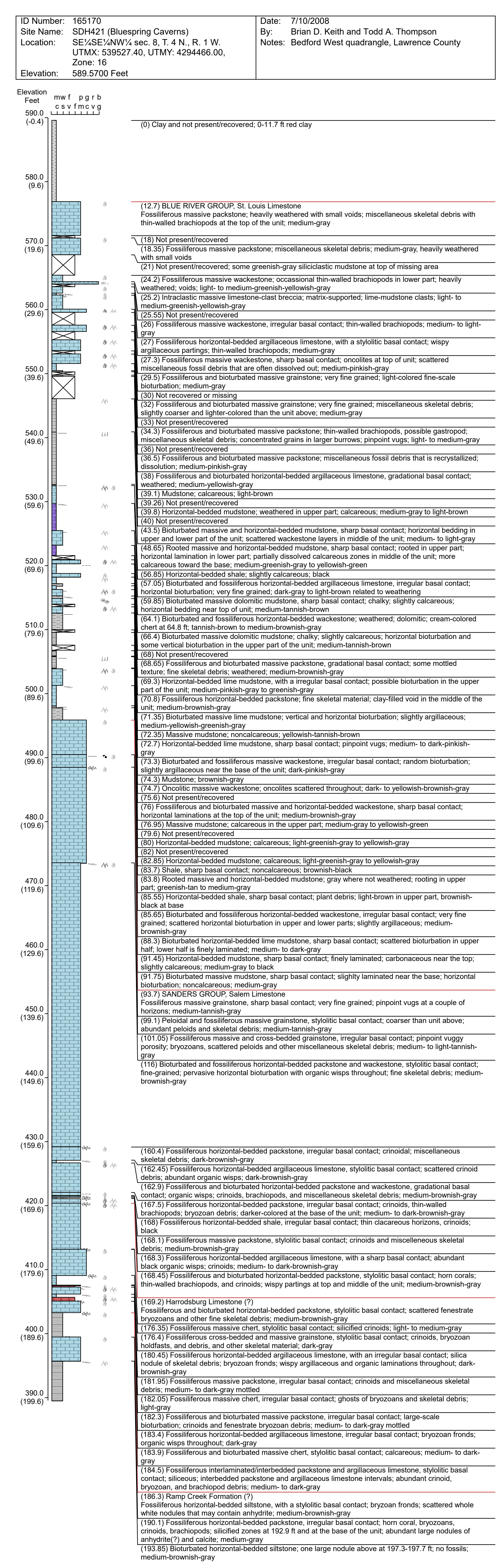




\begin{tabular}{|c|c|c|}
\hline \begin{tabular}{|l} 
ID Number: \\
Sitit Name: \\
Location:
\end{tabular} & 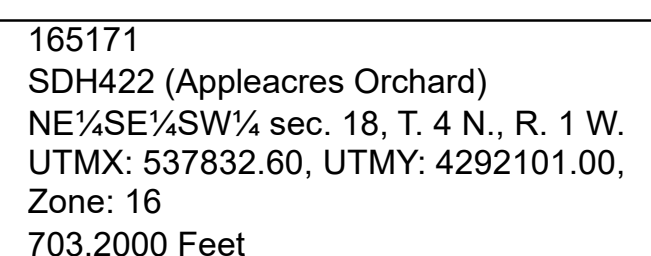 & 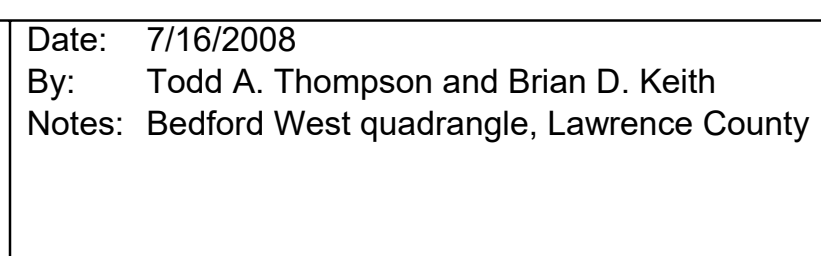 \\
\hline
\end{tabular}

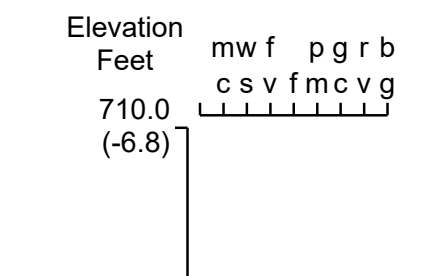

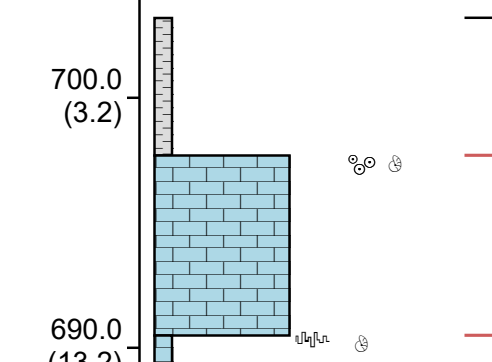
(0) Clay and not presentrecovered; sandy clay

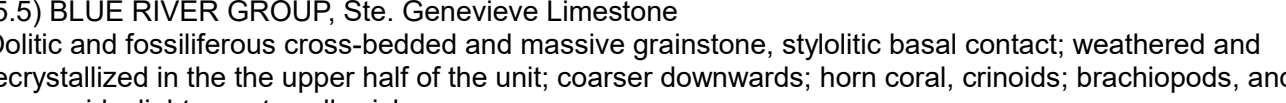

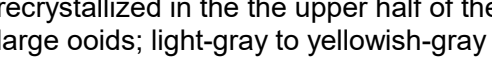

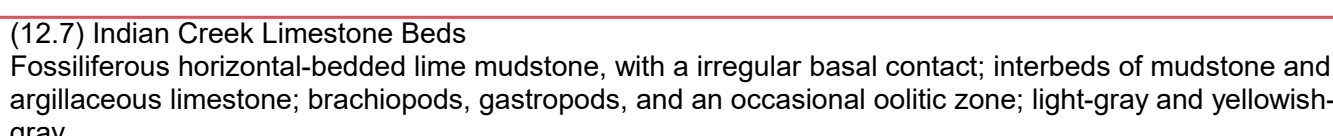

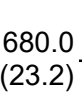
670.0
$33.2)$

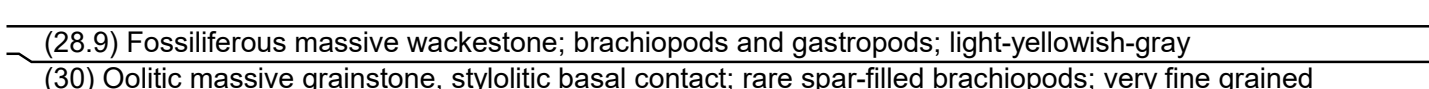 - ofites: ight-5ay)

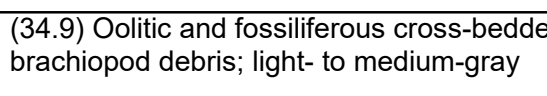 38.7) Massive lime mudstone, sharp basal contact; some fine argillaceous wisps throughoutt medium- $\frac{\text { gray }}{(41.8) \text { Oolitic and fossiliferous massive grainstone, stylolitic basal contact, gastropods and brachiopods; }}$

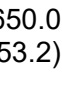
$(60.2)$
30.0
$(33.2)$
$(3)$
${ }_{(83.2)}^{620.0}$
610.0
$(93.2)$

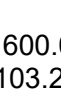
$\begin{array}{r}590.0 \\ (113.2) \\ \hline\end{array}$
580.0
$\begin{array}{r}570.0 \\ (133.2) \\ \hline\end{array}$
560.0

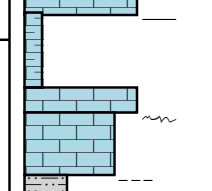
(54.7) Fossiliferous horizontal-bedded wackestone, sharp basal contact; some argillaceous wisps:

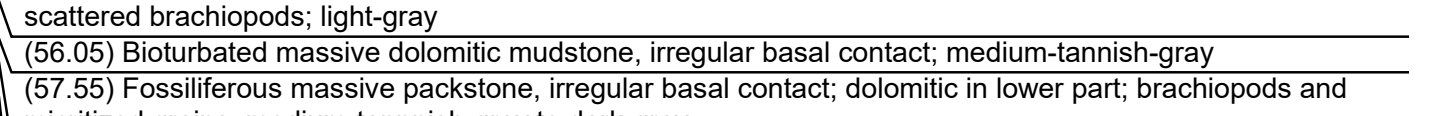

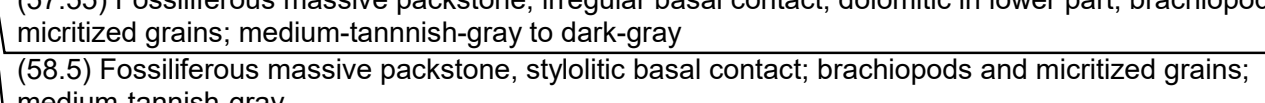
nedium-tannish-gray
$59.9)$ Fossiliferous massive grainstone, irregular basal contact; brachiopods and other skeletal debris;

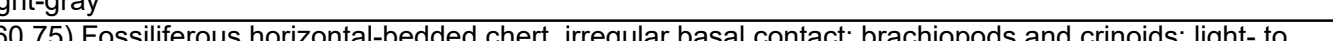
\begin{tabular}{l} 
medium-gray \\
\hline 61$)$ Fosiliferous massive packstone, irregular basal contact; brachiopods and other skeletal debris; ilight-
\end{tabular}
lannish-gray 16 .45) Fosiliferous horizontal-bedded chert, irregular basal contact, brachiopods and crinoids; i ight- to
nedium-gray
$62.2)$ Fossiliferous and oollitic horizontal-bedded grainstone, gradational basal contact, very fine skeletal
debris; coarre brachiopods at
(76.6) SC. Louis Limestone
Fossiliferous horizontal-bedde
ossiliferous horizontal-bedded packstone; argillaceous wisps throughout; miscellaneous skeletal debris;
medium-gray
$(80.2)$ Fossilfierous massive packstone, styllitici basal contact; brachiopods and other skeletal debris; medium-gray
(82.5) Massive dolostone, sharp basal contact; bioturbated at top of unit; scattered small calcite crystals; dark-yellowish-gray
$\begin{aligned} & \text { (85) Fossifiterous massive grainstone, styolitic basal contact; brachiopods and other skeletal debris; } \\ & \text { medium-raray }\end{aligned}$
(92.45) Massive dolostone, irregular basal contact; medium-yellowish-gray
(94.5) Intraclastic massive dolostone-clast breccia, irregular basal contact; clast-supported; medium-
(94.9) Massive dolostone, gradational basal contact; recrystallized; medium-yellowish-gray
(97.4) Bioturbated horizontal-bedded lime muddstone, stylolitic basal contact; recrystallized; bioturbated

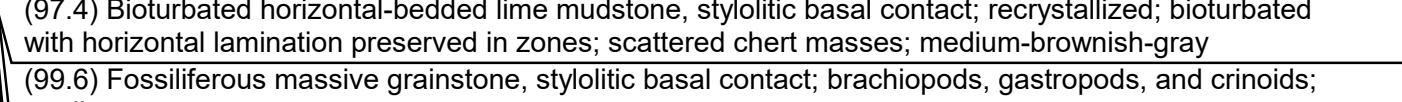 nedium-gray
99.75) Fossiliferous horizontal-bedded lime mudstone, styllititic basal contact, horizons of brachiopods

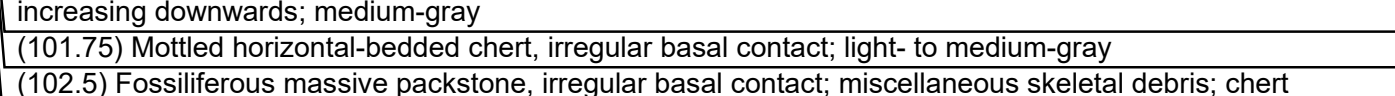
masses; medium- to light-gray
(105.2) Massive lime mudstone, sharp basal contact, light-medium-gray
(105.5) Fossiliferous horizontal-bedded chert, sharp basal contact; silifified crinoid debris; light- to
(105.6) Fossiliferous horizont
106.6) Bioturbatrated massive dolostone, styllitic basal contact, bioturbated and calcareous at top of unit;

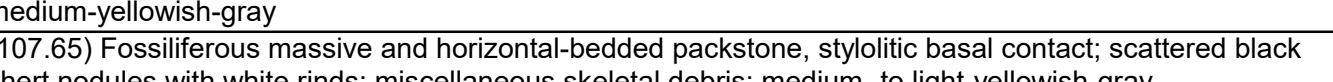
112) Fossiliferous horizontal-bedded wackestone, stylobitic basal contact; fenestrate bryozoan fronds,

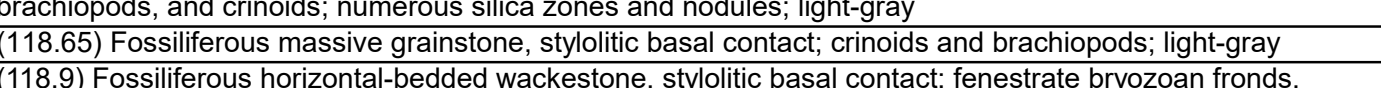 \begin{tabular}{l} 
brachiopods, and crinoids; scattered chert masses in upper part of unit, light-gray to light-yellowish-gray \\
\hline 128.8 Horizontal-bedded argillaceous limestone, gradational basal contact; thin crinoidal lag at base;
\end{tabular}
$\frac{\text { medium-greenish-gray }}{(129.9) \text { Horizontal-bedded limestone, stylolitic basal contact; scattered chert masses; argillaceous in }}$

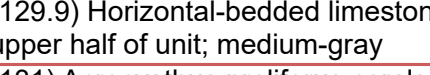
(131) Acrocyathus proliferus corals
Fossiliferous massive packstone, gradational basal contact; silicified finger corals; medium-gray
(132.85) Fossiliferous and bioturbated horizontal-bedded wackestone, styllolitic basal contact, fenestrate

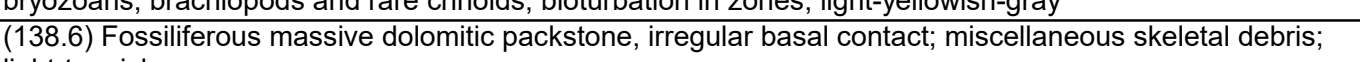
light-tannish-gray
(139.3) Fossilfirous horizontal-bedded wackestone, gradational basal contact; fenestrate bryozoans and

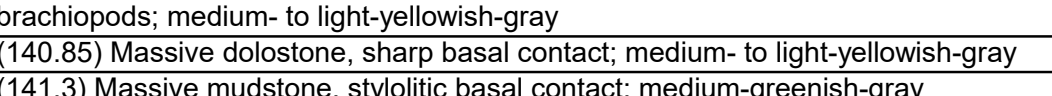

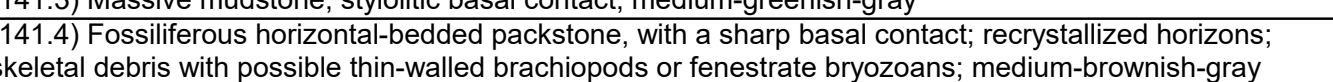

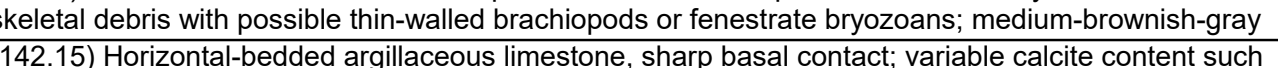
hat it is almost lime mudstone in places; greenish-gray
(145.15) Fossilfifrous horizontal-bedded packstone, irregular basal contact, coral and crinoids:

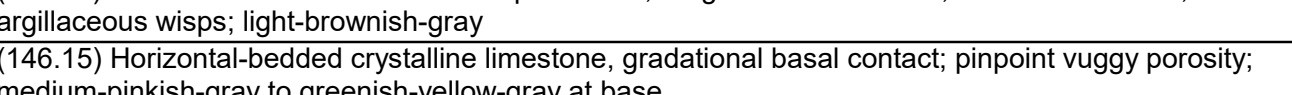
$\frac{\text { medium-pinkish-gray to greenish-yellow-gray at base }}{(148.6) \text { Mudstone; greenish-yellow }}$ 


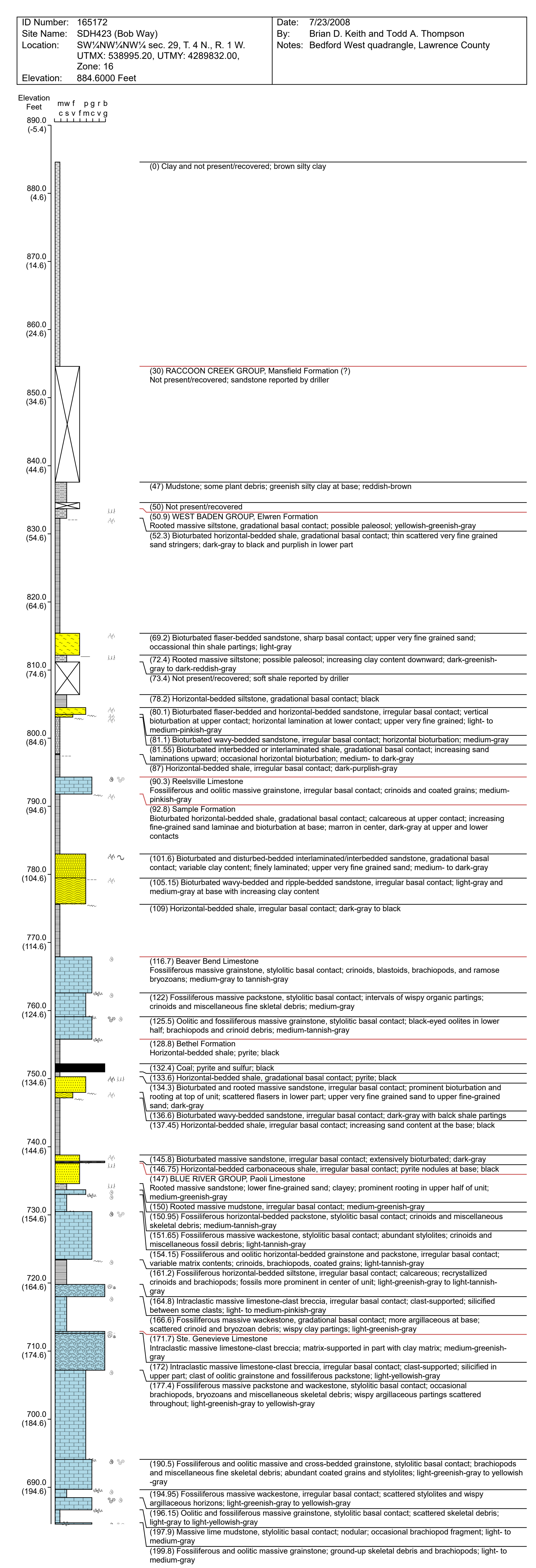




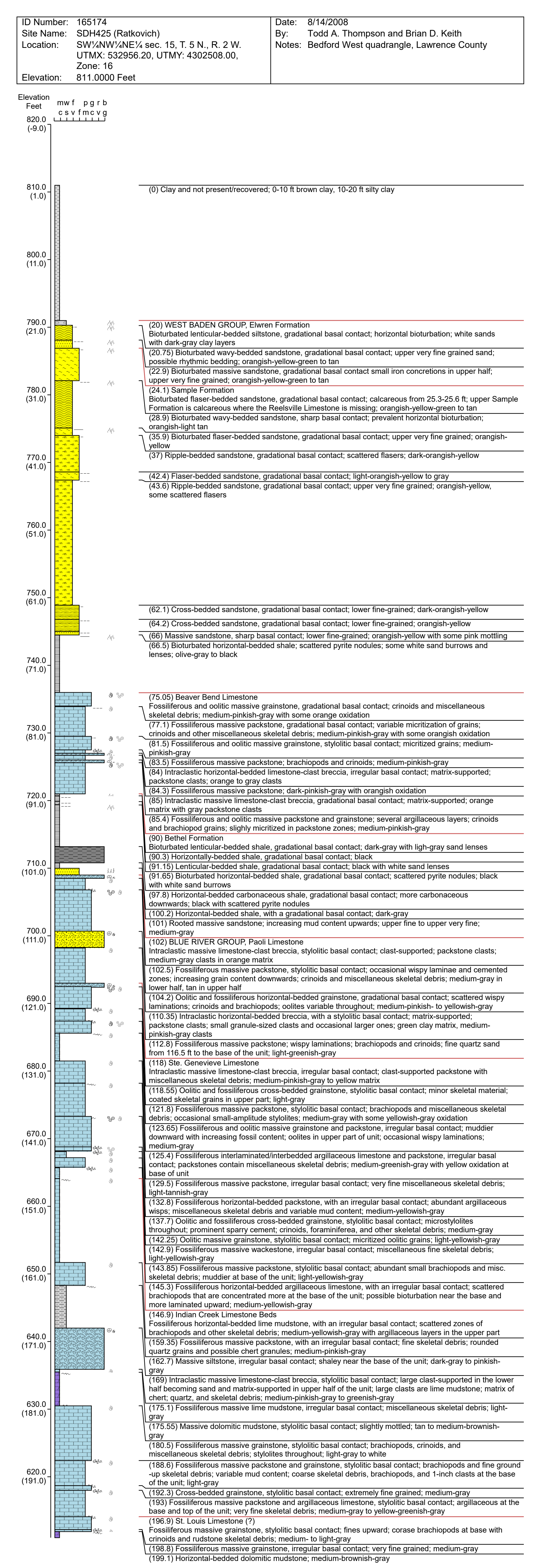




\begin{tabular}{|c|c|c|}
\hline \begin{tabular}{|l} 
IN N Number: \\
Site Name: \\
Location: \\
Elevation:
\end{tabular} & 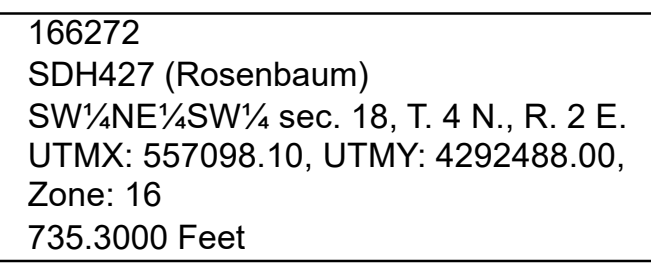 & 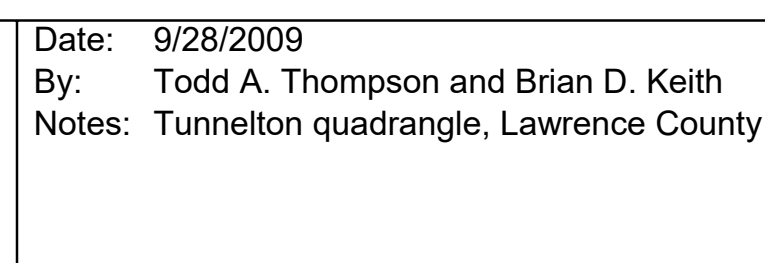 \\
\hline
\end{tabular}
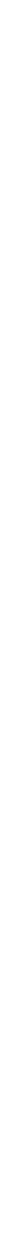

\begin{tabular}{c}
690.0 \\
$45.3)$ \\
\hline
\end{tabular}

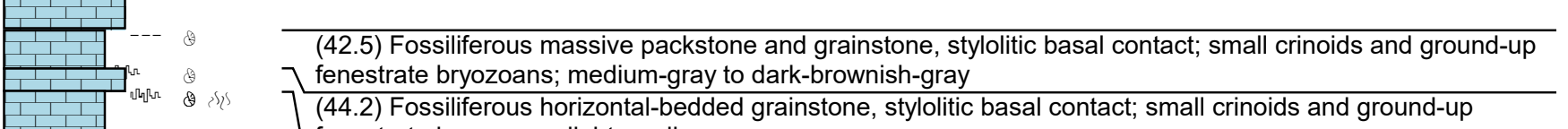
styllitic basal contact; small crinoids and ground-wp otturbated horizontal-bedded packstone, sharp basal contact; overall grainier

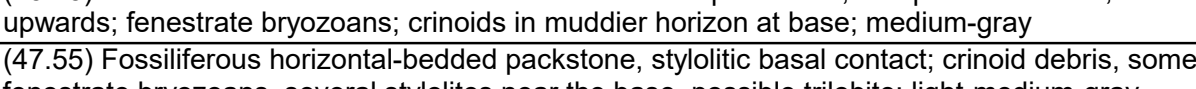
(48.75) Harrodsburg Limestone

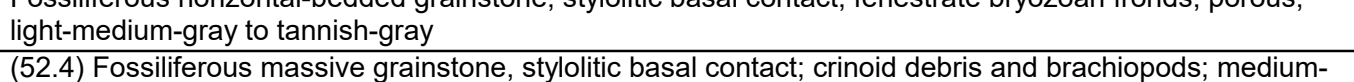
(52.9) Fossiliferous horizontal-bedded grainstone, stylolitic basal contact; crinoids, fenestrate bryozoans; LWo stylolites in the middle of the unit; medium-gray
(53.85) Fossilfifrous horizontala-beddded packstone, styolitic basal contact; crinoids, thin-walled

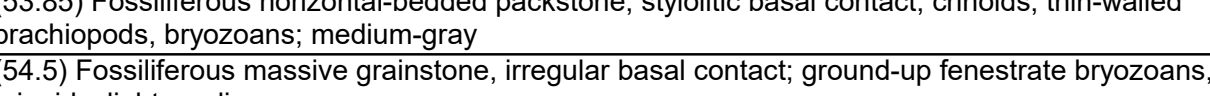
(55.5) Intraclastic and fossiliferous limestone-clast breccia, stylolitic basal contact; clast-supported; 年. small crinoids; medium-gray
$56.3)$ Fossilfifrous massive grainstone, stylolitic basal contact, ground-up fenestrate bryozoans, small

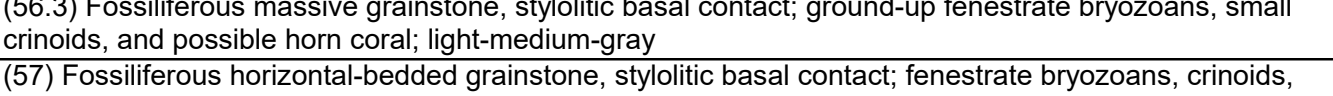

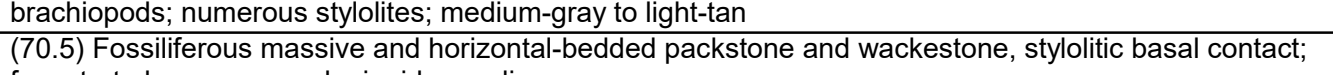

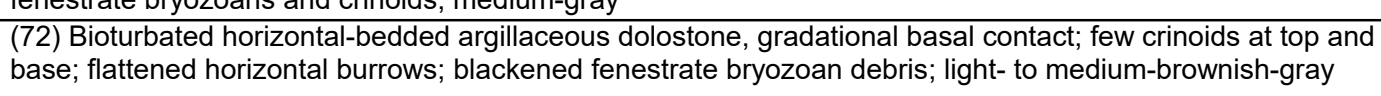
(74.4) Bioturbatede and fossiliferous horizontal-bedded argillaceous limestone, styllilitic basal contact;
scattered fine crinoids and fenestrate bryozoans, especially in the lower part; flattened horizontal bitturbation; dark-brownish-gray
(75.4. Fonssiliferous massive chert, irregular basal contact; medium to coarse crinoids, fenestrate bryozoans, and possible brachiopod debris, medium-gray
$75.6)$ Fossiliferous massive grainstone, gradational basal contact; medium to coarse crinoids, fenestrate bryozoans, and brachiopods; dark-gray
$75.85)$ Fossiliferous horizontal-bedded argillaceous limestone, styllilitic basal contact, fine crinoidal

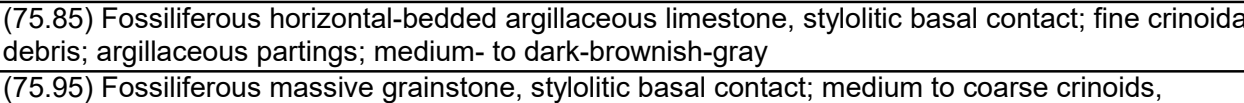

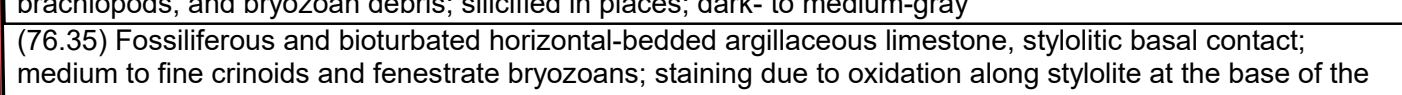

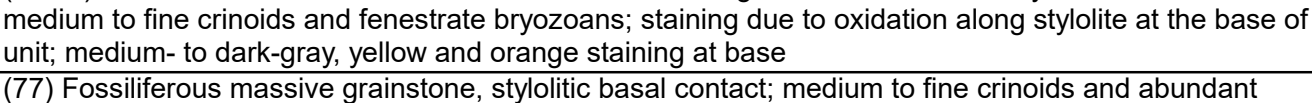

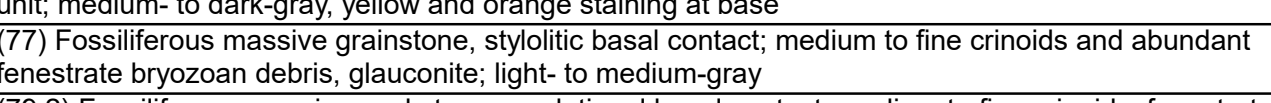

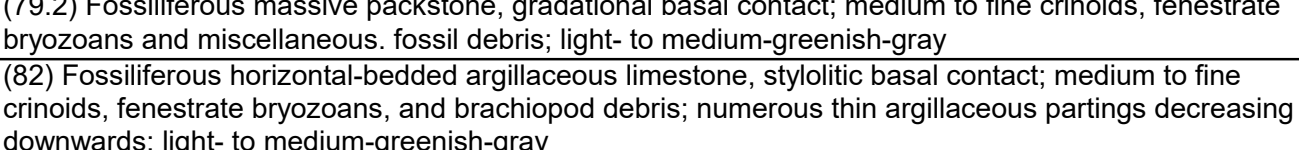

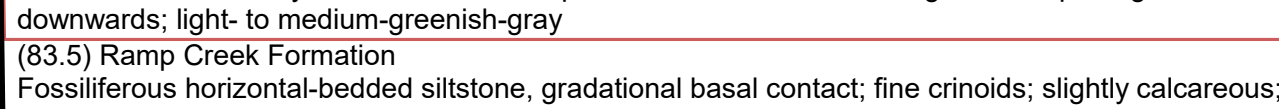
medium-grenilsi-gray
(83.95) Fossiliferous horizontal-bedded grainstone, gradational basal contact; fenestrate bryozoans and
then

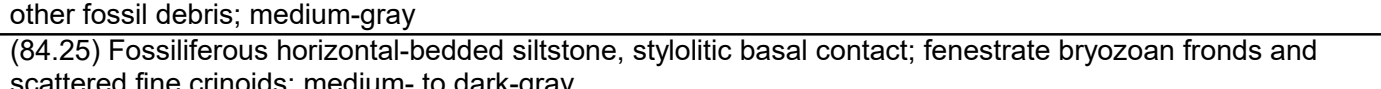

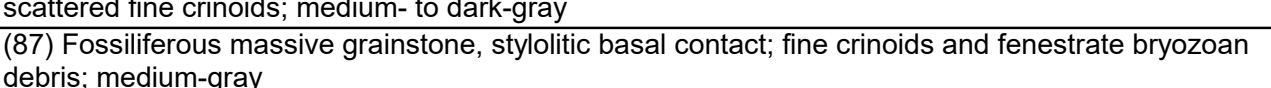

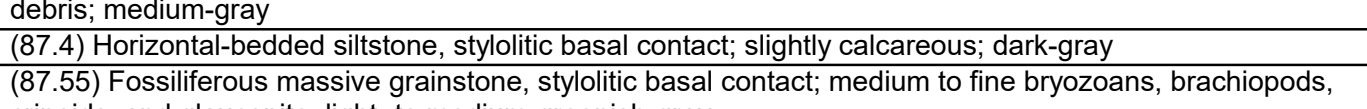

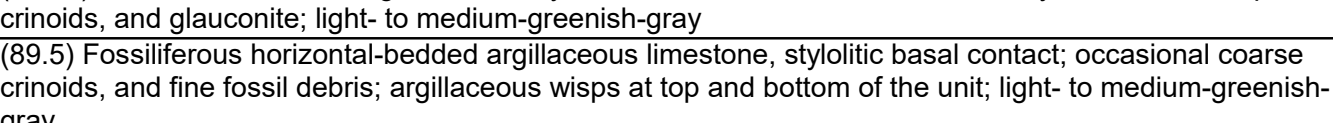
gray
9oo) Fossiliferous horizontal-bedded grainstone, stylliticic basal contact, fenestrate bryozoans, medium to
coarse crinoids and brachiopods, glauconite some fossils partially silicified light-gray

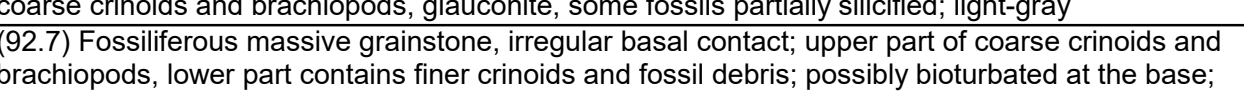
prominent styloliti in the middle of the unit, medium-gray
99.5)

9477) Fossiliferous horizontal-hedded argillaceous

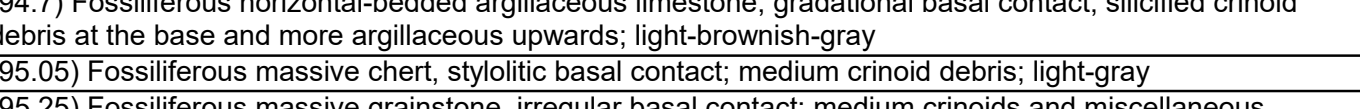

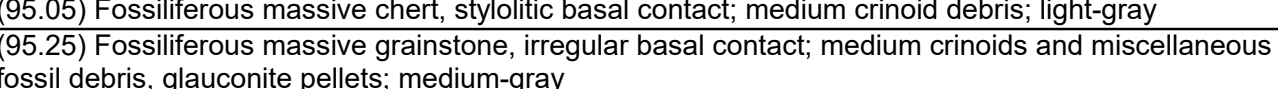

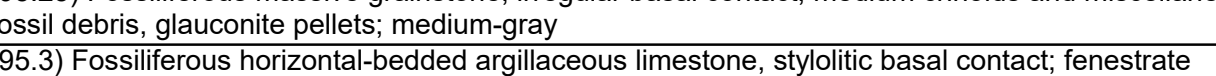

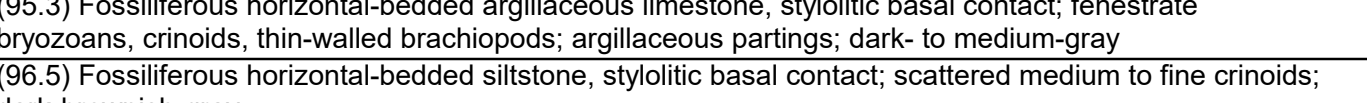
dark-brownish-gray
96.6)

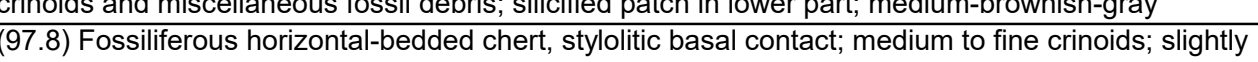

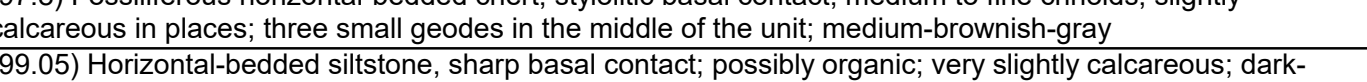

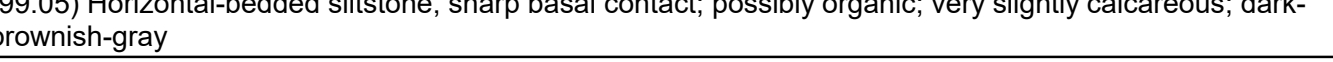

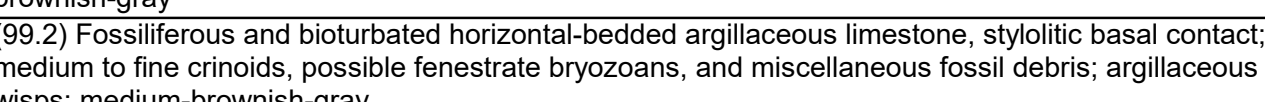

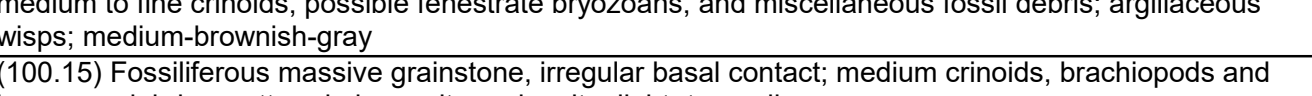

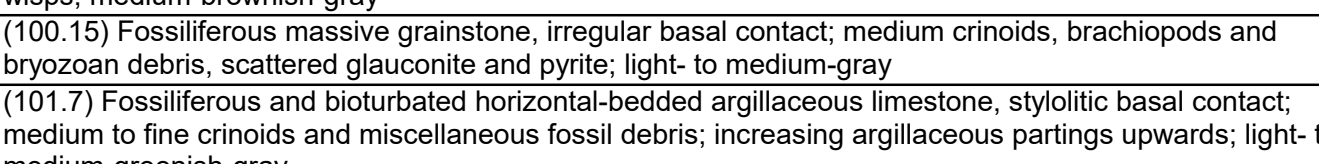

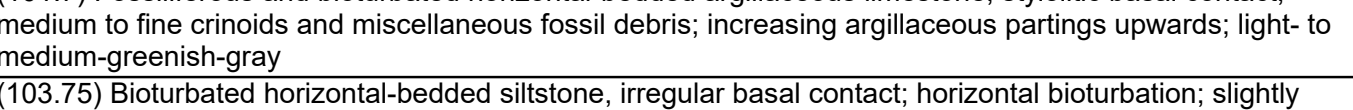

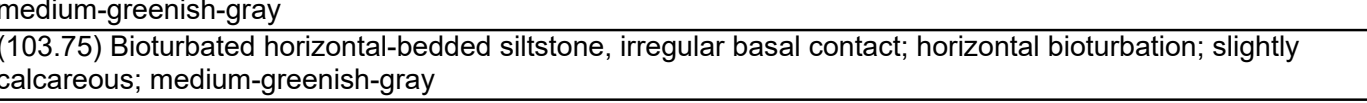

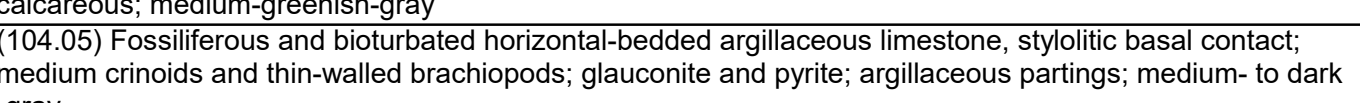
-gray
(105.6) Fossiliferous and bioturbated massive grainstone, stylliticic basal contact; coarse to medium

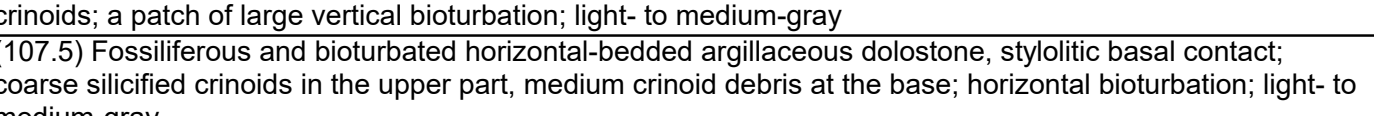
medium-gray
108.45 Fossiliferous massive chert, stylolitic basal contact, medium crinoids; medium-gray
108.6) Bioturbated horizontal-bedded argillaceous dolostone, stylolitic basal contact; one large crinoid segment near the top, scattered smal geodes,
brownish-gray

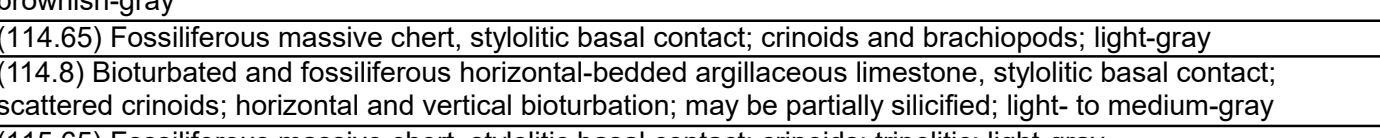

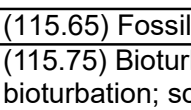
bioturbation; scattered small geodes; ligh-greenish-gray minstone beds; crinoids; i ight to dark-gray

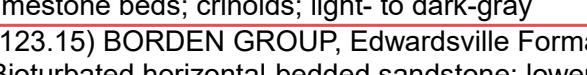

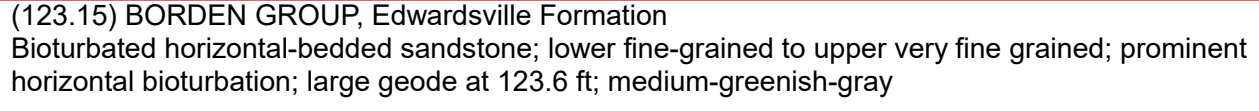




\begin{tabular}{|c|c|c|}
\hline \begin{tabular}{|l} 
IN N Number: \\
Site Name: \\
Location: \\
Elevation:
\end{tabular} & 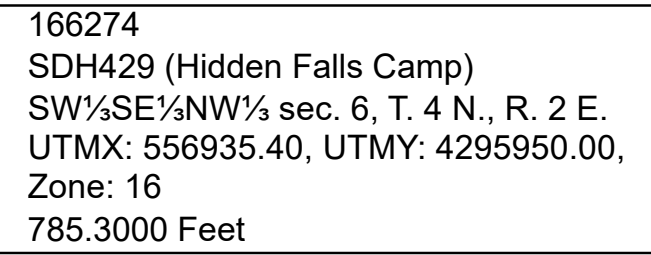 & $\begin{array}{l}\text { Date: } 9 / 292 / 2009 \\
\text { By: } \\
\text { Notes: : Trunnel.tith quadh adrangle, Lawrence County }\end{array}$ \\
\hline
\end{tabular}

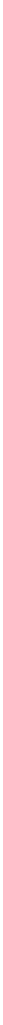

\begin{tabular}{c}
750.0 \\
$(35.3)$ \\
\hline
\end{tabular}

$7^{(30.2)}$ Fossiliferous horizontal-bedded grainstone, irregular basal contact; abundant crinoids and

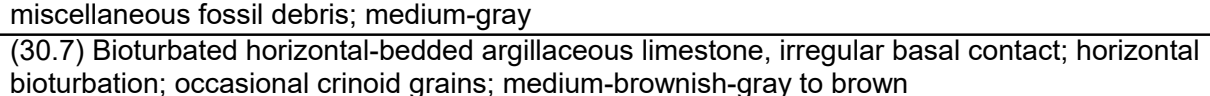

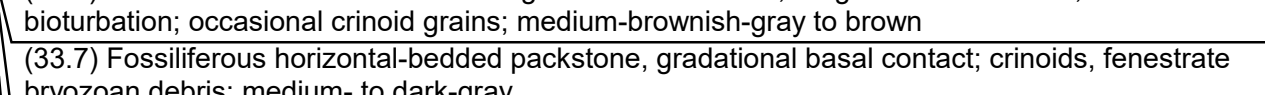

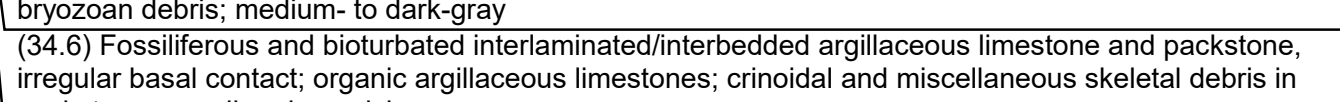

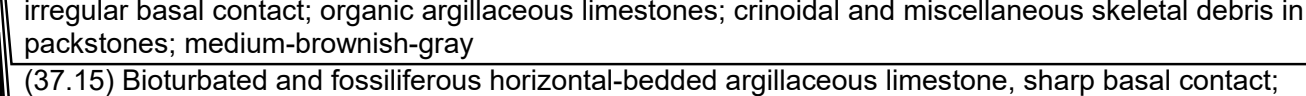

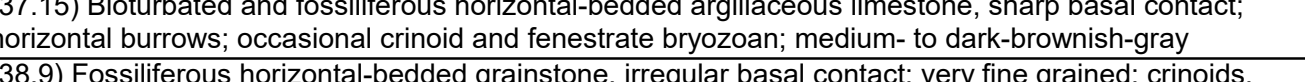

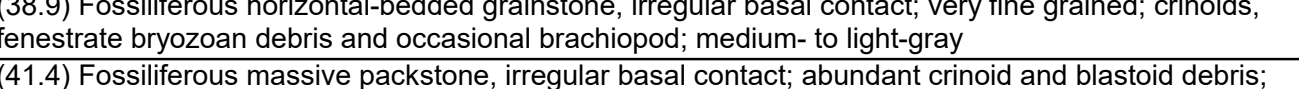

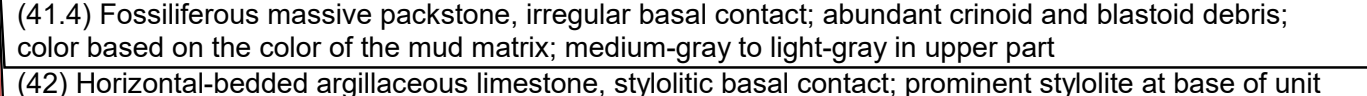

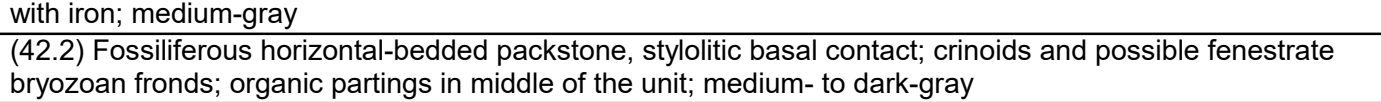

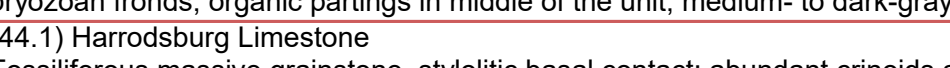

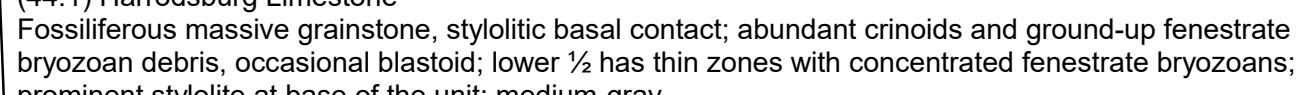

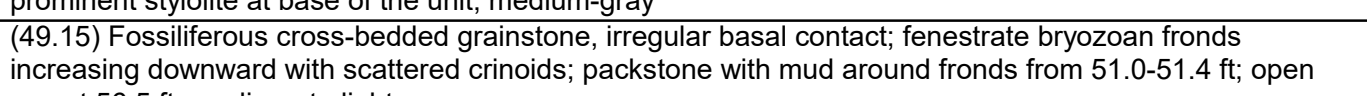

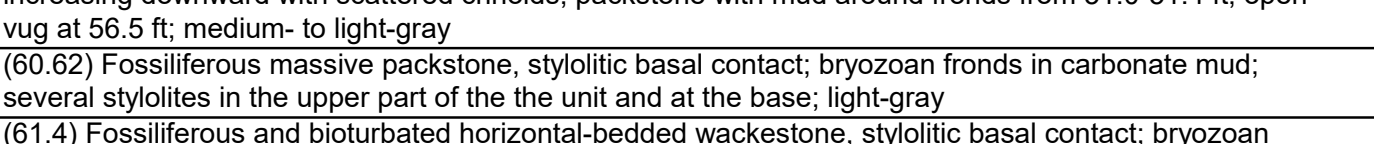

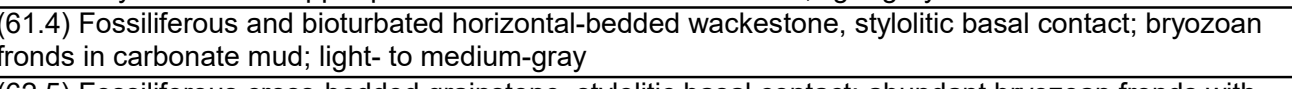

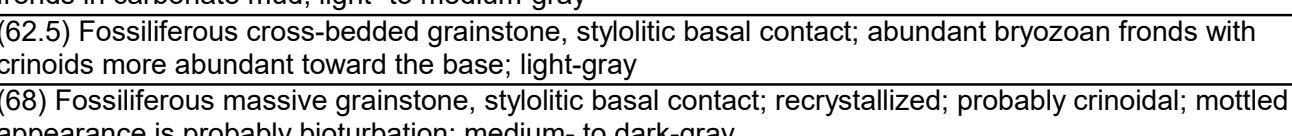

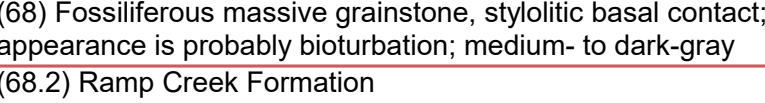

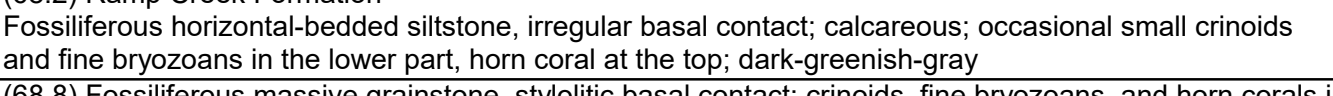

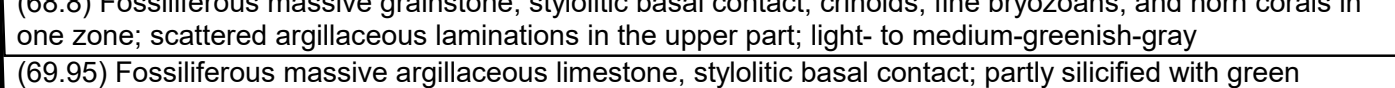

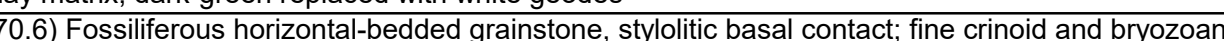

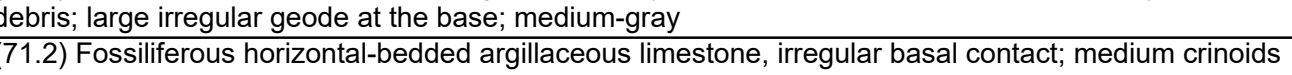

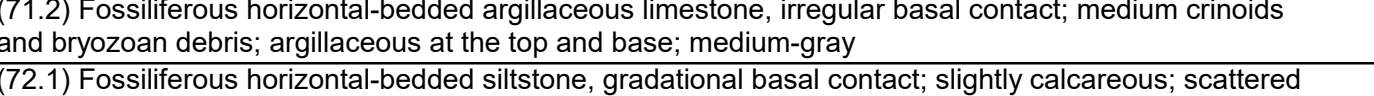

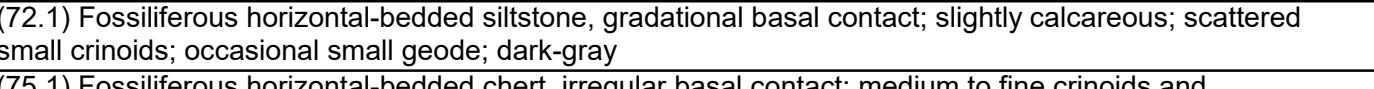

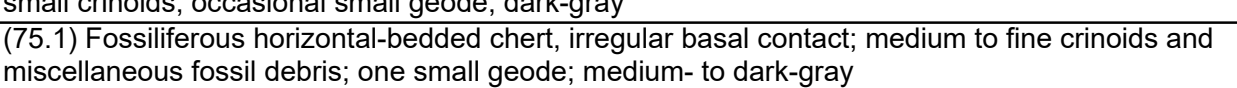

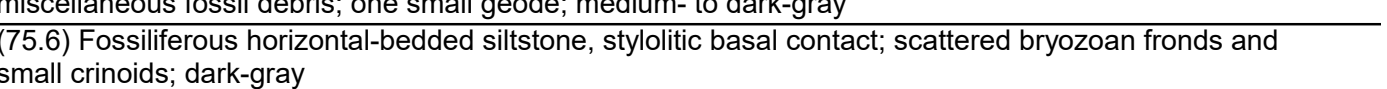

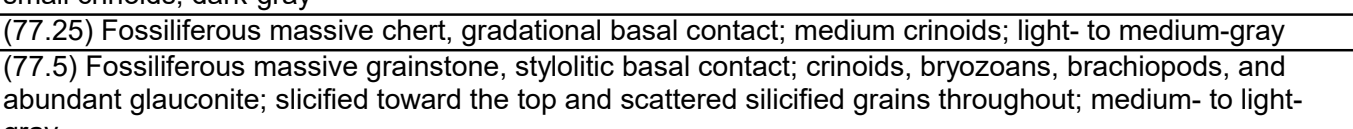

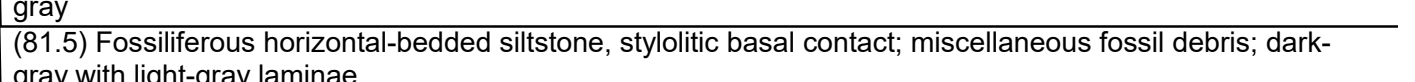

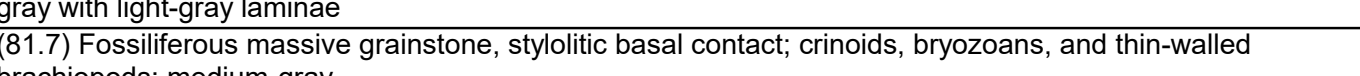

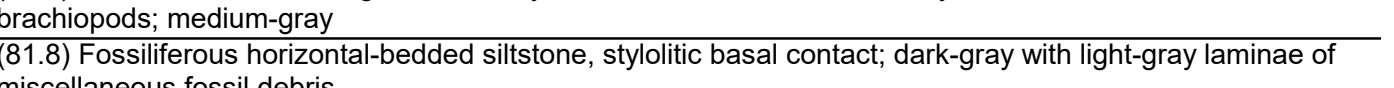

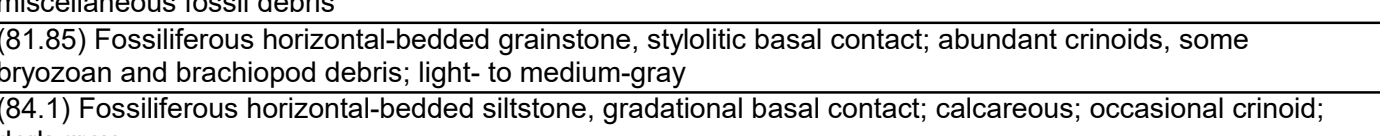

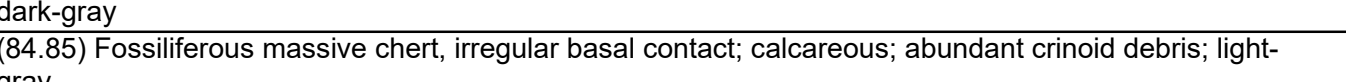

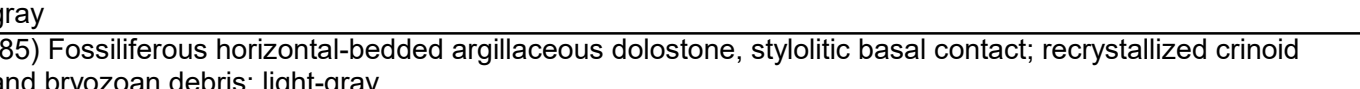

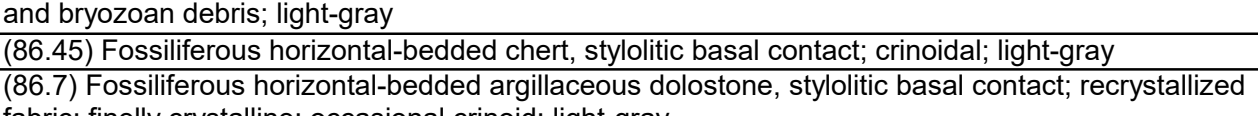

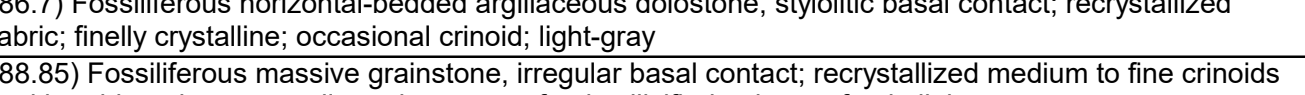

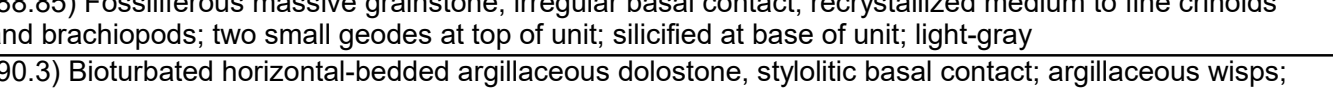

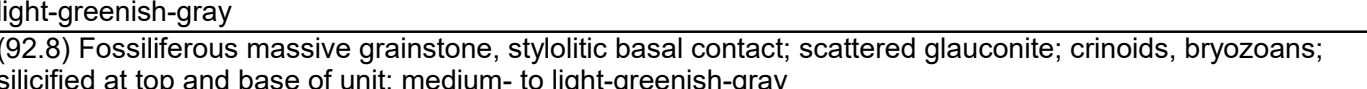

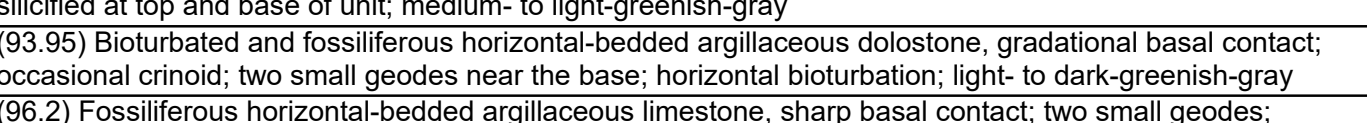

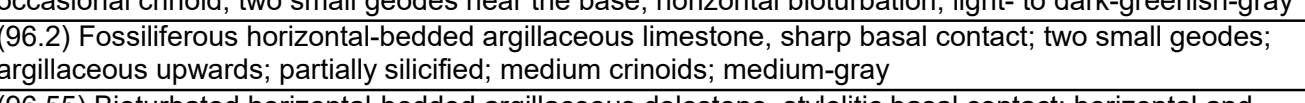

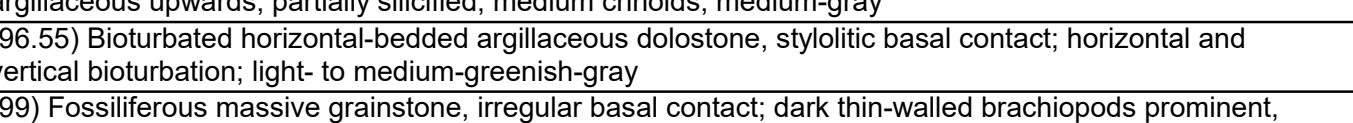

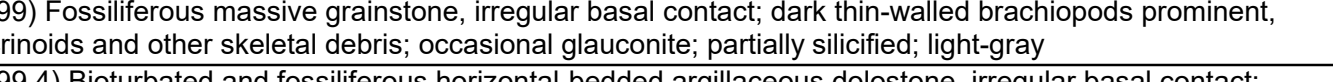

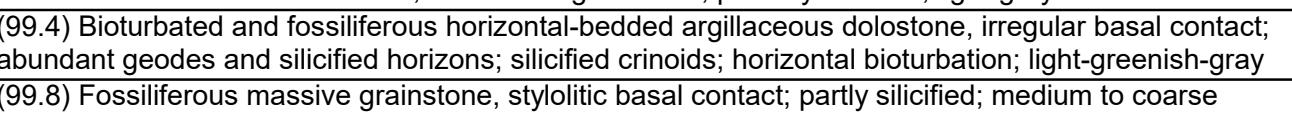

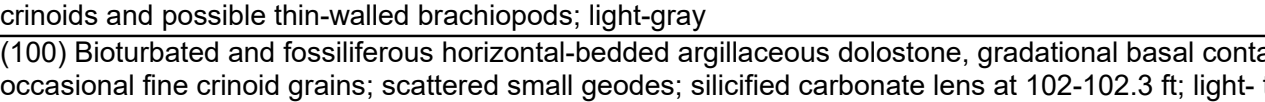
(104.45)

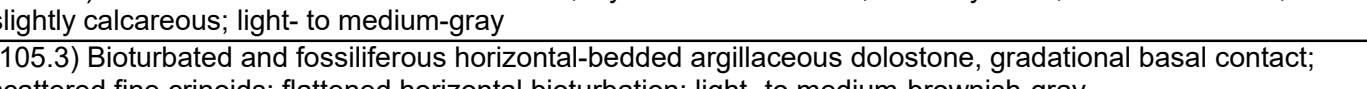

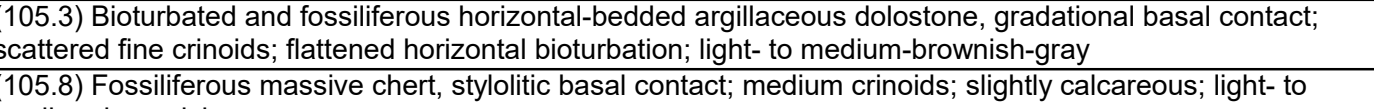

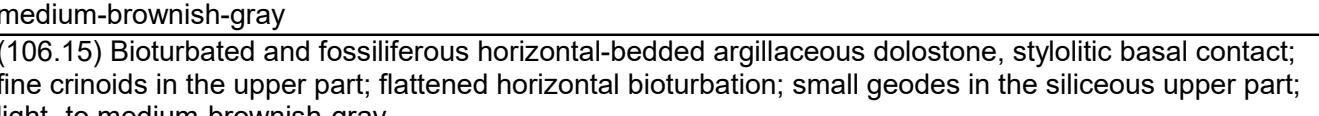

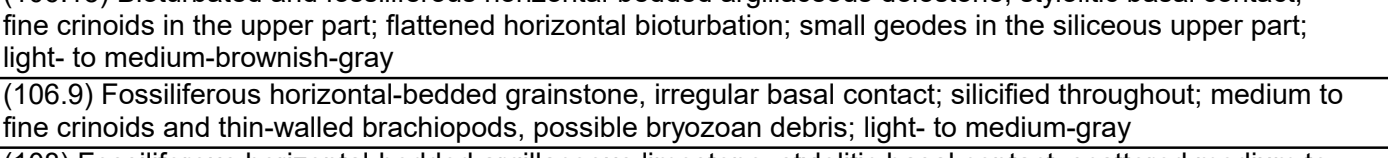

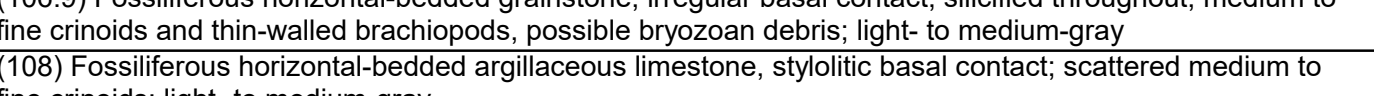

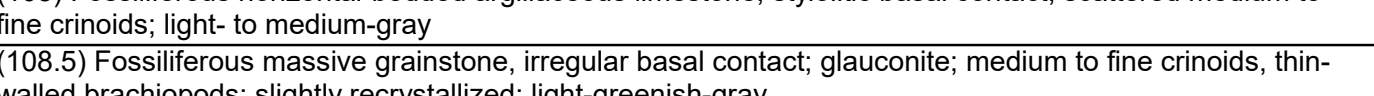

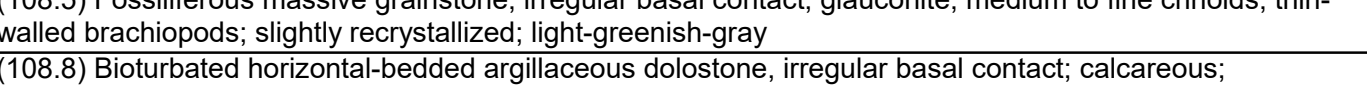

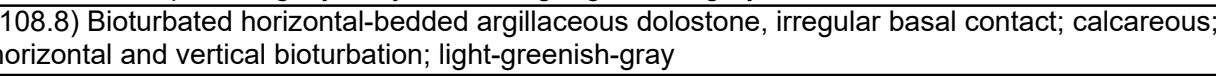

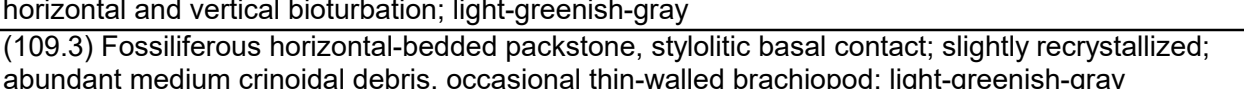

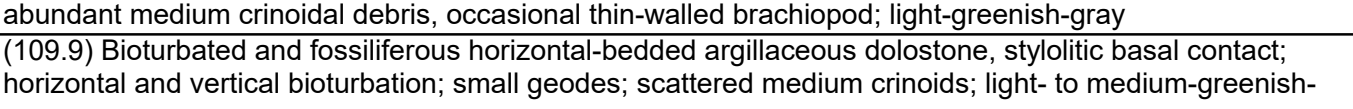

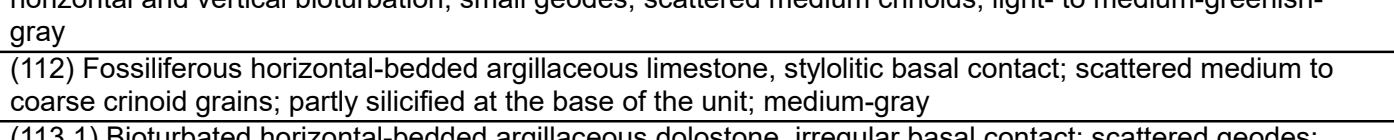

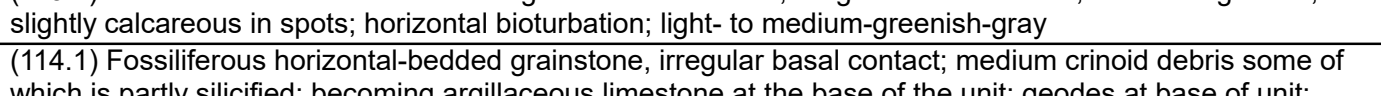

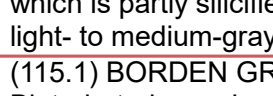
15.1) BORDENGROUP, Edwardvillil Formation
Bioturbated massive sandstone: horizontal and vertical bioturbation; some thinly laminated zones; lower to Bioturbated massive sandstone; horizont
upper very fine grained; medium-gray 


\begin{tabular}{|c|c|c|}
\hline \begin{tabular}{|l} 
I N Number: \\
Site Name: \\
Location: \\
Elevation:
\end{tabular} & 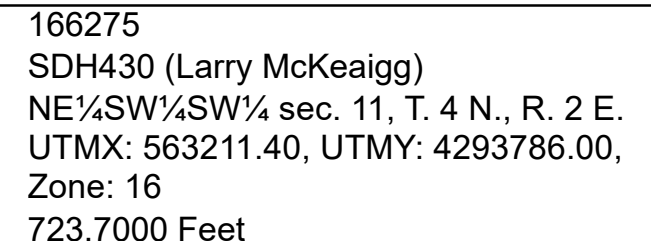 & 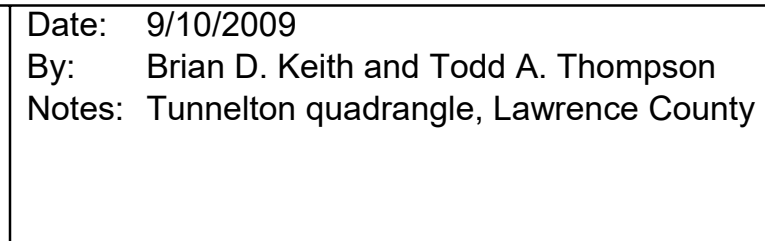 \\
\hline
\end{tabular}
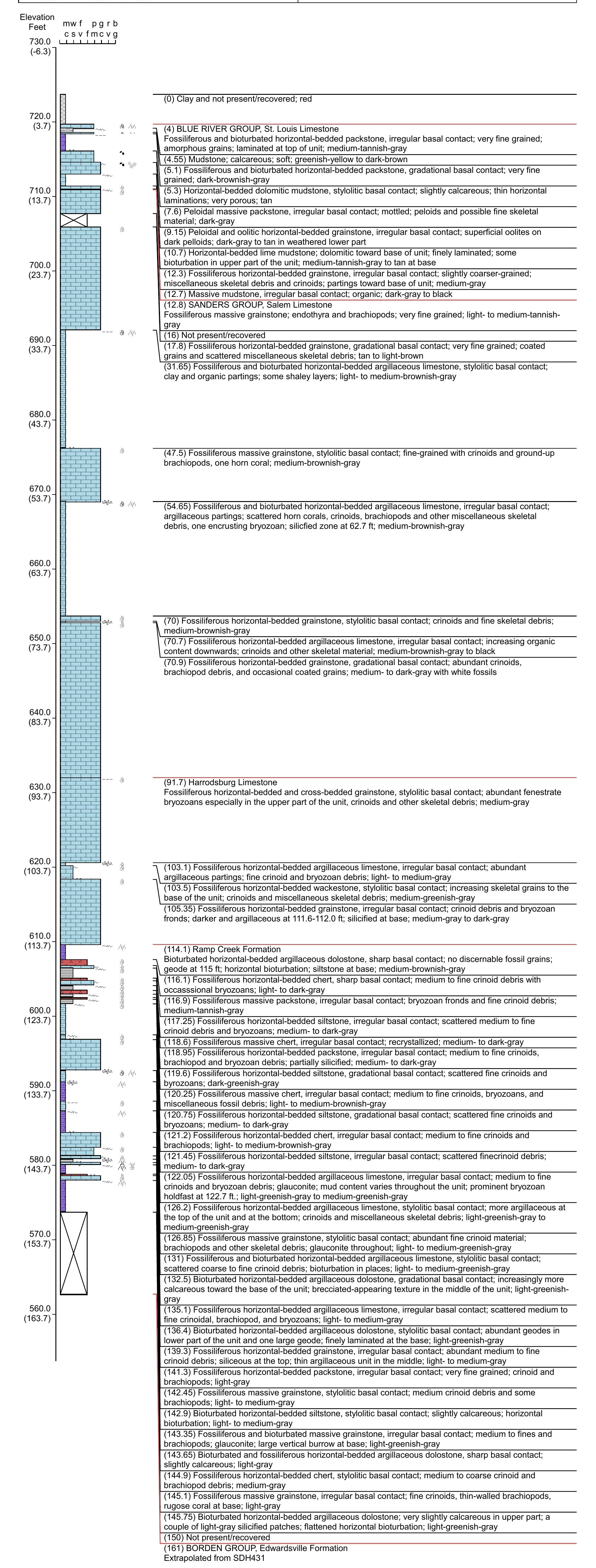


\begin{tabular}{|c|c|c|}
\hline \begin{tabular}{|l} 
ID Number: \\
Sitit Name: \\
Location:
\end{tabular} & 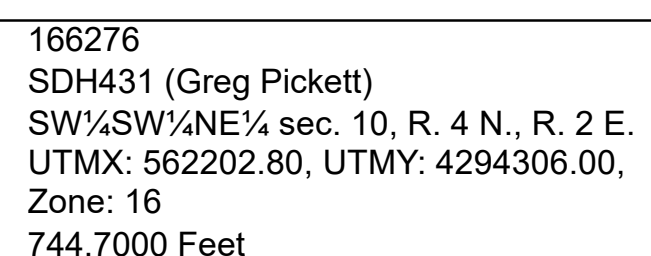 & 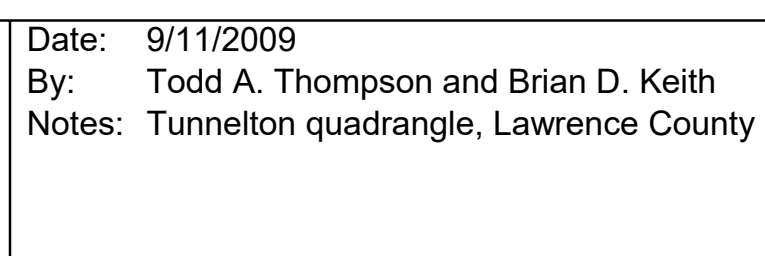 \\
\hline
\end{tabular}
Elevation $\min f \mathrm{pgrb}$
Feet

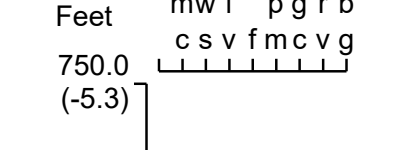

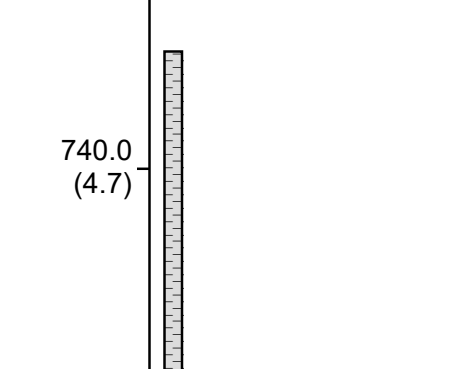
(0) Clay and not presentrecovered; $5 \mathrm{ftbrown} \mathrm{silty} \mathrm{clay,} 9.7 \mathrm{ft}$. reddish brown clay
730.0
220.0
Fossiliferous massive grainstone, styllititi basal contact; crinoids, blastoids, brachiopods; weathered
lannish-gray at top to medium-tannish-gray at the base
Fossiliferous horizontamelebtoned grainstone,
fenestrate bryozzans, scattered crinoids
(2305) Fosill

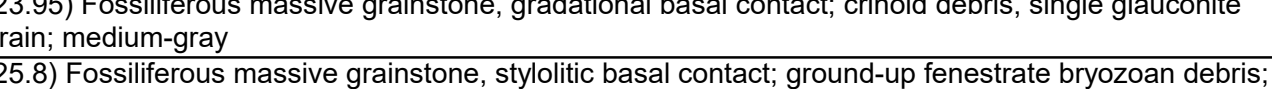
$\frac{\text { 2ery fine grained }}{(27.65) \text { Fossiliferous massive grainstone, irregular basal contact; ground-up crinoid debris; medium-gray }}$
(31.9) Fossiliferous horizontal-bedded argillaceous limestone, gradational basal contact; abundant
\begin{tabular}{l}
710.0 \\
$(34.7)$ \\
\hline
\end{tabular}

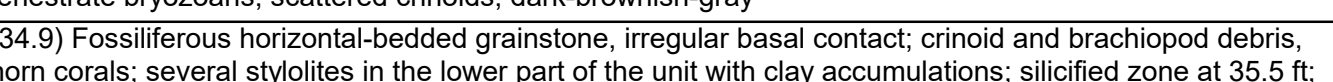
medium-gray

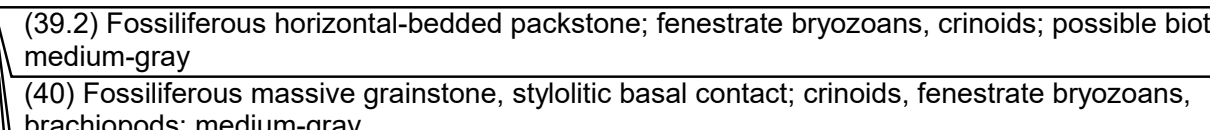
brachiopods; medium-gray
(40.9) Fossiliferous horizontal-bedded packstone; bryozoans, thin-walled brachiopods, bryozoan holdffast in

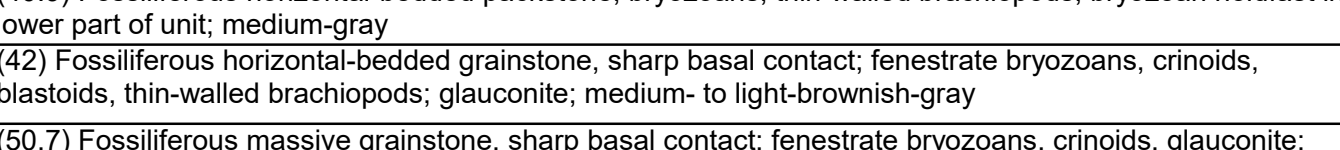
\begin{tabular}{l}
700.0 \\
$(44.7)$ \\
\hline
\end{tabular}
690.0
54.7
5.7

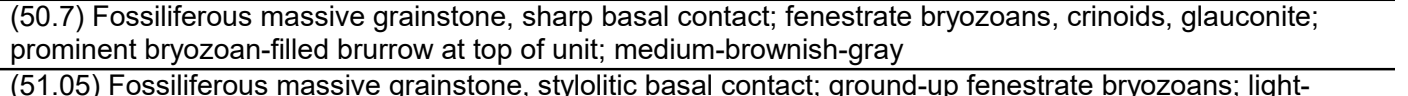

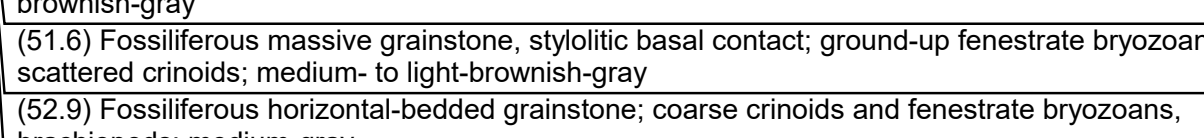

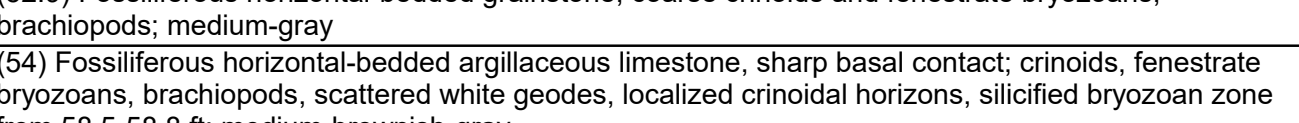

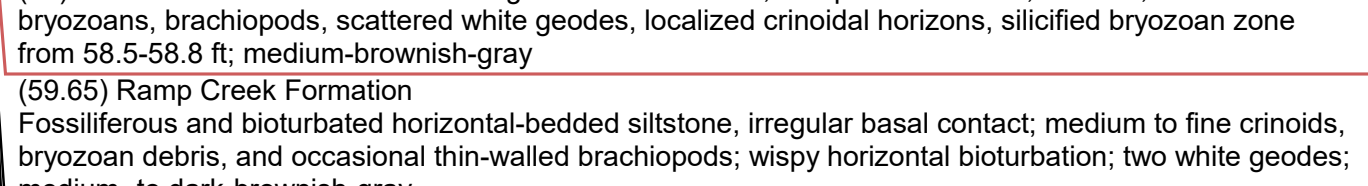
680.0

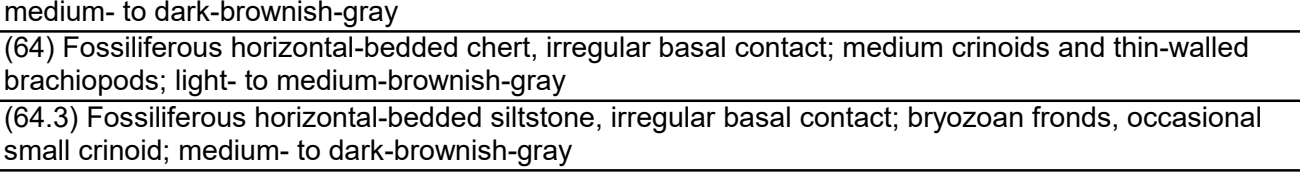

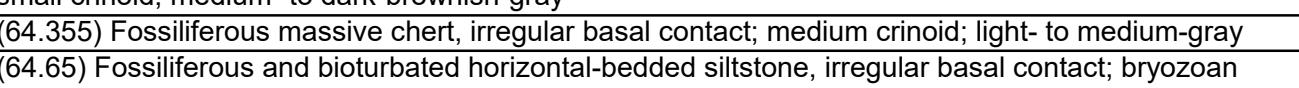

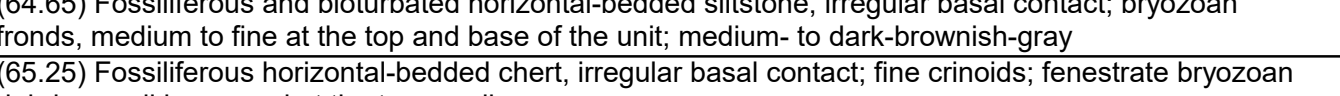
$\begin{array}{r}660.0 \\ (84.7) \\ \hline\end{array}$

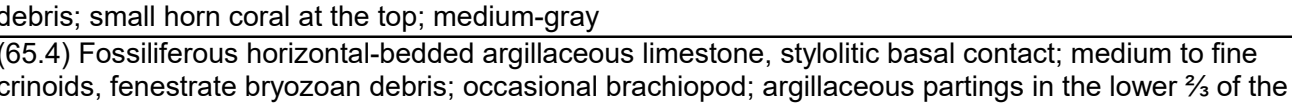

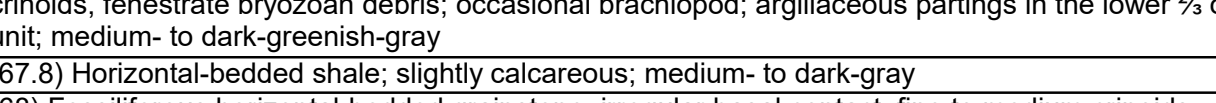

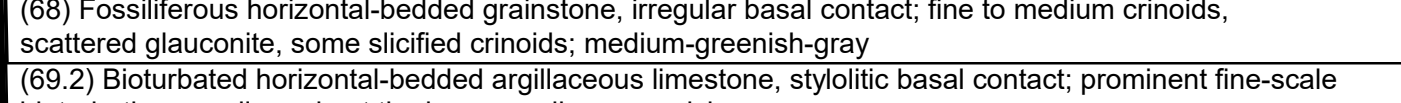

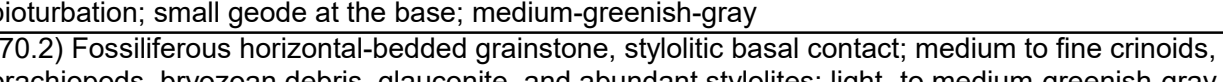

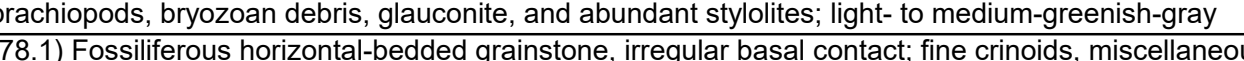

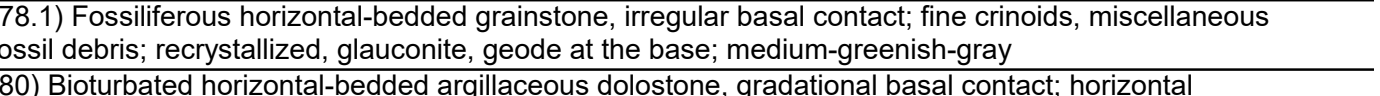

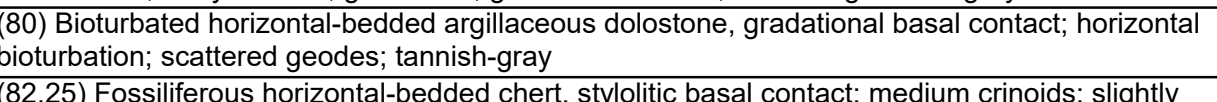

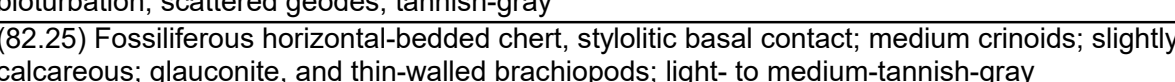
83) Fossilifierous horizontal-bedded argillaceous limestone, stylliticic basal contact; scattered fine to

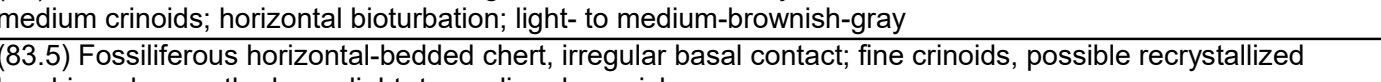

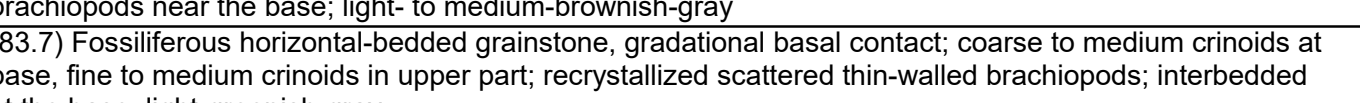

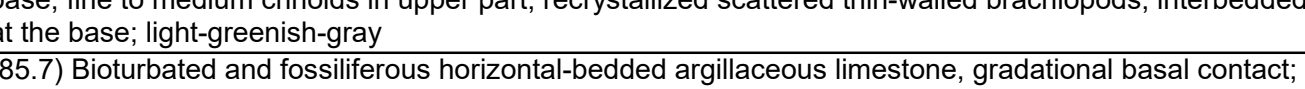

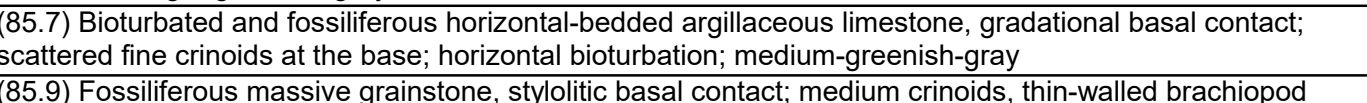

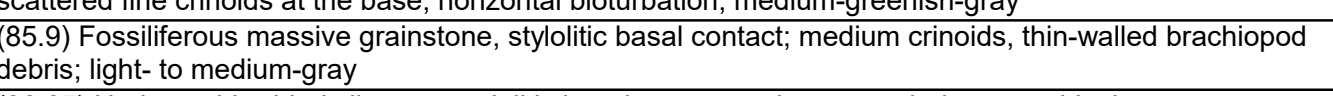

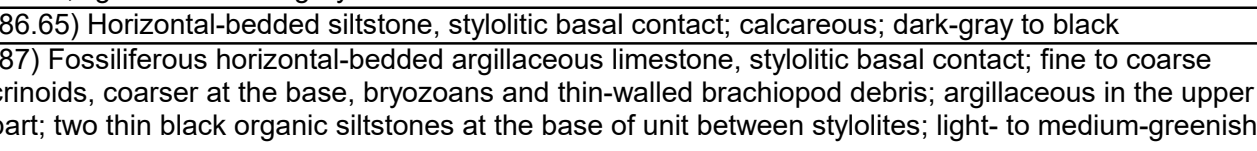

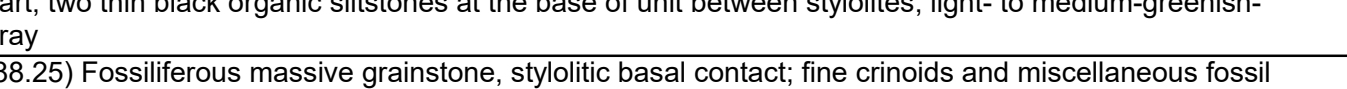

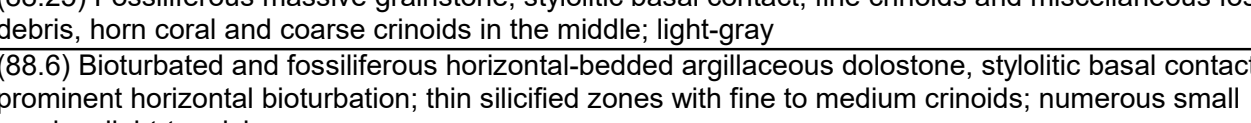
prominent horizontal bioturb
geodes; light-tannish-gray
(107) BORDEN GROP, E. E

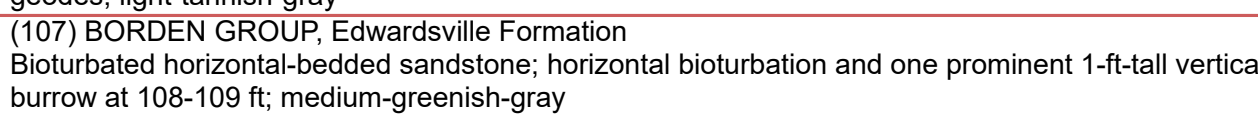


\begin{tabular}{|l|l|}
\hline ID Number: 166277 & Date: \\
Site & 9/14/2009
\end{tabular}

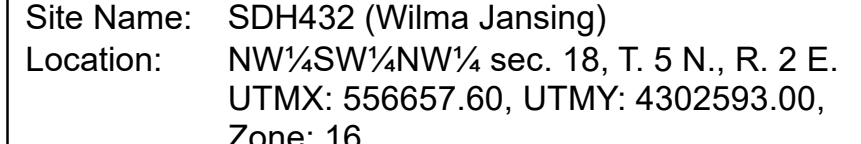

By: Brian D. Keith and Todd A. Thompson Zone: 16

Elevation: $\quad 777.8000$ Feet

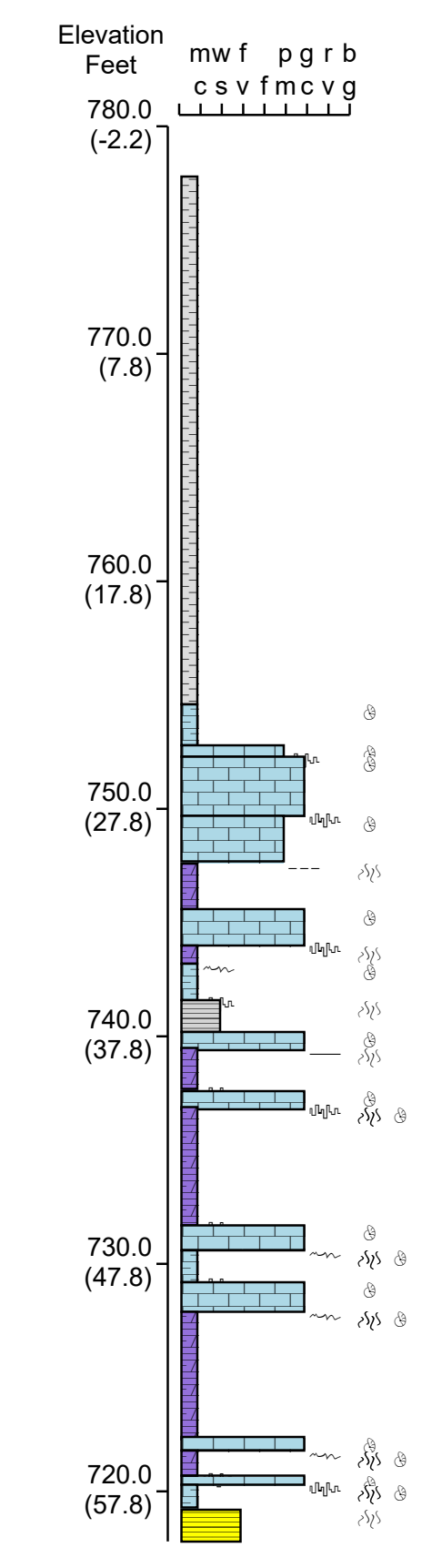

(0) Clay and not presentrecovered; Brown silty clay over red clay, then brown clay with black streaks

(23.2) SANDERS GROUP, Ramp Creek Formation

Fossiliferous horizontal-bedded argillaceous limestone, irregular basal contact; scattered crinoids, (25) Fopod debris, geode at bass layers; glauconite, light-greenish-gray
(25.5) Fossiliferous massive and cross-bedded grainstone, with a stylolitic basal contact; crinoids,

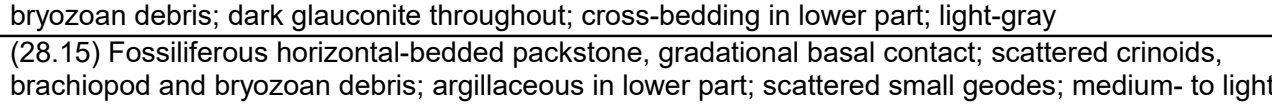
brachiopod and bryozoan debris; argillaceous in lower part; scattered small geodes; medium-to light-
gra. contact, top few inches are calcareous and gradalanhal
horizzontal bedding in upper part of unitit light-greenish-gray

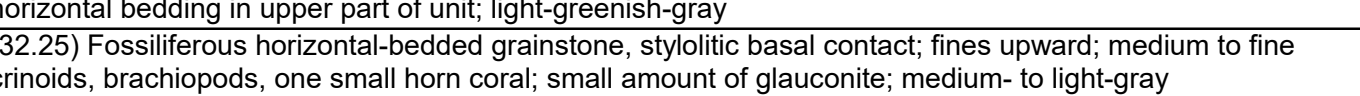

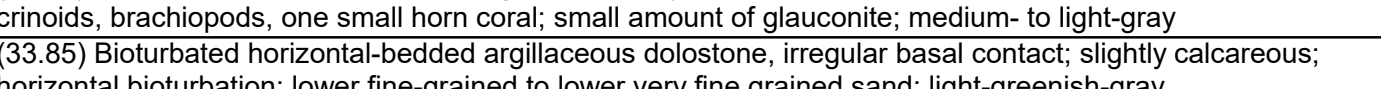

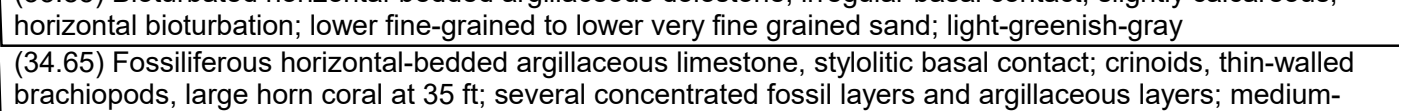

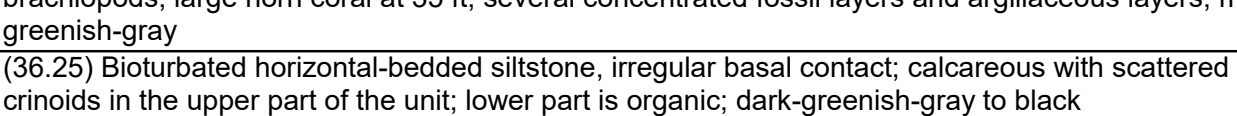

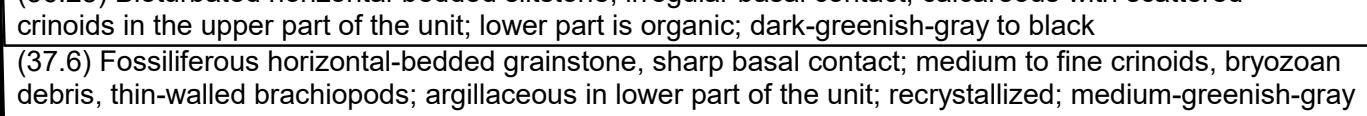

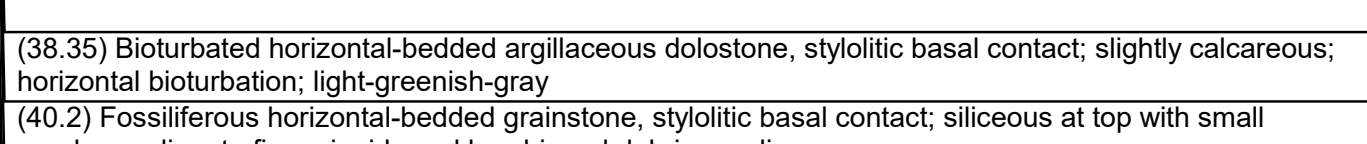

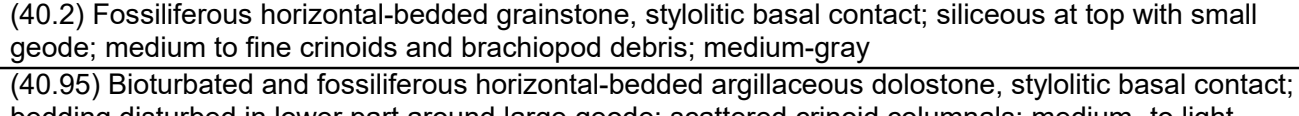

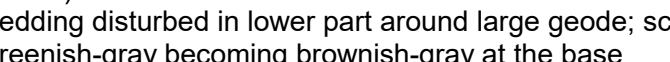

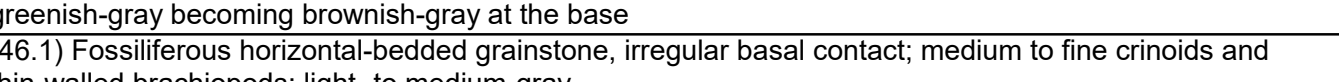

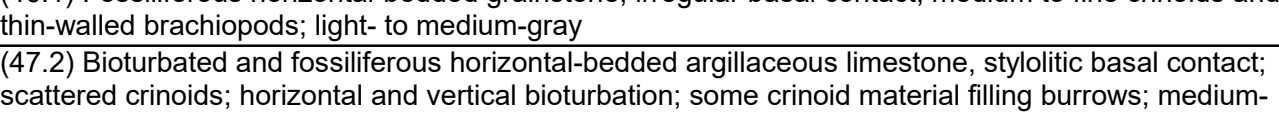
scattered crinoids; horizontal and vertical bioturbation; some crinoid material flliling burrows; medium-
brownish-gray
(48.6) Fossiliferous horizontal-bedded grainstone, iregular basal contact argillaceous layer in middle of the unit; large thin-walled brachiopods and medium to fine crinoid debris; medium-to dark-gray
(49.9) Bioturbated and fossiliferous horizontal-bedded argillaceous dolostone, gradational basal contact-

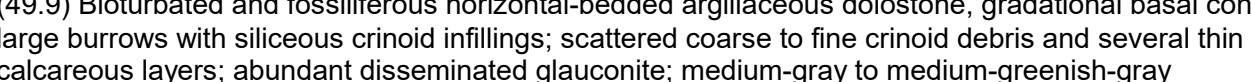
calcareous layers; abundant disseminated glauconite; medium-gray to medium-greenish-gray
(55.45) Fossiliferous massive grainstone, irregular basal contact; may be argillaceous in upper part of the

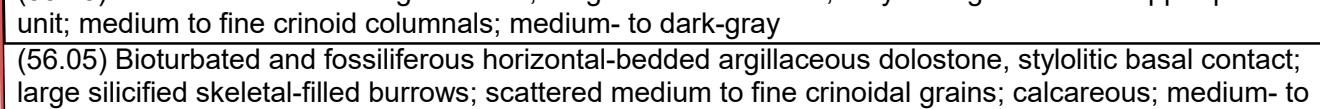
dark-gray
(57.15) Fossiliferous cross-bedded grainstone, styllitic basal-contact; coarse to medium crinoid debris;

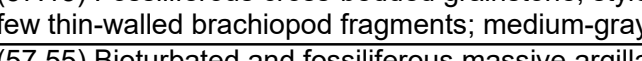
(57.55) Bioturbated and fossiliterous massive argillaceous limestone, irregular basal contact; calcareous;
smanl geode, fine fossil debris at base; horizontal and vertical burrows; medium- to dark-gray

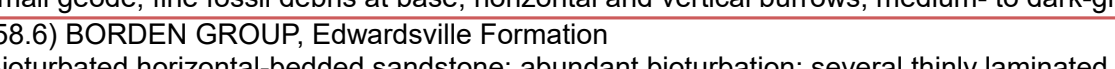
ivoturbated horizontal-bedded sandstones, abundant biturbation; several thinly laminated zones; vertical
and horizontal burrows; upeer very fine grained to lower very fine grained sand; medium-gray 


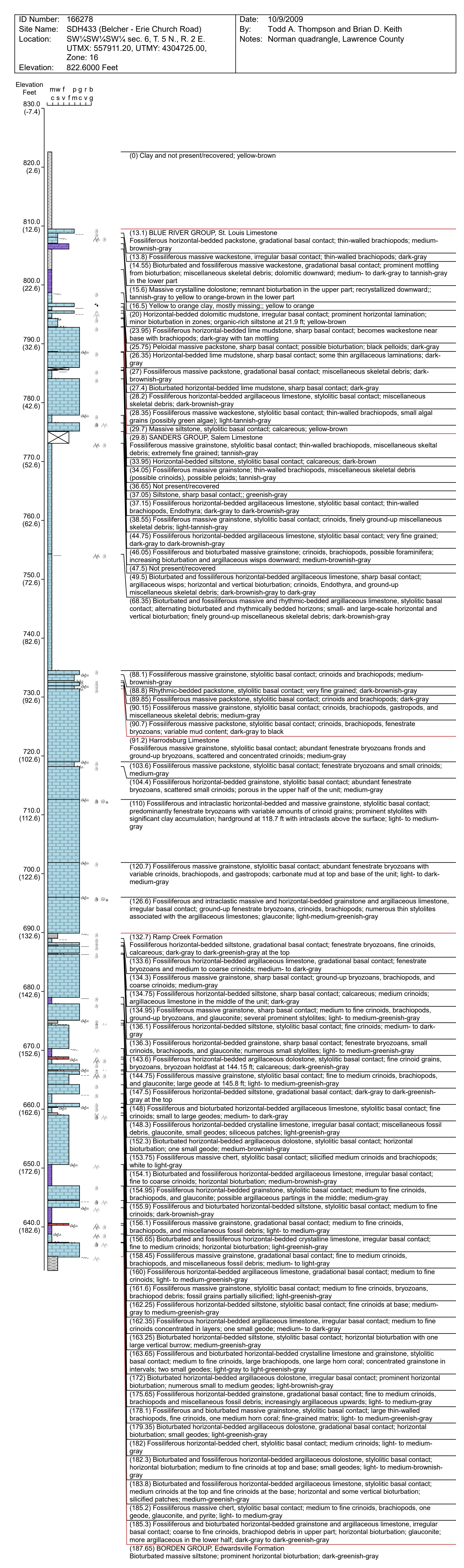




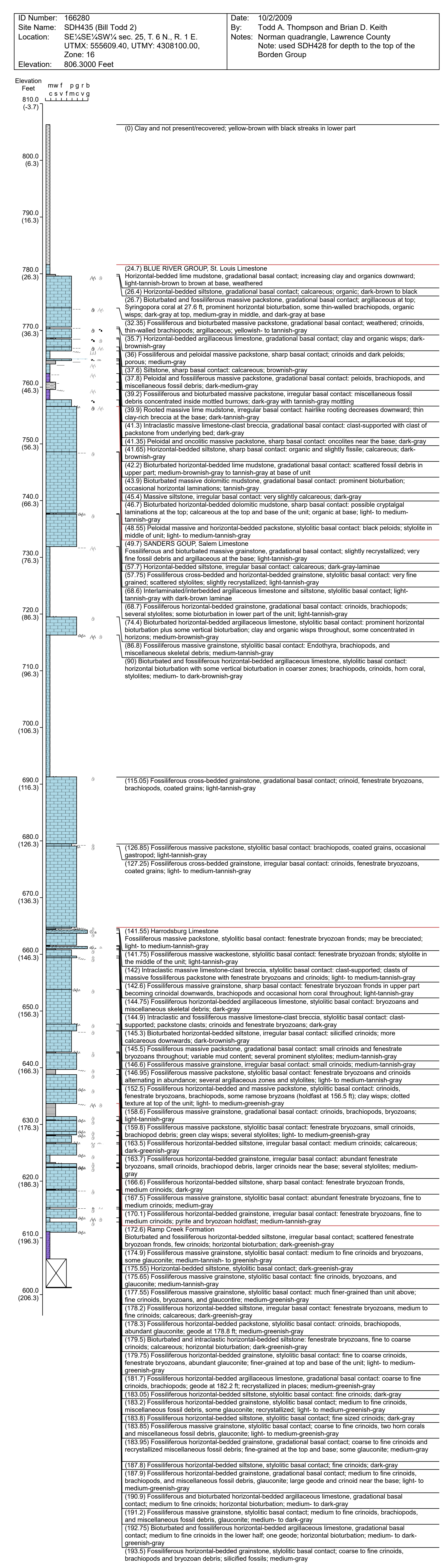


\begin{tabular}{ll}
\hline ID Number: & 166283 \\
Site Name: & SDH438 (Daltor
\end{tabular}

$\begin{array}{ll}\text { Date: } & 10 / 27 / 2009 \\ \text { By: } & \text { Brian D. Keith }\end{array}$

UTMX: 55175580 , UT. 4 N., R. 1 E.

\section{By: Brian D. Keith
Notes: Bedford East}

Hole abandoned due to to bawrence County

\begin{tabular}{ll} 
& Zone: 16 \\
Elevation: & 752.5000 Feet \\
\hline
\end{tabular}

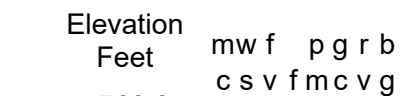

${ }_{(-7.5)}^{760.0}$

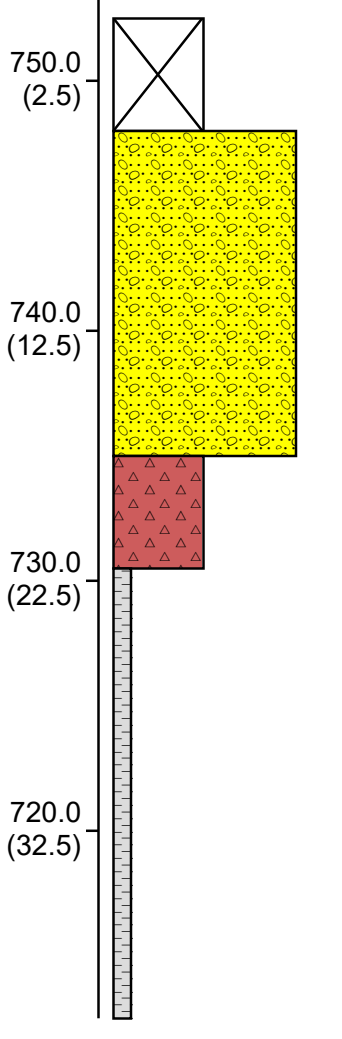

Wresentrecovered; quartz pebbles and red clay, weathered

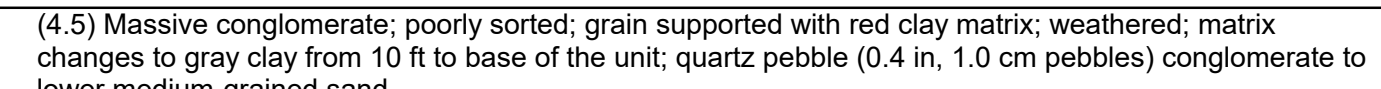

(17.5) Chert; fractured; light-gray

(22) Clay; ; red and yellowish clay with black streaks 


\begin{tabular}{|c|c|c|}
\hline $\begin{array}{l}\text { ID Number: } \\
\text { Site vam: } \\
\text { Location: }\end{array}$ & 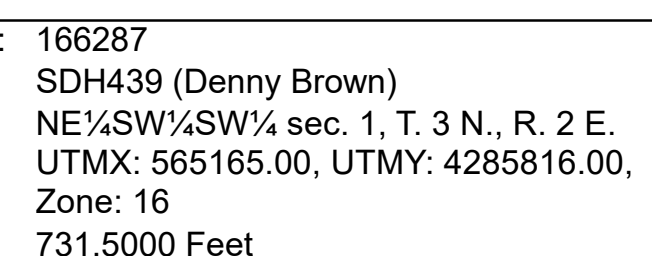 & $\begin{array}{l}\text { Date: } 9110 / 2010 \\
\text { By: } \\
\text { Byran D. Keith and Todd A. Thompson } \\
\text { Notes: Smediley quadrangle, Washington County }\end{array}$ \\
\hline
\end{tabular}

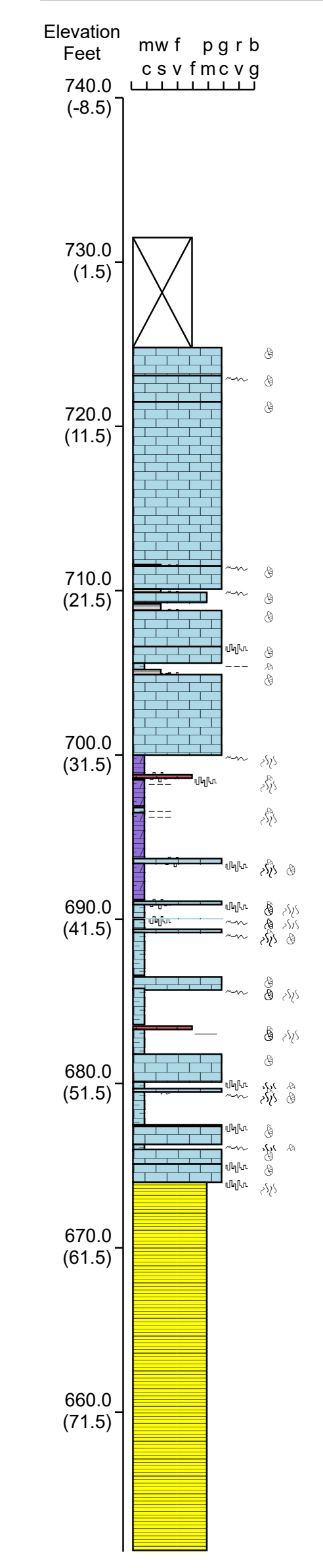

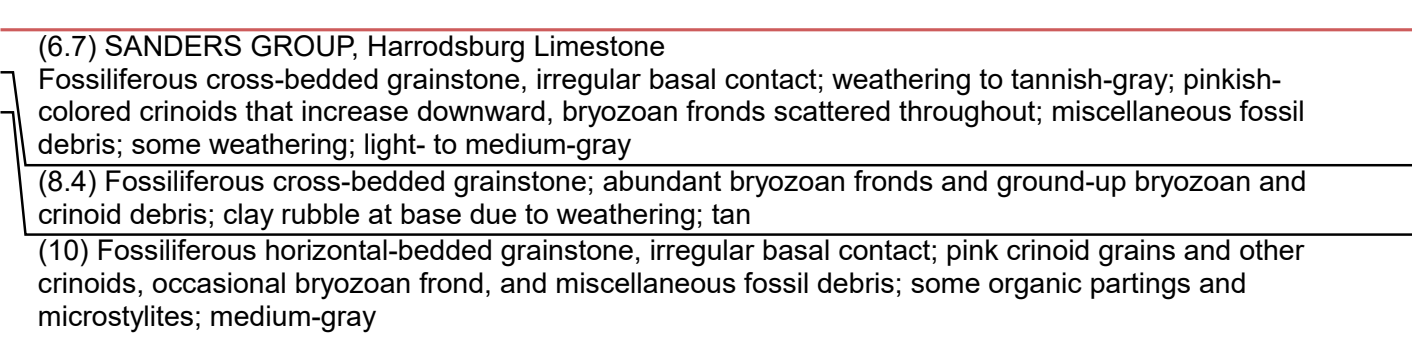

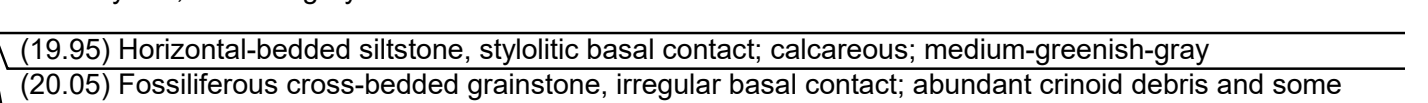

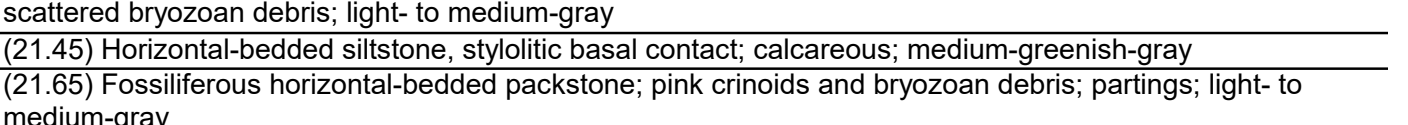

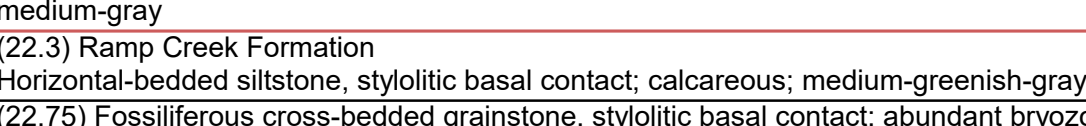

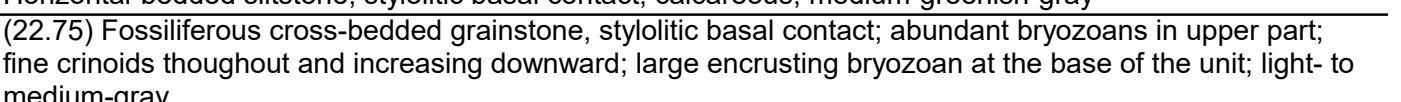

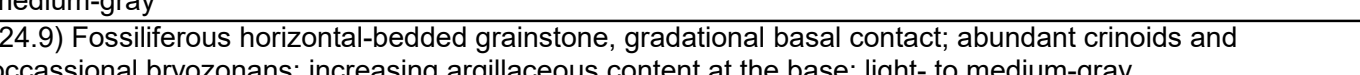

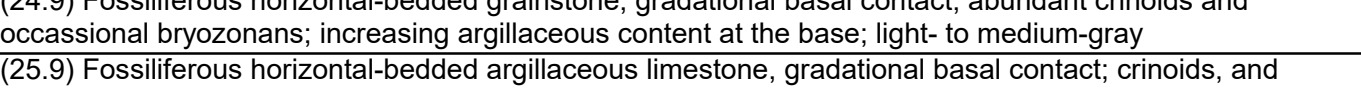

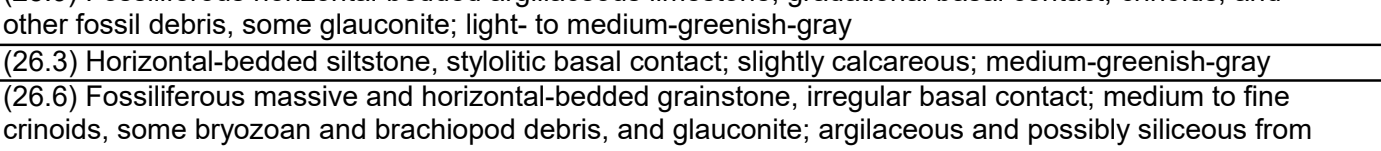

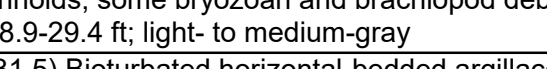

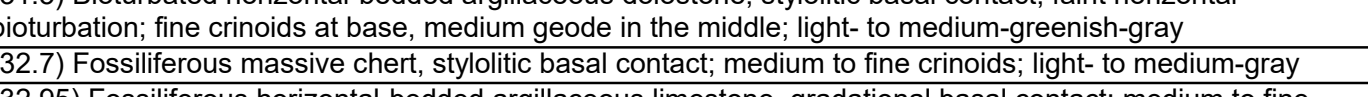

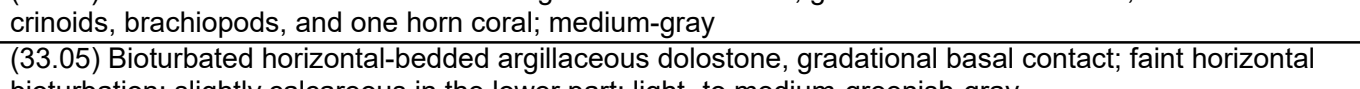

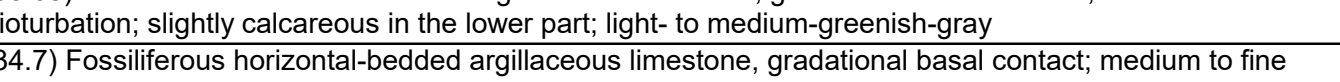

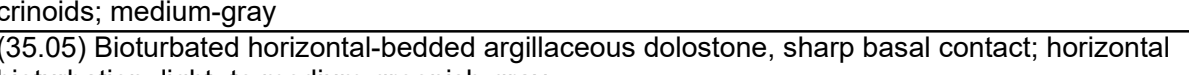

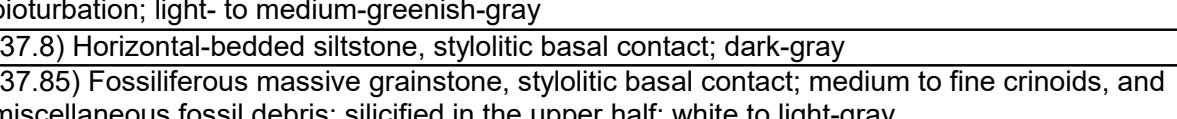
redium to fine crinods in zoness beconing siliceous at the base: ight- to medium-rgenensh-rarat

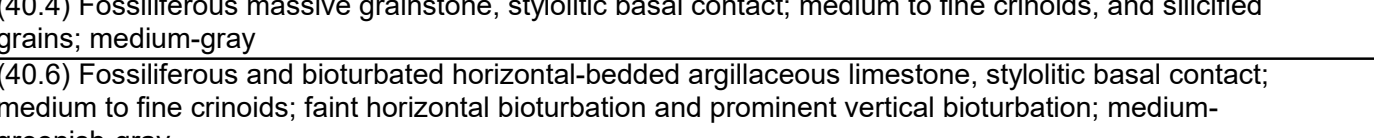

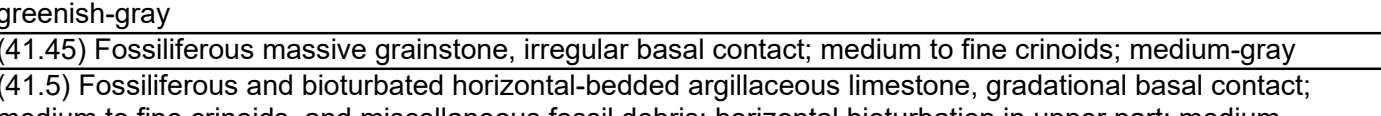

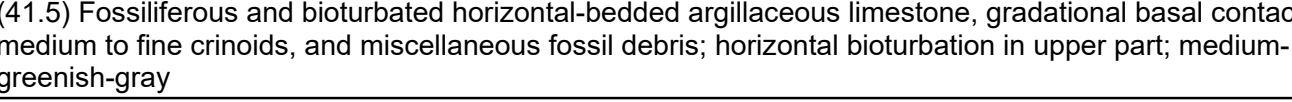

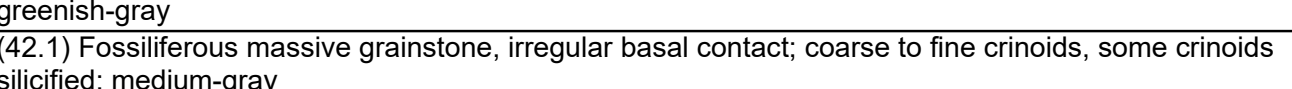

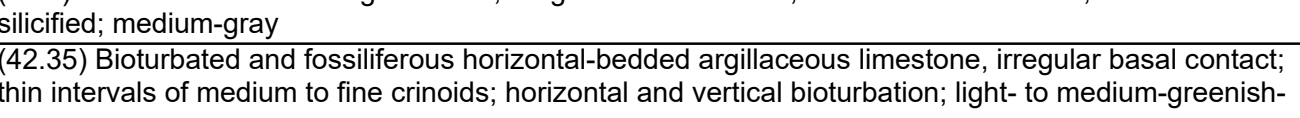

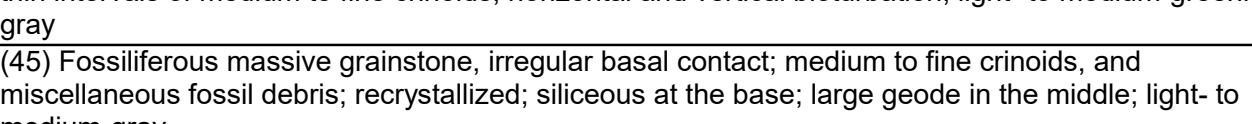

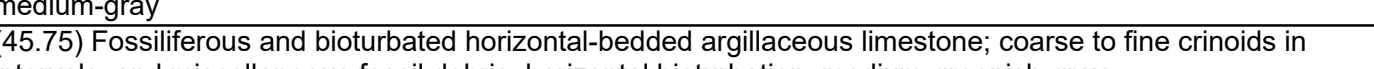

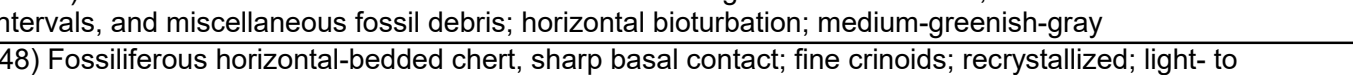

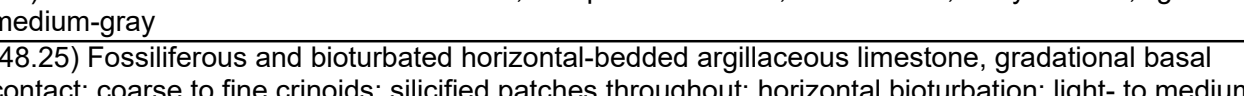
greenish-gray $51.45)$ Bioturbatated and fossiliferous horizontal-bedded argillaceous limestone, irregular basal contact;

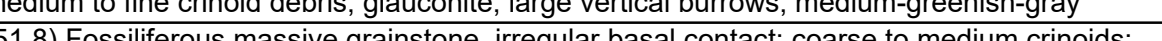

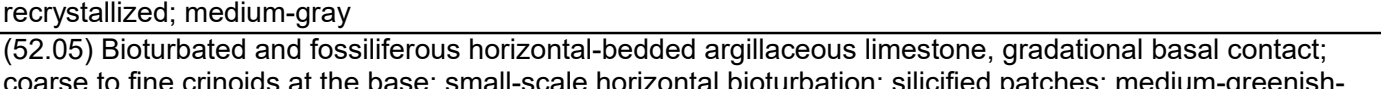
gray
(54) Fossiliferous massive grainstone, styllitic basal contact, medium to fine crinoids; light- to medium-

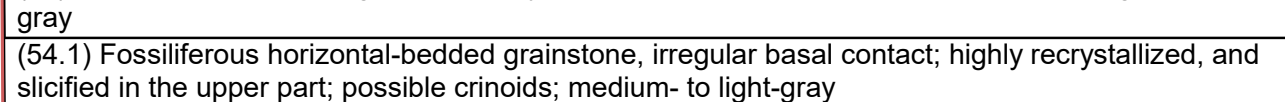
(55.2) Bioturbated and fossiliferous horizontal-bedded argillilaceous limestone, gradational basal contact; (55.5.5) F ossiliferous massive and horizontala-bedded grainstone, stylolitic basal contact; medium to fine
crinids

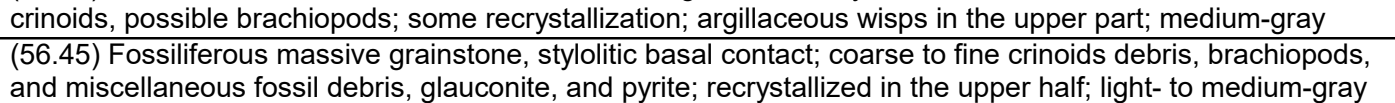

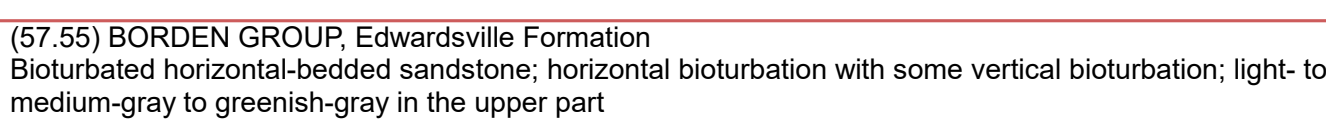




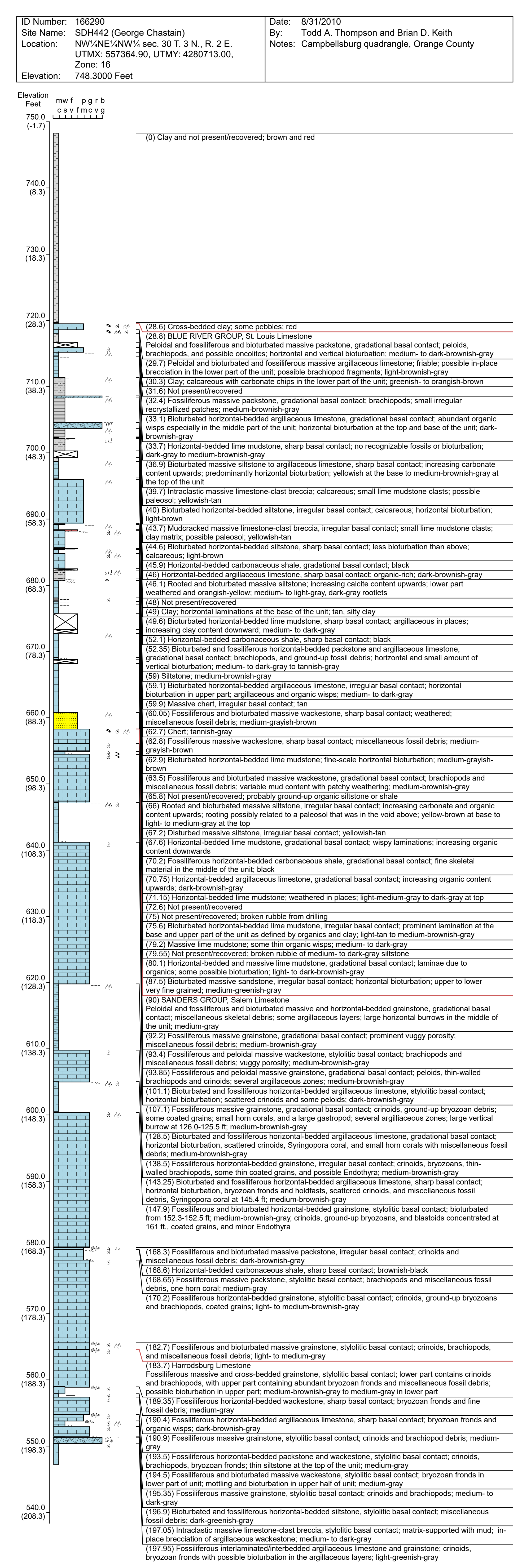




\begin{tabular}{|c|c|c|}
\hline $\begin{array}{l}\text { ID Number: } \\
\text { Site vam: } \\
\text { Location: }\end{array}$ & 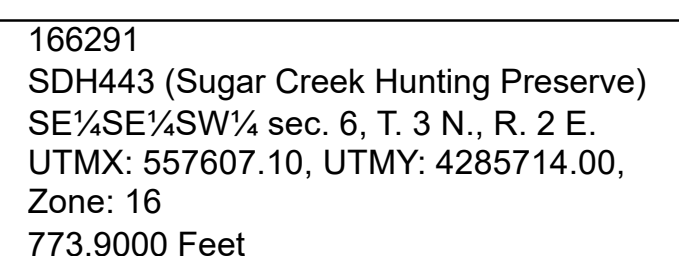 & $\begin{array}{l}\text { Date: } 91 / 212010 \\
\text { By: } \\
\text { Brian D. Keith and Todd A. Thompson } \\
\text { Notes: Campellsburg quadrangle, Lawrence County }\end{array}$ \\
\hline
\end{tabular}

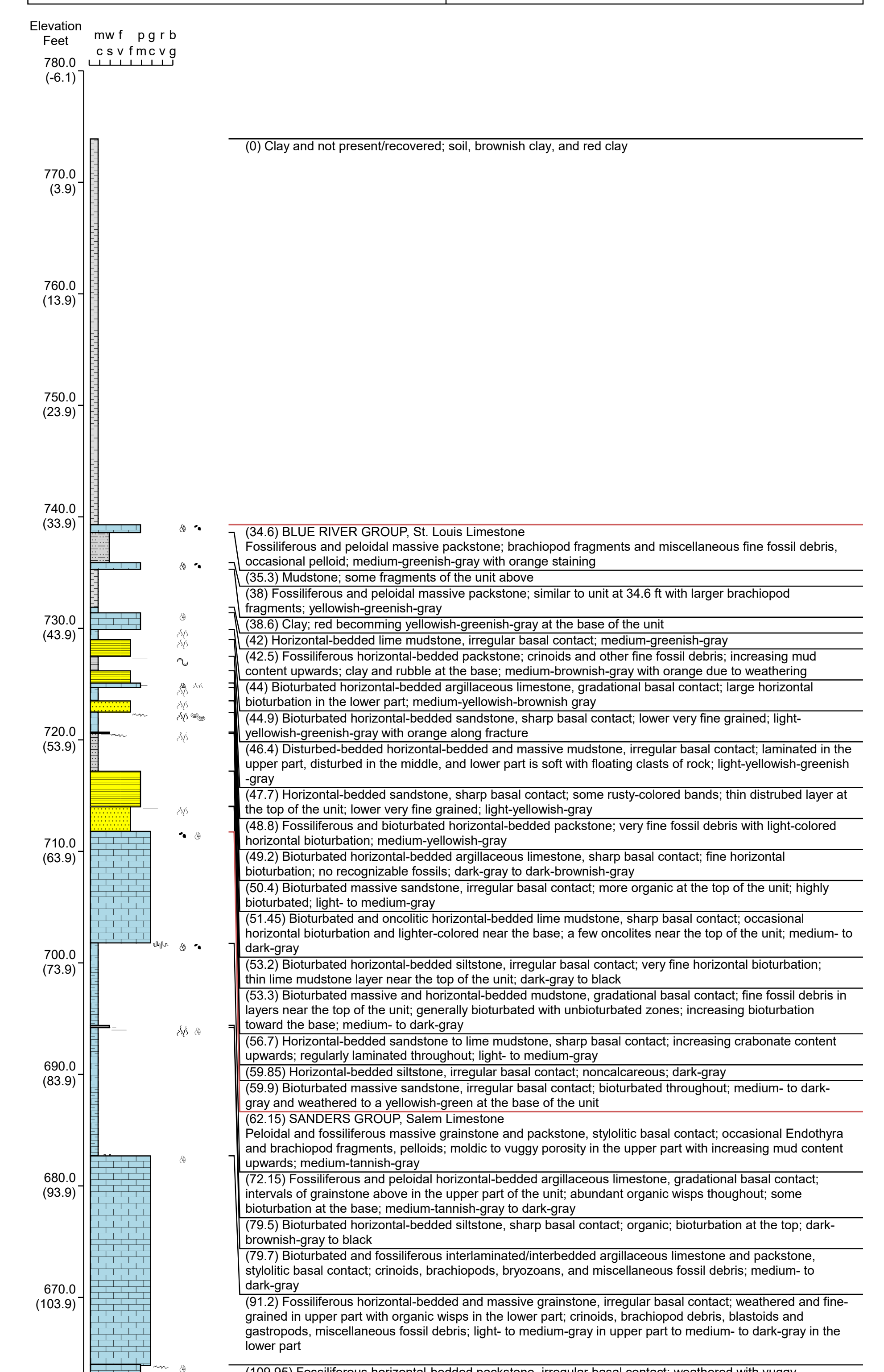

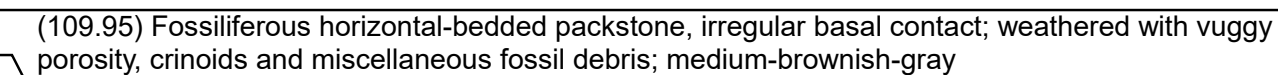

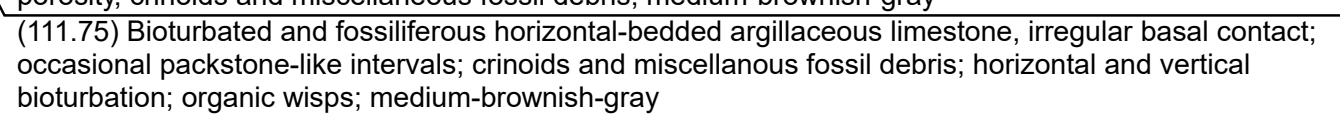

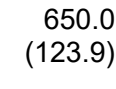

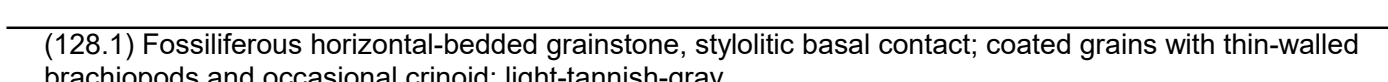
(132.05) Fossiliferous massive g

-gray

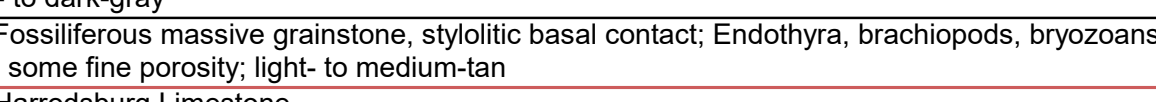

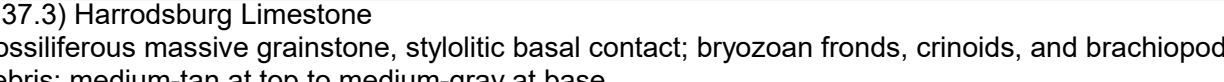
debris; medium-tan at top to medium-gray at base
(1383) Fossiliferous horizontal-bedded wackestone, irregular basal contact; bryozoan fronds; i light-tannish-

gray 138.1$)$ Fossiliferous horizontal-bedded argillaceous limestone, sharp basal contact; very silty and

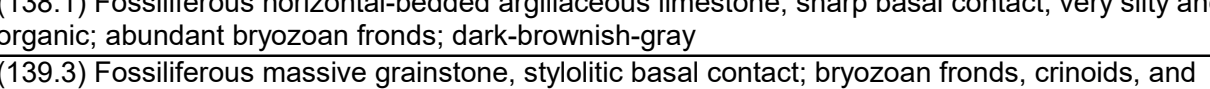

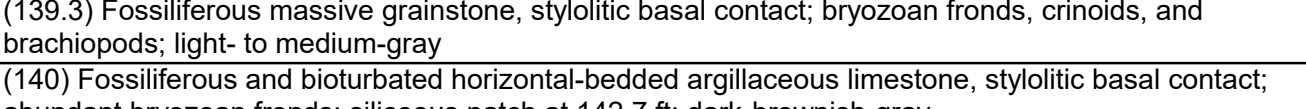
(144) Fossiliferous horizontal-bedded packstone, sharp basal contact; crinoids and miscellaneous fossil

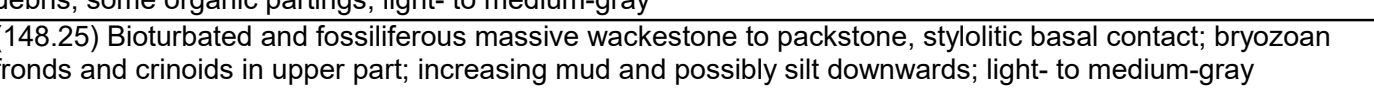

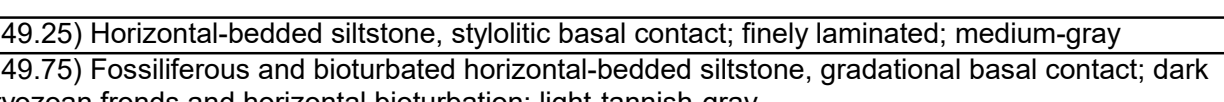

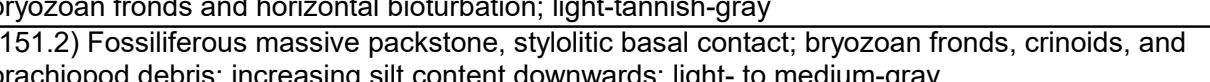

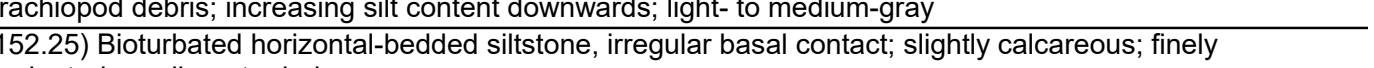

aminated; medium-to dark-gray
152.3) Bioturbated and fossiliferous horizontal-bedded siltstone, gradational basal contact

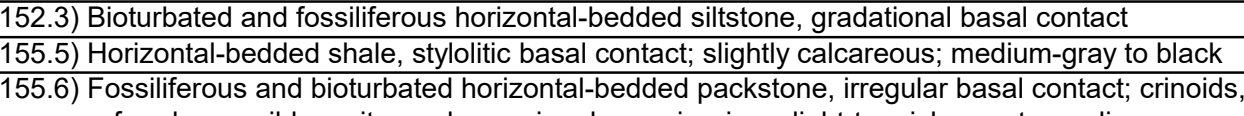

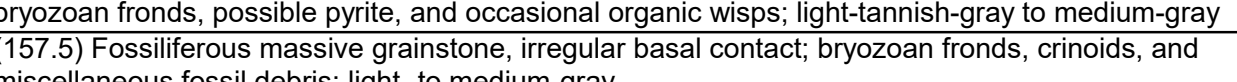

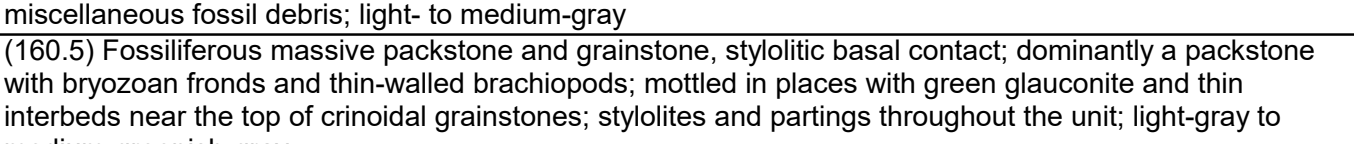

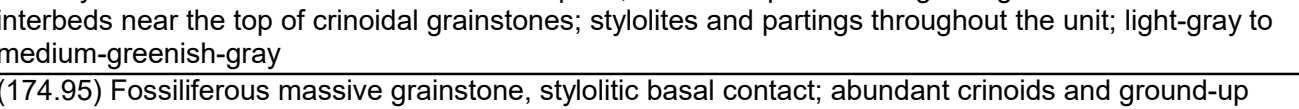

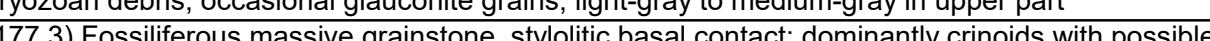

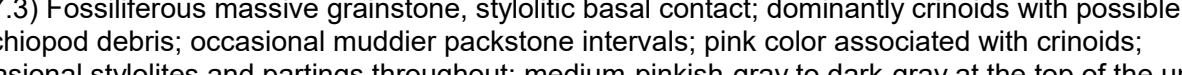
a
(187.

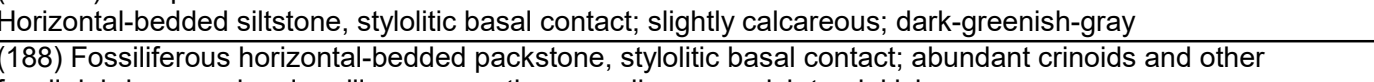

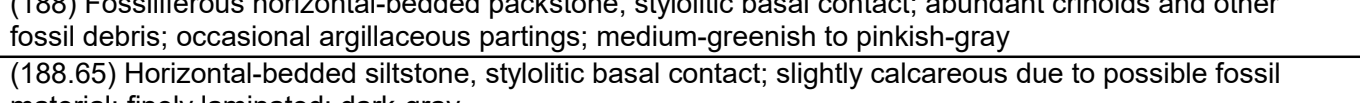

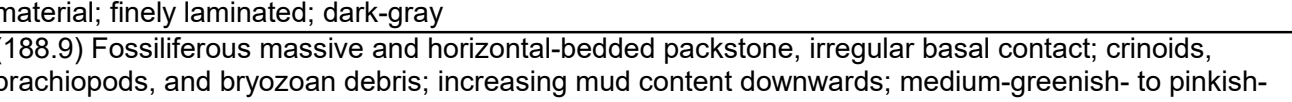

getray
grag

(100.9) Fossiliferous and bitoturbated horizontal-bedded siltstone, gradational basal contact, bitturbated in
calcareous intervals; occasional small crinoids; dark-gray with lighter-gray calcareous intervals in the

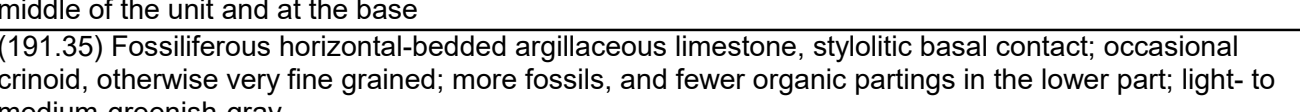
nedium-greenish-gray

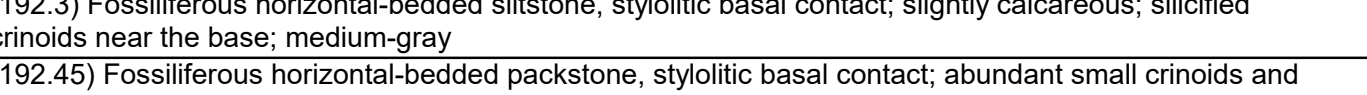

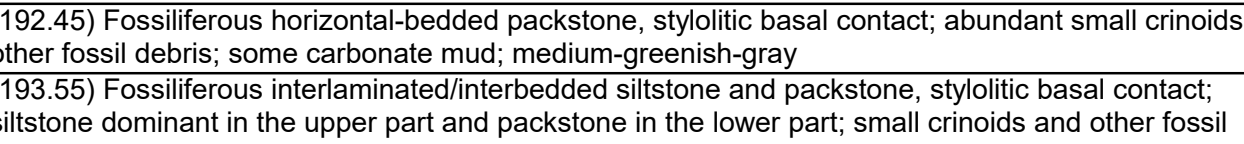

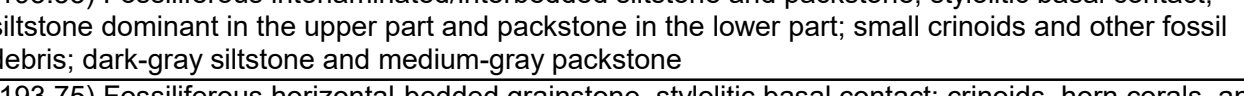

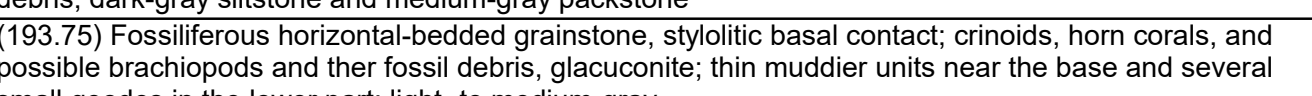

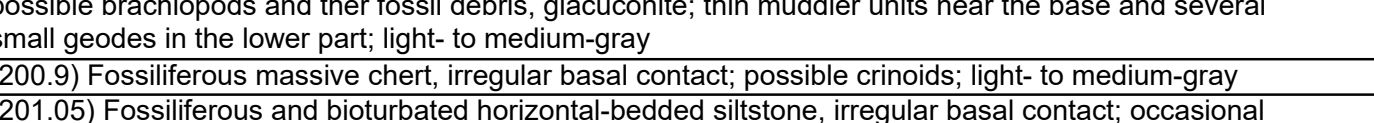

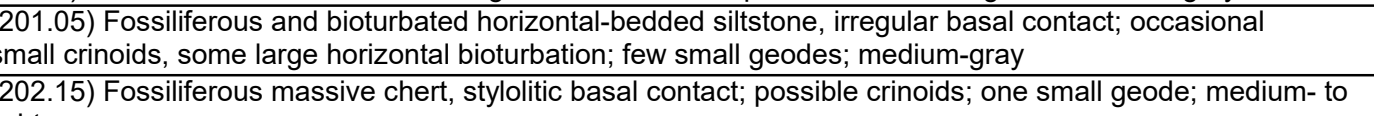
202.15) Fossiliferous massive chert, styllitic basal contact; possible crinoids; one small geode; medium- to
light-gray
202.25) Fossilfiferous massive grainstone, styllitic basal contact; crinoids, and miscellaneous fossil cobris; iliceous: light- to medium-gray
203.05) Fossilififrous horizontal-bedded siltstone, irregular basal-contact; becomes calcareous at the base

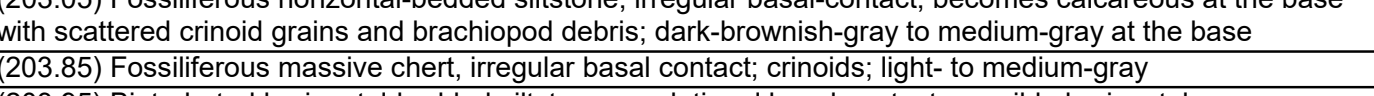

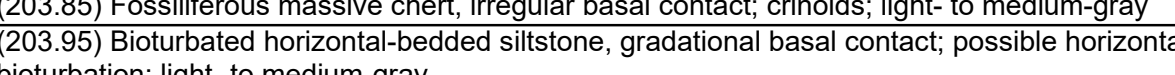

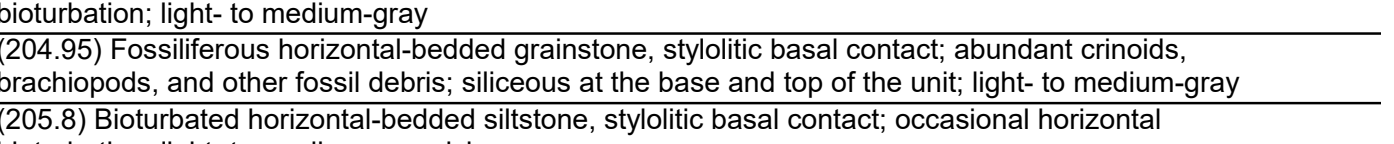

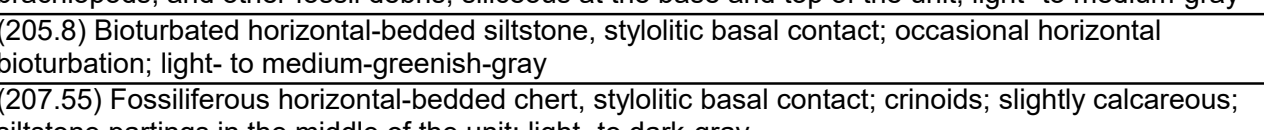

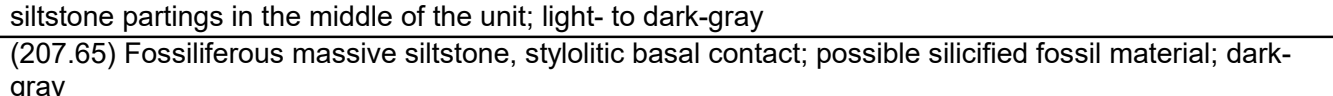




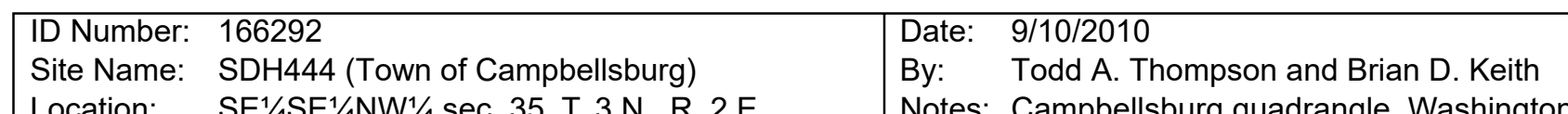

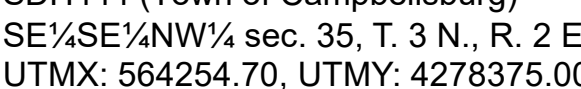 \\ Notes: Campbellsburs quadrangle, Washington County}

Zone: 16
835.2000 Feet

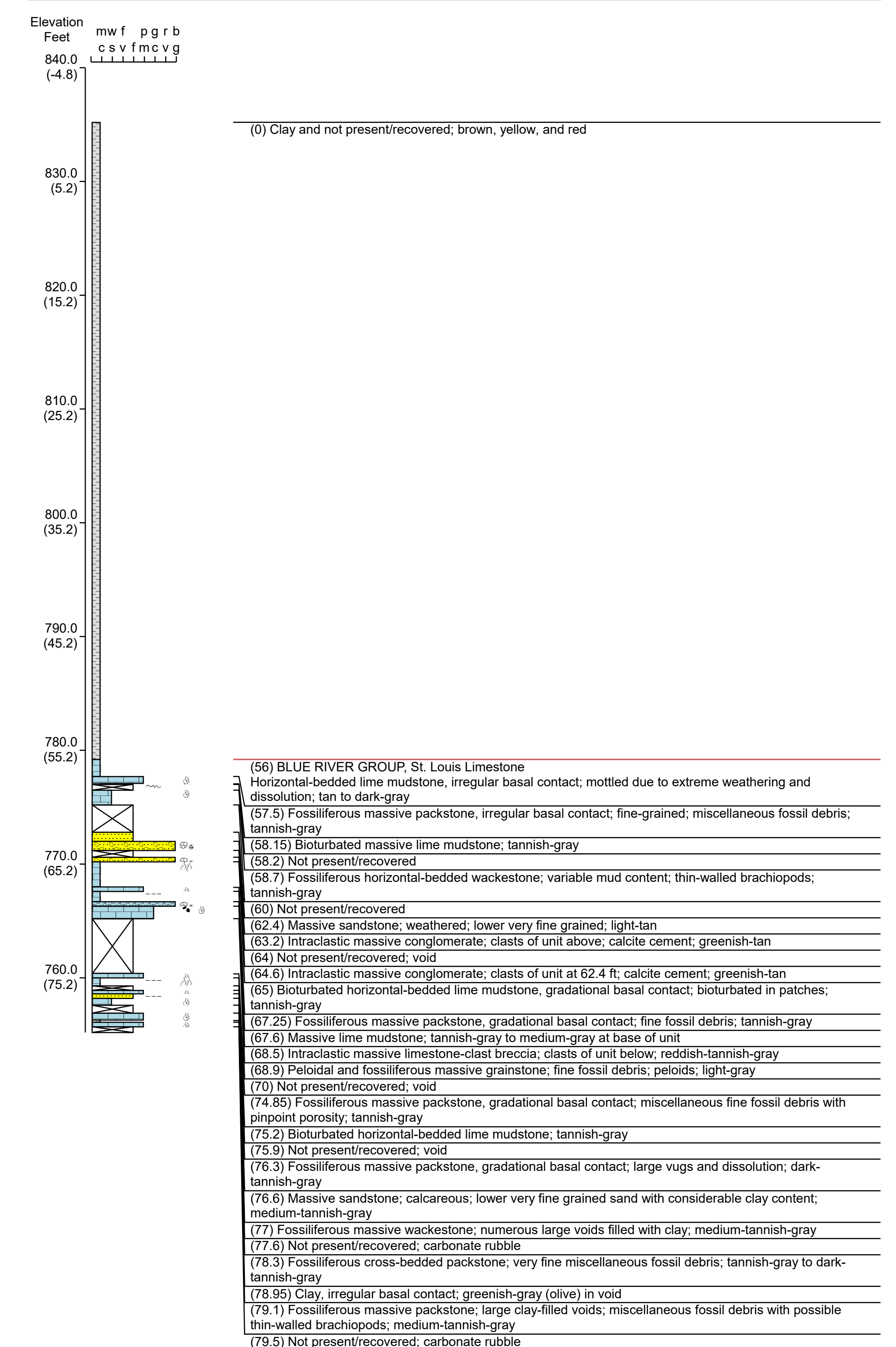




\begin{tabular}{|c|c|c|}
\hline 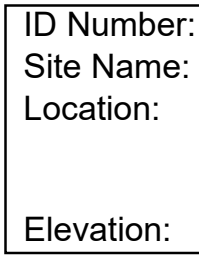 & 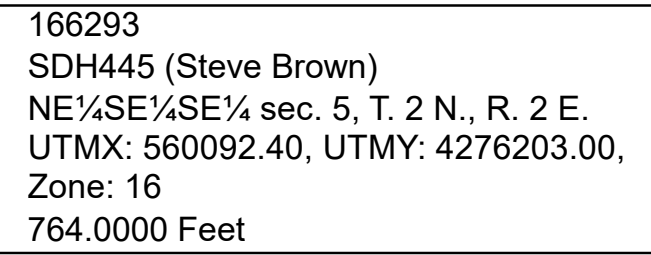 & 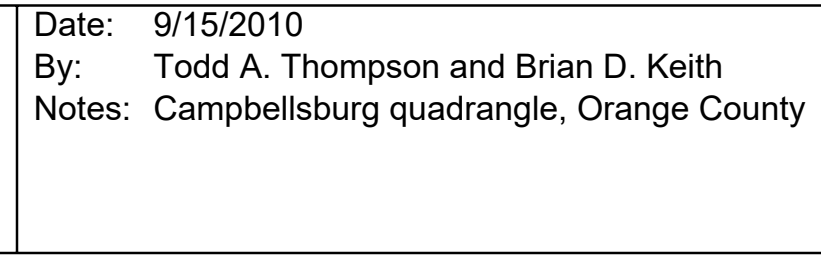 \\
\hline
\end{tabular}

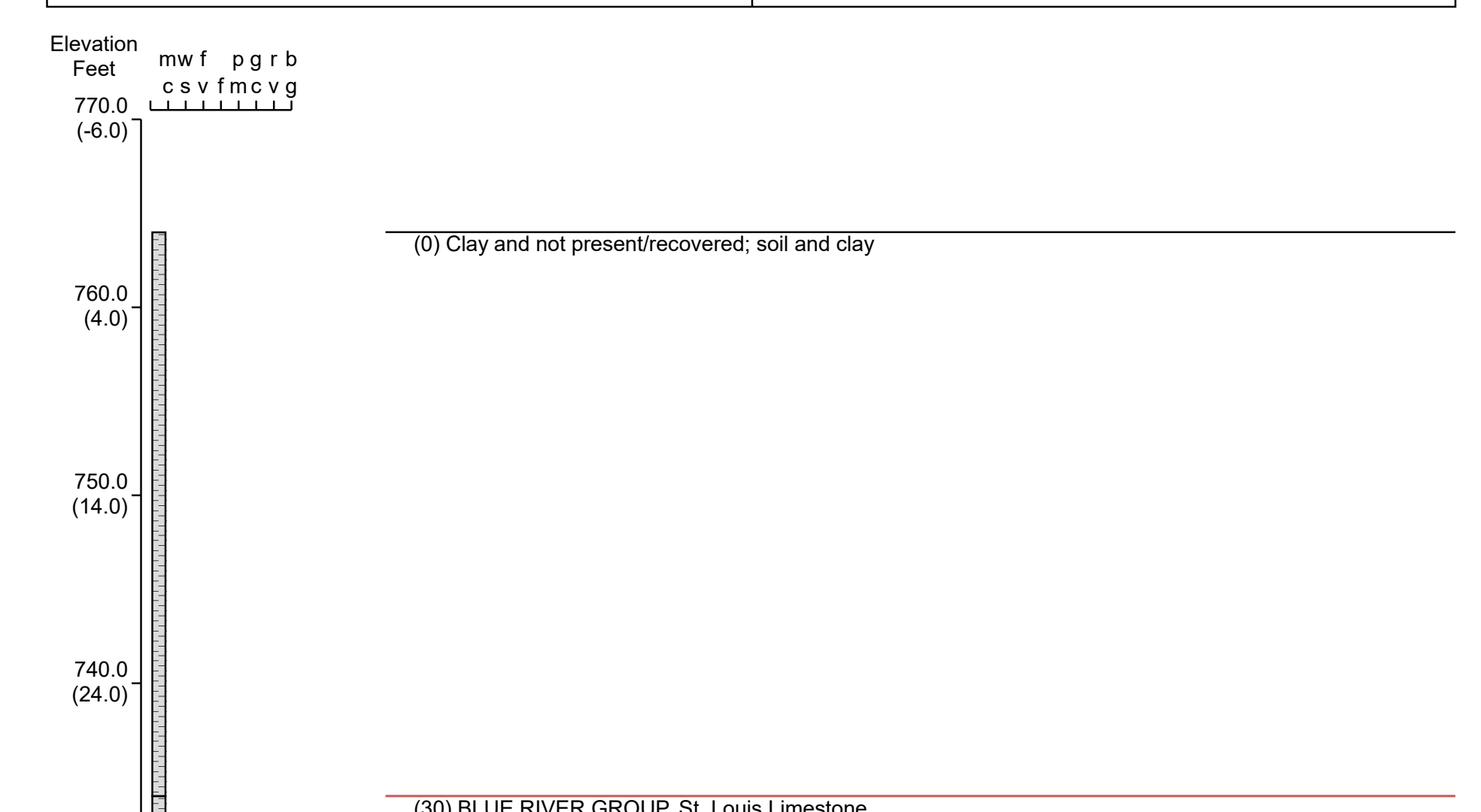

$\begin{array}{r}730.0 \\ (34.0) \\ \hline\end{array}$

Cllay; clasts at basal contact.

(31.8) Massive lime mudstone, irregular basal contact, thin wackestone zones at the top and base of the

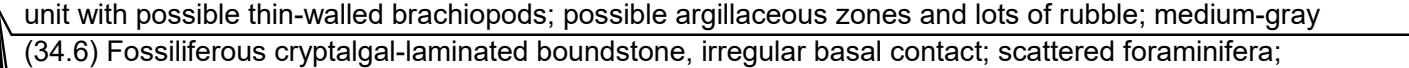
distinct algaal lamination: medium-oray with hight-tannish-oray void filling (micrite)

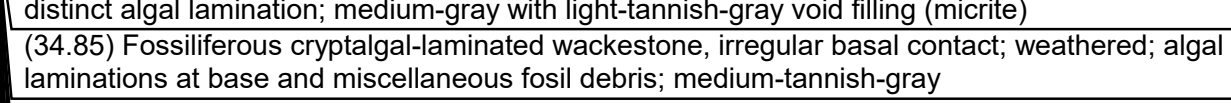

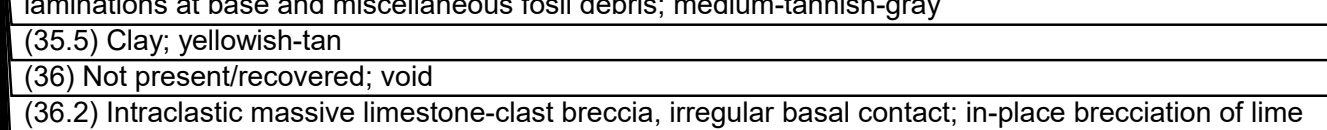
mudstone; light-annish-gray
(36.6) Horizontal-bedded lime mudstone; thin horizontal laminations; tannish-brown to medium-brownish-

(3r.a)
gray
(3a)

(3r) Horizontal-bedded carbonaceous shale, gradational basal contact; thin horizontal laminations:
unidentifiable white specks; black

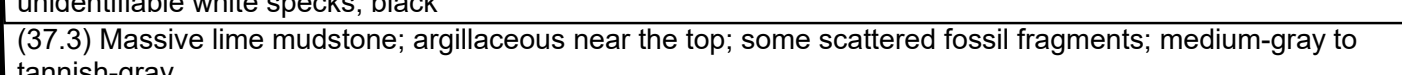

lannish-gray
$(38.65$ ) Massive siltstone; rubble zones; calcareous; clay-rich; tan
(40) Not presentrecovered

$(40)$ Not presentrecovered
$(40.6)$ Fossiliferous horizzontal-bedded wackestone; miscellaneous fossil debris throuhgout; clay-rich;

medium-brownish-gray
(41.1) Not presentrecovered; lime mudstone rubble
(42) Not presentrecovered void

$\begin{array}{r}700.0 \\ (64.0) \\ \hline\end{array}$

(4) Bioturbated horizontal-bedded argillaceous limestone; horizontal bitoturbation; medium-brownish-

(45.5) Not tresenentrecevorerera; rubble
(48) Bioturbated massive sandstone; clay-rich; calcareouss; prominent oval bioturbation; tannish-gray

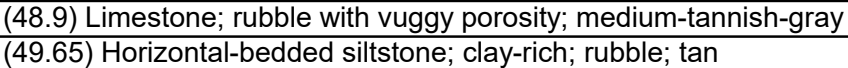

$\underset{(74.0)}{690.0}$

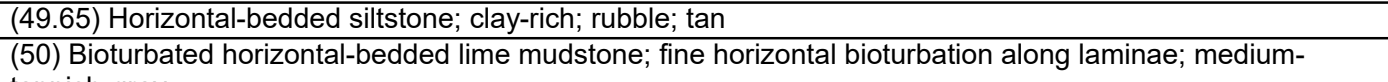

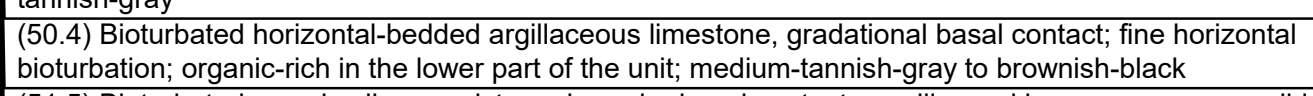

(51.5) Bioturbated massiviv lime mudstone

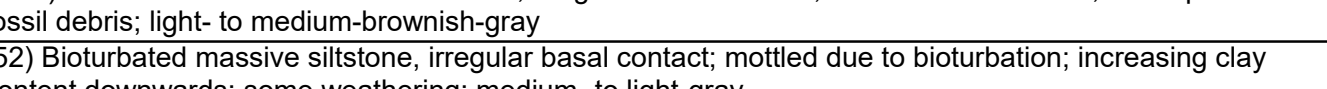

(53.8) Clay; calcareous toward the base; orangish-yellow to greenish-gray
(54.8) Bioturbated horizontal-bedded lime mudstone; bioturbation and clay content increase toward the

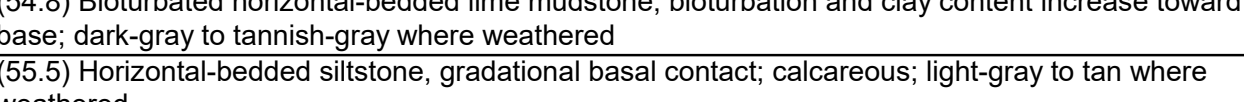

(55.7) Mottled and bioturbated horizontal-bedded lime mudstone; thin zones of fine fossil debris; medium-

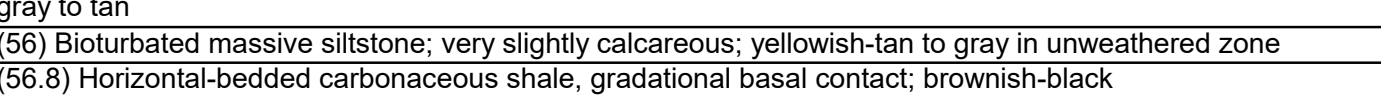

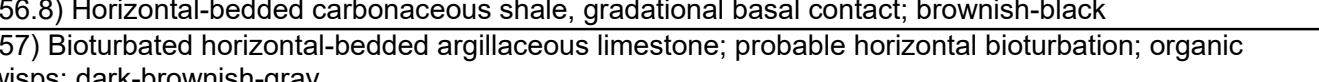

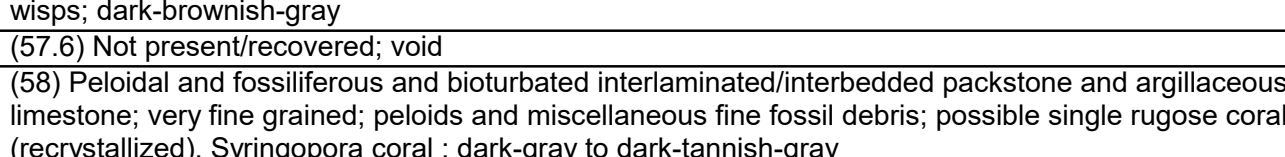

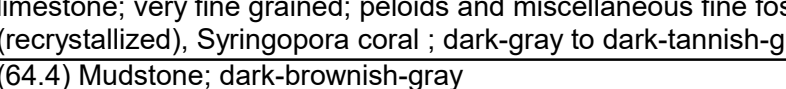

(64.45) Fossilifierous horizontal-bedded argillaceous limestone, gradational basal contact; organic wisps

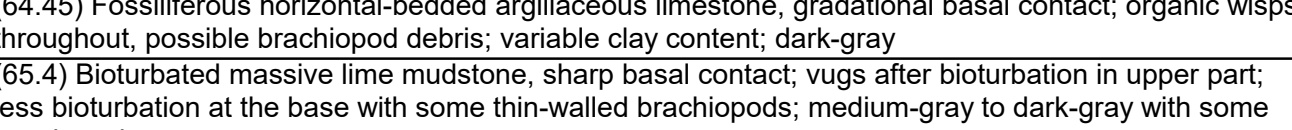

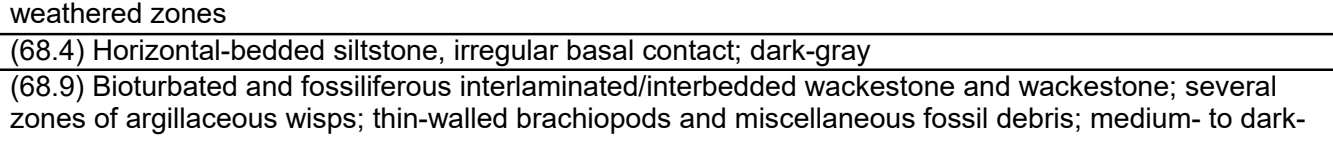

zones of argillaceous wisps; thin-walled brachiopods and miscellaneous fossil debris; medium-to dark-
gray

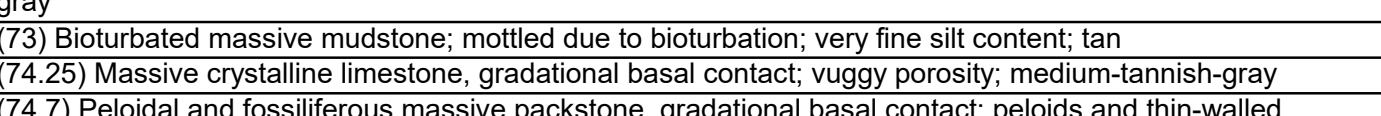

brachiopods; medium-tannish-gray
75 Massive lime mudstone and argillaceous limestone; medium-tannish-gray
75 g) Not resentrecovered' void with some lime mudstone rubble

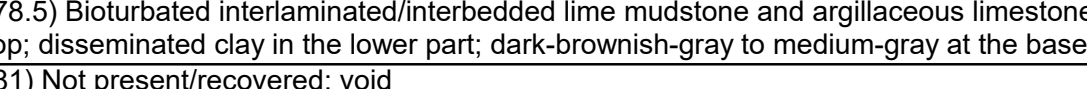

(B) 2) Horizontal-beddded lime mudstone, sharp basal contact; prominent horizontal bioturbation; light-

$\begin{array}{r}630.0 \\ (134.0) \\ \hline\end{array}$

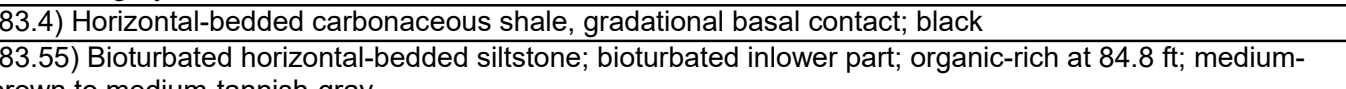

(85.4) Bioturbateded horizontalab-bedded lime mudstone, gradational basal contact; minor bioturbation;

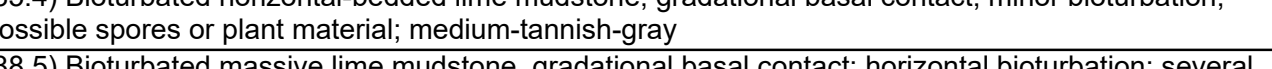

argiliaceous zones; medium-dark-gray

gray
92.3) Horizontal-bedded sandstone, gradational basal contact, finely laminated; disturbed at the base;

Lower very fine grained sand; mediut-tannish-gray
93.9. Biotorurbated massive sandstone, sharp basal contact; churned due to bioturbation; medium-

greenish-gray
(97.05) SANDERS GROUP, Salem Limestone
Fosiliferous and bioturbated horizontal-bedded packstone, irregular basal contact, miscellaneous foss

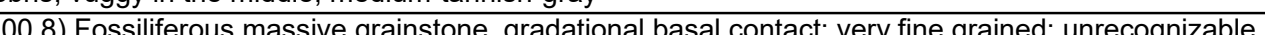

(10)

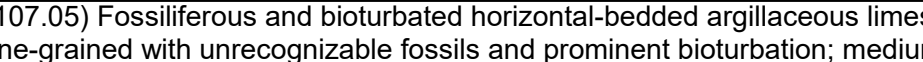

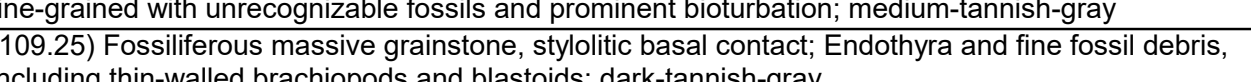

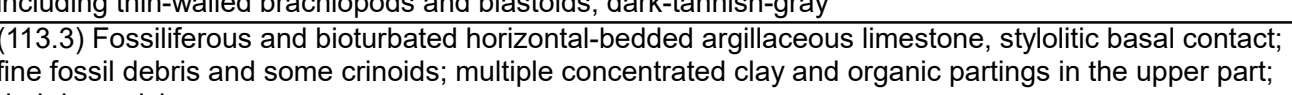

ine fossil debris and some crinoids; multiple concentrated clay and organic partings in the upper part;
darab-rowinish-gray
(114

dark-brownish-gray
(11448) Fossifferous massive grainstone, styllititic basal contact, fine fossil debris; medium- to dark-
annish-gray

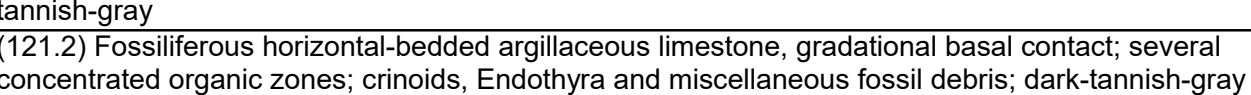

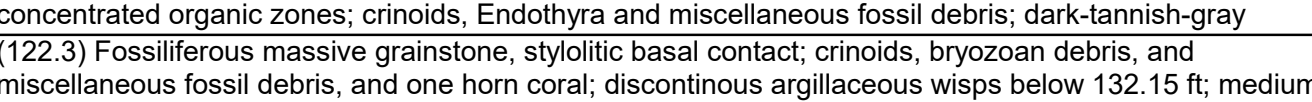

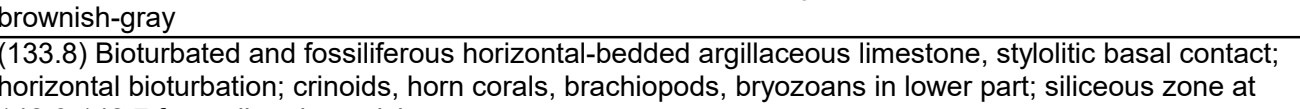

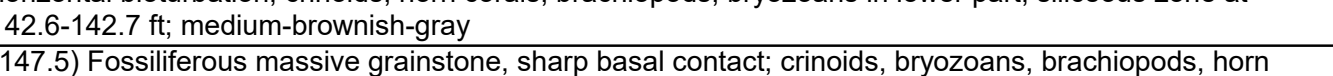

coral, and fine fossil debris; medium- to dark-brownish-gray
(150.65) Horizontal-bedded carbonaceous shale, sharp basal contact; calcareous; black

(150.7) Fosilififrous massive grainstone, gradatational basal contact, fine crinoids, and miscellaneous
(15sil debris

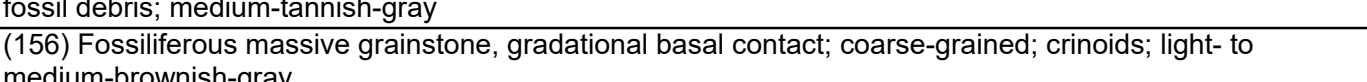

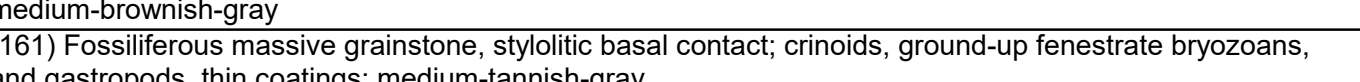

and gastropods, thin coatings; medium-tannish-gray
$(181.55)$ Horizontal-bedded siltstone, irregular basal contact; dark-greenish-gray

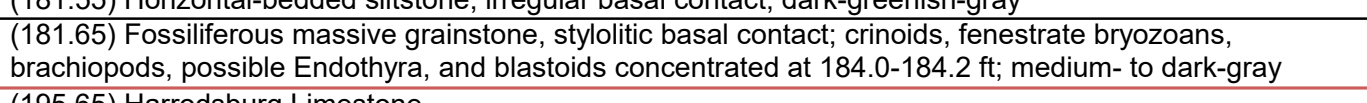
(195.65) Harrodsbbror Limestone
Fossiliferous horizontal-bedded packstone; variable mud contents; occasional argillaceous zone;
crinoids, fenestrate bryozzoans, brachiopods, small black grains; medium-gray 


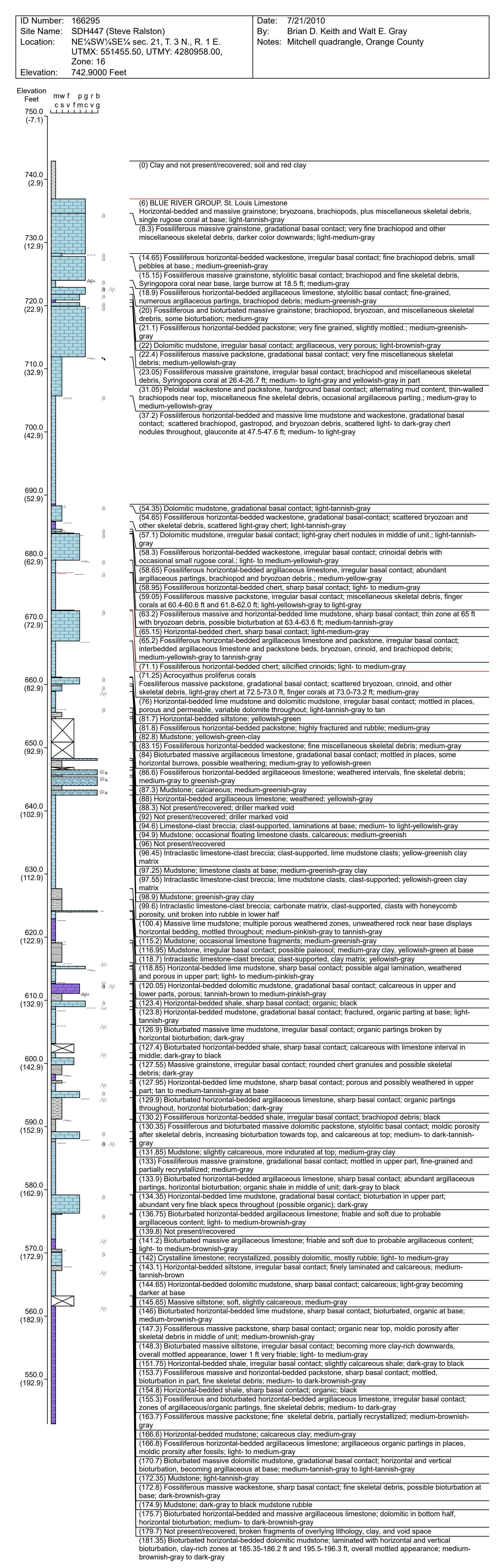




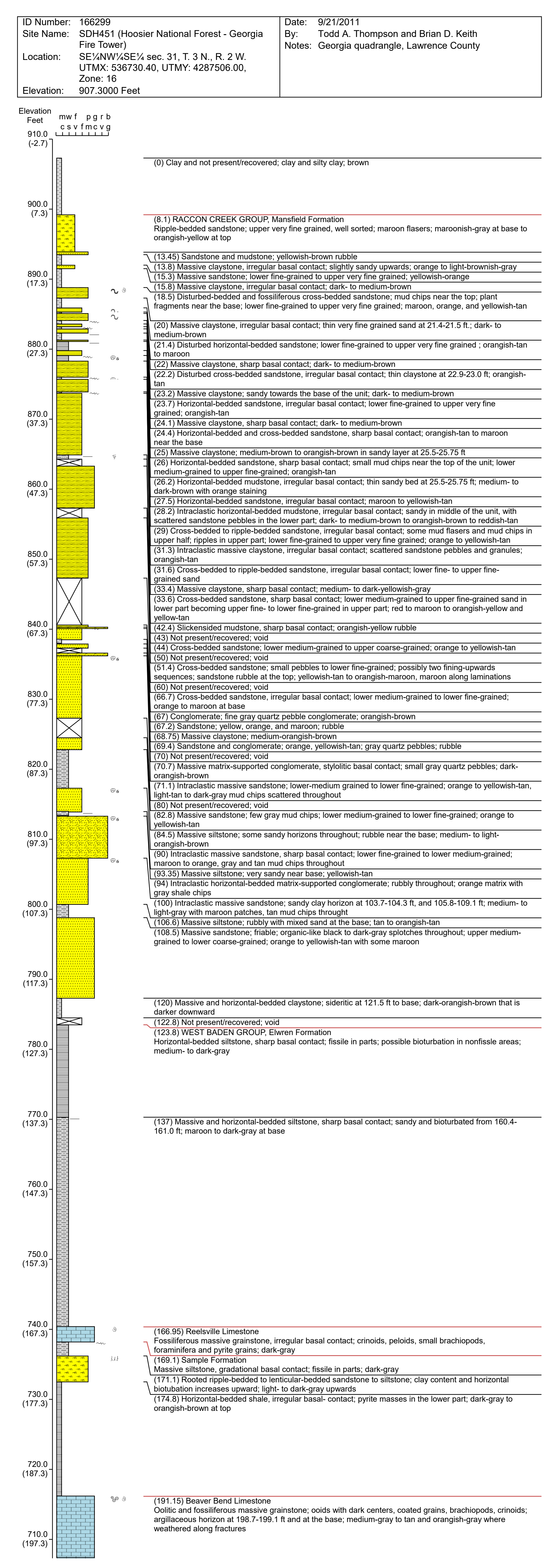




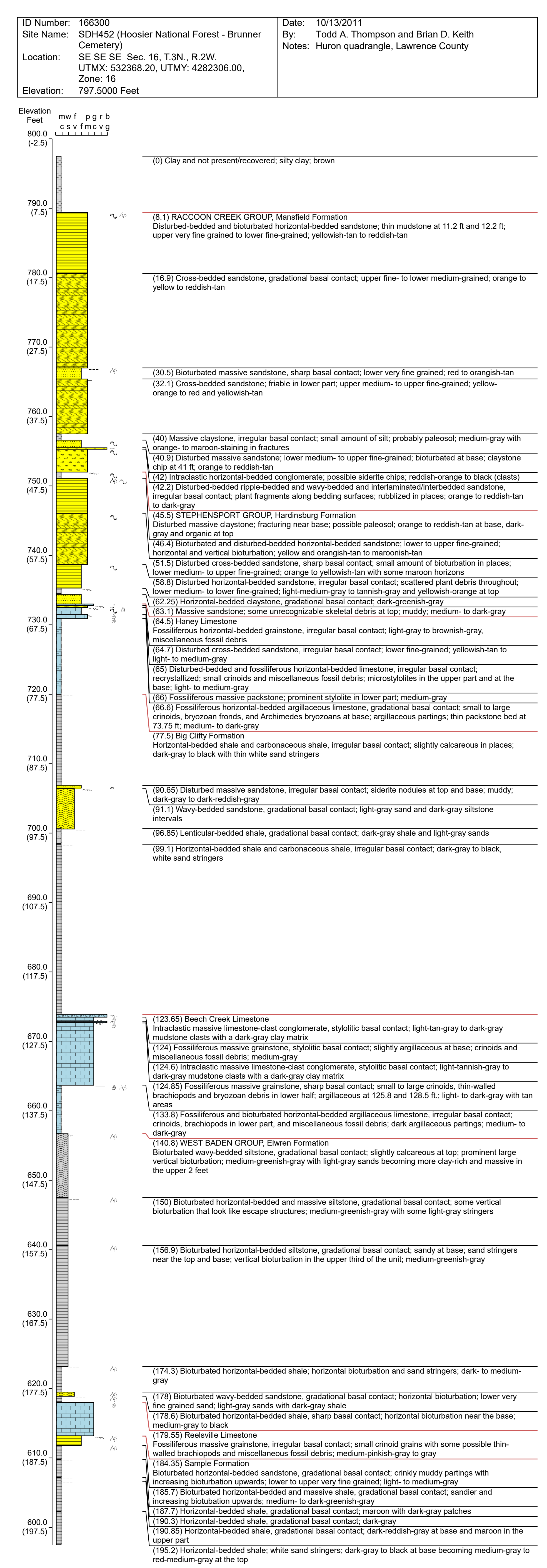




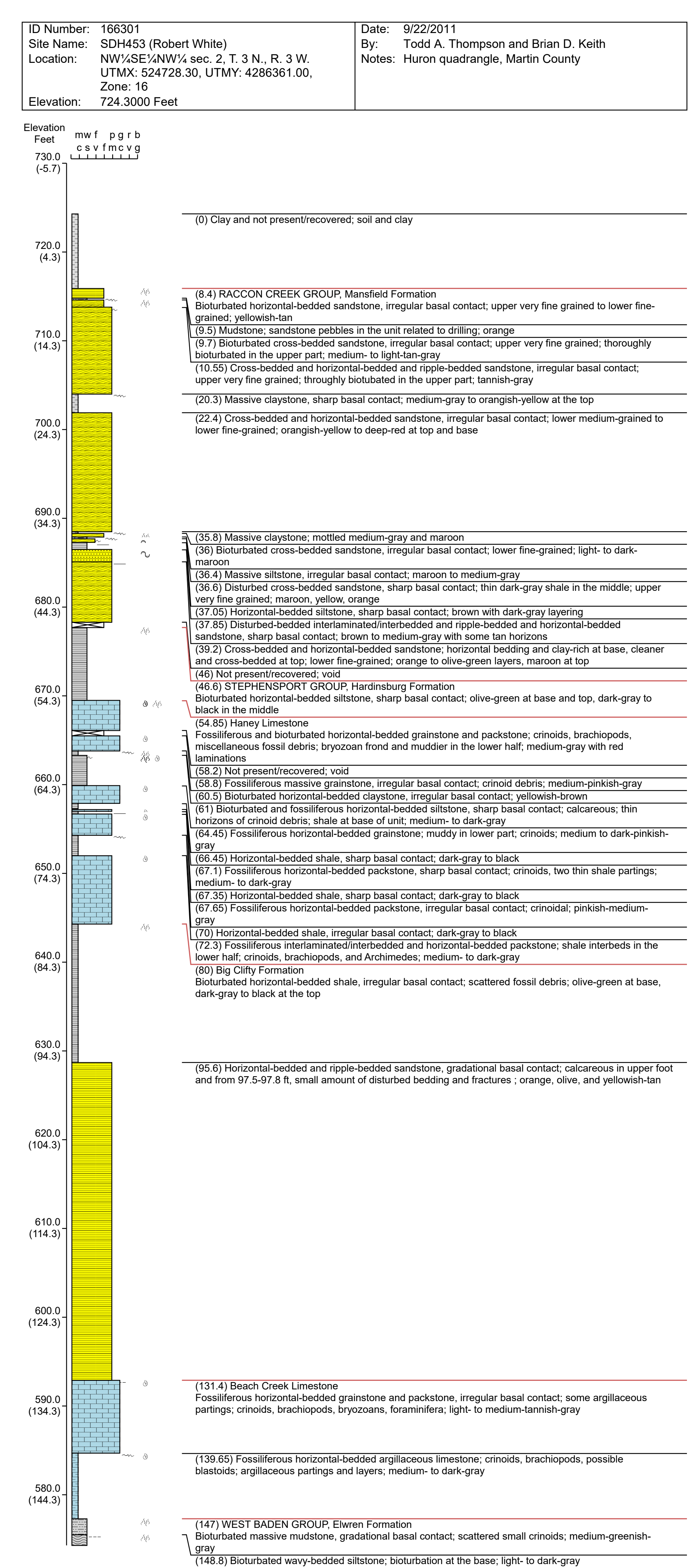




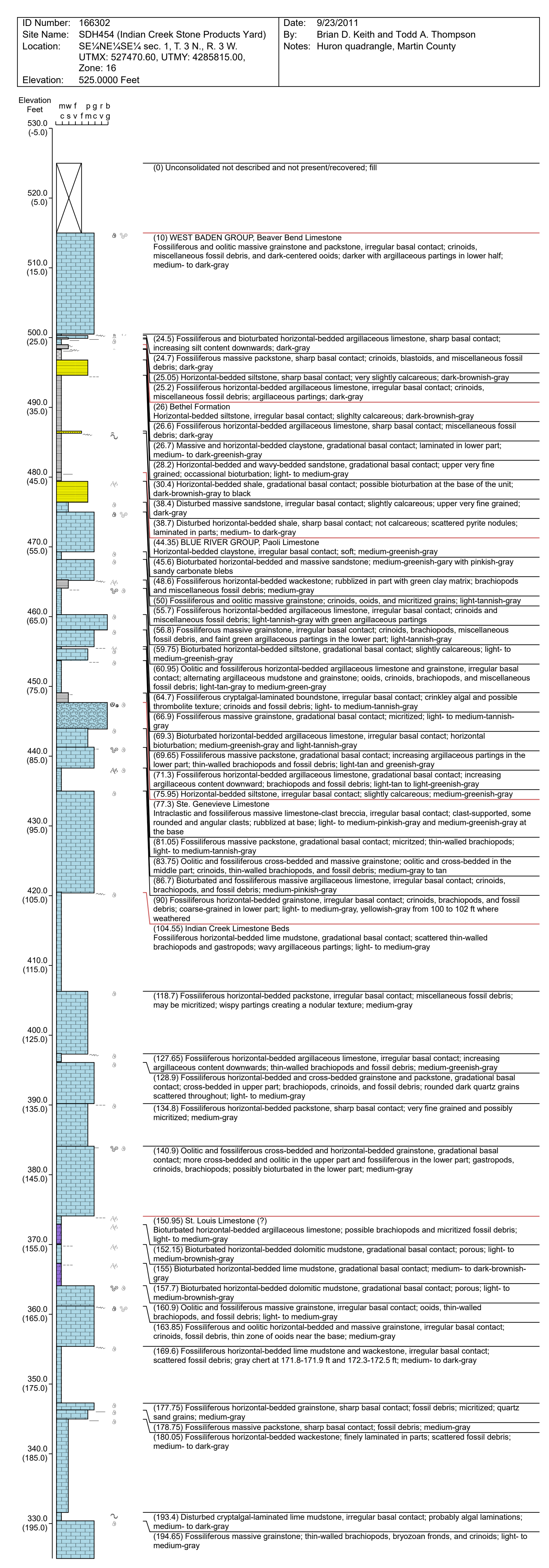




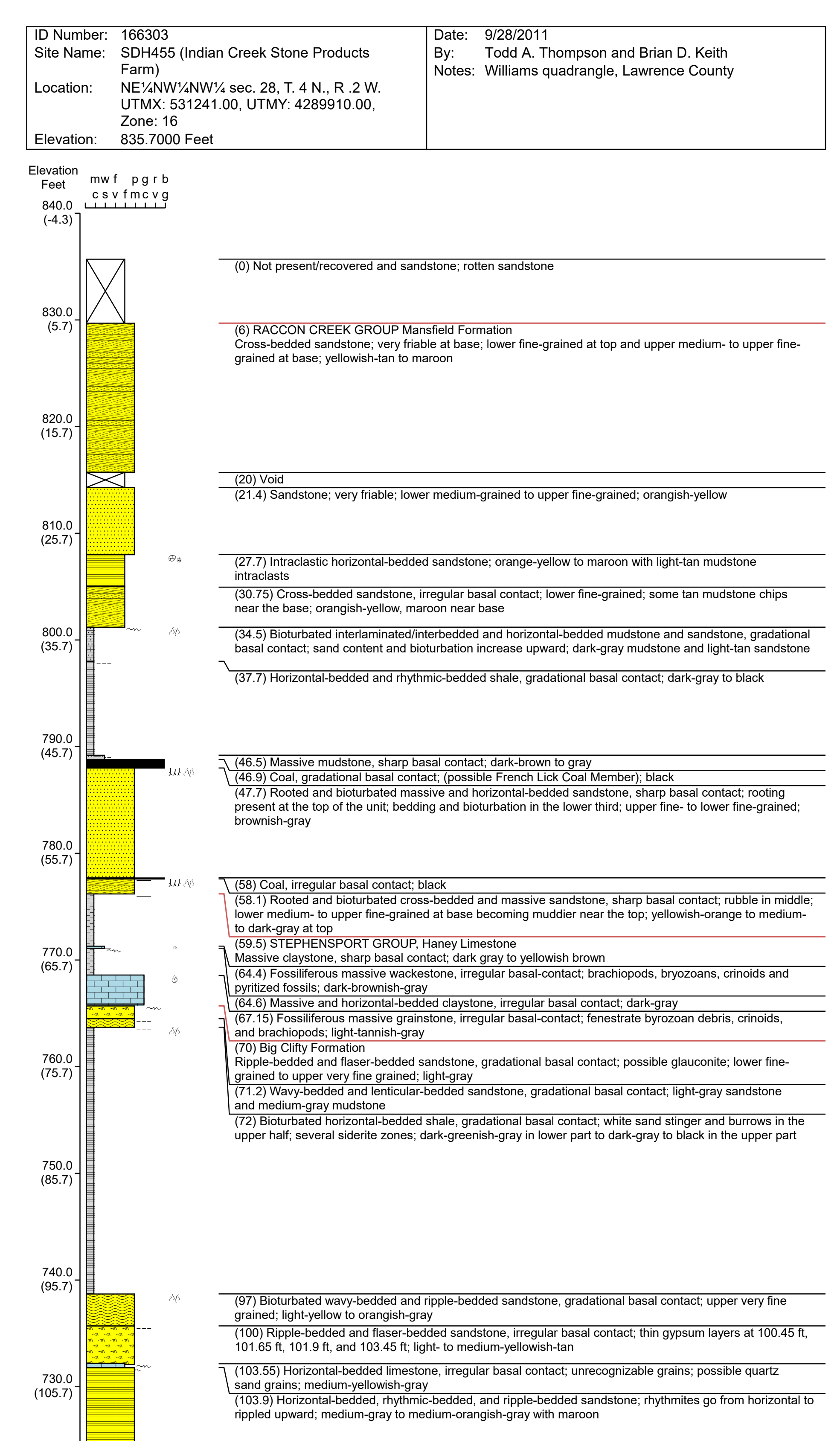

720.0
$(115.7)$

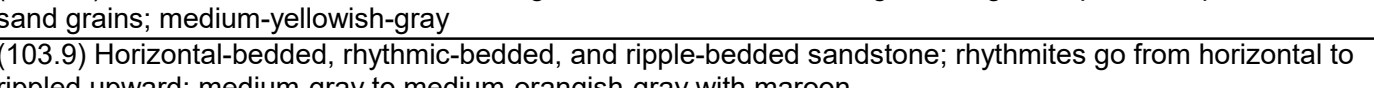




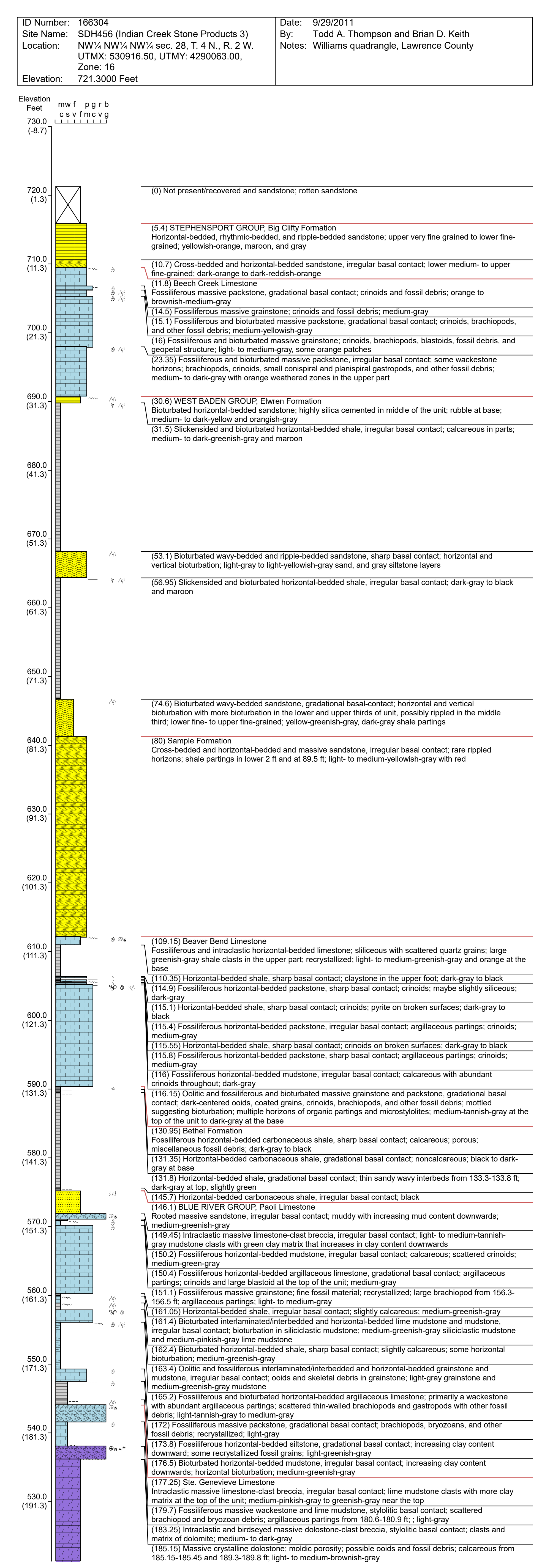




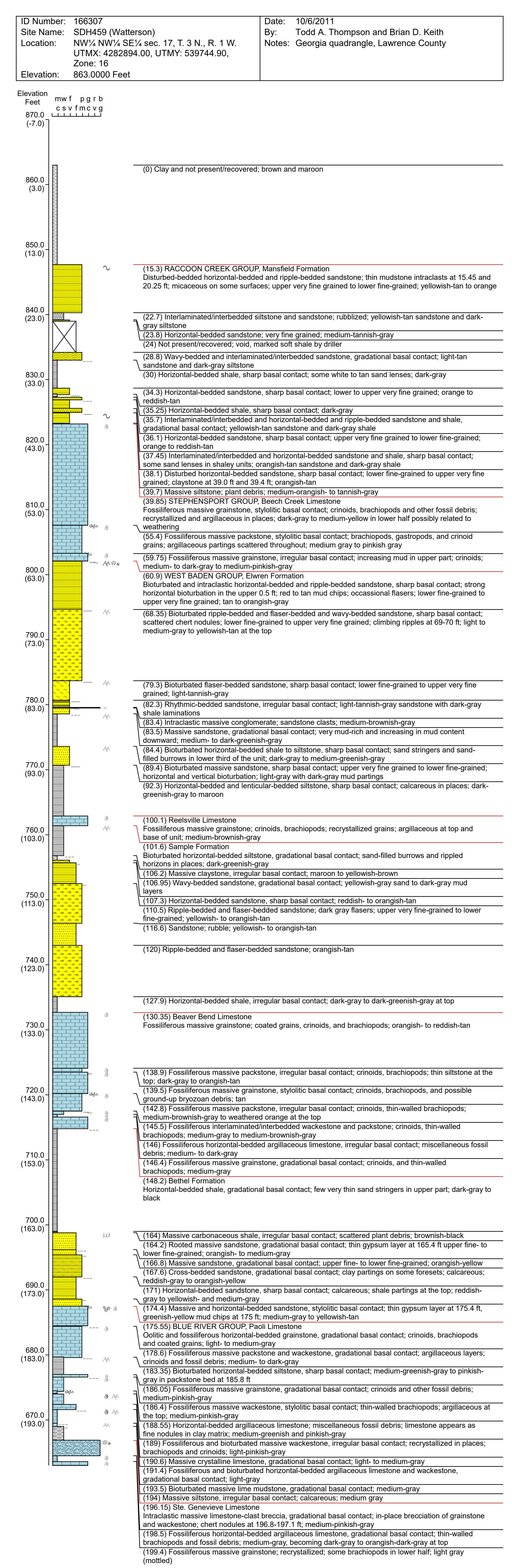




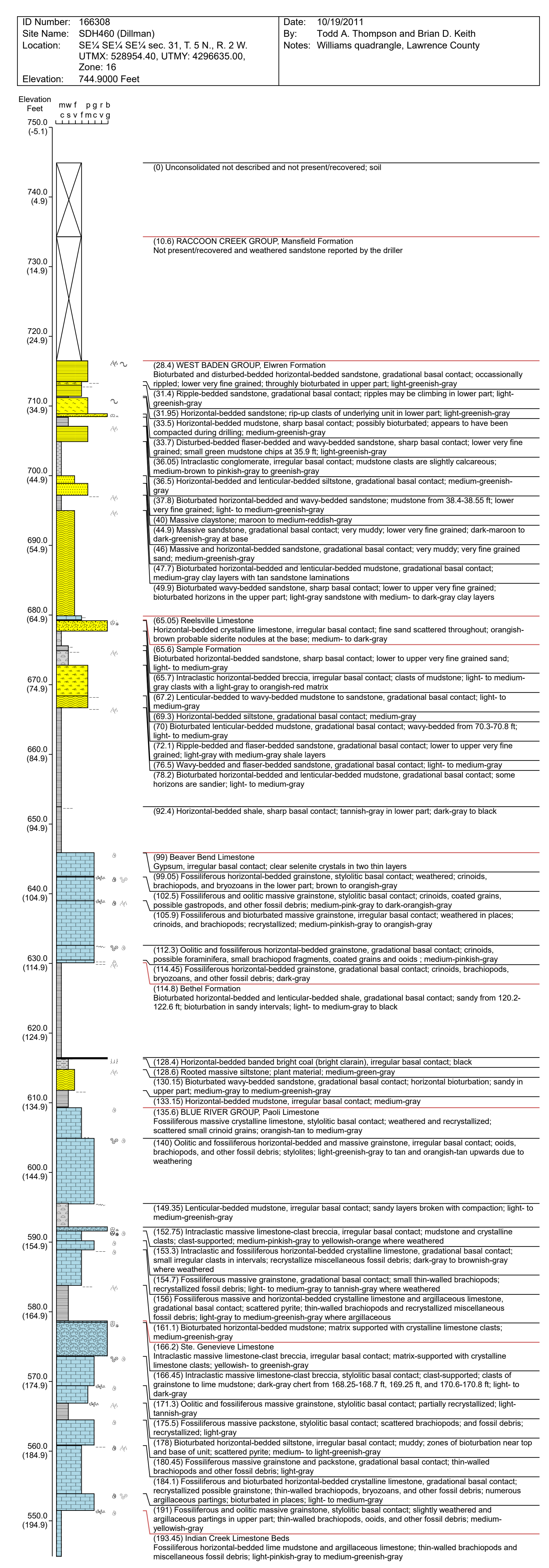




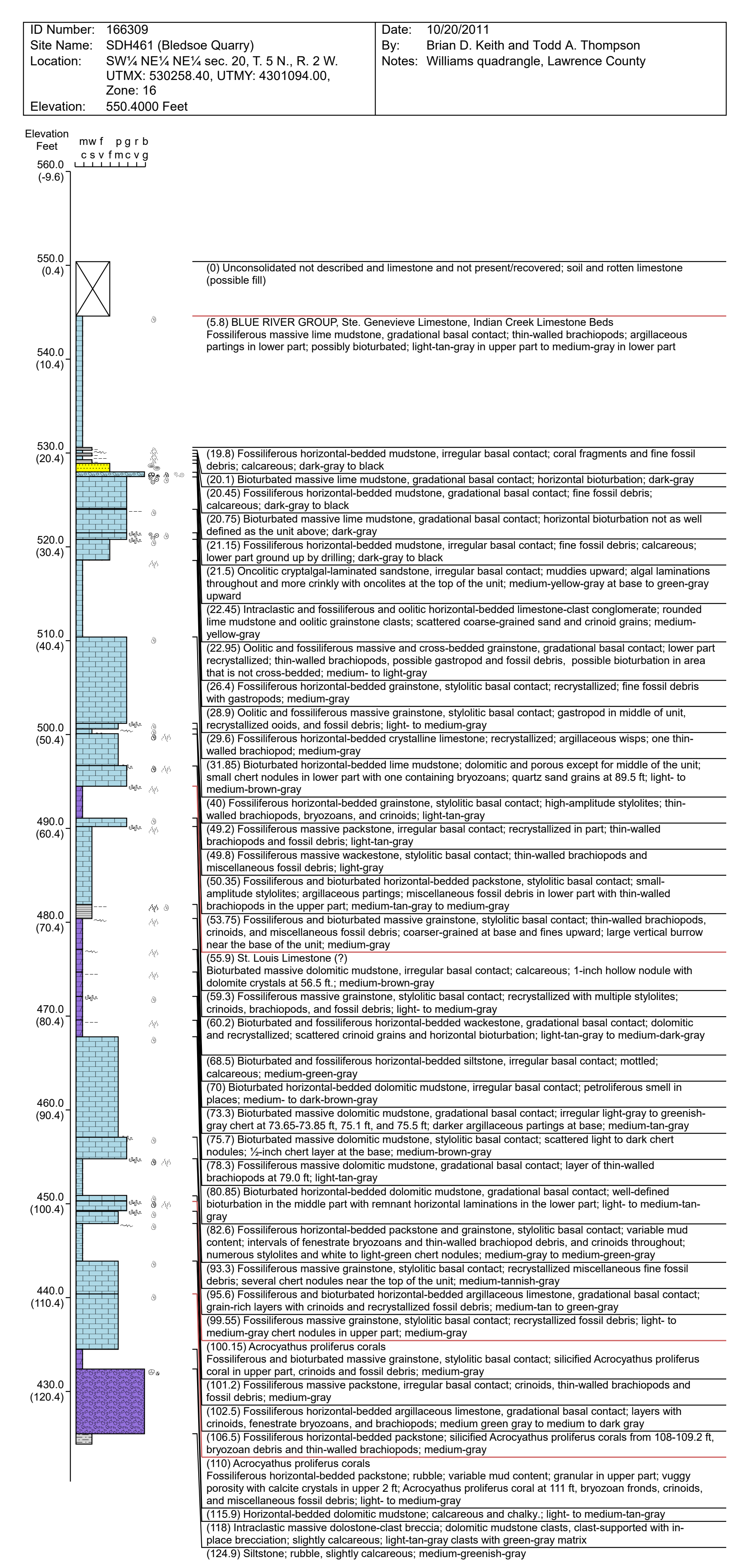





\begin{tabular}{|c|c|c|}
\hline $\begin{array}{l}\text { Do Number: } \\
\text { Site anam: } \\
\text { Location: } \\
\\
\text { Elevation: }\end{array}$ & 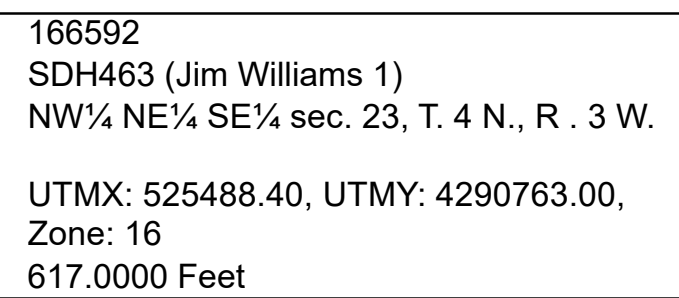 & 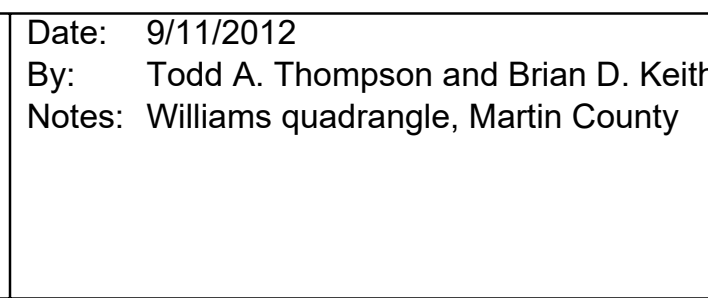 \\
\hline
\end{tabular}

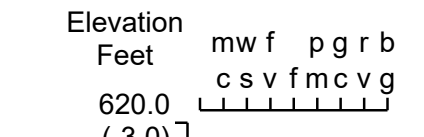

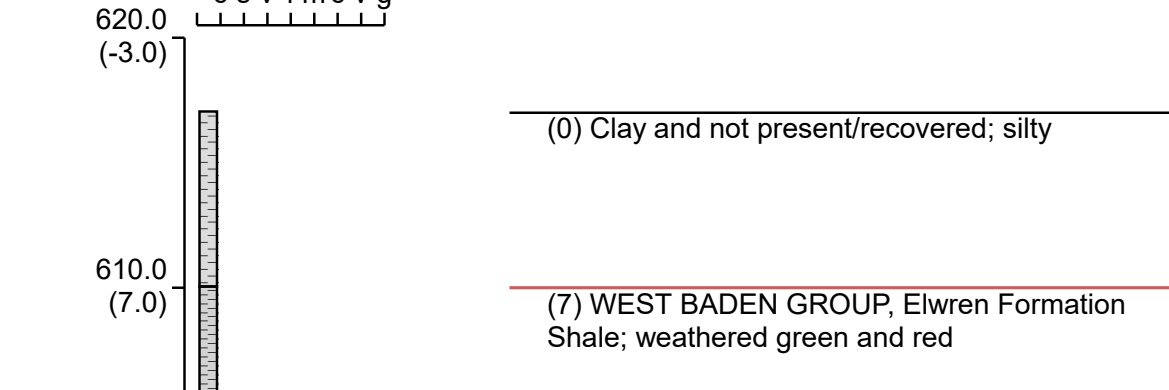
600.0
$(17.0)$
(20) Siltstone; light-brown
227.0
(27) Sandstone, rotten
(40) Bioturbated horizontal-bedded shale, gradational basal contact; medium-to dark-gray to maroonish- gray

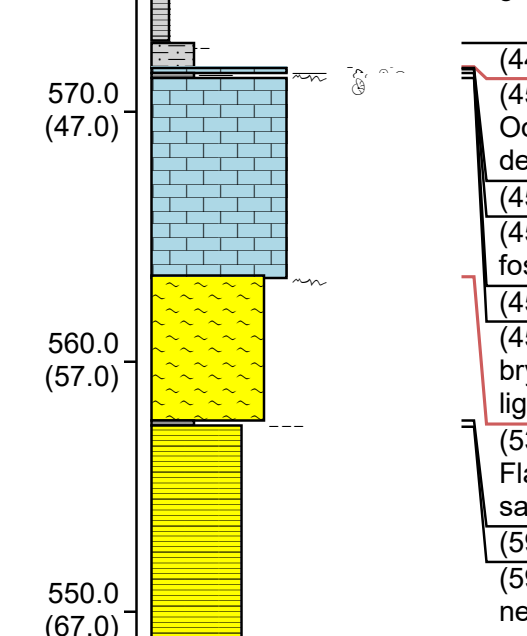

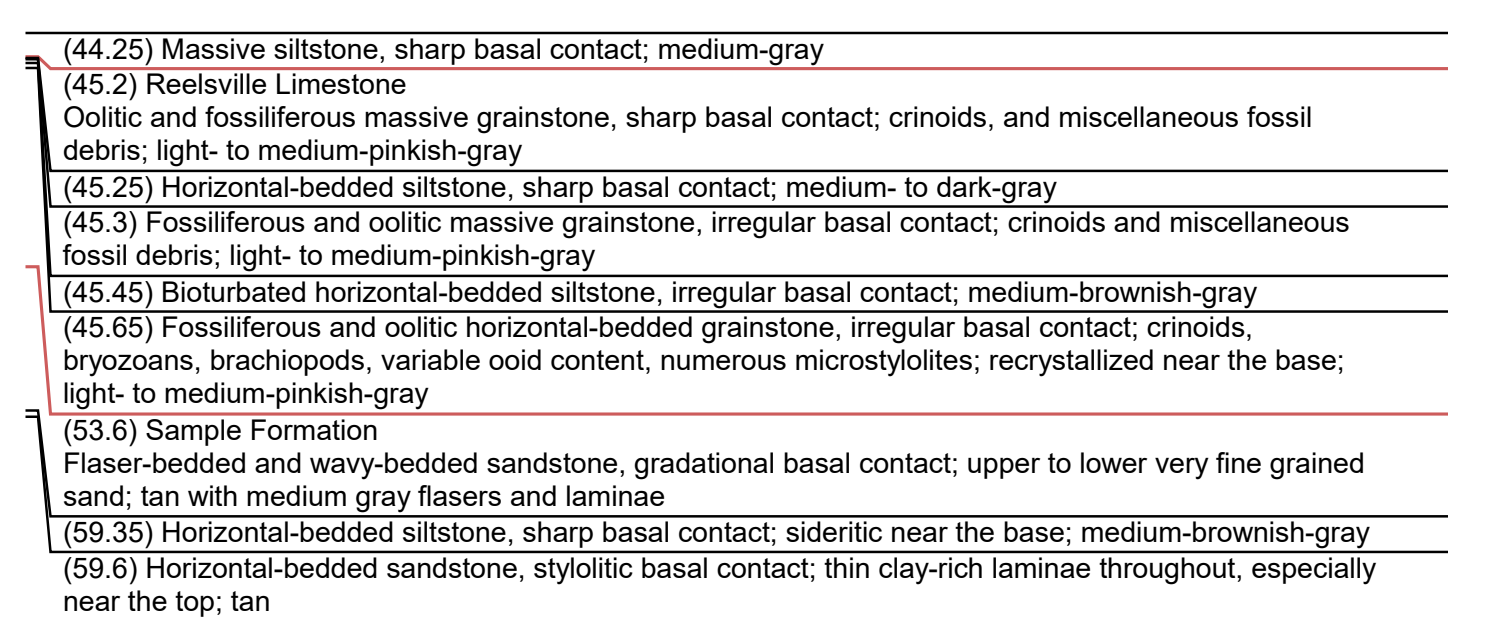

(69.45) Beaver Bend Limestone

Oolitic and fossiliferorous massivive grainstone, recrystallized with micritized ooids; ilight- to medium-gray (70.2) Fimssiliferous massive grainstone, gradadtional basal contact; crinoids, brachiopods; increasing

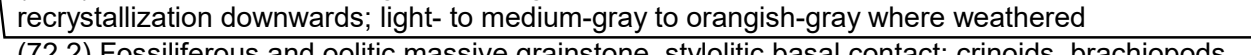

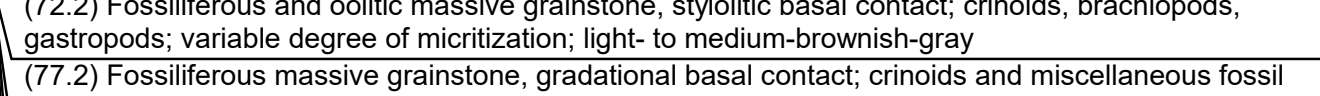

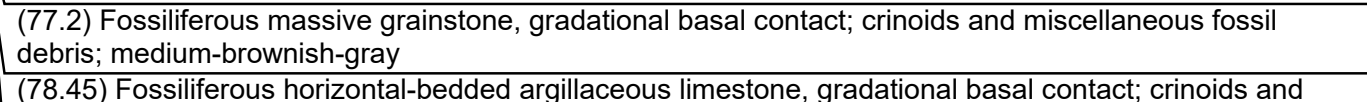

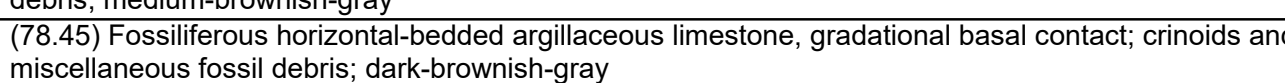
(78.9) Fossiliferous massive grainstone, stylolitic basal contact; crinoids, gastropods, brachiopods;

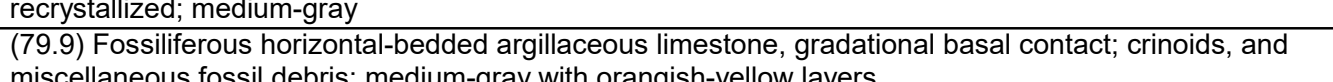

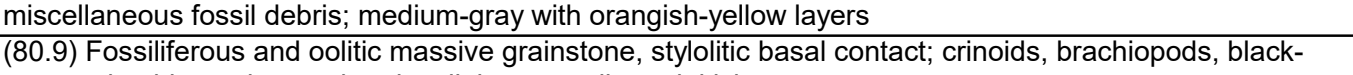
\begin{tabular}{l} 
eentered ooids, and coated grains; ilight- to medium-pinkish-gray \\
\hline 84.7$)$ Horizontal-bedded limestone, irregular basal contact; abundant quartz grains; miscellaneous fossil
\end{tabular}

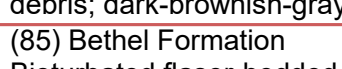

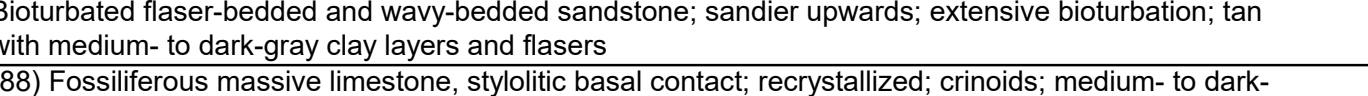
$\frac{\text { gray }}{(88.3) \text { Fossiliferous horizontal-bedded argillaceous limestone, styllitic basal contact; crinoids and }}$

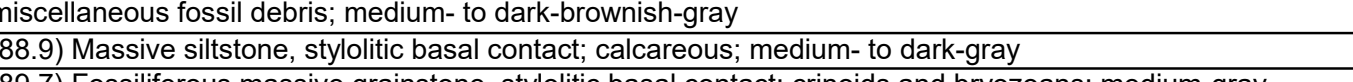
作 argillaceous limestones; miscellaneous fossil debris; ight-to medium-gray
(93.6) Bioturbated horizontal-beddded shale, gradational basal contact; horizontal bioturbation; dark-gray to

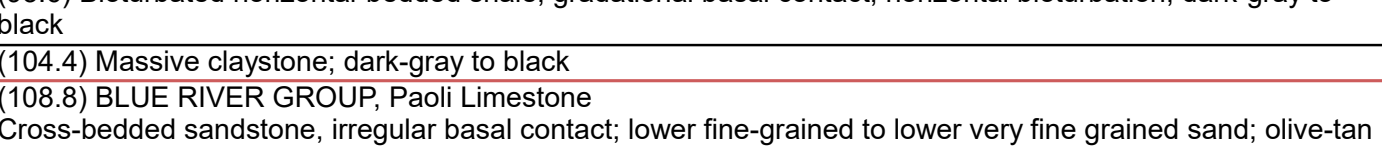
(110.5) Fossiliferous massive wackestone; miscellaneous fossil debris; recrystallized and silicified at the

(op; medium-greenish-gray $(110.8)$ Rooted massive sandstone, irregular basal contact; upper fine-grained to upper very fine grained Sand with clayy medium-greenish-gray
(111.4) Fossilfifrous massive wackestone; thin-walled brachiopods, miscellaneous fossil debris;

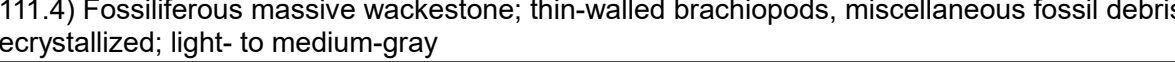

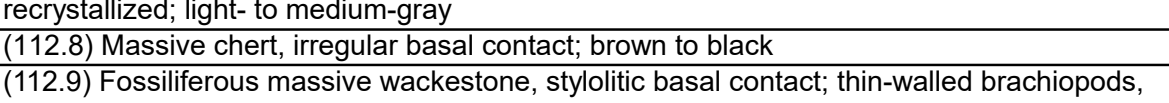

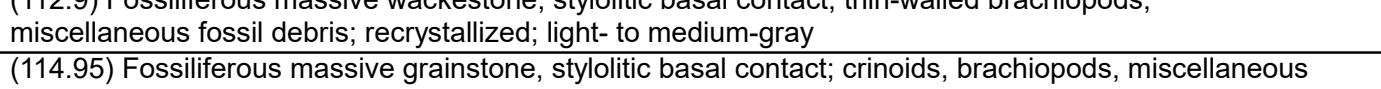

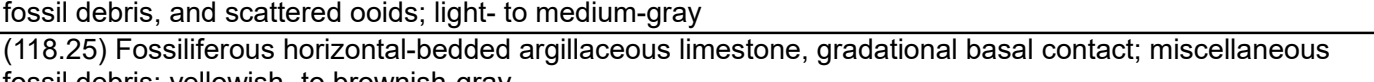
(118.4) Fossilififrous massive grainstone, stylolitic basal contact; crinoids and miscellaneous fossil debris; recrystallized; ilight- to medium-yellowish-gray
(119.3) Oolitic and fossififerous horizontal-hedded grainstone: fossiliferous at the base becoming oolitic

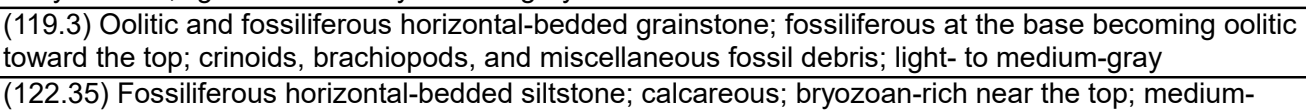

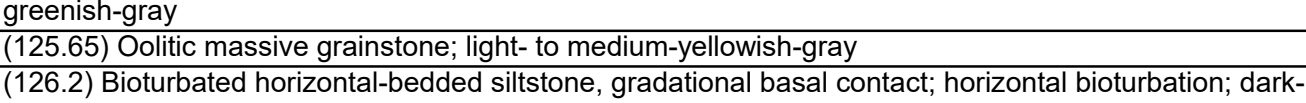

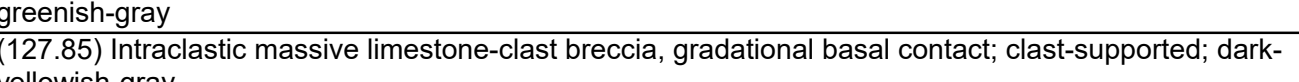

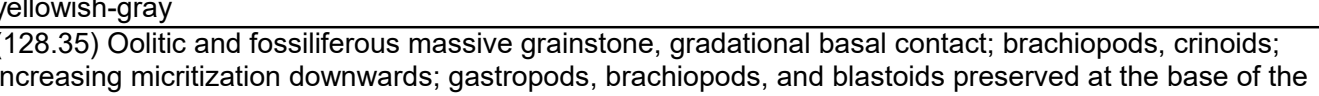
uniti, light- to medium-gray
131.11) Bioturbated massive lime mudstone; thin-walled brachiopods; horizontal biturbation: light-

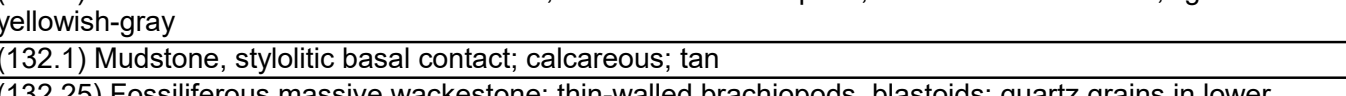
tal-bedded silitstone; horizontal and vertical bioturbation; greenish-gray to

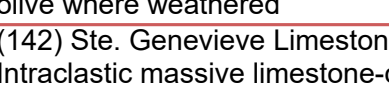

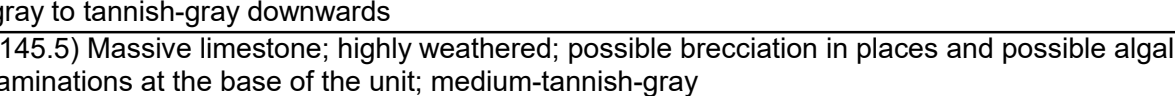




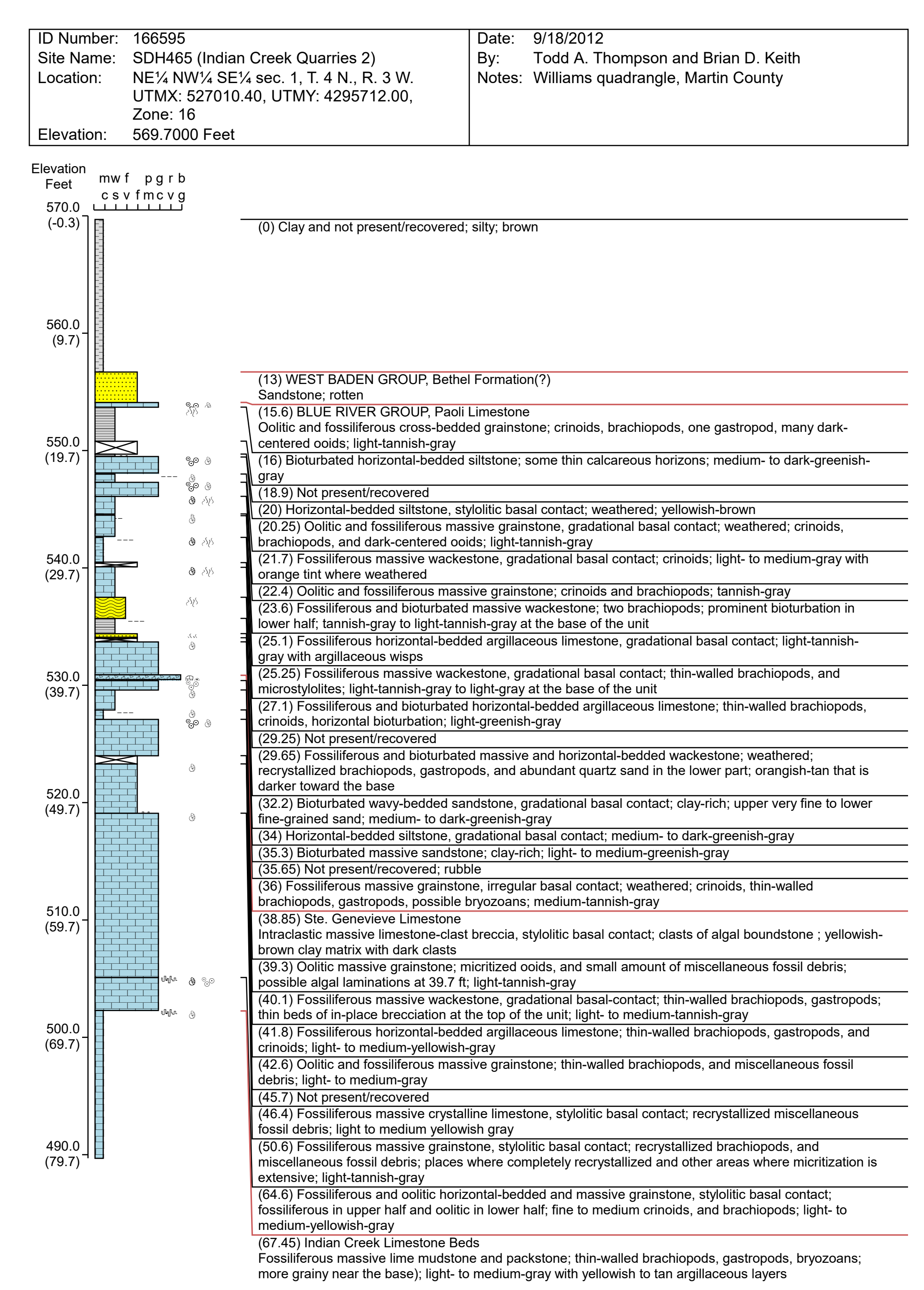




\begin{tabular}{|c|c|c|}
\hline \begin{tabular}{|l} 
IN N Number: \\
Site Name: \\
Location:
\end{tabular} & 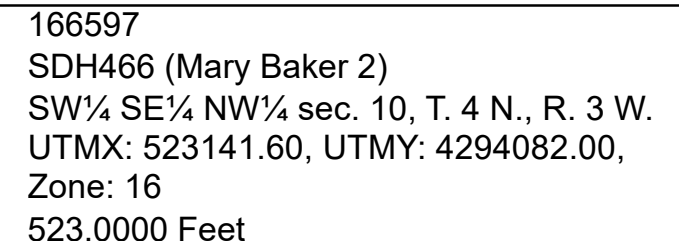 & 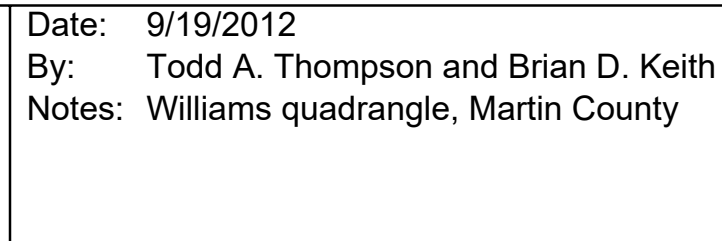 \\
\hline
\end{tabular}

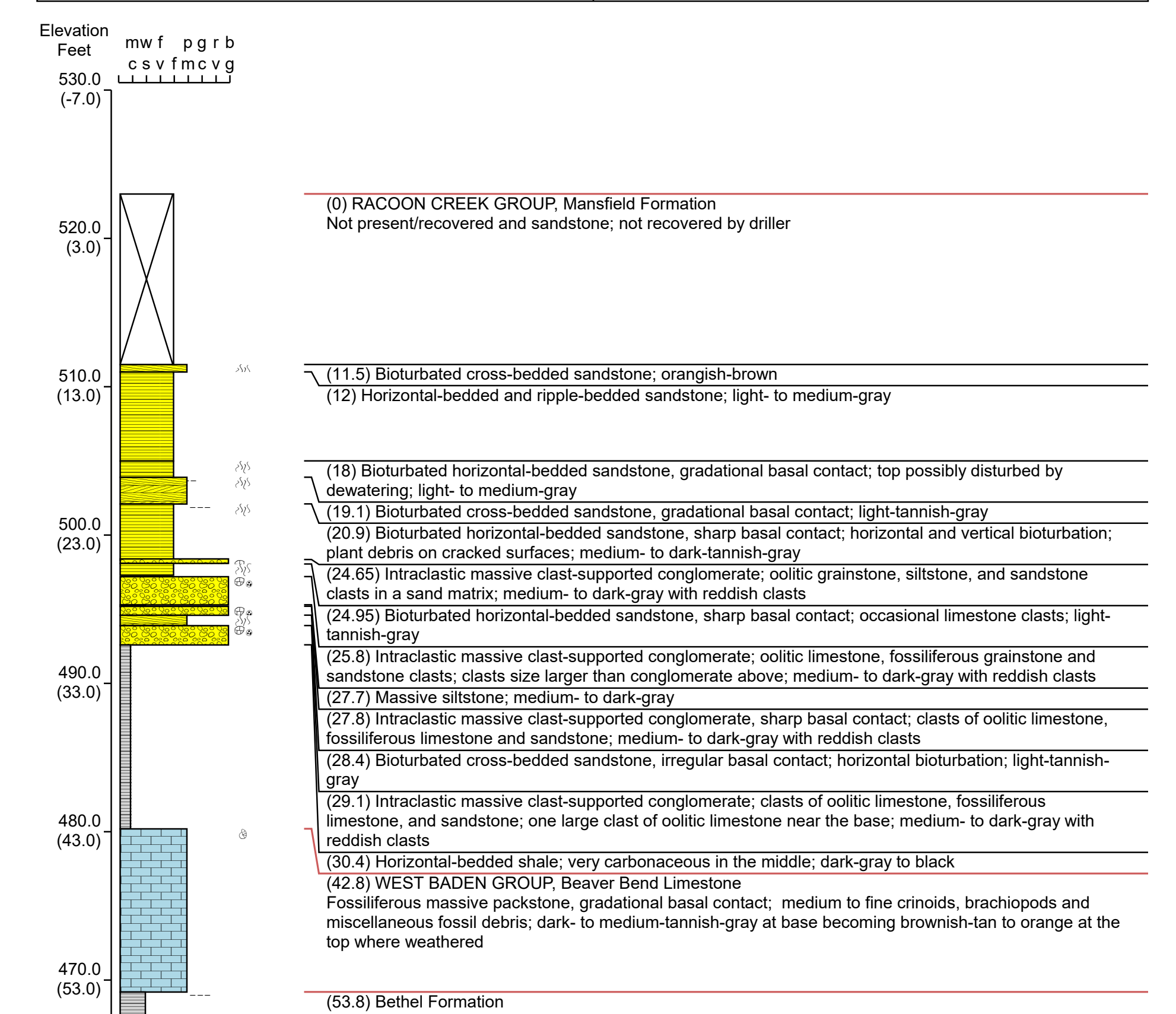

(53.8) Bethel Formation
Horizontal-beddded siltstone, irregular basal contact; dark-gray to black

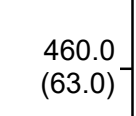

(61.55) Wavy-bedded and lenticular-bedded sandstone, gradational basal contact, variable sand and clay

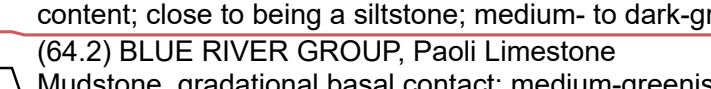

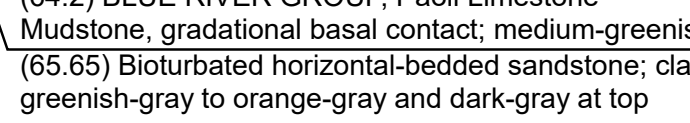

$\underset{\substack{4500 \\(300)}}{4}$

(170

(70.6) Intraclastic massive limestone-clast breccia; rubble in lower part; clast-supported packstone,
medium--inkish-gray at top to yellowish-green at base

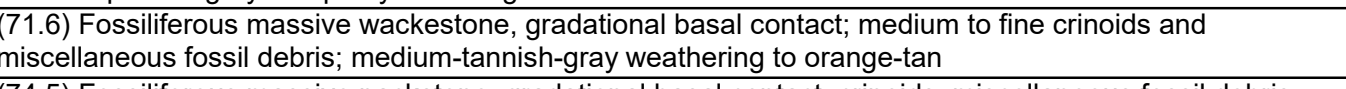

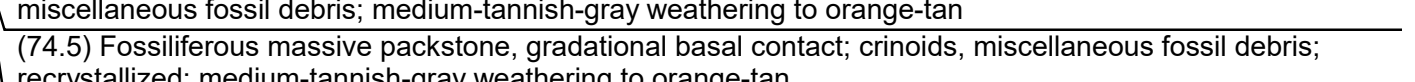
recrystallizedi medium-tannish-gray weathering to orange-tan
$(75.6)$ Fossilfifrous and oolitic massive grainstone, stylylitic basal contact; weathered; recrystallizized; fine Crinoids and miscellaneous fossil debris; medium-tannish-gray
(77.5) Fossiliferous and oolitic horizontal-bedded grainstone, irregular basal contact; o oids concentrated in

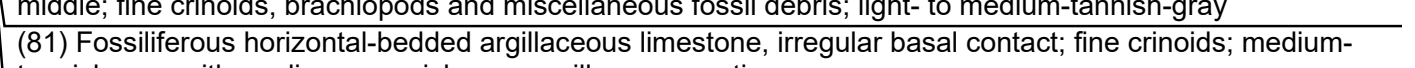
tannish-gray with medium-greenish-gray argillacoous partings

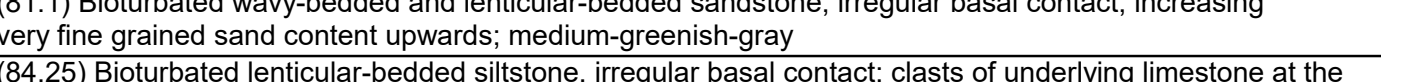
(84.25) Bioturbatede lenticular-bedded siltstone, irregular basal contact; clasts of underlying limestone at the
base; medium-greenish-gray

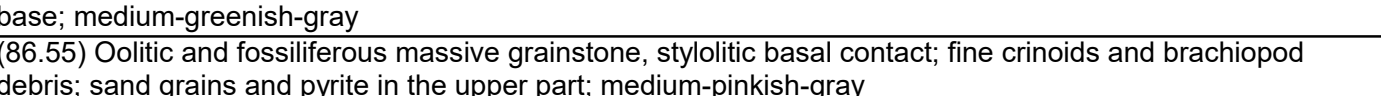

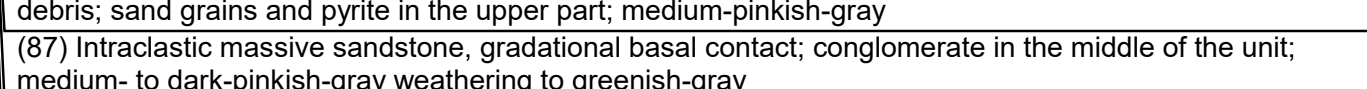

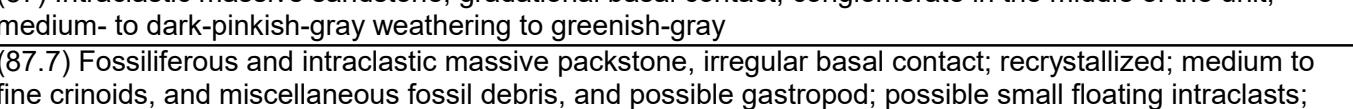
\begin{tabular}{l} 
medium-pinkish-gray to orangish-tan where weathered \\
\hline (89.7) Intraclastic massive limeston--clast breccia; ime mudstone brecciated in places; clast-supported;
\end{tabular} light-tannich-gray weathering to orangish-tan
(92.8) Fossiliferous and bioturbated horizontal-bedded argillaceous limestone, gradational basal contact;

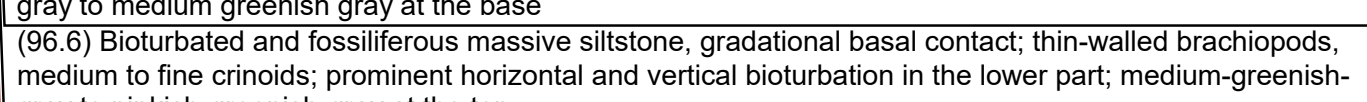

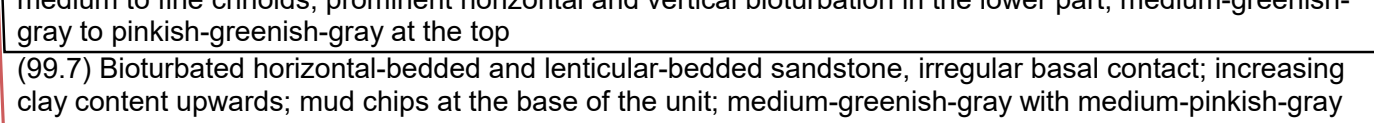
(licker sandstones
(105.2) Ste Genevieve Lines 105.2) Ste. Genevieve Limestone
ntraclastic massive limestone-clast breccia; lime mudstone clasts; clast-supported; rubble in lower part:

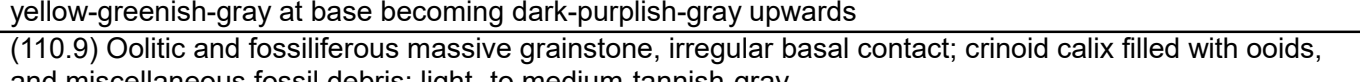
\begin{tabular}{l} 
and miscellaneous fossil debris; ilight-to medium-tannish-gray \\
\hline 111.8 F
\end{tabular} doww ards; brachiopods, gastropods and miscellaneous fossil debris; large-scale vertical bituturbation; i light-
gray gray
(118.4) Fossiliferous horizontal-bedded dolomitic packstone, gradational basal contact; brachiopods and
miscellaneous fossil debrisis variably calcareous: i ight-tannish-gray

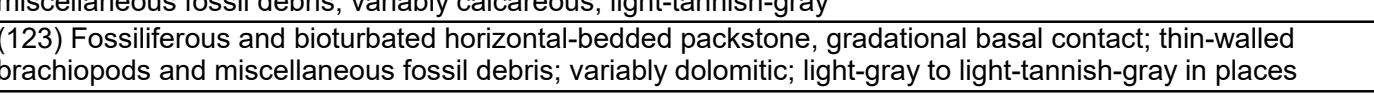

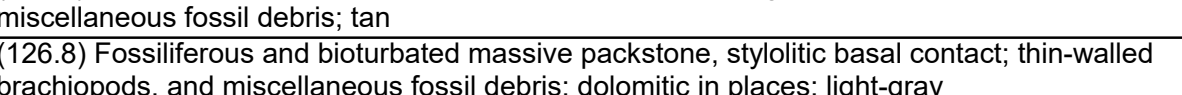

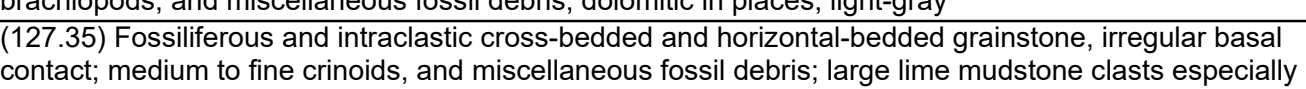

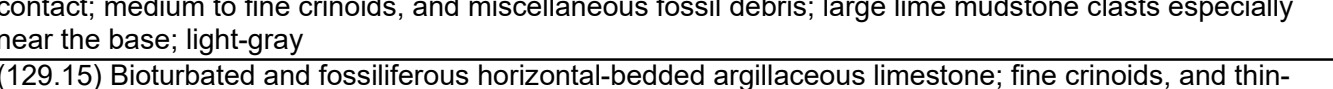

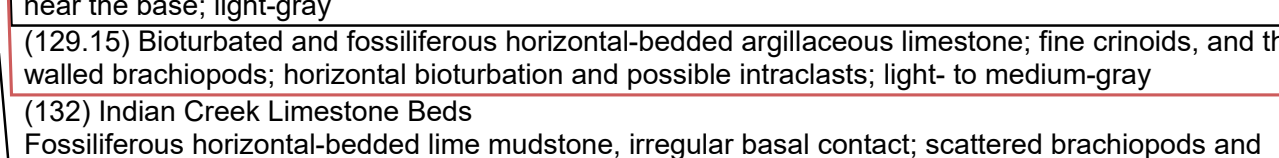

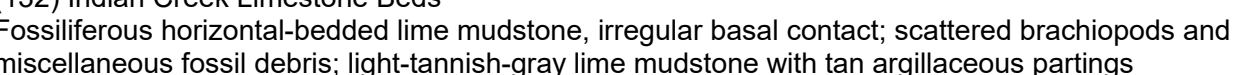

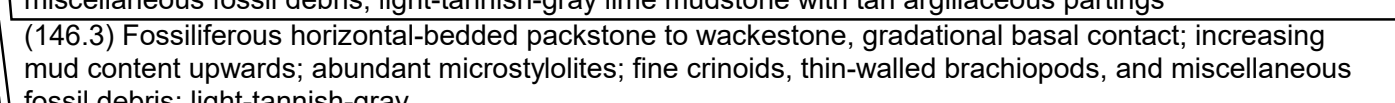

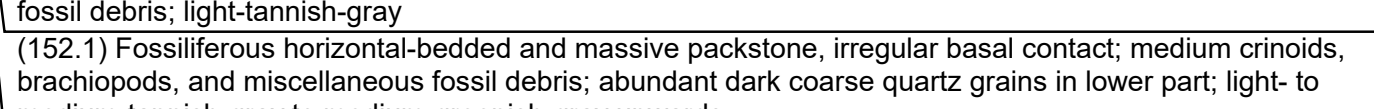

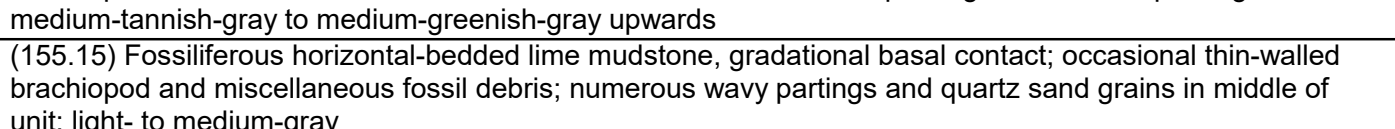

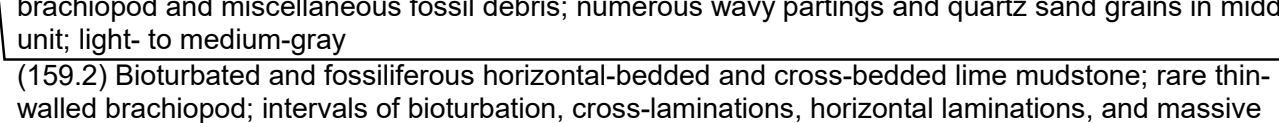
walled brachiopod; intervals of
bedding, light to medium-gray 


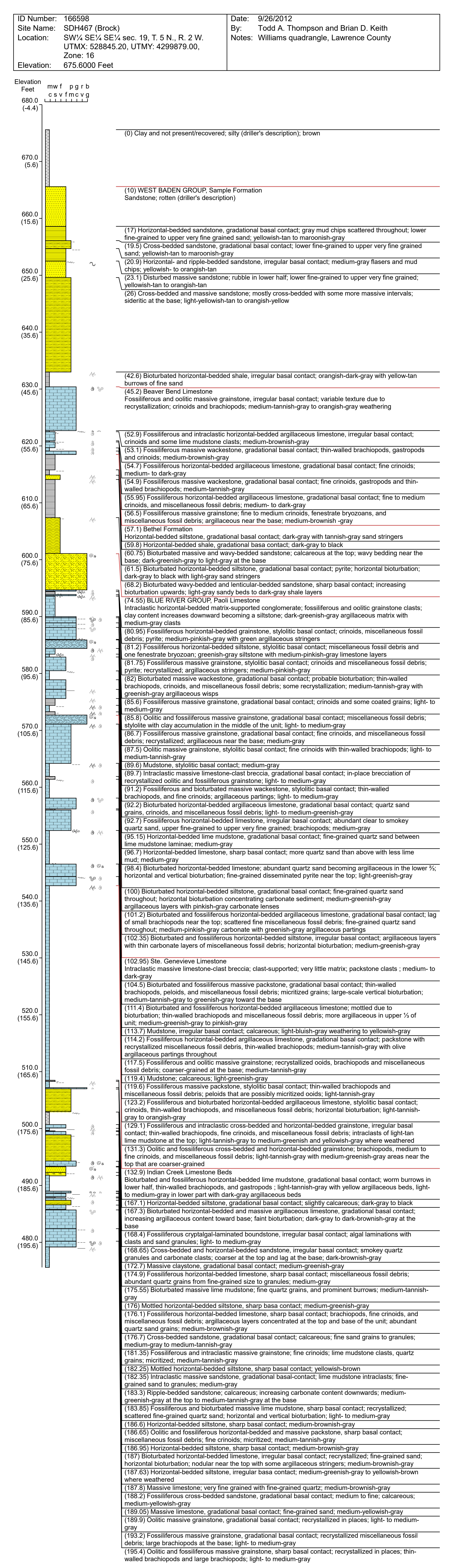




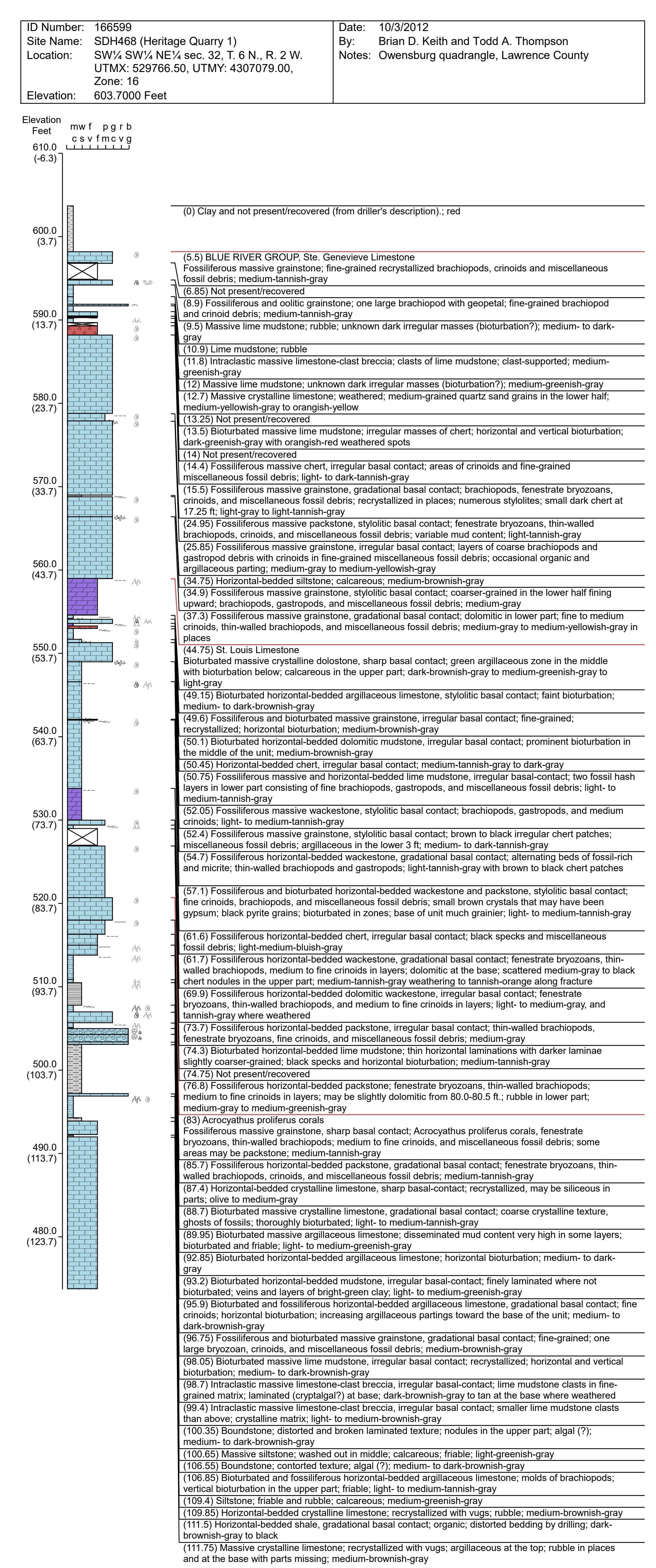




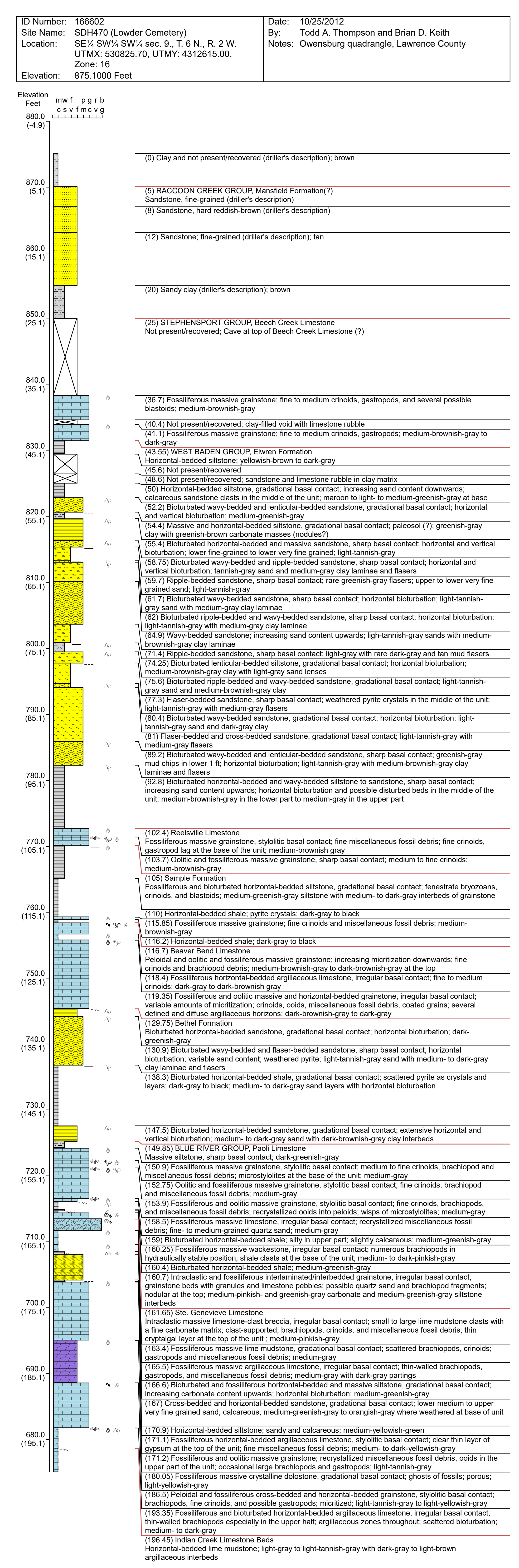




\begin{tabular}{|c|c|c|}
\hline $\begin{array}{l}\text { Do Number: } \\
\text { Site anam: } \\
\text { Location: } \\
\\
\text { Elevation: }\end{array}$ & 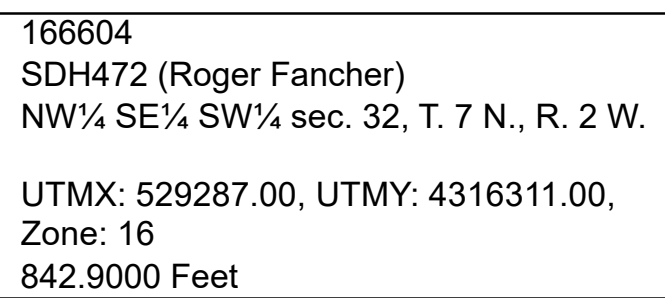 & 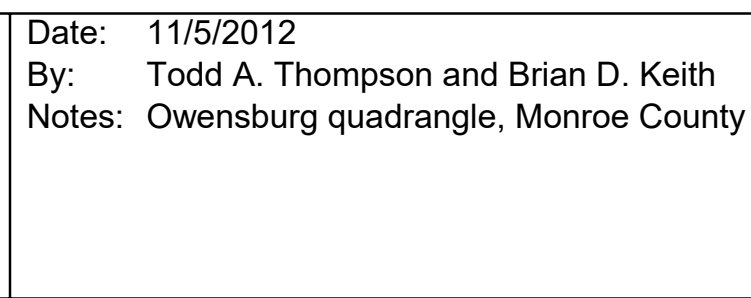 \\
\hline
\end{tabular}

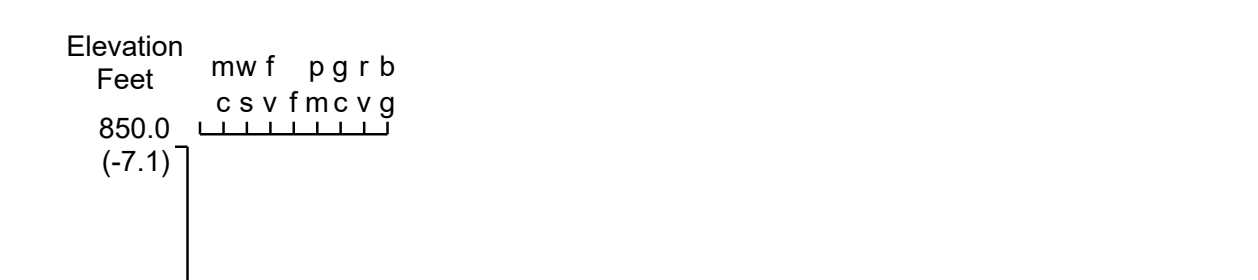

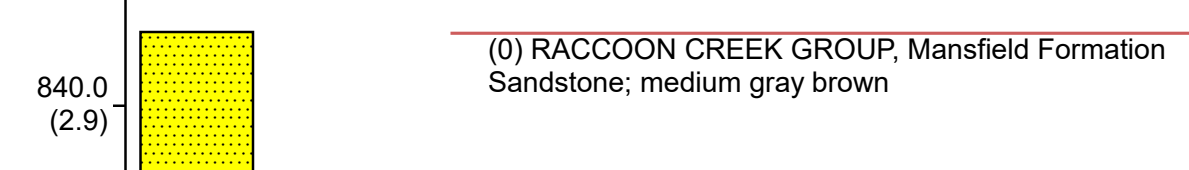

830.0
$(12.9)$

$\overline{(6.5) \text { Sandstone; fine-grained; tan }}$

(18.5) Sandstone: finer-grained than above; darere tan with black streaks

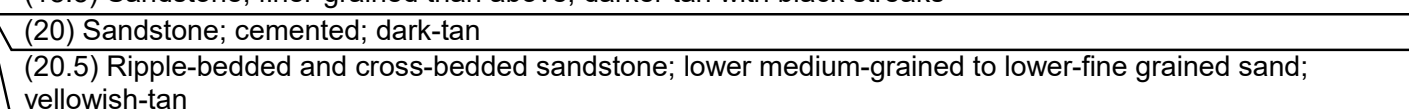

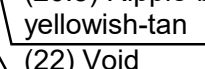

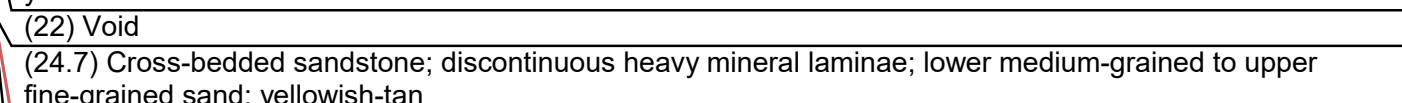

tine-grained sand yellowish-tan
(25.5) STEFHENSPORT GROUP, Becch Creak Limestone

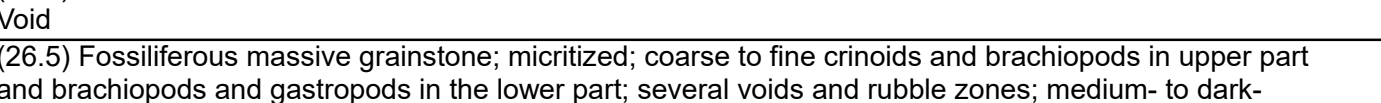

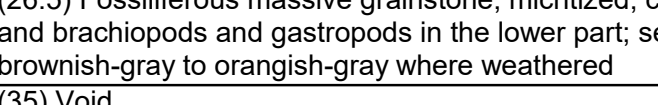

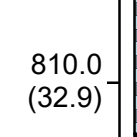

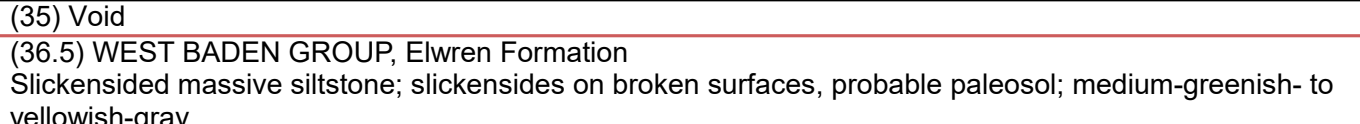

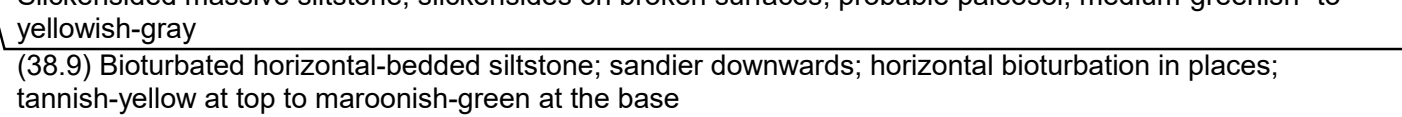
(45.5) Silistone; rubble; maroon

$(48)$ Void

(50.4) Massive mudstone; possible paleosol; maroon to reddish-brown (5). Massive siltstone; irregular carbonate nodules in the lower part; medium-to dark-greenish-gray to
light-greenish-gray

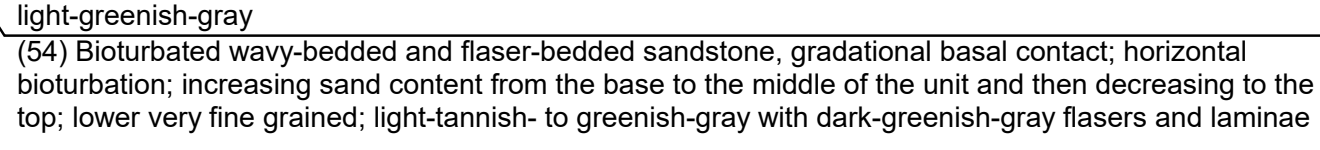

(70.2) Lenticular-bedded and wayy-bedded siltstone, gradational basal contact: wavy bedded near the

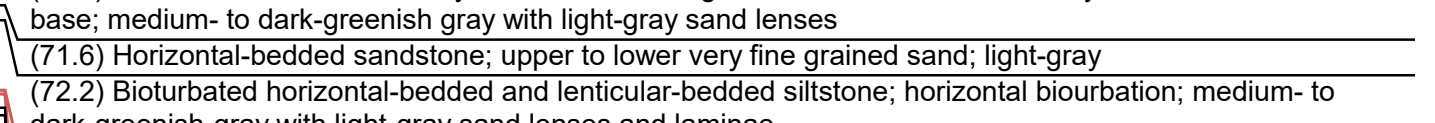

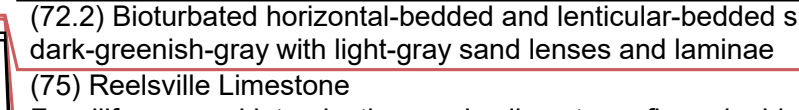

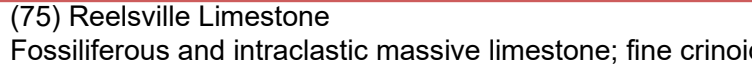

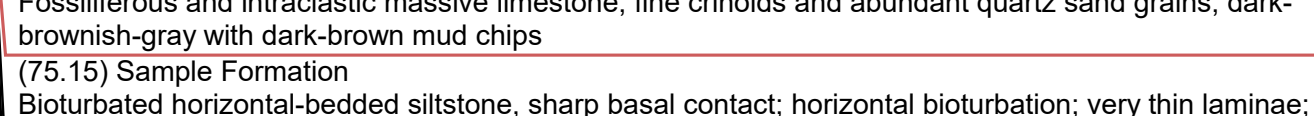
(757) Horizomatay

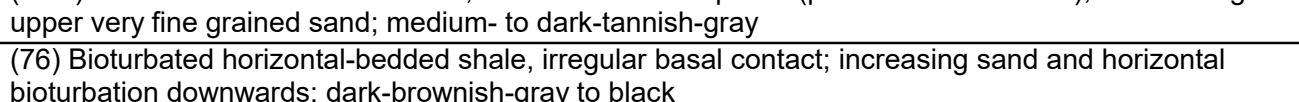

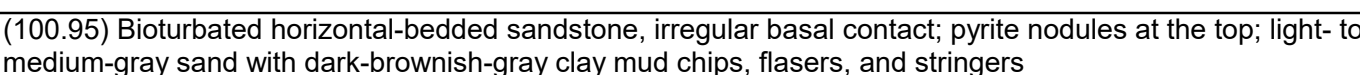

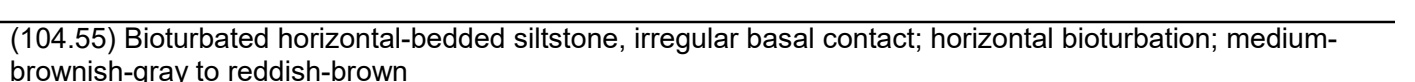

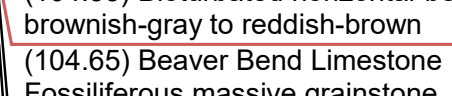

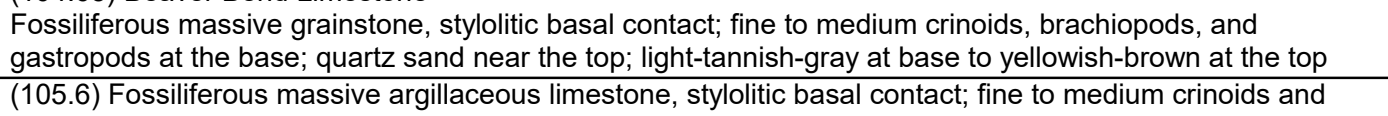

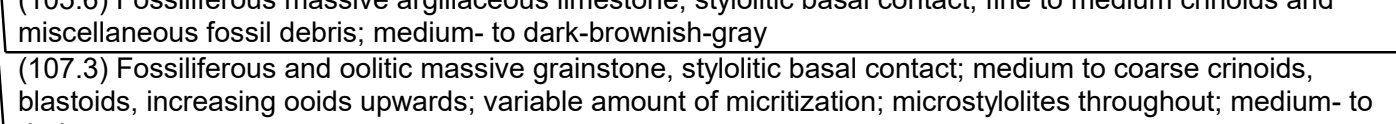

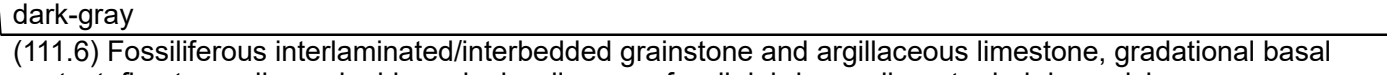

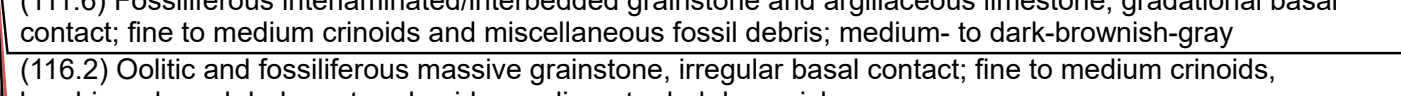

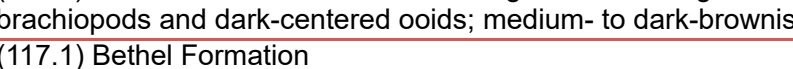

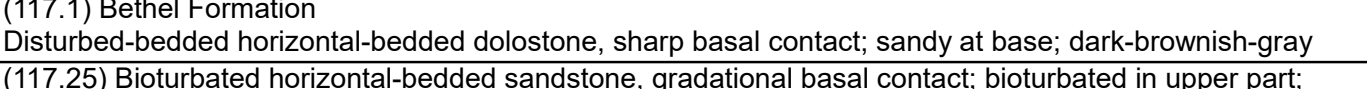

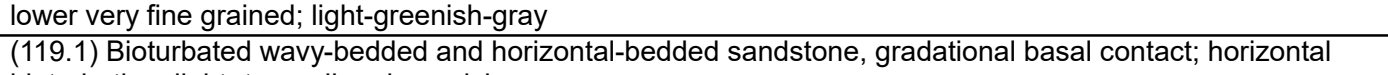

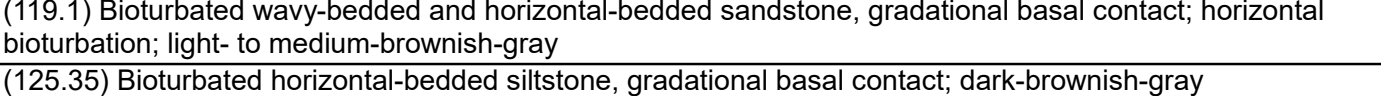

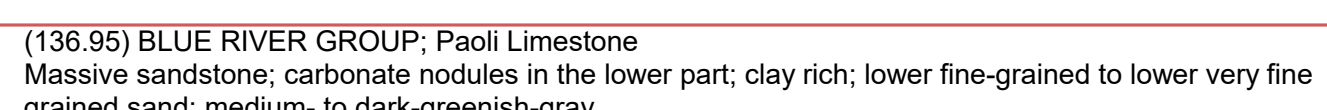

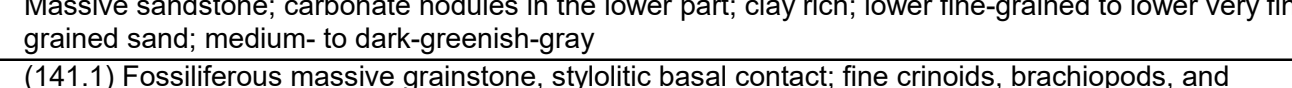

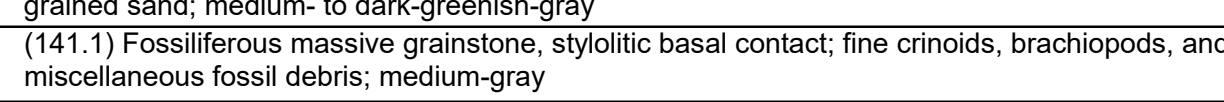

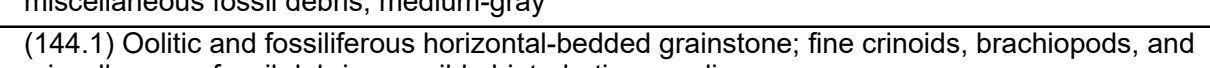

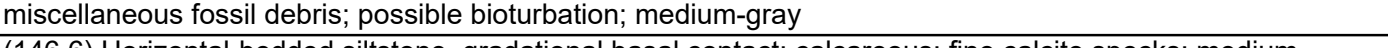

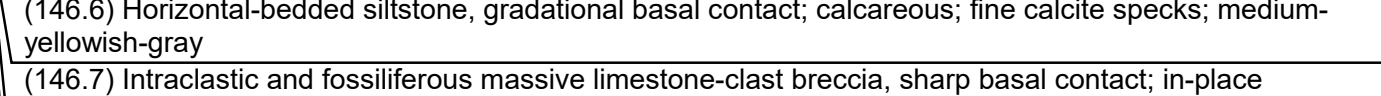

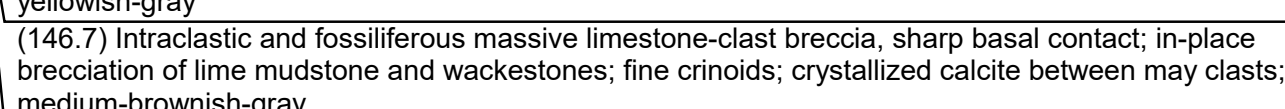

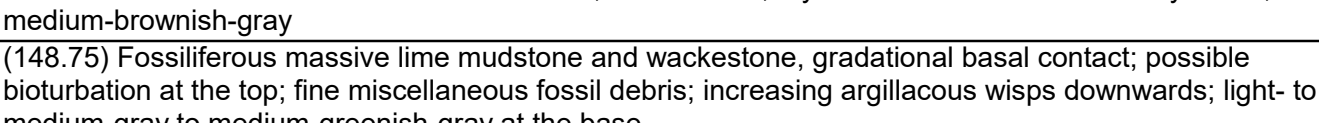

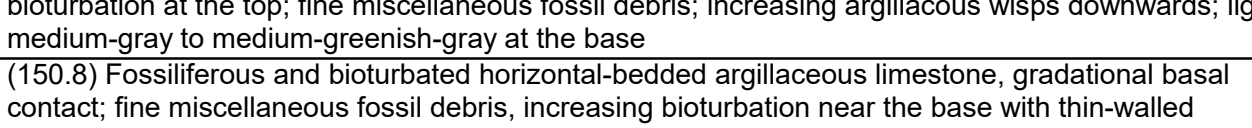

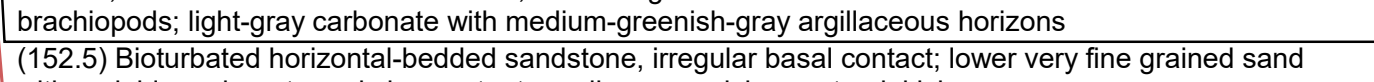

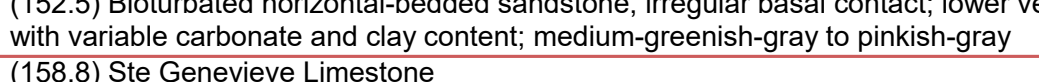

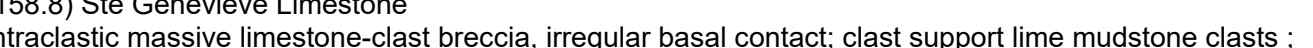

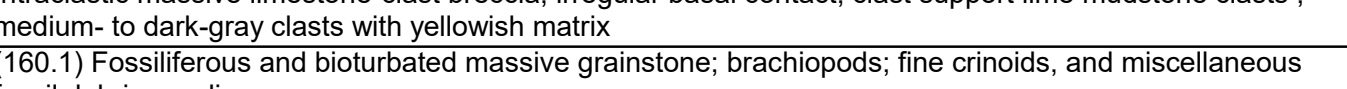

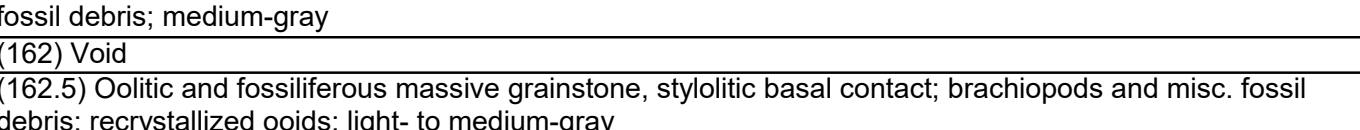

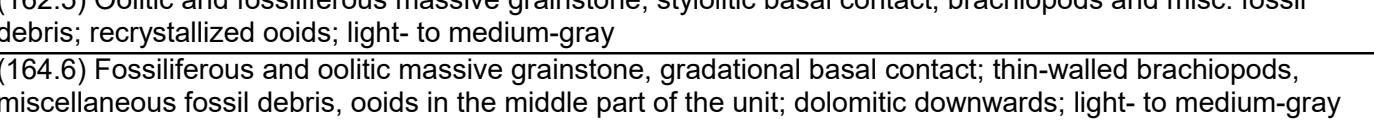

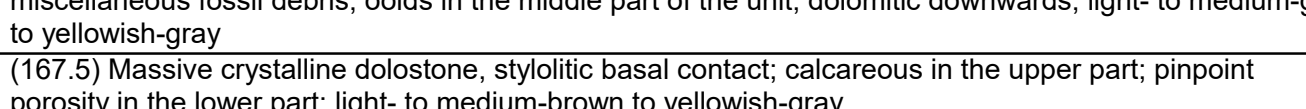

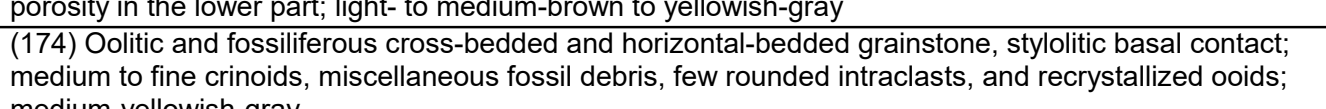

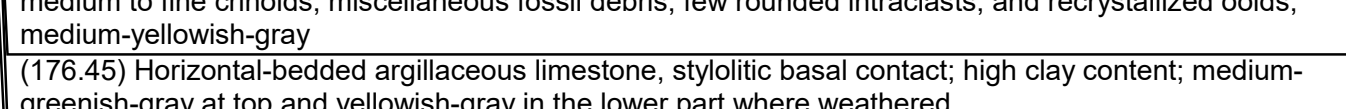

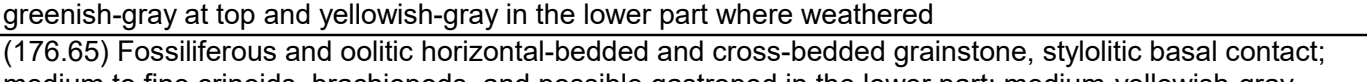

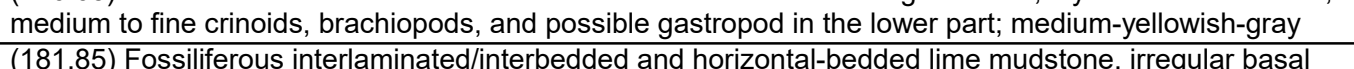

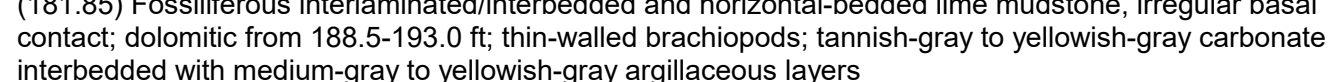
(1973) Massive or 


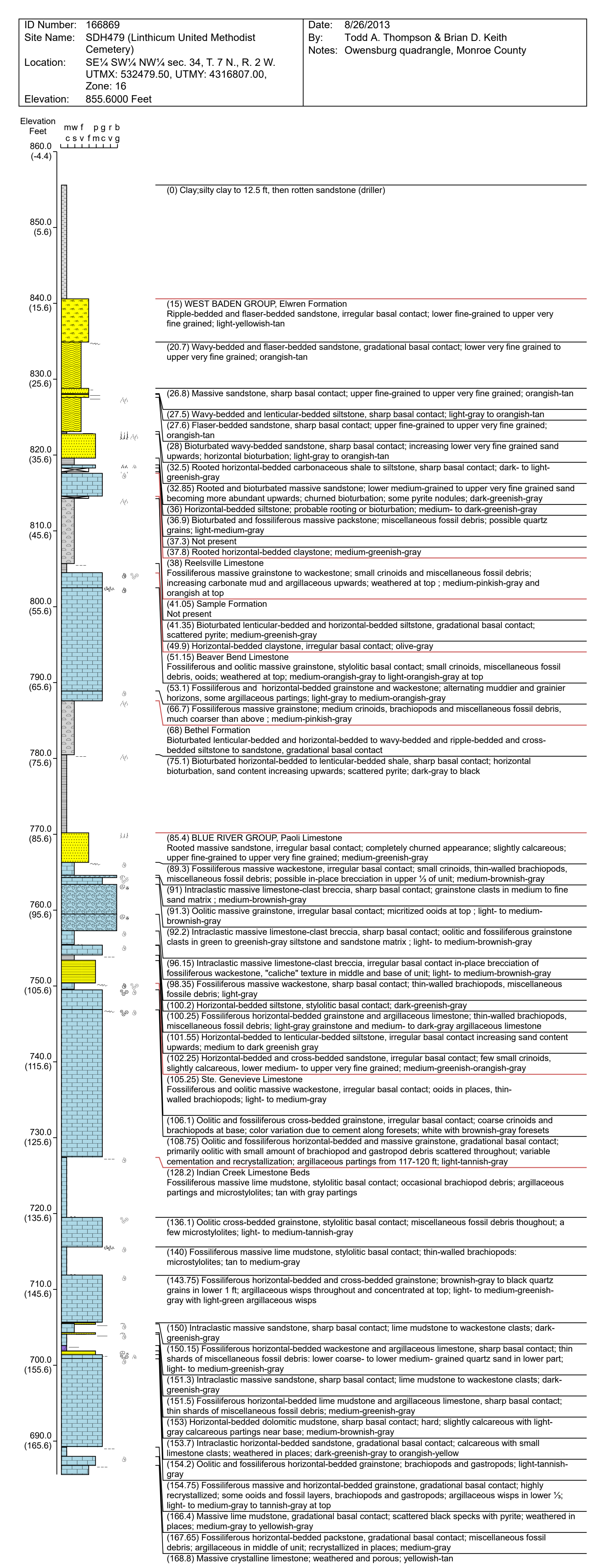


